\title{
INTELLIGENT AGENT CONTROL OF AN \\ UNMANNED AERIAL VEHICLE
}

BY

\section{J. ANDREW CARRYER}

\author{
A THESIS SUBMITTED TO \\ THE FACULTY OF GRADUATE STUdies AND RESEARCH \\ IN PARTIAL FULFILLMENT OF THE REQUIREMENTS FOR THE DEGREE OF \\ MAster of APPliEd SCIENCE
}

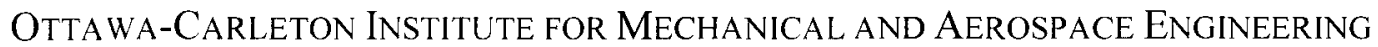

Department of Mechanical and Aerospace EngineEring

CARLETON UNIVERSITY

OtTawa, Ontario

AUGUST 2005

(C) ANDREW CARRYER, 2005 


$\begin{array}{ll}\begin{array}{l}\text { Library and } \\ \text { Archives Canada }\end{array} & \begin{array}{l}\text { Bibliothèque et } \\ \text { Archives Canada }\end{array} \\ \begin{array}{l}\text { Published Heritage } \\ \text { Branch }\end{array} & \begin{array}{l}\text { Direction du } \\ \text { Patrimoine de l'édition }\end{array} \\ \begin{array}{l}\text { 395 Wellington Street } \\ \text { Ottawa ON K1A ON4 }\end{array} & \begin{array}{l}\text { 395, rue Wellington } \\ \text { Ottawa ON K1A ON4 } \\ \text { Canada }\end{array}\end{array}$

Your file Votre référence

ISBN: 0-494-10083-4

Ourfile Notre référence

ISBN: 0-494-10083-4

NOTICE:

The author has granted a nonexclusive license allowing Library and Archives Canada to reproduce, publish, archive, preserve, conserve, communicate to the public by telecommunication or on the Internet, loan, distribute and sell theses worldwide, for commercial or noncommercial purposes, in microform, paper, electronic and/or any other formats.

The author retains copyright ownership and moral rights in this thesis. Neither the thesis nor substantial extracts from it may be printed or otherwise reproduced without the author's permission.
AVIS:

L'auteur a accordé une licence non exclusive permettant à la Bibliothèque et Archives Canada de reproduire, publier, archiver, sauvegarder, conserver, transmettre au public par télécommunication ou par l'Internet, prêter, distribuer et vendre des thèses partout dans le monde, à des fins commerciales ou autres, sur support microforme, papier, électronique et/ou autres formats.

L'auteur conserve la propriété du droit d'auteur et des droits moraux qui protège cette thèse. $\mathrm{Ni}$ la thèse ni des extraits substantiels de celle-ci ne doivent être imprimés ou autrement reproduits sans son autorisation.
In compliance with the Canadian

Privacy Act some supporting forms may have been removed from this thesis.

While these forms may be included in the document page count, their removal does not represent any loss of content from the thesis.
Conformément à la loi canadienne sur la protection de la vie privée, quelques formulaires secondaires ont été enlevés de cette thèse.

Bien que ces formulaires aient inclus dans la pagination, il n'y aura aucun contenu manquant.

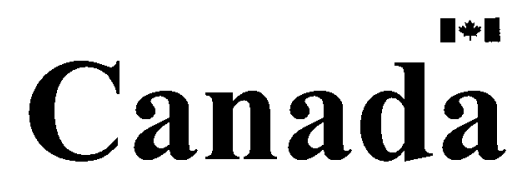




\title{
INTELLIGENT AGENT CONTROL OF AN
}

\author{
UNMANNED AERIAL VEHICLE
}

\author{
SUBMITTED BY \\ J. ANDREW CARRYER \\ IN PARTIAL FULFILLMENT FOR THE DEGREE OF \\ MASTER OF APPLIED SCIENCE
}

Professor P.V. Straznicky

Dr. P. Pace

Thesis Supervisor

Thesis Co-Supervisor

Professor J.C. Beddoes

Chair, Department of Mechanical and Aerospace Engineering

Carleton University

August 2005 


\begin{abstract}
This thesis provides information on intelligent agents and their potential use for controlling an Unmanned Aerial Vehicle (UAV). As the number of aerial vehicles involved in a single mission increases, the current operator-to-vehicle ratio must decrease. With recent advances in automation, artificial intelligence, and especially intelligent agents, it is now possible to make real-time decisions onboard unmanned vehicles. Using the belief, desire, and intention agent model, coupled with data from onboard sensors, a solution is developed in this thesis. Key contributions of this thesis include; the development of a UAV-based Search and Rescue mission concept of operation, the implications of using intelligent agents within a UAV to STANAG 4586, and considerations for the future integration of intelligent agents into a physical UAV system and simulation environment.
\end{abstract}




\section{ACKNOWLEDGEMENTS}

I would like to express my sincere and heart-felt gratitude to Prof. Paul Straznicky, for his assistance, project management guidance, and most of all, for the time and advice he has provided while supporting me through this research project and the graduate program. Also, I would like to thank Dr. Paul Pace, Dr. Phil Farrell, and Daniel Thibault from Defence Research and Development Canada, along with Capt. Ray Stockermans from the Department of National Defence, for their valuable suggestions and insight. Thanks are also in order to Liang Dai, who as a computer science co-op student to this research endeavour, was a phenomenal help.

I would like to acknowledge that partial funding for this project was received from the following organizations: the Department of National Defence, CRESTech, and the Natural Sciences and Engineering Research Council (NSERC). Again, I would like to thank Prof. Straznicky for getting me involved as part of the avionics group within the Carleton UAV project. Through this work, I have learned and experienced many new things that have impacted my research interests.

Last and certainly not least I would like to thank my family who have always supported me in all my endeavours. Mom and Dad have been the sounding board for my ideas, the literary critics of my work, and the cheering squad for my accomplishments. Without their often thankless efforts, and long hours of editing, this thesis could not have materialized to the form that it is in today. To both of you, and Allison thank you very much. 


\section{TABLE OF CONTENTS}

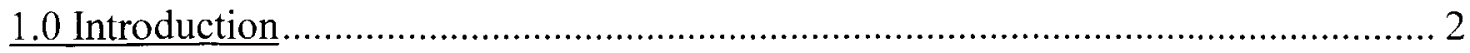

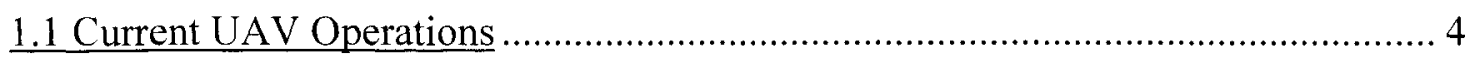

1.2 Global Research and Development Efforts......................................................... 5

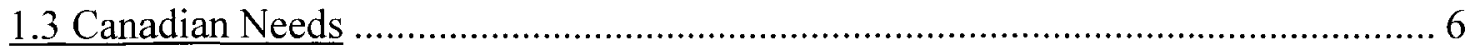

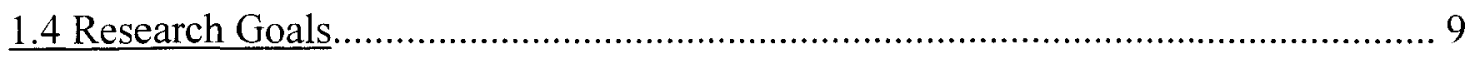

1.5 Thesis Outline

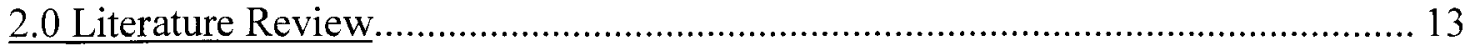

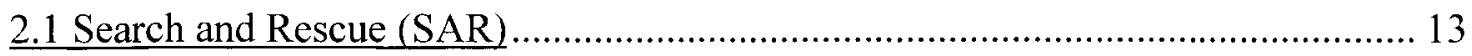

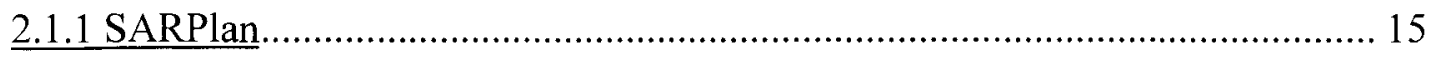

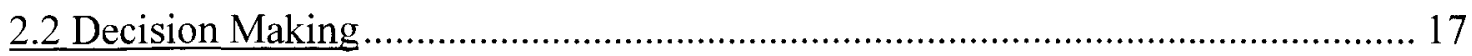

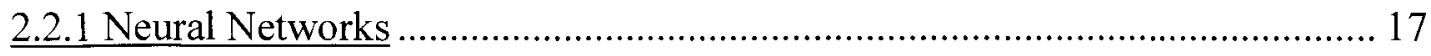

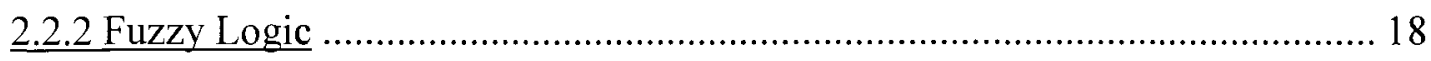

2.2.3 Belief Desire Intention Agents.................................................................... 19

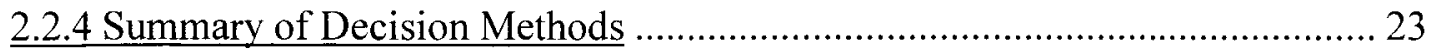

2.3 Agent Oriented Programming......................................................................... 24

2.3.1 Development of Agent Oriented Programming .............................................. 24

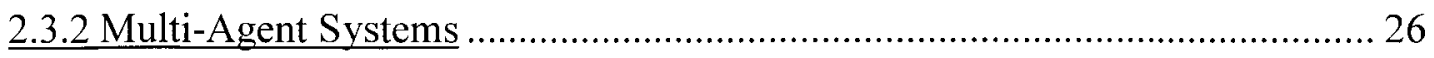

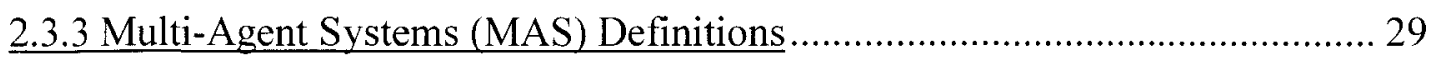

2.3.4 Multi-Agent Systems (MAS) Design Elements.............................................. 30

2.3.5 Multi-Agent Systems (MAS) Design Process ………................................... 32

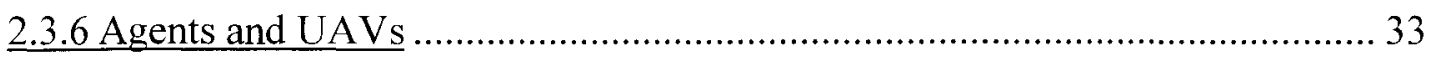

2.3.7 Common Pitfalls of Agent Programming …………….................................... 36 


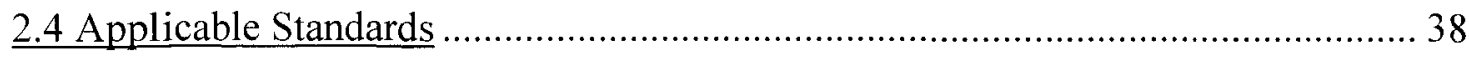

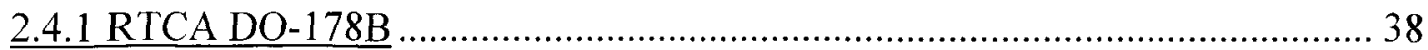

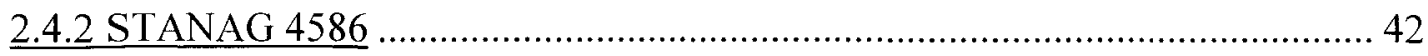

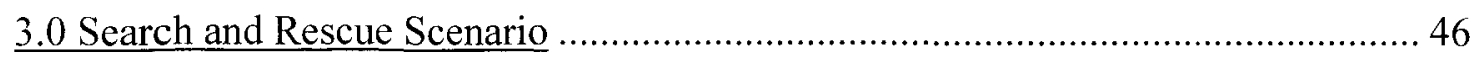

3.1 Overdue Aircraft Scenario .................................................................................... 47

3.2 Intelligence

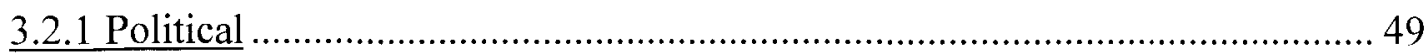

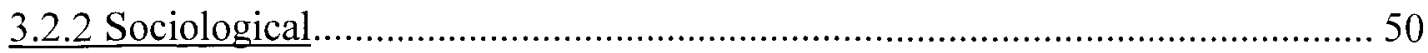

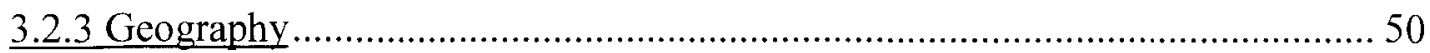

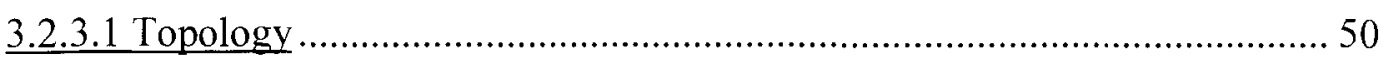

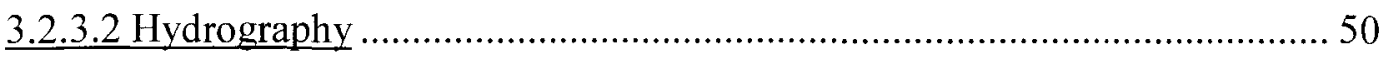

3.2.4 Infrastructure

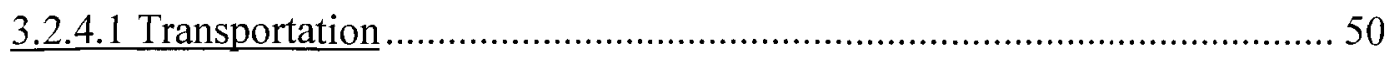

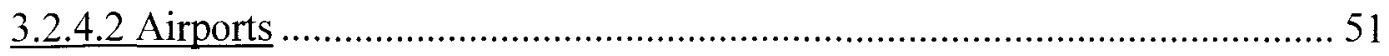

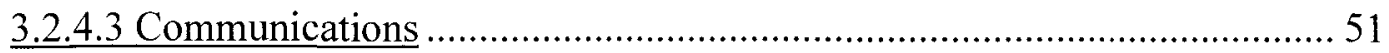

3.3 Miscellaneous Factors..................................................................................... 51

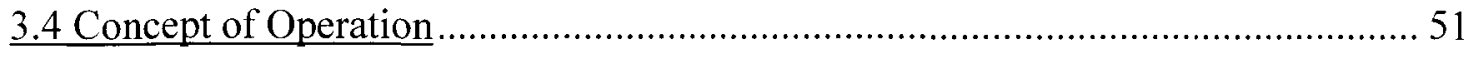

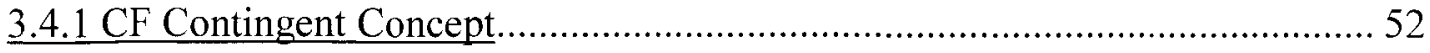

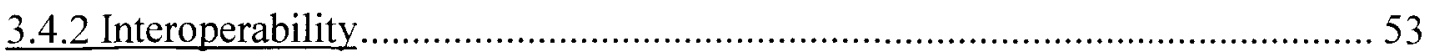

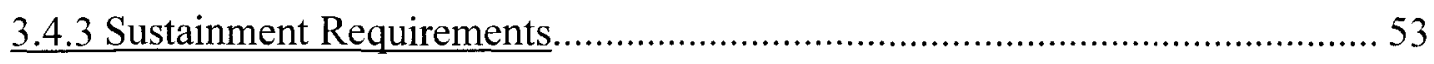

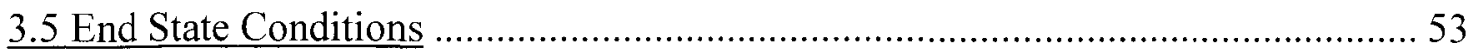

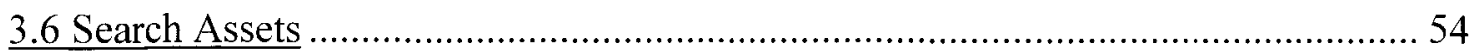

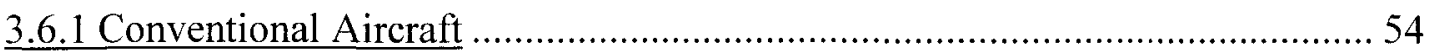




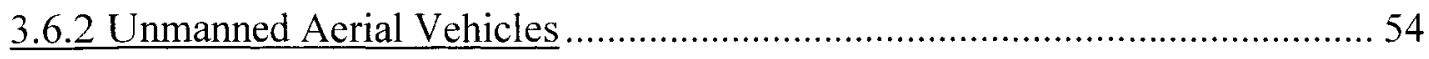

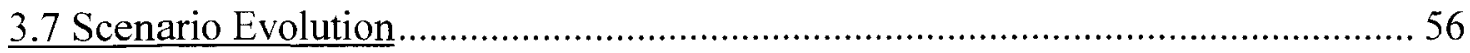

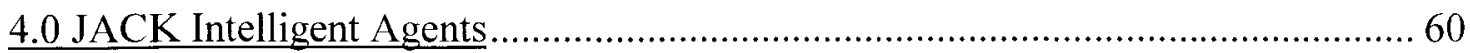

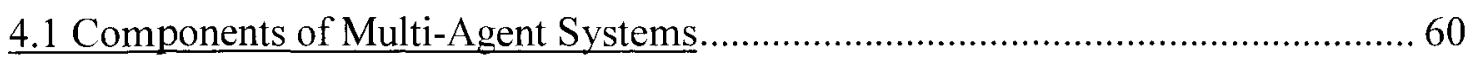

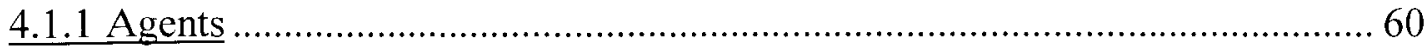

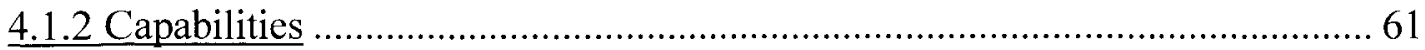

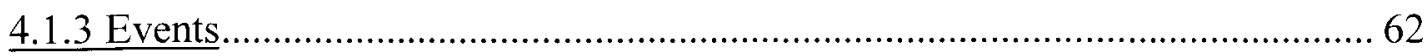

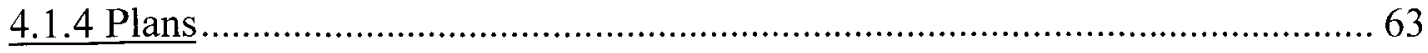

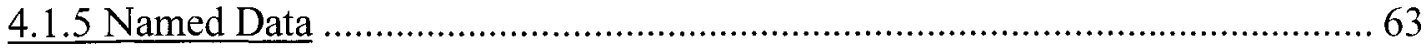

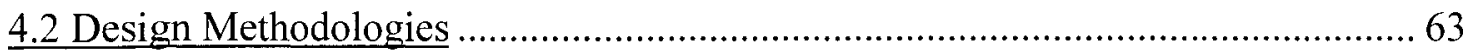

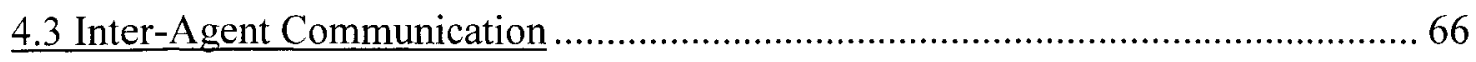

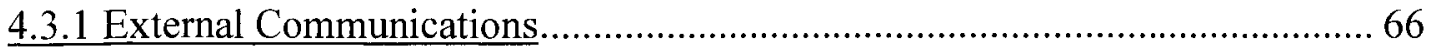

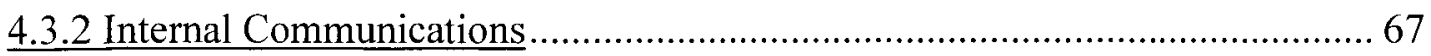

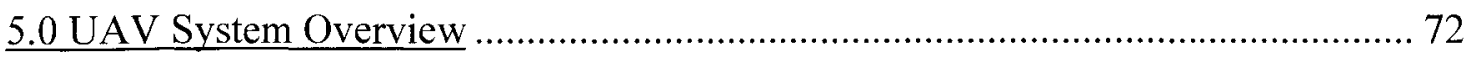

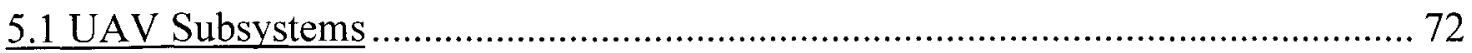

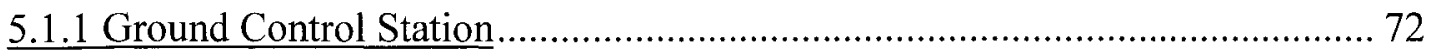

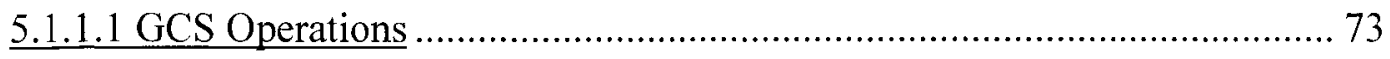

5.1.1.2 Mission Planning Aids and Operator Interfaces ............................... 74

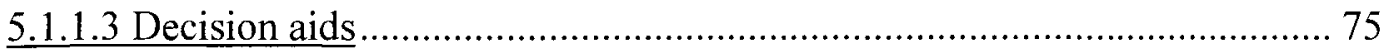

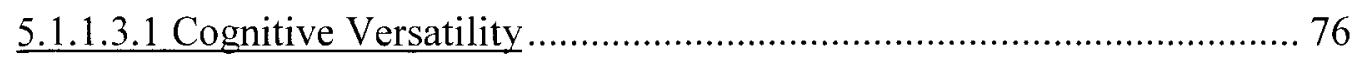

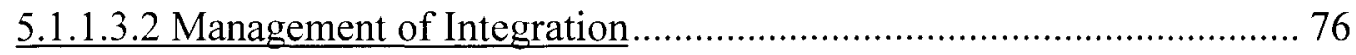

5.1.1.3.3 Management of Complexity ................................................. 76

5.1.1.3.4 Real-Time Performance ...................................................... 77 


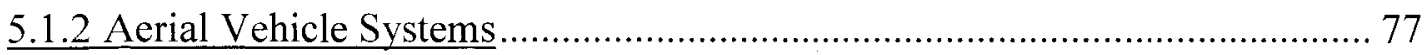

5.1.2.1 Camera

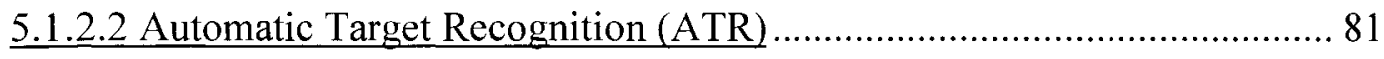

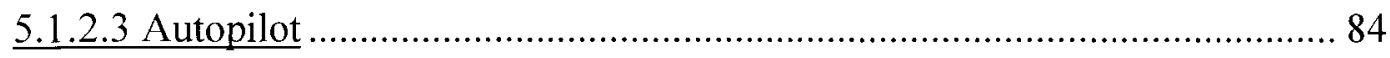

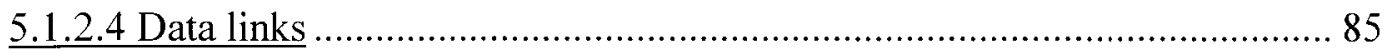

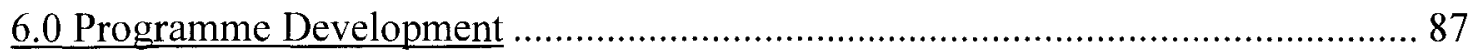

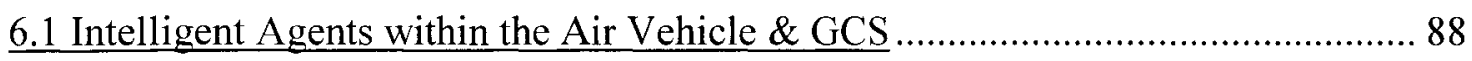

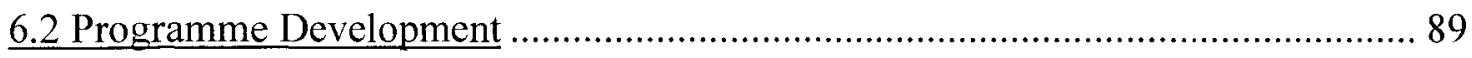

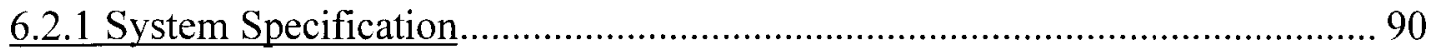

6.2.1.1 Goal Development and Refinement ......................................................... 90

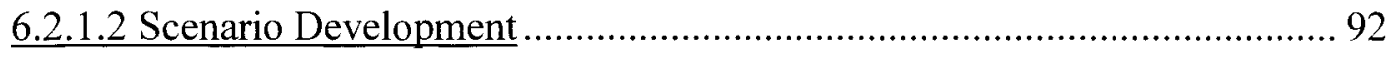

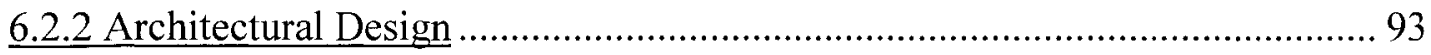

6.2.2.1 Top-Down Approach ............................................................................ 93

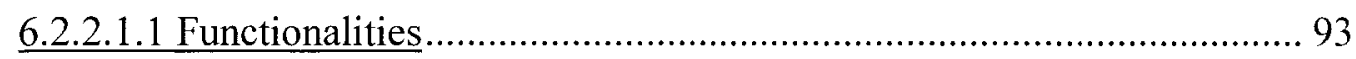

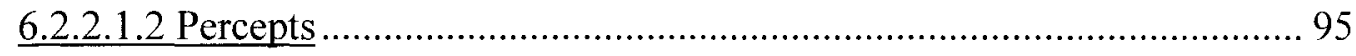

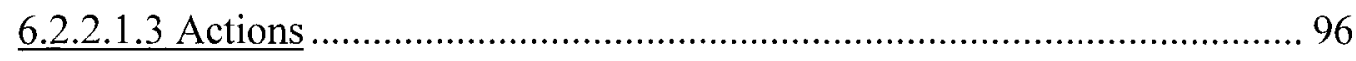

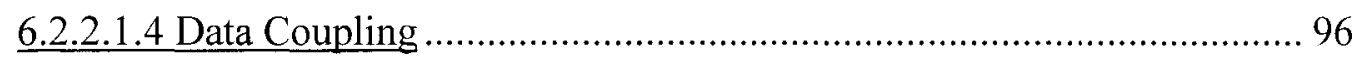

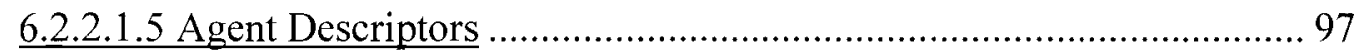

6.2.2.1.6 Interaction Diagrams.................................................................... 100

6.2.2.1.7 Agent System Overview ........................................................... 105

6.2.2.1.7.1 Boundaries of the Agent System............................................. 105

6.2.2.1.7.2 Individual Percept Development............................................... 106

6.2.2.1.7.3 Individual Action Development............................................... 108 
6.2.2.1.7.4 Individual Data Development ...................................... 109

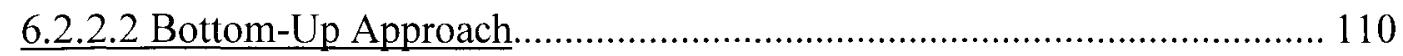

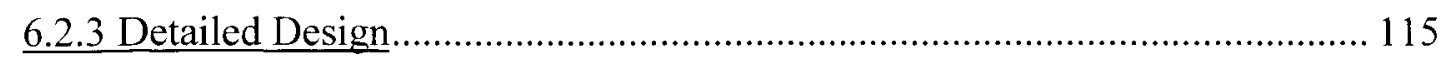

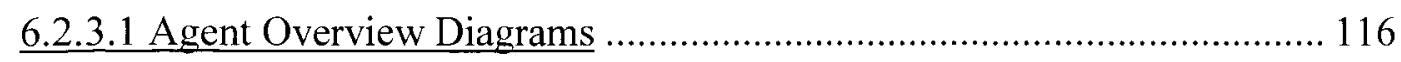

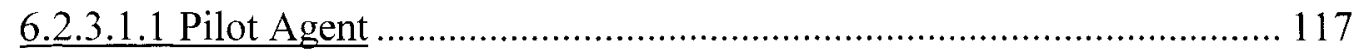

6.2.3.1.2 Feedback Agent .............................................................. 118

6.2.3.1.3 Autopilot Agent ................................................................ 119

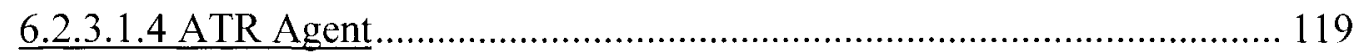

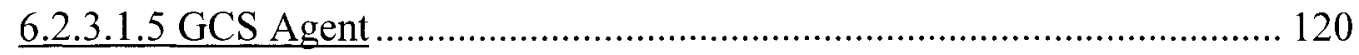

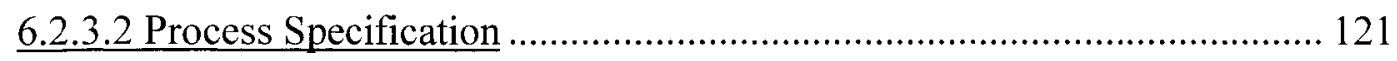

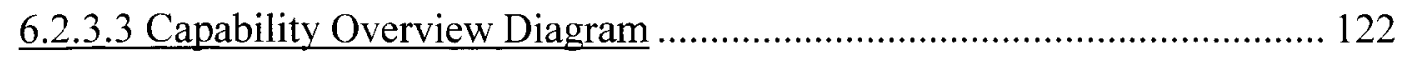

6.2.3.3.1 Maintenance Capability ...................................................... 122

6.2.3.3.2 Closer-Look Capability........................................................ 123

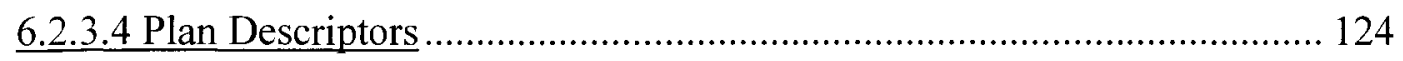

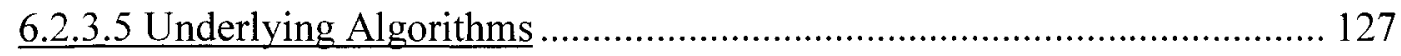

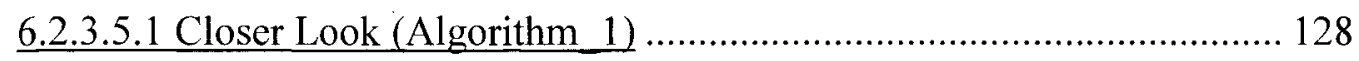

6.2.3.5.1.1 Algorithm 1 mathematics.............................................. 131

6.2.3.5.2 Optimized Path (Algorithm_2) ............................................. 131

6.2.3.5.2.1 Algorithm_2 mathematics......................................... 132

6.2.3.5.3 General Overview (Algorithm_3)...................................... 133

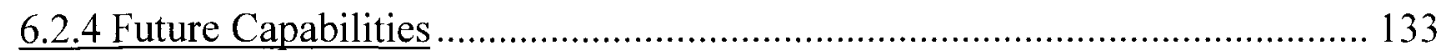

6.2.4.1 Future Implications to $\mathrm{C}^{3}$ Issues ............................................ 134

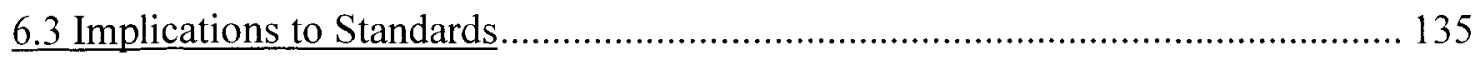


6.4 Summary

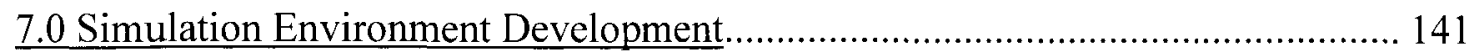

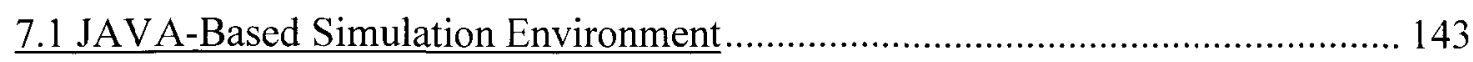

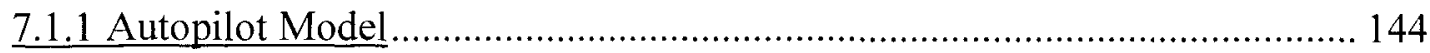

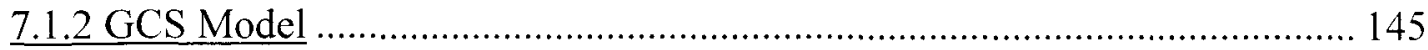

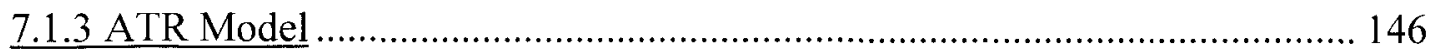

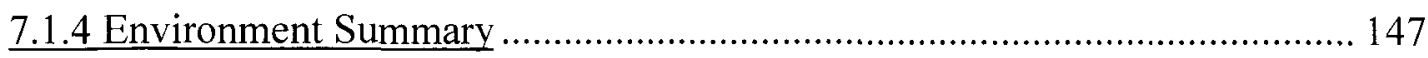

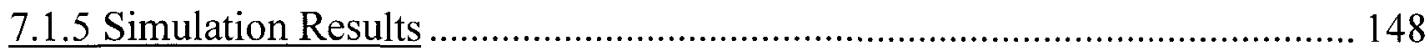

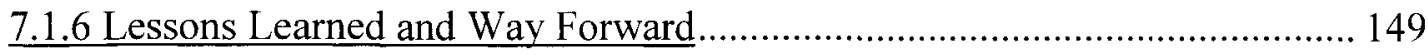

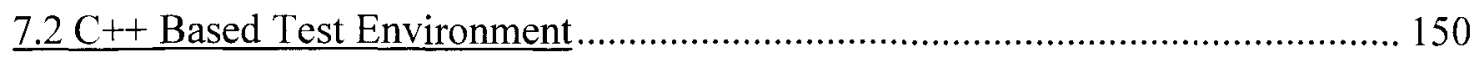

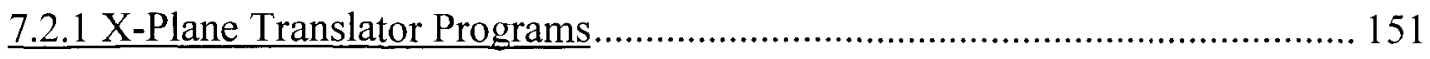

7.2.2 Flight Management Systems Plug-in ........................................................... 153

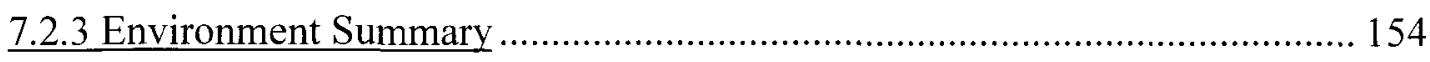

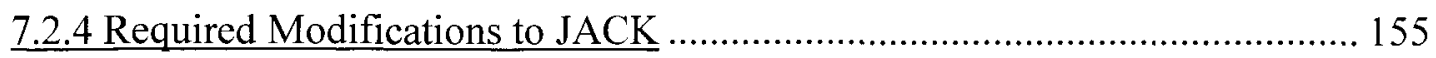

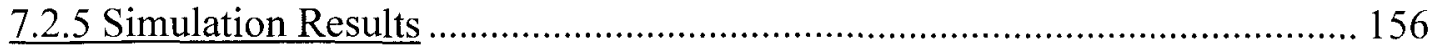

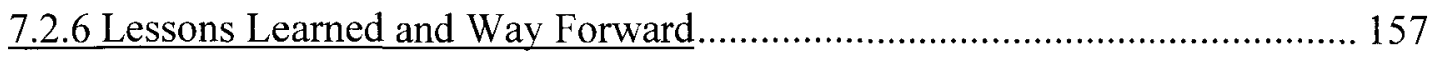

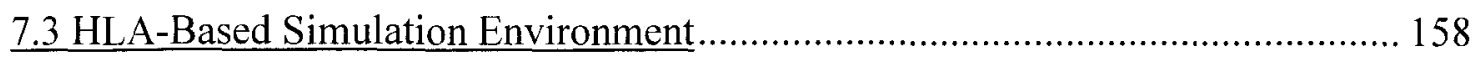

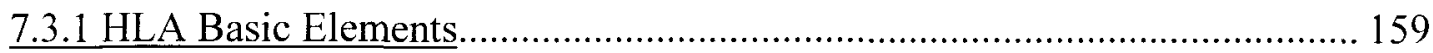

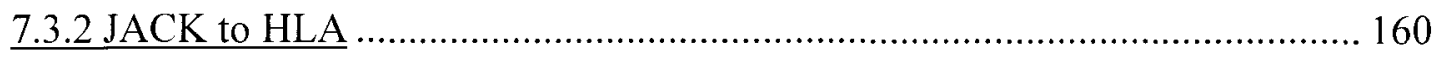

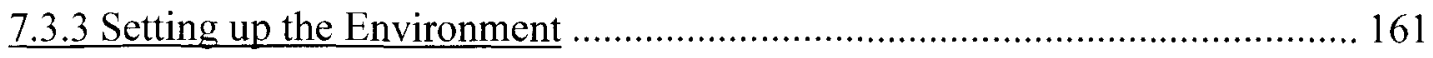

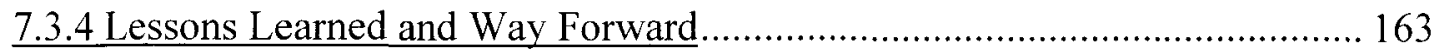

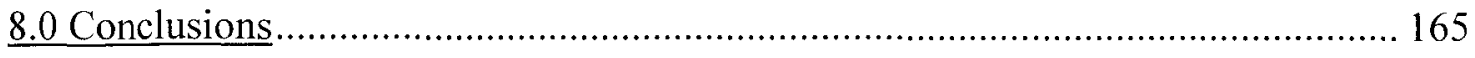

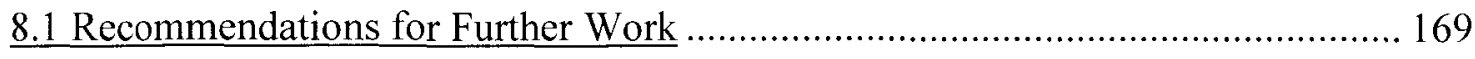


Appendix A: Search and Rescue Practices …………………………......................... 179

A.1 History of SAR Operations in Canada .............................................................. 179

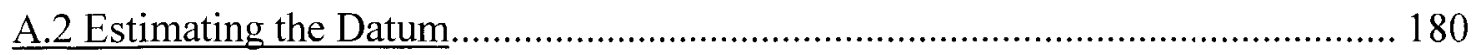

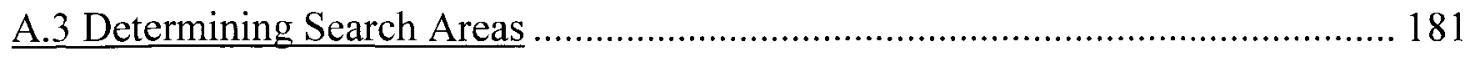

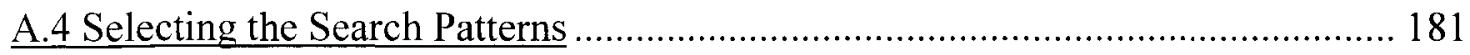

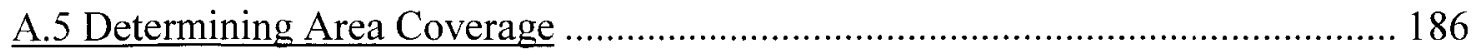

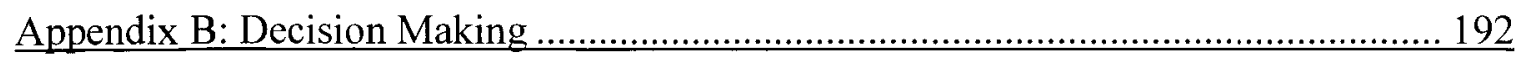

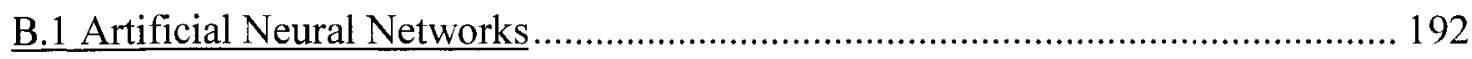

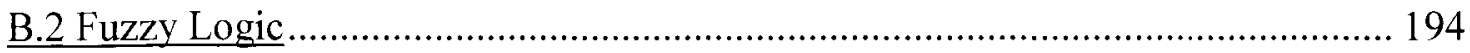

B.3 Human Decision Making .................................................................................. 195

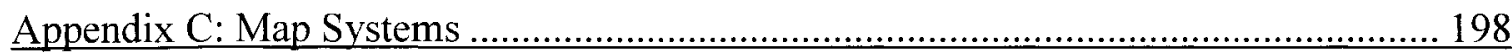




\section{LIST OF TABLES}

Table 1: UAV Categorization by Capability 3

Table 2: The Advantages and Disadvantages of Artificial Neural Networks ___ 18

Table 3: The Advantages and Disadvantages of Fuzzy Logic ___ 19

Table 4: The Advantages and Disadvantages of Intelligent Agents ___ 23

Table 5: GCS Agent Summary ___ 99

Table 6: ATR Agent Summary ___ 99

Table 7: Pilot Agent Summary ___ 99

Table 8: Feedback Agent Summary

Table 9: Autopilot Agent Summary ___ 100

Table 10: Pilot Agent Requirements ___ 111

Table 11: Feedback Monitoring Agent Requirements ___ 113

Table 12: Autopilot Agent Requirements

Table 13: Automatic Target Recognition Agent Requirements ___ 114

Table 14: Ground Control Station Agent Requirements

Table 15: Plan Descriptions ___ _ 124

Table 16: Algorithms for Various Target Confidence Values ___ 128

Table 17: X-plane Data Communications ___ 152

Table 18: X-Plane Index Formats ___ 153

Table C-1: Conversions Tables from Degrees to Metres $\quad 200$ 


\section{LIST OF FIGURES}

Figure 2-1: OODA loop model for coordinator tasks 14

Figure 2-2: Data flow in SARPlan 16

Figure 2-3: Integration of SARPlan into larger UAV-based SAR project 16

Figure 2-4: Developments in autonomy 23

Figure 2-5: Cooperation typology 28

Figure 2-6: Five dimensions of an organization (Ferber, 1999) 31

Figure 2-7: Vehicle control hierarchy using agents 34

Figure 2-8: Role of the VSM 43

Figure 2-9: High-Level Depiction of DLI Interface Content 44

Figure 3-1: Aircraft accidents locations in Canada 46

Figure 3-2: Missing hunting party - area of operation 49

Figure 3-3: Mission coordination through GCS and cueing between UAV platforms _ 58

Figure 4-1: Prometheus methodology overview 65

Figure 4-2: Principal tasks distribution modes 67

Figure 5-1: General ground control station overview (Carryer, 2004) 73

Figure 5-2: Air Vehicle configuration (Carryer, 2004) 77

Figure 5-3: Target geo-positioning through coordinate frame translations 80

Figure 5-4: POD v. POF curve will be sensor based 83

Figure 6-1: The systems engineering process 87

Figure 6-2: Percepts, commands and data flows within the UAV 88

Figure 6-3: Prometheus model for multi-agent programme development 90

Figure 6-4: Goal development 91 
Figure 6-5: Coupled functionalities within the UAV System 94

Figure 6-6: Functionality grouping with data sources 97

Figure 6-7: Agent acquaintance diagram 98

Figure 6-8: Scenario 1 interaction diagram 102

Figure 6-9: Scenario 2 interaction diagram 103

Figure 6-10: Scenario 3 interaction diagram 104

Figure 6-11: Software boundaries with external systems 105

Figure 6-12: Pilot agent overview 117

Figure 6-13: Feedback monitoring agent overview 118

Figure 6-14: Autopilot agent overview 119

Figure 6-15: ATR agent overview 119

Figure 6-16: GCS agent overview 120

Figure 6-17: Plan mapping with start and end states 121

Figure 6-18: UAV flight profile following detection of a possible target. 130

Figure 6-19: The closer-look flight plan, as seen from above. 130

Figure 6-20: Heading between points 132

Figure 6-21: High-Level depiction of DLI content (adapted from STANAG 4586) _ 136

Figure 7-1: Server connection confirmation 144

Figure 7-2: GCS simulation window 146

Figure 7-3: JAVA ATR window 147

Figure 7-4: JAVA client/server interface 148

Figure 7-5: Simulation environment hardware configuration 150

Figure 7-6: $\mathrm{C}++$ Based simulation environment 155 
Figure 7-7: Closely spaced waypoints and X-Plane 157

Figure 8-1: Level of autonomy development at Carleton University 170

Figure A-1: CSAD 1 \& CSAD 2 area definitions (NSM, 1998) 181

Figure A-2: Track crawl patterns (NSM, 1998) 182

Figure A-3: Creeping line pattern (NSM, 1998) 183

Figure A-4: Single unit parallel track (NSM, 1998) 183

Figure A-5: Expanding square (NSM. 1998) 184

Figure A-6: Sector search (NSM, 1998) 185

Figure A-7: Contour search (NSM, 1998) 185

Figure A-8: Coverage factor (NSM, 1998) 188

Figure A-9: POD of the visual system under different search conditions 189

Figure B-1: McCulloch-Pitts neuron model 192

Figure B-2: Online, parallel process training 193

Figure B-3: Schematic of the test-operate-text-exit (TOTE) model 196

Figure C-1: Lambert azimuthal projection on the Earth 198

Figure C-2: Length of degree on meridians 199

Figure C-3: Length of degree on parallels 199 


\section{LIST OF ACRONYMS}

AI

ALNOT

AOP

ATC

BDI

BLOS

BRF

$\mathrm{C}^{2}$

$C^{3}$

CASARA

$\mathrm{CCG}$

$\mathrm{CF}$

CFEC

CMCC

CR-UAV

CSAD

DAI

DARPA

DMSO

DND

DRDC
Artificial Intelligence

Alert Notice

Agent Oriented Programming

Air Traffic Control

Belief Desire Intention

Beyond Line of Sight

Belief Revision Function

Command and Control

Command, Control and Communication

Civil Air Search and Rescue Association

Canada Coast Guard

Canadian Forces

Canadian Forces Experimentation Centre

Canadian Mission Control Centre

Close Range - UAV

Canadian Search Area Definition

Distributed Artificial Intelligence

Defence Advanced Research Projects Agency (USA)

Defence Modelling and Simulation Office (USA)

Department of National Defence (CAN)

Defence Research and Development Canada 


$\begin{array}{ll}\text { DSA } & \text { Detect See and Avoid } \\ \text { DSTO } & \text { Defence Science \& Technology Organization (AUS) } \\ \text { ELT } & \text { Emergency Locator Transmitter } \\ \text { EO } & \text { Electro-Optical } \\ \text { ERAST } & \text { Environmental Research Aircraft Sensor Technology } \\ \text { ETA } & \text { Estimated Time of Arrival } \\ \text { FFSE } & \text { Future Forces Synthetic Environment } \\ \text { FIC } & \text { Flight Information Centre } \\ \text { FOM } & \text { Federation Object Model } \\ \text { GCS } & \text { Ground Control Station } \\ \text { GPS } & \text { Global Positioning System } \\ \text { HALE } & \text { High Altitude Long Endurance } \\ \text { HLA } & \text { High Level Architecture } \\ \text { INREQ } & \text { Information Request } \\ \text { IR } & \text { Infra-red } \\ \text { JRCC } & \text { Joint Rescue Coordination Centre } \\ \text { LKP } & \text { Last Known Position } \\ \text { LOS } & \text { Line of Sight } \\ \text { LRC } & \text { Lateral Range Curve } \\ \text { MALE } & \text { Medium Altitude Long Endurance } \\ \text { MR-UAV } & \text { Missing Aircraft Notice } \\ \text { MANOT } & \text { Multi-Agent System } \\ \text { Mange - UAV }\end{array}$




$\begin{array}{ll}\mu-U A V & \text { Micro UAV } \\ \text { NSM } & \text { National Search and Rescue Manual } \\ \text { OODA } & \text { Orient, Observe, Decide and Act } \\ \text { OOP } & \text { Object Oriented Programming } \\ \text { POC } & \text { Probability of Containment } \\ \text { POD } & \text { Probability of Detection } \\ \text { R\&D } & \text { Research and Development } \\ \text { RCMP } & \text { Royal Canadian Mounted Police } \\ \text { RPV } & \text { Remotely Piloted Vehicle } \\ \text { RTI } & \text { Run Time Infrastructure } \\ \text { SAR } & \text { Search and Rescue } \\ \text { SCPS } & \text { Space Communications Protocol Specification } \\ \text { SRU } & \text { Search and Rescue Unit } \\ \text { SR-UAV } & \text { Short Range - UAV } \\ \text { TCP } & \text { Transmission Control Protocol } \\ \text { UAV } & \text { Unmanned Aerial Vehicle } \\ \text { UCAV } & \text { Unmanned Combat Aerial Vehicle } \\ \text { UDP } & \text { Vehicle Specific Module } \\ \text { VSM } & \end{array}$




\section{Chapter 1: Introduction}

CHAPTER SUMMARY

This chapter provides a general overview of UAV systems, and some of their current uses around the world. Focusing on Canadian needs, and following the results of a 2003 survey of the UAV community in Canada, a number of areas were flagged for further research. Issues listed as high priorities included command control and communications, operations, and increasing vehicle autonomy. Therefore, while focusing on issues relating to autonomy, the overall aim of this thesis is to develop and demonstrate an increase in the decision making authority of UAVs. The objective of this research is to decrease human operator and image analyst workloads and reduce communication bandwidth requirements by increasing the overall autonomy of a UAV system. 


\subsection{Introduction}

This section provides a general introduction to unmanned aerial vehicles within a Canadian and global context, outlines the research goals and provides an overview of the thesis.

Unmanned aerial vehicles (UAVs) exist in many different shapes and sizes to perform a plethora of applications. Over time, UAV systems have evolved from a heritage of remotely piloted vehicles, target drones, and cruise missile technology. Today's UAVs combine sensor and communication equipment with a number of automated systems to create a leading-edge technology. An introduction to UAV systems written by Fahstrom and Gleason (2002) provides a general overview to UAV mission planning, payloads, and data links, along with launch and recovery techniques, highlighting that today's UAVs are truly a system of systems.

Vehicle wingspans range from those fitting in the palm of your hand, up to the size of a Boeing 737. There are close to 500 manufactured models of UAVs, approximately $75 \%$ of which were designed for military applications. Short range and medium range UAVs account for approximately $40 \%$ of all models. Table 1 displays a popular categorization of UAVs (Butterworth-Hayes \& van Blyenburgh, 2004). 
Table 1: UAV Categorization by Capability

\begin{tabular}{|l|c|c|c|c|c|}
\hline UAV Category & Acronym & Range (km) & $\begin{array}{c}\text { Flight } \\
\text { Altitude (m) }\end{array}$ & $\begin{array}{c}\text { Endurance } \\
\text { (hours) }\end{array}$ & Mass (kg) \\
\hline Micro & $\mu$ (Micro) & $<10$ & 250 & 1 & $<5$ \\
\hline Close Range & CR & 10 to 30 & 150 to 300 & 2 to 4 & 150 \\
\hline Short Range & SR & 30 to 70 & 3,000 & 3 to 6 & 200 \\
\hline Medium Range & MR & 70 to 200 & 5,000 & 6 to 10 & 1250 \\
\hline $\begin{array}{l}\text { Medium } \\
\text { Altitude Long } \\
\text { Endurance }\end{array}$ & MALE & $>500$ & 14,000 & 24 to 28 & 1,500 \\
\hline $\begin{array}{l}\text { High Altitude } \\
\text { Long } \\
\text { Endurance }\end{array}$ & HALE & $>2,000$ & 20,000 & 24 to 48 & $>12,000$ \\
\hline
\end{tabular}

Today, teams of operators, flight engineers, maintenance crews and communication managers are required to control the larger vehicles. This requirement to have multiple personnel for a single sensor platform is not desirable, and only by reversing the ratio, will UAV systems become cost effective in civilian applications. Large ground crews are required as today's UAV systems often require the payload operator to remotely control the sensors from the ground. Furthermore, many of the vehicles flown today are controlled as remotely piloted vehicles. Following a controlled-flight into terrain incident in Afghanistan, the accident investigation performed by the Canadian Forces (Directorate of Flight Safety, 2004) concluded that problems in human factors and training were contributing factors to the crash. To prevent this type of problem, the addition of onboard intelligence within a UAV system was previously suggested by Clough (2002). 


\subsection{Current UAV Operations}

In today's global landscape, the predominant users of UAV technologies are military powers. Employed recently in the Balkans, Afghanistan and Iraqi conflicts, UAVs are used as aerial sensor platforms and force multipliers, increasing the capabilities of those who are using them. A single tactical UAV is capable of monitoring dangerous areas of interest, while safely keeping the human operators many kilometres away. Larger, high altitude long endurance (HALE) UAVs have been used for pre- and post-strike assessments, augmenting the role of more expensive and, difficult to obtain, satellite images (Roche, 2003).

Today's UAVs are following the same path as manned aviation did in the early days of flying. Hot air balloons were first used for surveillance and were later replaced by fixedwing aircraft. Military planners soon had surveillance aircraft being used as bombers that were later modified for air-to-air combat. Unmanned combat aerial vehicle (UCAV) projects exist today (Predator A - General Atomics; Neuron - Dassault Aviation; X-45 Boeing) and it is only a matter of time before they form an integral part of the modern battle fleet.

Just as the commercial airline and air cargo industries developed as a result of the experience gained in building military aircraft, UAVs are also being used for nonmilitary applications. In Japan, farmers are using unmanned helicopters to spray chemicals on crops (CASA, 2002), while in Australia and the United States, UAVs are being considered for atmospheric and environmental research. One company in Canada 
is currently testing a small UAV for aeromagnetic surveys (EAGE, 2005), with other companies having expressed similar interest (Sander Geophysics, 2004). From forest fire monitoring to mapping gas and power line routes, there are many potential applications for this technology.

In a recent market report by Frost \& Sullivan (as cited in Wong, 2001) the forecasted revenues from unmanned vehicle technology totaled $\$ 40.3$ billion between 1998 and 2008. While this number may seem high, in the US Department of Defense's UAV Roadmap (OSD, 2002), the US government outlined spending of $\$ 10.8$ billion over the same time period, with $\$ 2.1$ billion allocated for the year 2005 . Worldwide defence spending will continue to account for a large portion of the overall market. As civilian users and uses become more prevalent, new sources of funding and new opportunities will mark the opening of this emerging market.

\subsection{Global Research and Development Efforts}

International UAV manufacturers, along with defence department research and development (R\&D) programs, continue to make advancements in unmanned systems. There are many areas of interest in this evolving sector (NATO 1999, 2002), with growing operational needs and many lessons learned from current systems. Today's users of UAV systems are just starting to realize the full wealth of knowledge, potential and flexibility offered by this technology. As with any new technology, the users' expectations are increasing at exponential rates, thus driving the technology even further. With these rising expectations, new designs and modes of operation are being considered. 
Advances in many different areas of technology are required for these next-generation UAV systems. Work in the area of machine vision continues, as this will be one of the key enabling technologies before $\mu$-UAVs will be able to autonomously navigate hallways and explore rooms. Other priorities for small UAVs include miniaturizing payload packages and avionics, and exploring low Reynolds number aerodynamics. For larger UAVs, R\&D efforts are focusing on the electronics of sensor technology and the requirement for lightweight high-fidelity systems. Propulsion continues to be a significant area of interest especially for the development of small diesel engines and high power density, long-life batteries. Advanced construction materials and methods will continue to aim at producing lighter, yet robust, airframes. A major area of global R\&D effort focuses on UCAV projects. This area alone is leading to advances in Command, Control and Communication $\left(\mathrm{C}^{3}\right)$, stealth technology, and electronic warfare. Current concepts of operation under research include swarming UAVs, employing squadrons of both manned and unmanned aircraft together, and coordinating the efforts of unmanned air and ground vehicles. The associated challenges are daunting and will increase as more users explore UAVs as a viable solution to specific problems.

\subsection{Canadian Needs}

Within a Canadian context, a number of key interest areas have been identified (AeroVations Associates, 2003; Stocker, 2003) for the development of UAV systems. Current research priorities focus on five areas, each of which relates to network-centric operations $\& \mathrm{C}^{3}$. These areas include: 
- Data transmission over limited bandwidth

Encryption and compression of data

- Electronic counter-measures

- Data fusion between multiple platforms

- Inter-vehicle wireless communication

- Ground Control Station

- Automation of mission planning

- Human factors for single and multiple vehicle control

- Autonomous Intelligent Systems

- Methods to measure, sense, interpret and classify a robot's environment, including opportunities and threats

- Adaptive learning structures for collective intelligent systems to cooperate on tasks with minimal direction

- Payloads

- Lightweight, high-resolution/accuracy sensors

- Automatic target recognition capabilities for real-time operations

- Miniaturized payloads for integration into smaller UAVs

- Airspace Integration \& Operations

- Operation of UAVs in civil airspace with manned aircraft

- Detect and avoid studies for collision avoidance

Operator training 
In 2003, AeroVations Associates surveyed 39 key personnel, including representatives from 14 Canadian commercial companies, 16 DND offices (CF Senior military officers, CFEC and DRDC Scientific Advisors), 5 other governmental departments, 3 federal agencies and 1 not-for-profit organization. They reported that $58 \%$ of respondents rated autonomous operations as either a high or very high priority. Other high priority areas among respondents included command control and communications $\left(\mathrm{C}^{3}\right)$, and operations. AeroVations defined operations as including several categories, such as detect-see-andavoid (DSA), airspace integration and air traffic control, ground control systems, and automatic decision aids. In the United States, NASA has teamed with industry and other governmental departments through the Access 5 program. NASA has taken a lead role in DSA research by sponsoring test flights using Scaled Composite's Proteus research aircraft carrying Amphitech's OASys Ka-band radar (Johnsen \& Brown, 2003). While the results are promising for medium range UAVs, the OASys system is large and heavy, making it inappropriate for smaller vehicle systems. It is imperative that UAVs and manned aircraft are able to safely detect and avoid collisions if they are to successfully share the skies. In 2006, NASA Dryden will acquire a Predator B UAV to further support the Access 5 project. The vehicle will serve as a test-bed for demonstrating the operational reliability and system redundancies necessary for allowing UAV operations in US national airspace. 


\subsection{Research Goals}

This thesis is focused on autonomous mission management, which is listed as one of the key priority areas within Canada. The thesis is exploratory in nature. While many different programming methods exist, this thesis aims to evaluate the applicability of intelligent agents to future autonomous UAV operations. This thesis also aims to facilitate future developments by constructing a simple agent-based system and simulation environment.

In order to better understand the issues relating to autonomy, a concept of operation was developed early in the thesis. The original scenario involved multiple UAVs working together to find a ground-based radio emitter. In this scenario three UAVs were used to triangulate the position of the emitter to an approximate region of $5 \mathrm{~km}^{2}$. Then a fourth UAV was tasked to search this region of interest, and visually identify the emitter. Throughout the scenario's development, the objective was to remove the mundane lowlevel tasks that are currently completed by an operator, including flight and sensor controls.

While the proposed electronic warfare-based concept of operation is possible, for the purposes of this thesis it was preferable to use a scenario where open-source literature was widely available. Therefore, two follow-on scenarios were explored including combat and non-combat search and rescue (SAR). 
Fundamentally, a UAV searching for and identifying an emitter within a $5 \mathrm{~km}^{2}$ area, and the search and identification of the missing aircraft in the SAR scenario are similar problems. The only significant difference is the cooperativeness of the sought target, while the elements of searching, identifying and photographing possible objects of interest, remain the same. For a combat SAR mission, the search object may be hiding and therefore be difficult to detect. Furthermore, the search object is not likely to be stationary thereby increasing the complexity of the problem. However, in the case of the non-combat SAR mission, the search object wants to be found. Furthermore, by choosing a non-combat SAR mission it is possible to use pre-established rules and procedures that are published in the public domain.

Therefore, this thesis examines the use of one UAV in a non-combat Search and Rescue mission. This concept of operation is developed in Chapter 3 and forms the basis for assumptions that are made in later sections. The work presented aims to address current Canadian UAV R\&D interests where particular attention is paid to $\mathrm{C}^{3}$ issues through the introduction of autonomous intelligent systems.

One goal of this research is to generate a working Search and Rescue concept of operation that utilizes currently available UAVs and other present-day technologies. The Defence Research and Development Canada (DRDC) Future Forces Synthetic Environment (FFSE) laboratory will eventually be used to simulate the proposed solution and test the concept of operation. The ultimate aim is to integrate this research into an air vehicle platform for flight-testing in order to validate the simulation results. With no 
vehicle currently selected, the work presented herein is applicable to any number of different UAV systems, provided they are compatible with the NATO STANAG 4586 Standard Interfaces of UAV control systems for NATO UAV interoperability.

\subsection{Thesis Outline}

This thesis is presented in eight chapters. Chapter 2 begins with a literature review followed by Chapter 3 which introduces the Search and Rescue scenario for a missing aircraft. Chapter 4 presents the building blocks of the intelligent agent software used to control a single UAV. Chapter 5 describes the subsystems within a UAV system, which include the ground control station, autopilot and onboard sensors. Development of the agent-based program is described in Chapter 6, while three simulation environments are presented in Chapter 7. The conclusions and recommendations for future research directions are presented in the final chapter. The work presented herein contributes to the current state of science at DRDC-Ottawa and to the UAV-related research activities currently underway at Carleton University. 


\title{
Chapter 2: Literature
}

\section{Review}

\author{
CHAPTER SUMMARY
}

This literature review chapter is divided to provide a brief overview to Search and Rescue (SAR) practices in Canada. An introduction to decision making methods is provided before discussing Agent Oriented Programming and some of the common pitfalls faced by designers. The final section provides a discussion on the applicable design standards that are later referenced in Chapter 6 of this thesis. 


\subsection{Literature Review}

This literature review is divided in four subsections. Section 2.1 provides background information on Search and Rescue theory and describes current Canadian SAR practices. Section 2.2 explores various methods for decision-making. Section 2.3 further examines the role and function of intelligent agents as one component of decision-making systems, while Section 2.4 explores two standards that are relevant to the addition of intelligent agents within a UAV system.

\subsection{Search and Rescue (SAR)}

Before describing how a UAV's capabilities could be used within a SAR operation in Chapter 3, it is imperative to understand how current SAR missions are developed and managed. Through discussions with the staff at Joint Rescue Coordination Centre (JRCC) Trenton, and through selected reading of search and rescue manuals and technical reports (NSM, 1998; Abi-Zeid \& Lamontagne, 2003; Soza \& Company, 2004; NSS, 2004; NATO, 1979; Hypher, 1979), this section provides a brief overview of current Canadian practices, where further details can be found in Appendix A.

Abi-Zeid and Lamontagne (2003) present a model of JRCC operations as the first phase in their work of developing computer tools and algorithms that will assist the JRCCs to better prosecute cases. For any SAR operation there is a search coordinator who is responsible for overall command, control and communication. Case prosecution for the coordinator requires the continuous collection and exchange of information between all parties involved in the SAR operation. Abi-Zeid and Lamontagne use an Orient, 
Observe, Decide, and Act (OODA) model shown in Figure 2-1, to illustrate the coordinator's tasks.

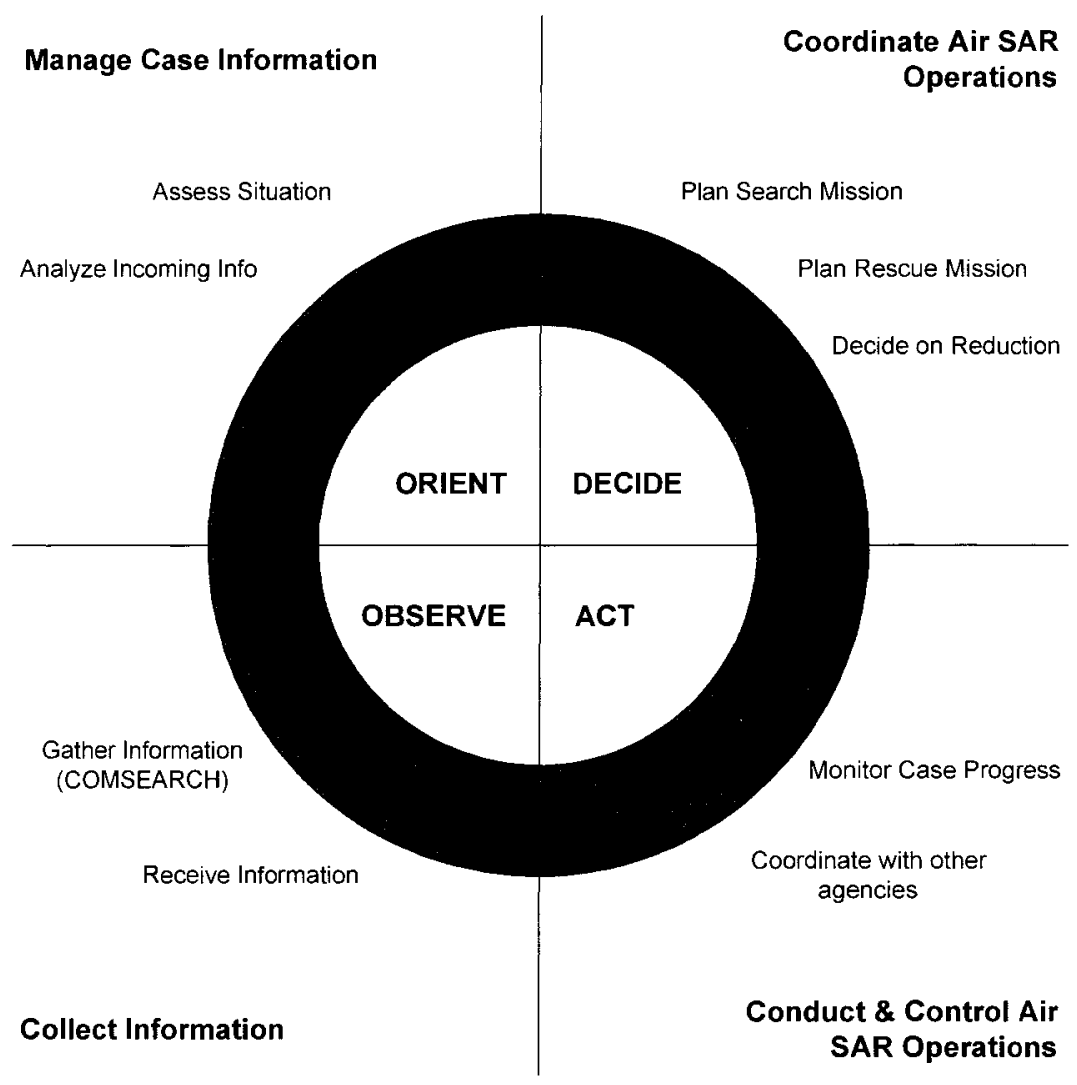

Figure 2-1: OODA loop model for coordinator tasks

After collecting information and assessing the situation, the search coordinator is responsible for planning the search, as defined by the National Search and Rescue Manual (1998). Of key interest to this thesis is the planning of the search. Various search patterns have been developed, and each is further discussed in Appendix A. 


\subsubsection{SARPlan}

This section describes a recent DRDC software design project related to Search and Rescue, SARPlan, and explores how it might be incorporated into a UAV-based SAR mission. Developed by researchers at DRDC-Valcartier, SARPlan is a software tool designed to help search mission coordinators of the Canadian Forces in the optimal planning of overland air search and rescue missions. The goal of this decision support tool is to ensure that the available search assets are deployed in a way that will maximize the mission's probability of success. The core of SARPlan is an optimization module which is based on Koopman's (1980) search theory algorithms, gradient search methods, and on constraint satisfaction programming (Abi-Zeid \& Frost, 2005).

In the initial design of SARPlan the Search and Rescue Unit (SRU) is assumed to be a helicopter, flying at 100 knots. However, the software is flexible and can accommodate other aerial platforms by considering the weight of the search object, the on-scene visibility and the altitude of the SRU. SARPlan can generate the sweep width grid, based on topography and vegetation information for the search area. The results include an effort grid which includes appropriate track spacing for a parallel search pattern, along with the corresponding probability of detection / success / containment, and coverage grids (Abi-Zeid \& Doyon, 2003). The overall flow of information within SARPlan is shown in Figure 2-2 (DRDC-Valcartier, 2002). 


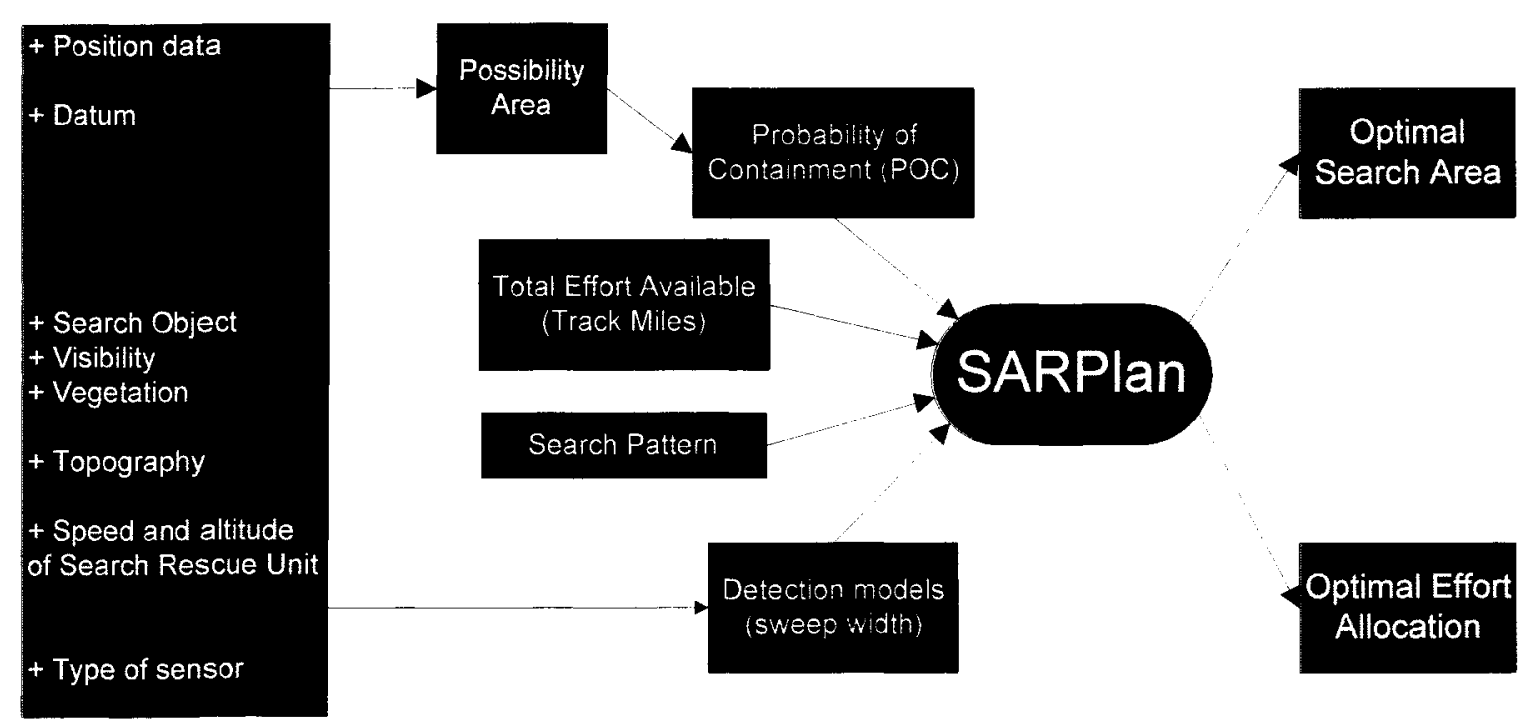

Figure 2-2: Data flow in SARPlan

The optimal search area is one output from SARPlan, and is essentially a set of flight lines, which could be turned into a file of waypoints. Such an extension to SARPlan could then be used as an input to the ground control station (GCS) of an unmanned vehicle. This information could later be sent to the UAV that was conducting the search, as shown in Figure 2-3.

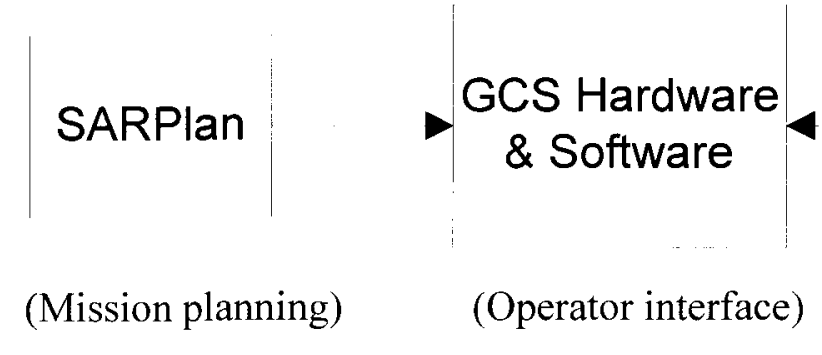

Figure 2-3: Integration of SARPlan into larger UAV-based SAR project

Although the development of SARPlan is complete, additional modifications are possible. The developers' recommendations for future work (Abi-Zeid \& Frost, 2005) include: (1) taking into account any type of SRU, its capabilities, its transit time and the associated 
costs, (2) development of an optimal effort allocation, in the face of uncertainty about the sweep width. These recommendations are consistent with the problems likely to arise while flying a small UAV. Integration of SARPlan into a GCS with subsequent uploading of waypoints to a UAV is beyond the scope of this thesis.

For any SAR operation, the SRU flies a series of search lines which in turn can be related to a series of waypoints. For the purposes of this thesis, the agent-based program must be able to handle this type of information. As such, this concludes the overview of SAR practices and tools that are used in Canada. The following sections of this literature review focus on decision making methods, intelligent agents and design standards.

\subsection{Decision Making}

Advances in automation and artificial intelligence enable real-time decision making onboard an air vehicle. While the focus of this thesis is to develop a solution based on intelligent agents, this section on decision making briefly discusses the development, advantages and disadvantages of two other decision making methods: neural networks, fuzzy logic. Additional discussions are provided in Appendix B for each method. The Belief Desire Intention (BDI) model of human reasoning is also discussed, as this is the reasoning model used by the intelligent agent software.

\subsubsection{Neural Networks}

In 1958, psychologist Frank Rosenblatt invented the first artificial neural network. It was intended to model how the human brain processed visual data and learned to recognize 
objects (Rosenblatt, 1962). Even though neural networks were initially used to model the human brain, it soon became evident that they could be used as a stand-alone tool for solving problems. Because the network finds out how to solve the problem by itself, its operation can be unpredictable and a poorly trained system may produce unexpected results. Furthermore, the structure of a neural network makes it impossible to predict or know the exact flow of information. Table 2 summarizes some of the advantages and disadvantages of neural networks.

Table 2: The Advantages and Disadvantages of Artificial Neural Networks

\begin{tabular}{|l|l|}
\hline \multicolumn{1}{|c|}{ Advantages } & \multicolumn{1}{c|}{ Disadvantages } \\
\hline $\begin{array}{l}\text { No need to know the data relationships a } \\
\text { priori. }\end{array}$ & $\begin{array}{l}\text { Unable to manage imprecise or vague } \\
\text { information }\end{array}$ \\
\hline Capable of self-learning and self-tuning & Unable to resolve conflicts \\
\hline Once trained are extremely powerful & $\begin{array}{l}\text { In optimization problems it is difficult to } \\
\text { reach global minima }\end{array}$ \\
\hline & $\begin{array}{l}\text { Rely on trial-and-error to determine hidden } \\
\text { layers and nodes }\end{array}$ \\
\hline
\end{tabular}

\section{$\underline{2.2 .2 \text { Fuzzy Logic }}$}

In 1965 the concept of fuzzy logic was conceived by Lotfi Zadeh, a professor at the University of California at Berkley, and presented not as a control methodology, but as a way of processing data. Zadeh reasoned that people do not require precise, numerical information input, and are capable of highly adaptive control.

Fuzzy logic incorporates a simple, rule-based ' $I F x A N D$ y $T H E N$ z' approach to solving a control problem rather than attempting to model a system mathematically. The fuzzy logic model is empirically based, relying on experience rather than technical 
understanding of a system. The human brain cannot handle large amounts of isolated, separate ideas at any one time. Instead, the brain tends to bundle similarly related objects into "fuzzy-defined" categories. This categorization assists in processing the information using less complex methods.

Another important feature of fuzzy systems is the ability to define "hedges," which are modifiers of fuzzy values. Modifiers can then be translated to fuzzy statements through mathematical calculations. A summary of this system is provided in Table 3 .

Table 3: The Advantages and Disadvantages of Fuzzy Logic

\begin{tabular}{|l|l|}
\hline \multicolumn{1}{|c|}{ Advantages } & \multicolumn{1}{|c|}{ Disadvantages } \\
\hline $\begin{array}{l}\text { Uses if-then logic rather than complex } \\
\text { differential equations }\end{array}$ & $\begin{array}{l}\text { Cannot handle raw data, including } \\
\text { scientific measurements }\end{array}$ \\
\hline $\begin{array}{l}\text { Is easily understood, without requiring a } \\
\text { controls background }\end{array}$ & $\begin{array}{l}\text { Only able to make decisions based on } \\
\text { subjective information }\end{array}$ \\
\hline $\begin{array}{l}\text { Is often simpler, and therefore less } \\
\text { expensive than other systems for handling } \\
\text { complex information }\end{array}$ & \\
\hline
\end{tabular}

\subsubsection{Belief Desire Intention Agents}

In recent years the Belief Desire and Intention (BDI) model has become one of the bestunderstood and most widely studied models of practical reasoning. The BDI model is largely based on the work of philosopher Bratman (1987) whose work provides a philosophical model of practical reasoning. This BDI model has been used by various militaries in predicting and modelling human behaviour. As part of the human behaviour modelling efforts, intelligent agents were developed. Here each agent uses the BDI model to characterize its beliefs, desires (goals) and intentions. Simply stated, an agent 
will do what it believes will achieve its goals given its beliefs about the world. It is here where we see a major difference between agent and human-based decision making. While agents are able to update their beliefset (understanding of the world around them) they are not able to change their goals.

A BDI agent is also assumed to have a plan library from which it can draw to achieve particular goals, provided a number of preconditions are met. An intention forms when the agent commits to a particular plan or number of steps required to reach its goal. These steps may be sub-goals, and as a result, they may be satisfied by multiple plans. To maintain order, an agent must have a BDI reason engine. This engine is what drives the agent by updating its beliefs, monitoring and updating the goals and intentions, selecting plans to achieve goals, and from this, selecting the actions to perform Norling, 2004). BDI agents use a utility-based decision strategy, where the decisions are made to maximize the utility of the selected course of action (Georgeff et al., 1999). Returning to the basic question of what is an intelligent agent, most researchers in the field of agent systems have agreed that an agent is defined as "a computer system that is situated in some environment, and that is capable of autonomous action in this environment in order to meet its design objectives" (Wooldridge \& Jennings, 1995). This definition is further developed by Ferber (1999), which states that an agent is a physical or virtual entity:

- Which is capable of acting in an environment,

- Which can communicate directly with other agents,

- Which is driven by a set of tendencies (in the form of individual objectives or of the satisfaction/survival function which it tries to optimize), 
- Which possesses resources of its own,

- Which is capable of perceiving its environment (but to a limited extent)

- Which has only a partial representation of this environment (or perhaps none at all),

- Which possess skills and can offer services,

- Which may be able to reproduce itself,

- Whose behaviour tends toward satisfying its objectives, taking into account the resources and skills available to it and depending on its perception, its representations and the communications it receives."

It is widely accepted that to qualify as an agent, a software (virtual) or hardware (physical) system is required to have the following four properties: (1) autonomy, (2) social ability, (3) reactivity and (4) pro-activity.

1. Autonomy implies that the agent has some degree of control over its own actions. Within a fully autonomous system, the agent is not receiving commands from other agents or from a user. Instead, each agent is directed by a set of tendencies or set of individual goals that it is trying to satisfy or optimize.

2. Social ability means the agent is capable of interacting with other agents through some form of language.

3. Reactivity allows an agent, having first perceived and assessed its environment to respond to a changing conditions. Agents are now not only capable of reasoning, but also of acting as is the case in classical artificial intelligence. In acting, they will change their environment, which will affect subsequent decisions. 
4. Pro-activity allows the agent to work with goal-directed actions. The agent's behaviour attempts to satisfy its objectives after taking into account the resources and skills that are required and available. This will depend on its perception and on the communications that the agent receives.

By understanding these definitions the reader can start to appreciate the strength and power that agent programming provides to the practitioner, and the vast areas of potential applications.

Multi-agent systems (MAS) are simply an extension to individual agents, where multiple agents may exist in the same or different environments, offering different skills and services. Furthermore, agents in MAS require the ability to cooperate, coordinate and negotiate with other agents.

When an agent-oriented view of the world is adopted it soon becomes apparent that most problems require or involve multiple agents to represent the decentralized nature of the problem, the multiple loci of control, and the multiple perspectives or the competing interests (Bond and Glasser, 1988). Moreover, Jennings (1999) states that within this new view of the world, agents need to interact with one another, either to achieve their individual objectives or to manage the dependencies that ensue from being situated in a common environment. The net results are multi-agent systems. The advantages and disadvantages of an agent-based system are summarized in Table 4. 
Table 4: The Advantages and Disadvantages of Intelligent Agents

\begin{tabular}{|l|l|}
\hline \multicolumn{1}{|c|}{ Advantages } & \multicolumn{1}{c|}{ Disadvantages } \\
\hline No need for training programs & High program complexity \\
\hline Ideal for reacting in changing environments & Lack of development standards \\
\hline Distribution means faster processing & Possible to have conflicting intentions \\
\hline
\end{tabular}

\subsubsection{Summarv of Decision Methods}

Intelligent agents are unique in that they exhibit similar behaviour to both neural network and fuzzy logic systems in terms of their ability to reason and adapt. Furthermore, by virtue of being distributed, at any one time, all the agents in the multi-agent system are active and capable of making independent decisions. These are two key advantages for choosing to use intelligent agents over neural networks or fuzzy logic for the high-level mission management of an unmanned vehicle. Using these two metrics, a comparison of available methods is provided in Figure 2-4. Clearly, multi-agent systems are a possible solution for operating within a constantly changing environment, where both proactive and reactive decision making is required.

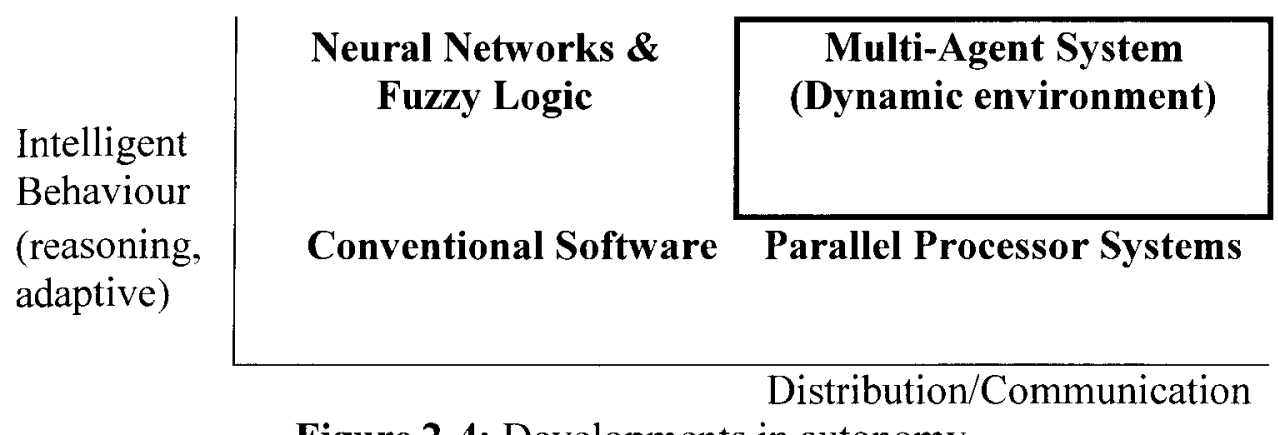

Figure 2-4: Developments in autonomy 


\subsection{Agent Oriented Programming}

Having initially chosen to develop a solution using intelligent agents, this section introduces and describes agent oriented programming (AOP), its development, properties and applicability to controlling UAVs. From decision aids to autonomous decisionmaking, agent oriented programming offers many new possibilities within the gamut of UAV operations.

\subsubsection{Development of Agent Oriented Programming}

As software development progressed through the years from machine code and assembly language through to the advent of sub-routines, procedures and functions, the level of abstraction increased substantially. Today, object oriented programming is at the forefront of many modern software engineering applications. Agent oriented programming is leading to a shift in software development, to a different level of abstraction.

Agent oriented programming is a logical extension of object oriented programming (OOP), and understanding the relationship between the two concepts is important. Under OOP, an object uses methods (behaviours, functions and procedures) that require inputs from the object's public or private attributes (data, state information). Often, public methods and attributes are referred to as the interface of the object, where communication between objects occurs by invoking the public methods of other objects. Both OOP and AOP share the principle of information hiding within private classes, and recognize the importance of interactions. 
Wooldridge (2002) presented three main differences between object and agent-oriented programming:

1. Where it is possible to control an object from the outside (i.e. whitebox control), agents have an autonomous behaviour which cannot be directly controlled from the outside (i.e. blackbox control). Agents, unlike objects, are able to decide for themselves whether or not to perform an action on request from another agent.

2. Agents are smart and capable of reactive, proactive, and social behaviour. Objects are the combination of data structures and methods, often representing passive entities, as opposed to the active entities represented by agents.

3. Agents are active within MAS where an agent may have at least one thread of active control (multi-threaded). Object oriented programming has historically been single-threaded, though new developments have allowed multi-threading applications.

Within agent oriented programming, each agent has a degree of control and decides what actions to perform at what time, based on both external environmental conditions and private beliefs and desires (Tesfatsion, 2004). By comparison, objects are generally passive in nature: they need to be sent a message before they become active. While objects are able to understand state information and behaviour details, they do not encapsulate the ability to act. Unfortunately, object-orientation fails to provide an adequate set of concepts and mechanisms for modelling complex systems. Individual objects represent behaviour at a very fine level of detail, while methods are too primitive 
for describing the interactions that take place. However, agents provide a coarser level of detail and a higher-level view of interactions.

The distinctions start to blur when we compare agents to object-oriented expert systems; however, two key differences remain. Agents are situated in an environment and are aware of their surroundings, and the agents are capable of acting on their own, while objects are not. The difficulty comes when comparing multi-agent systems to expert systems for real-time process control, in which case both systems have similar abilities. Agent oriented programming permits real-time control within a highly flexible environment. The coding itself is straightforward for programmers and follows an intuitive and structured format. Most importantly, agent oriented programming is apt to high-level thinking. Within agent oriented programming (AOP), the developer is able to focus on the high-level goals and on plans for reaching these goals.

This said, agent-based approaches will not supplant object-oriented techniques. Instead agent-based computing provides a higher level of computational abstraction, which may be realized through object-based systems (Jennings 1999). It is acknowledged that advances in object-oriented programming may be able to yield similar results to those presented in this thesis using multi-agent systems.

\subsubsection{Multi-Agent Svstems}

In the last decade, multi-agent systems (MAS) have emerged as a field of great interest to the computer science and artificial intelligence (AI) communities. MAS introduce new 
tools and concepts that are being used to tackle both existing AI problems and focus on new challenges within mobile robotics and networked systems. MAS have evolved from two areas of research: distributed artificial intelligence (DAI), and artificial life. DAI was widely developed in the 1980s at a time when the Defense Advanced Research Projects Agency of the United States was heavily funding AI activities. From DAI comes the concept of cognitive agents whose origin lies in the communication and cooperation associated with classical DAI expert systems. The key contributions to the field of artificial life have come within the last ten to fifteen years. Artificial life puts an emphasis on behaviour, autonomy and viability for making reactive agents. A reactive agent is one that acts only following a trigger and remains dormant the remainder of the time.

Multi-agent systems also introduce the concepts of collective intelligence and emergent cooperative behaviour, concepts that were not found in earlier AI theories. With emergent behaviour examined from an observer's viewpoint, the agents appear to be working together, but from the agent's viewpoint they are not. Instead, the agents are simply carrying out their own individual behaviour (Doran et al., 1997). Figure 2-5 presents a classification for the various types of cooperation within a multi-agent system. 
Multi-Agent Systems

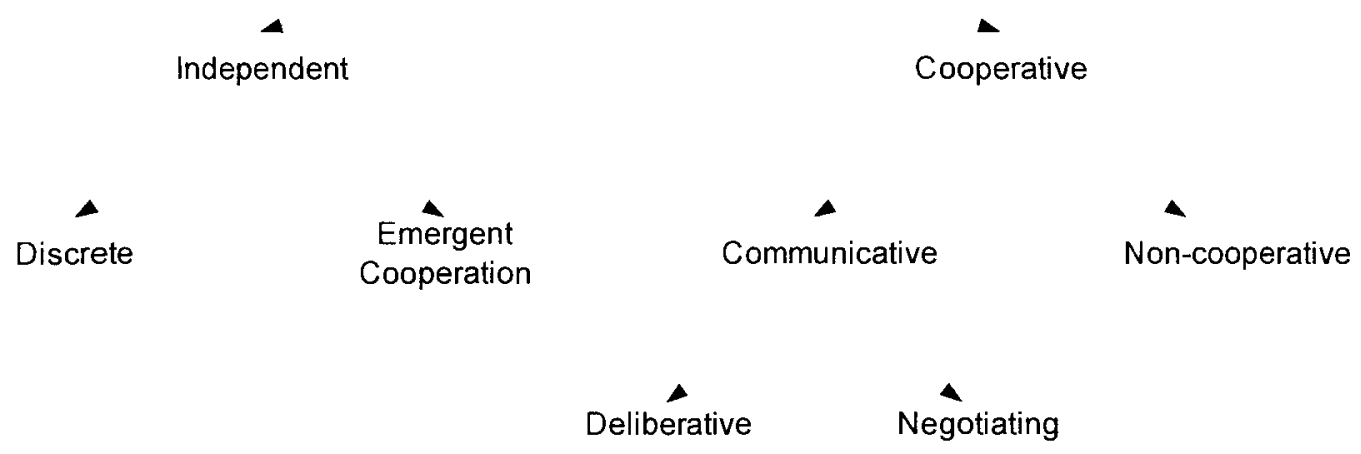

Figure 2-5: Cooperation typology

Enabling the cooperation concepts is the development of individual agent autonomy, and the capability of having interactions between agents. In 1962 Thomas Kuhn, author of "The Structure of Scientific Revolution", first defined the concept of a paradigm shift when he argued that scientific advancement is not evolutionary, but rather is a "series of peaceful interludes punctuated by intellectually violent revolutions", and in those revolutions "one conceptual world view is replaced by another". With MAS comes a new revolution in the design of agent systems: away from classical AI and towards emergent behaviour. Today we are still in the middle of the intellectually violent revolutions. According to Ferber (1999) there are two major objectives being pursued in research today. The first is the theoretical and experimental analysis of self-organization mechanisms that occur when several agents interact. The second is the creation of distributed systems that are capable of interacting and cooperating to perform complex tasks. 
There are often limited resources in the areas where UAVs are used, including low bandwidths available for communication, geographical separation between vehicles that are operating in a constantly changing environment, and a lack of aircraft situational awareness by the ground control station operators. This situation is an ideal implementation environment for multi-agent systems, which are capable of:

- Planning or reacting to achieve goals,

- Modelling their environment,

- Sensing and acting,

- Coordinating among the agents, and

- Resolving conflicts.

These capabilities are referred to as the core competencies, which were first introduced by Barber and Lam (2001).

\section{$\underline{2.3 .3 \text { Multi-Agent Svstems (MAS) Definitions }}$}

MAS is a term applied to a system comprised of the following elements (Ferber, 1999):

- An environment, E, is a space which generally has a volume.

- A set of objects, O, that are situated within the environment. Therefore, it is possible at a given moment to associate any object with a position in E. The objects are passive, that is, they can be perceived, created, destroyed and modified by the agents. 
- An assembly of agents, A, which are specified objects, representing the active entities of the system.

- An assembly of relations, which link objects (and thus agents) to each other

- An assembly of operations that make it possible for the agents to perceive, produce, consume, transform and manipulate objects.

- Operators with the task of representing the application of the operations, along with the world's reaction to the attempts at modifications, are called the laws of the universe.

\subsubsection{Multi-Agent Systems (MAS) Design Elements}

Multi-agent based systems may either be heterogeneous with each agent responsible for a different set of goals or homogeneous where the agents share the same goals. MAS design consists of dividing resources and domain responsibilities among agents, determining which core competencies satisfy which domain responsibilities, and selecting the AI techniques to satisfy each core competency (Barber et al., 2003). The architecture and relationships among the agents within MAS can be likened to the organizational structure found in nature, with layers embedded within each other. For example, consider a single cell found in biology, where each component within that individual cell has a task, yet the cell itself is just one of many that interact to form a multi-cellular organism.

The organization of each agent within MAS has been analyzed with reference to five dimensions as illustrated by Figure 2-6. 


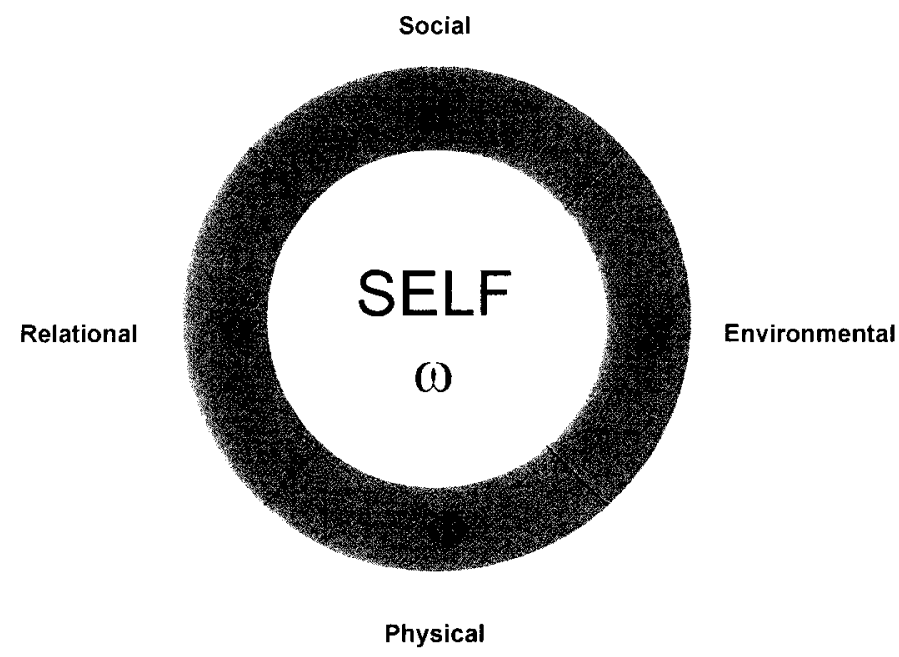

Figure 2-6: Five dimensions of an organization (Ferber, 1999)

The physical dimension of an organization examines explicitly how a task should be carried out within the organization, while the social dimension is concerned with how the activities of the organization fit within the higher-level structure. The relational dimension is concerned with the interactions between two or more different organizations. It asks behavioral questions: with whom, for what reason, and how, should the organizations interact? The environmental dimension is tied in with the ability to act, perceive and reason about actions within its surrounding world. The personal dimension is primarily interested in acquiring knowledge and preserving its structure. Consideration for all five dimensions depends on the type of analysis that is required. For example, a reactive organization requires only physical and environmental dimensions, whereas a communicative organization requires physical, social, relational and personal dimensions. Whether it is a reactive or communicative organization depends entirely on the implementation environment of the design and its purpose. 


\subsubsection{Multi-Agent Systems (MAS) Design Process}

A successful multi-agent design requires the deconstruction of a problem into smaller sub-problems before autonomous agents can engage in flexible, high-level interactions. Solving a problem in this fashion leads to an outcome, which ensures that the final solution addresses the original, often complex, engineering problem. The desired result is a final solution that is distributed, with multiple loci of control. This decentralized framework increases the system's modularity and results in a lower coupling among components. Using a distributed approach means that rather than having monolithic agent structures perform all of the tasks, the problem is broken into smaller pieces that can be handled by several interconnected agents, similar to peer-to-peer networks. While several agents can be linked together in some way, it is desirable to avoid a 'star' configuration whereby all agents communicate via a single agent. Depending on the frequency and amount of information being passed among agents, this central agent represents a potential bottleneck in the system. The self-awareness of individual agents reduces the need for a centralized repository of control know-how, and benefits from each agent's ability to make localized decisions and act on these decisions.

Similarly, rather than trying to predict all possible interactions during the design phase, it is easier to endow the agents with the ability to make decisions about the nature and scope of their interactions. This means that individual agents are able to request additional information from other agents in order to supplement their current knowledge before making a decision. For a programmer, this reduces the need to anticipate all possible interactions that will occur at run-time. Therefore it is important to understand 
that at any one time, all agents within a multi-agent solution are active, and coordination is handled through inter-agent communication (Jennings, 1999).

Coordinating the exchange of this information requires a discussion of coordination techniques. One possible technique uses a leader-follower configuration. Here all decisions are made by the leader, which avoids unnecessary communication for establishing decisions. However this system is fragile because of its reliance on a single leader. If vehicles must perform a coordinated re-planning, this may lead to uncoordinated activity because each vehicle's local response might be conflicting (Durfee, 1998). Durfee suggests that it is important that the collective group of agents knows enough about the collective goals, and not just the individual goals, in order to forge ahead. In practical applications, vehicles may only be in communication with the ground intermittently and it is important to communicate intent.

\subsubsection{Agents and $U A V S$}

Agent oriented programming recently demonstrated that it is suited for making path planning decisions within a UAV flying in an environment with changing wind conditions. While the concepts of use have existed for quite a few years, it was only in July 2004 that defence researchers at DSTO in Australia demonstrated the world's first, in-flight intelligent agent control while flying Codarra's Avatar UAV (Park and Williams, 2004). In this exercise, researchers used the JACK software package from Agent-Oriented Software Pty. Ltd. (AOS). JACK is a BDI-agent based program that was initially developed for the computer simulation of military tactics and equipment (Lucas 
et al., 2003). When used onboard the UAV, the intelligent agents choose a flight path, after evaluating real-time flight and weather data. This increase in autonomy demonstrated that it is possible for a UAV to autonomously re-plan and execute a mission without ground operator intervention. The use of intelligent agents advances the state of the art within the system architecture, as it is used with the existing systems onboard a UAV to provide the oversight and control required for truly autonomous flight. Agent oriented programming is not suitable for all aspects of controlling a UAV. An autopilot system includes a series of proportional-integral-derivative loops (PID controller), whereas agents are applicable at the highest level of abstraction within the pyramid structure presented by Lucas et al. (2003), as shown in Figure 2-7. Following the figure, each level is discussed.

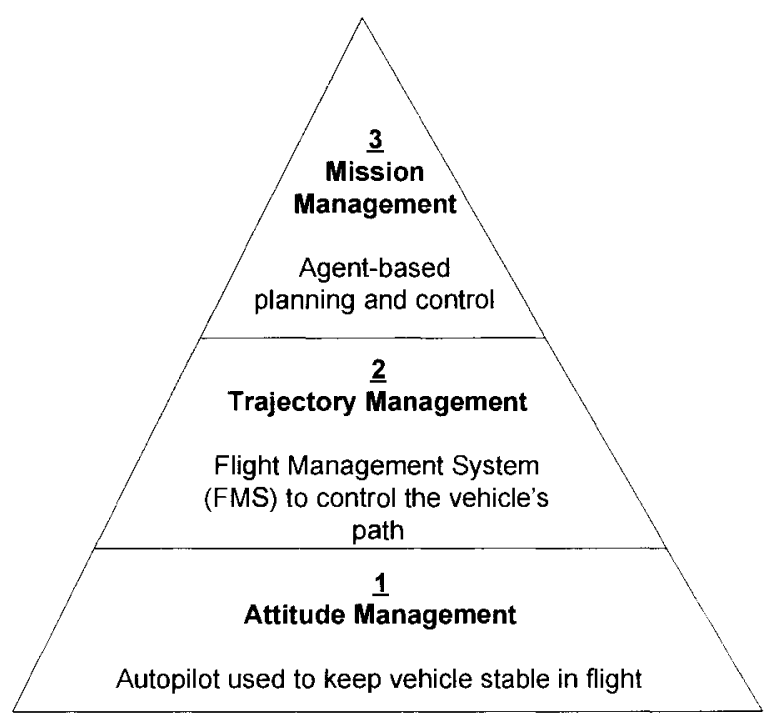

Figure 2-7: Vehicle control hierarchy using agents

Tier 1: Autopilot layer: concerned with keeping the aircraft on a given course, altitude and speed.

Tier 2: Flight Management System layer: concerned with trajectory management, flying the aircraft along a multi-vertex path. 
Tier 3: Mission management layer, where decisions about how to achieve an objective are made. This tier is programmed according to the doctrine of operations for a particular $\mathrm{UAV}$; in this case, search and rescue.

At the lowest control level, the agent steers the UAV by issuing instructions to the autopilot. The intelligent agent, which incorporates a BDI execution mechanism, carries out higher-level behaviour tactics expressed by the range of BDI plans, which will change depending on the situation.

As part of the evaluation process, a workshop with AOS was organized at Carleton University. Workshop attendees included members from DRDC, along with a number of local area companies who had also indicated that they were currently using JACK intelligent agents. Aside from local users, JACK is also in use at a number of universities around the world, as well as the defence research centres in Australia, and the UK. Given the suitability of multi-agent programs for making real-time decisions within a dynamic environment, and the wide-spread adoption of the program by other users, the decision was made to use the JACK software for the SAR demonstration within this thesis.

Agent Oriented Software Pty. (AOS) has also developed a software framework that deals with teaming. Each agent in the team has individual goals and capabilities, and the collection of agents have group goals that leverage the abilities of the individual agents. In this configuration there is one leader with multiple subordinates; however, if the leader drops out of the team, another agent with appropriate capabilities can take control. The 
concepts of teaming could be applied in the future to controlling a group of UAVs, where each vehicle is controlled by a set of individual agents.

The challenge of coordinating teams of UAVs is daunting. It is possible that one agent deals with the individual control of the vehicle and one agent deals with group control. Assuming each vehicle within a team has a similar configuration of internal agents, then the multiple proxy agents could monitor their local states while also providing some of this local representation to the other UAVs. While AOS currently provides the tools to handle teams of agents, the underlying theory of coordinated activities requires additional study beyond the scope of this thesis. For example, if all the air vehicles within a team have the same capabilities, how should the group self-organize in order to accomplish the team goal? While this thesis focuses solely on the control of only one vehicle, the possibilities afforded by teaming, warrant future exploration.

\subsubsection{Common Pitfalls of Agent Programming}

This section is based on the findings of Wooldridge and Jennings (1998), and Winikoff, Padgham, and Harland (2001), all of whom are AI practitioners and professors of intelligent agent programming. These practitioners found that programmers new to intelligent agents often have problems breaking the program down into the necessary smaller pieces and instead opt to build monolithic programs that try to internally handle every possible contingency plan (Winikoff et al.,2001). Instead, a distributed approach is more suitable with the development of plans that could be applied in different contexts. This approach leverages the advantages of agent-based decision making, which will first 
determine, and later invoke, the most appropriate plan. It is important to remember that the basic essence of intelligent agents is rational decision making. Therefore it is key that agents are able to answer a number of generic, non-application-specific questions such as: Which action should I perform? Which goal should I work on? How should I realize this goal? Where shall I go? With whom should I interact? All of these questions must have non-trivial answers. If an agent only has one goal at a time, and a single means of realizing this goal, then there is no opportunity for decision-making or flexible intelligent behaviour. Winikoff, Padgham and Harland (2001) also found that new practitioners often have significant difficulty in interfacing the agent to its environment.

Wooldridge and Jennings (1998) suggest that concurrency is one of the most important potential advantages of multi-agent solutions and one that is seldom exploited. Concurrency allows the system to simultaneously handle multiple objectives and perspectives to respond and react to the environment at multiple levels. This results in many levels of problem solving with complementary methods working together. The challenge to the practitioner who lacks formal training in distributed artificial intelligence, is trying to establish the framework for evolving a system in this environment.

It is agreed upon by all five cited experts that the lack of formal, widely used design methodologies is one of the greatest challenges facing the intelligent agent community. 


\subsection{Applicable Standards}

One of the major challenges facing the development of UAV systems is the historic lack of standards designed for UAVs. Without such standards for airframes, engines, avionics, or other systems each manufacturer's design is unique. Furthermore, few UAV manufacturers have followed the safety or airworthiness guidelines for manned aircraft. As a result, civil transportation authorities around the world are currently grappling with ways to regulate UAV activities. Presently, a number of working groups exist in Europe, the United States and Australia.

This section summarizes the specifics of both RTCA DO-178B "Software Considerations in Airborne Systems and Equipment Certification", and STANAG 4586 "Standard Interfaces of UAV Control System for NATO UAV Interoperability". Both standards were consulted during the development of the intelligent agent software because the agent software augments the current interface to a UAV's control system, and represents a safety critical piece of software. This information is referred to in the agent-based program described in Chapter 6 of this thesis.

\section{$\underline{2.4 .1 R T C A D O-178 B}$}

In the early 1980s the RTCA, an association of government and industry aeronautical organizations of the United States, acknowledged the need for industry-accepted guidelines that would satisfy airworthiness requirements for software in airborne systems. DO-178B "Software Considerations in Airborne Systems and Equipment Certification" was written to satisfy this need (RTCA, 1992). Following discussions between the author 
and Squadron Leader R. Grainger (personal communications, 1 Oct 2004), Squadron Leader Grainger spoke to the importance of following the RTCA DO-178B standards when developing software for use within a UAV system. This document is not a military standard, nor does following the guidelines ensure certification by flight authorities.

While the information contained below originated from RTCA DO-178B, full compliance would require both time and financial resources that are beyond the scope of this academic project. Based on information provided by Capt. S. Sterling, (personal communication, 1 Oct 2004) there are very few commercial, off-the-shelf systems available which have been developed in full accordance with DO-178B. Often a system baseline is determined, and then this baseline is compared with the standard before the criticalities of the differences are noted. Depending on these differences, flight restrictions may be placed on the vehicle. The purpose of this section is to provide general guidance that encourages software development practices that are consistent with DO-178B.

Introduction of DO-178B is appropriate because the standard defines steps in the software development process including documentation of system requirements, hardware interface and system architectures, software development plan, and software requirement standards. 
As per Section 5.0 of DO-178B, the software development processes include:

Software requirements process: objectives include developing high-level requirements, and indicating which high-level requirements are relevant to the safety assessment process. Consideration for safety standards at the early phase of the programme ensures that safety is an integral component during the design and implementation phases. This means understanding the severity of a failure condition on the UAV system is of paramount interest. It is important to identify areas where an error in the software may indirectly lead to a failure condition. From a safety perspective, software is divided into five levels defined by (RTCA, 1992); the two most significant are defined as follows:

Level A: Software whose anomalous behaviour, as shown by the system safety assessment process, would cause or contribute to a failure of system function resulting in a catastrophic failure condition of the aircraft. (Catastrophic: Failure conditions which would prevent continued safe flight and landing.)

Level B: Software whose anomalous behaviour, as shown by the system safety assessment process, would cause or contribute to a failure of system function resulting in a hazardous/severe-major failure condition for the aircraft. (Hazardous / Severe-Major: Failure conditions which would reduce the capability of the aircraft or the ability of the crew to cope with adverse operating conditions to the extent that there would be: a large reduction in safety margins or functional 
capabilities, physical distress or higher workloads such that the flight crew could not be relied on to perform their task accurately or completely.)

Software design process: low-level requirements and software architectures are developed conforming to software design standards including traceability, verification and consistency. Any derived requirements should be defined and analyzed to ensure that the higher-level requirements are not compromised. Within the software design process it is also important to flag possible modes of failure of the software or, conversely, preclude others. The use of partitioning or other architectural means in the software design may alter the software level assignment for some components of the software. Responses to failure conditions should be consistent with the safety-related requirements.

Software coding process: the developed source code must be traceable, verifiable, consistent, and implemented correctly to satisfy low-level requirements.

Software testing processes are outlined in Section 6.4 of DO-178B, including details of hardware/software integration testing, software integration testing and low-level testing. It is recommended that during such testing the software be loaded into the target computer and tested in a high fidelity simulation of the target computer environment (RTCA, 1992). It is for these reasons that details of the components within the simulation environment are presented in Chapter 7 of this thesis. The necessary software design standards and quality assurance recommendations are covered under Section 11.5 though 
11.11 of DO-178B. Tables Al through A10 of DO-178B guide the reader through the necessary steps to ensure that all recommendations are met.

\subsubsection{STANAG 4586}

On the 11th April 2003, the office of the Deputy Chief of the Defence Staff signed the ratification document for the NATO standard STANAG 4586 "Standard Interfaces of UAV Control System for NATO Interoperability", making this the standard for UAV interoperability in the Canadian military (J. Gobin, personal communication, 8 March 2005). Because aerial SAR missions are flown by the Canadian Forces, any workable solution involving UAVs must be consistent with existing architectures. A high-level depiction of this architecture is found in STANAG 4586, and is shown in Figure 2-8. Information from the air vehicle is sent back to the Vehicle Specific Module (VSM) using either line of sight (LOS) or beyond line of sight (BLOS) communications. When the data reaches the VSM, details that are particular to the individual UAV are interpreted before the information in passed over the Data Link Interface (DLI) to the Ground Control Station (GCS). 


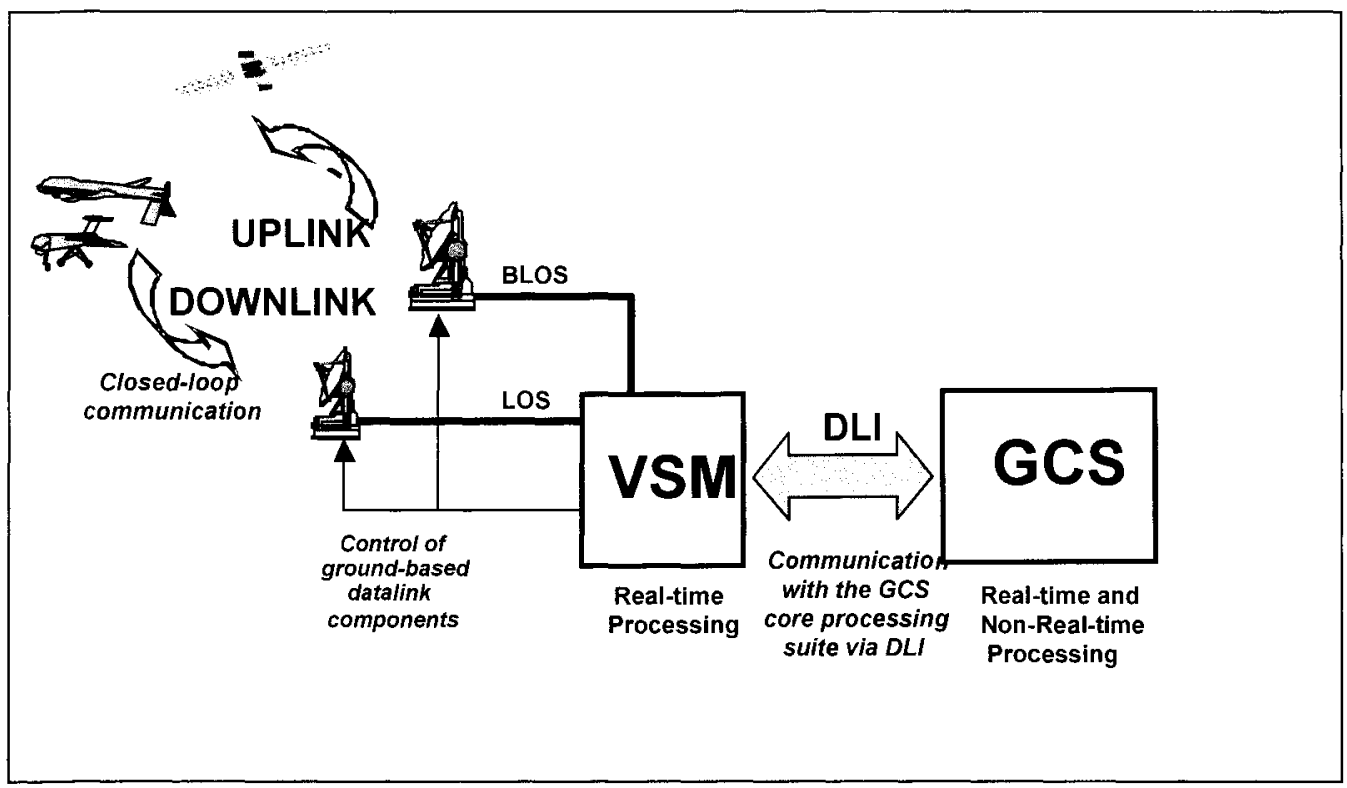

Figure 2-8: Role of the VSM

Figure 2-9 examines the type of information that is passed between the air vehicle and the control station. Analogue data and voice communications are passed to the human computer interface specific module (HCISM) and the command and control interface specific module (CCISM), while all digital data is passed directly to the GCS, and displayed to the human operators. 


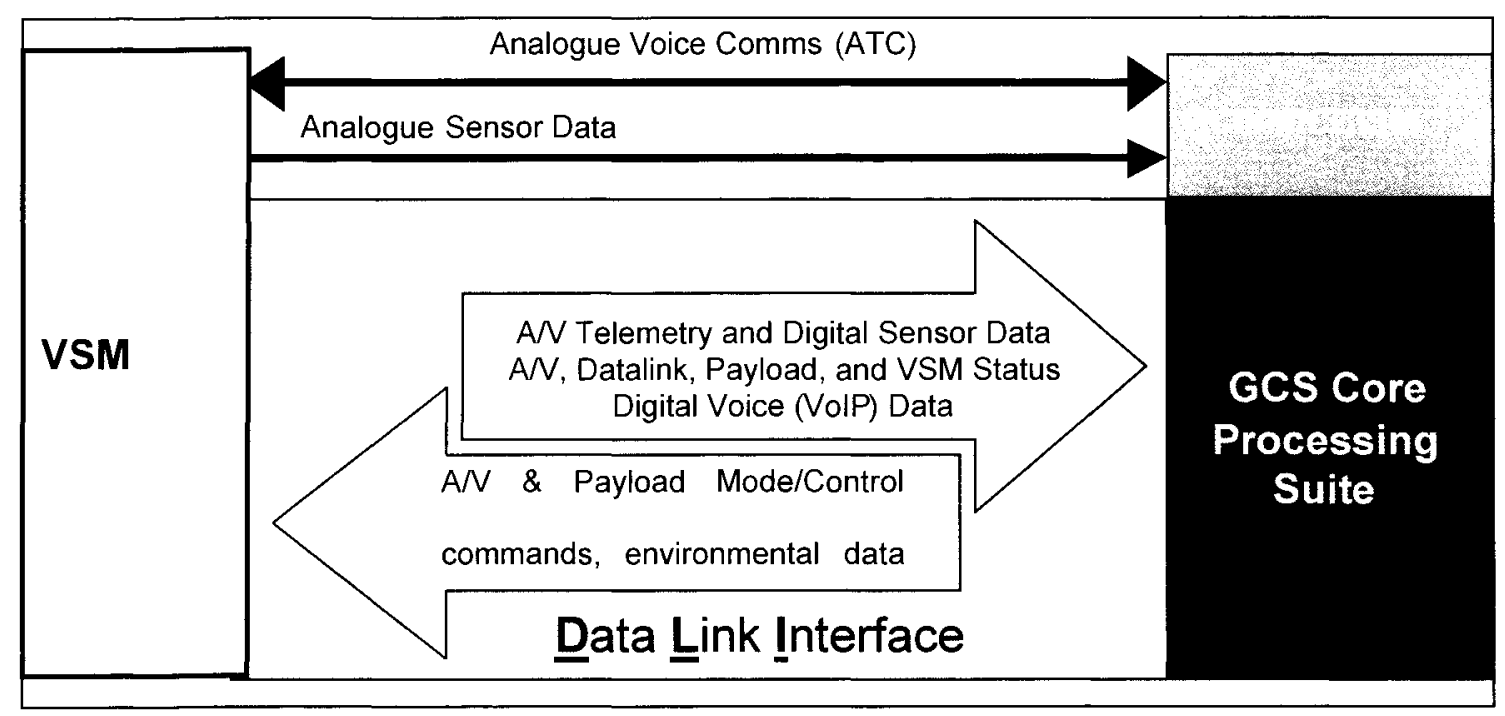

Figure 2-9: High-Level Depiction of DLI Interface Content

Following the development of the agent program in Chapter 6 of this thesis, Figure 2-9 is modified and again referenced as the use of agents within a UAV affects STANAG 4586. 


\section{Chapter 3: Search and}

\section{Rescue Scenario}

\section{CHAPTER SUMMARY}

A search and rescue scenario was chosen for the demonstration of intelligent agent approach because of its importance to Canadians, and because the scenario shows a potential value to both civilian and military users of unmanned air vehicle technologies. The SAR scenario was developed through numerous consultations along with a visit with the search and rescue personnel from the Joint Rescue and Coordination Centre in Trenton Ontario. As presented, the SAR scenario also includes a concept of operation for both the conventional search assets and unmanned air vehicles. 


\subsection{Search and Rescue Scenario}

In 1979, R.P. Hypher of the Canadian Forces Air Transport Headquarters, wrote a report on the concentration areas of air accidents in Canada, and highlighted that a number of incidents occur in the middle north to arctic regions. The locations of incidents over a five-year period, prior to 1979 are shown in Figure 3-1.

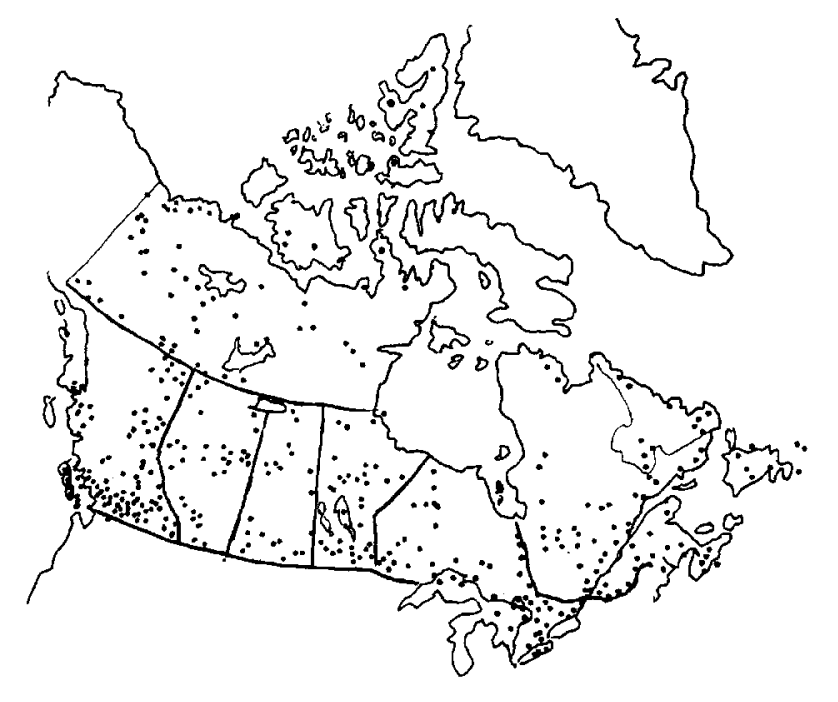

Figure 3-1: Aircraft accidents locations in Canada

Hypher noted that $68 \%$ of all crashes and forced landings involved single engine, fixed wing aircraft under $2,000 \mathrm{~kg}$ in weight, with the vast majority of these aircraft being privately owned. Of the aircraft that crashed, $93 \%$ had five or fewer people on board; however, fatalities occurred in about half of the air distress cases. Furthermore, Robert Mattson (1979) of the USAF noted that $60 \%$ of all survivors of an aircraft crash are injured, and after 24 hours only $20 \%$ of the injured are still alive. In accidents involving fatalities, five out of six of those on board do not survive. Mattson stated that the probability of finding an uninjured survivor alive after 72 hours is very small. This clearly highlights the importance and time-criticality of finding a missing aircraft. 
Hypher reported that approximately half of the nonfatal crashes are located within 11 hours and $75 \%$ of cases are located within 20 hours after time of notification. However these numbers hide the fact that notification time and the actual time of the emergency may not be the same, as it is sometimes days before it is known that an aircraft is missing.

Considering Hypher's work, a scenario of a missing aircraft has been chosen to demonstrate a possible application of UAVs assisting in the search and identification of the missing parties, alongside conventional search and rescue assets. For the purposes of this research project, use of only one UAV is considered, however, the scenario is equally suited for multiple UAVs. The scenario has been adapted from the 1994 White Paper on Defence (Chief of the Air Staff, 1994), which forms the basis of Canadian Defence Policy. The search and rescue scenario for a missing aircraft was chosen and subsequently updated and modified through consultation with JRCC Trenton (Stockermans, Bernier, Gray, personal communication with the author, 1 Sept 2004).

\subsection{Overdue Aircraft Scenario}

The following scenario was developed for the purpose of designing a UAV test situation. In late September, a resident of St. Albert (NW of Edmonton) Alberta flies to his lodge located on the north shore of Lesser Slave Lake for a five day hunting trip. He is travelling with two friends using his Piper Super Cub float-equipped aircraft. The hunters' intentions are to use the aircraft to transit among hunting sites around the Lake and surrounding area (see Figure 3-2). No specific flight plans are filed for these ad hoc transits. A flight itinerary was filed such that in the event that the pilot failed to meet his 
estimated time of arrival (ETA), the Joint Rescue Coordination Centre (JRCC) would be notified one hour after the missed ETA.

In the afternoon (1400 hrs local) of the sixth day of the trip, JRCC Trenton is notified of the overdue aircraft, when Edmonton Flight Information Centre (FIC) files an information request (INREQ). Edmonton is one of the 9 FICs in Canada, and holds flight plans and flight itineraries for all flights in its region. A communications check for the aircraft confirms that it had arrived at Lesser Slave Lake on day one, refuelling at the east end of Slave Lake $(\mathrm{CYZH})$. Edmonton FIC has no report of the aircraft departing as planned on the morning of the sixth day. Canadian Mission Control Centre (CMCC) is tasked to monitor the area around Lesser Slave Lake and the route to St. Albert for an Emergency Locator Transmitter (ELT) signal. An alert notice (ALNOT) is issued one hour after the INREQ. As the communications search is unsuccessful at locating the aircraft, at $1530 \mathrm{hrs}$ on the sixth day JRCC Trenton tasks rescue aircraft to begin searching for the missing aircraft. At midnight (local) a missing aircraft notice (MANOT) is issued. By $1800 \mathrm{hrs}$ (local) on the seventh day, control for the search is passed to a search headquarters that has been established at the Slave Lake airport.

The searchmaster has assumed that based on the available information, the area to be searched is Lesser Slave Lake and the surrounding lakes (where the pilot normally goes to fish/hunt) as well as his route back to Edmonton. To define a search area around the lakes, JRCC Trenton seeks out whatever information is available from friends and relatives about where he would normally go to hunt or fish. With no other information 
available, the search area is defined as a circle, centered at Lesser Slave Lake, with a radius based on the aircraft's endurance.

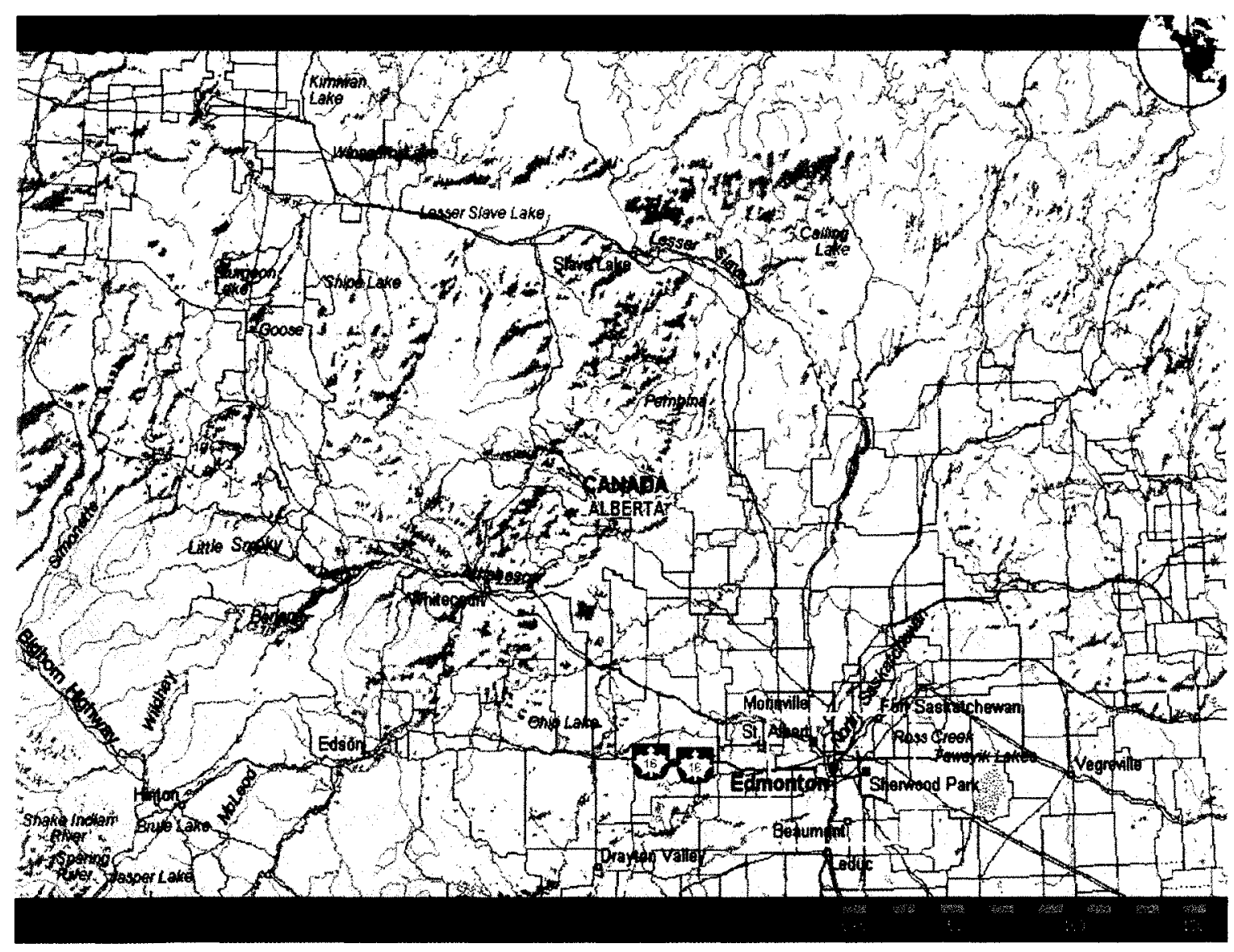

Figure 3-2: Missing hunting party - area of operation

\subsection{Intelligence}

\subsubsection{Political}

This incident has occurred within the Canadian SAR area of responsibility. The Canadian Forces will coordinate, control, and conduct the search and rescue effort for the missing aircraft to minimize the loss of life. 


\subsubsection{Sociological}

The area immediately to the north of Edmonton is sparsely populated with many small English-speaking communities. As one proceeds further north beyond the Athabasca River towards Lesser Slave Lake, the number and density of small communities diminishes dramatically. The area immediately to the north and south of Lesser Slave Lake is virtually unpopulated.

\section{$\underline{\text { 3.2.3 Geography }}$}

\subsubsection{Topology}

The dominant landform throughout the region is prairie plain covered with boreal forest. The region to the southwest of Lesser Slave Lake is hilly, being the beginning of the foothills to the Rocky Mountains.

\section{$\underline{\text { 3.2.3.2 Hydrography }}$}

Lesser Slave Lake is a fresh water lake spanning approximately $90 \mathrm{~km}$ by $10 \mathrm{~km}$. The major river flowing through the area is the Athabasca River originating in the Rockies to the West and flowing to Lake Athabasca in the northeast of Alberta.

\subsubsection{Infrastructure}

\section{$\underline{\text { 3.2.4.1 Transportation }}$}

There is a widespread road network to the south of the region. Roads become fewer and more widely dispersed north of the Athabasca River. All roads are unpaved, secondary gravel highways. 


\subsubsection{Airports}

There are several small airfields and flight service stations throughout the region. Most have runway lengths capable of handling CC-130 aircraft movements.

\subsubsection{Communications}

There is a rudimentary telecommunication system throughout the area with widespread use of citizen band radio transmitter-receivers.

\section{$\underline{\text { 3.3 Miscellaneous Factors }}$}

The situations where small aircraft go missing occur throughout Canada, and most incidents are resolved without a major search being required. However, several major searches are conducted every year. Weather conditions and terrain could impose restrictions and constraints on how each operation is conducted.

It is assumed that the missing parties are cooperative and want to be found. They may be stationary or moving. Once the whole area has been covered during the first day search for the cooperative survivors, it is assumed that the search object is non-cooperative, perhaps due to illness or injury. It is also assumed that the missing parties may require immediate medical assistance.

\subsection{Concept of Operation}

Upon notification, the JRCC will conduct a communications search to locate the missing aircraft. At the same time the CMCC will be tasked to monitor for ELT signals in the 
region in question. If these attempts fail to locate the aircraft, SAR search assets including a UAV will deploy and conduct a visual and electronic search following Canadian search procedures. Once the crash site is located, SAR technicians will provide first aid to the survivors, as required. Survivors will then be transported to the nearest suitable medical facility to receive any necessary medical treatment.

If the crash site cannot be located, the search will be reduced when it is determined that there is no reasonable hope of locating survivors.

\subsubsection{CF Contingent Concept}

The Joint Rescue Coordination Centre (JRCC) Trenton will initiate the SAR operation. SAR fixed-wing aircraft such as a CC-130 carrying a mobile ground control station (GCS), launch system, and an unmanned aerial vehicle (UAV) will be launched from the nearest available base. If the missing aircraft cannot be located following initial communications and physical searches, a searchmaster will be designated. The searchmaster will establish a headquarters in the vicinity of the search region and the GCS will be located adjacent to this local headquarters. The searchmaster will control the search assets that have been tasked and assigned to the search. He/she will, on a daily basis, define the areas to be searched and assign the search areas to the various search assets. The UAV operator and payload specialist will upload the appropriate information to the UAV asset. If the aircraft and hunters are located, SAR Technicians will deploy and provide immediate medical aid as required. 


\section{$\underline{3.4 .2 \text { Interoperability }}$}

Coordination with civil aviation and marine resources may be required, as well as civil authorities. CASARA aircraft will support the air search and local marine vessels or the RCMP could conduct a search of the lake area. Land search parties, formed from RCMP, volunteer or deployed CF personnel (Ground Search Teams) could be organized to participate in the search.

\subsubsection{Sustainment Requirements}

The deployed CF units would be sustained for the duration of the operation. Each major SAR search is unique and the duration is determined by a variety of factors. However, very few major searches in the past have lasted beyond 20 days (Stockerman, 2004).

\subsection{End State Conditions}

The operation would conclude when either of the conditions below occurs:

The crash site and/or hunters are located, injured survivors have been evacuated to a medical facility and fatalities have been turned over to the district coroner. The SAR case is then closed by the JRCC.

The crash site or hunters have not been located and, in the opinion of the searchmaster, there is no reasonable chance of locating survivors. The search master's recommendation to reduce the search is accepted. In this case, active searching by the $\mathrm{CF}$ ceases but the file is kept open until the crash site and/or missing persons are located. The case is then handed over to the RCMP as a missing person. 


\section{$\underline{\text { 3.6 Search Assets }}$}

\subsubsection{Conventional Aircraft}

Available fixed wing assets include two or three CC-130 Hercules with any available CASARA members providing support aircraft. These aircraft will search at higher altitudes in the periphery regions and monitor emergency radio channels. These assets will fly according to the CSAD 1 and 2 areas, along the planned flight route at altitudes of $1500 \mathrm{ft}, 1000 \mathrm{ft}, 500 \mathrm{ft}$ at track spacings of 6 nautical miles $(\mathrm{nm}), 2 \mathrm{~nm}$, and $1 \mathrm{~nm}$ respectively. Once the other areas around Lesser Slave Lake are defined they too would be searched at these altitudes and track spacings. Rotary wing assets include two or three CH-146 Griffon helicopters dispatched from CFB Cold Lake and possibly a $\mathrm{CH}-149$ Cormorant helicopter from CFB Comox. These aircraft will perform concentrated searches at slow speeds and will be used in the evacuation of casualties and/or recovery of SAR technicians.

\subsubsection{Unmanned Aerial Vehicles}

A medium altitude long endurance (MALE) UAV will be tasked to monitor the entire region and will cue (green arrow in Figure 25) smaller UAV(s) to high probability areas. After being cued, the smaller UAV executes a search at low altitude. The MALE UAV will also be equipped as a communications platform with a radio repeater to enable line of sight communication with all search assets. At the present time only the use of one UAV is being considered for the low altitude search. However, future research may lead to the inclusion of multiple UAVs searching different areas. 
Depending on the chosen platform, most UAVs are capable of carrying one or more of the following payloads: EO/IR video camera, hyper-spectral or high-resolution singleimage digital camera. It was suggested by Sgt. G. Gray (personal communications with the author, 1 Sept 2004) that a hemispherical camera also be carried to scan for smoke or flares beyond the swath region. Each vehicle communicates information obtained by the sensors to the ground control station (GCS). Depending on the onboard computing capabilities, it may be possible for the MALE UAV to cue the smaller assets directly; however, the command would also be sent to the GCS to inform the operator of the planned actions. Two possible UAV systems may be deployed for the search mission.

The CQ-10A SnowGoose is a GPS guided para-foil system with a maximum range of $300 \mathrm{~km}$ and 12 hour endurance while carrying up a maximum payload of $275 \mathrm{~kg}$ of payload. A maximum endurance of 20 hours is possible when fuel is substituted for payload capacity. It has a cruise speed of approximately $65 \mathrm{~km} / \mathrm{h}$ and is capable of flying up to a maximum ceiling of $5500 \mathrm{~m}$. The SnowGoose may be aerially deployed from a CC-130 or launched from the back of a truck driving down a runway. It requires no more than a heli-pad sized area for landing.

The Manta B UAV from Advanced Ceramics Research is a small fixed-wing UAV with a $2.67 \mathrm{~m}$ wingspan. It has a cruise speed of 65 to $130 \mathrm{~km} / \mathrm{h}$ and endurance of five to seven hours with a maximum ceiling of $4870 \mathrm{~m}$. The Manta $\mathrm{B}$ requires either a pneumatic launcher or short runway for takeoff. This system can carry up to $7 \mathrm{~kg}$ in payload for 
sensors, and cameras and uses the L-Band frequency for transmitting video at a range of 14 to 18 nautical miles, line of sight.

\subsection{Scenario Evolution}

By working in the fully networked environment, any of the primary search assets CC-130, CH-146, CH-149 or the MALE UAV- are capable of cueing the smaller UAVs to a region that warrants closer investigation. The coordinates and error estimate for the region are also sent to the searchmaster and GCS operators. However, flying the UAVS in the same airspace as manned assets is a safety concern for the searchmaster (Bernier, 2004). Therefore the UAVs will be assigned to a search quadrant during the daylight hours, similar to the tasking of CASARA aircraft. The UAVs will also execute cueing instructions at night when the airspace is clear of other aircraft. After receiving instructions and permission from the GCS to proceed to the probable area, the small UAV must execute a search of the area, detect debris from the aircraft, geo-locate the missing aircraft's position and determine if there are any survivors. A high-resolution image of the site will be taken and sent to the GCS at which time the searchmaster will determine the next step of the mission in coordinating rescue efforts. All search efforts will be made in accordance with common operating procedures for Canadian SAR activities as defined in the National SAR Manual and Searchmaster's handbook.

Currently, researchers at DRDC and elsewhere are exploring ways for individual UAV operators to control multiple vehicles and their payloads at the same time. However, to reduce operator workload, the organization, search, and identification process requires a 
degree of onboard intelligence within each UAV. This intelligence is based on a set of pre-defined rules and is used to control the air vehicle's emergent flight behaviour and sensor position. Throughout this autonomous portion of the mission, the intentions of each vehicle must be made known to the ground operators (red arrow of Figure 3-3), who at any point in time must have the ability to abort the action and take control of the vehicle.

The SAR mission benefits from the fully networked environment and the coordination of multiple autonomous vehicles working alongside existing SAR aircraft through one single point and sharing the gathered information. This scenario forms the basis for the experiment described in Chapter 7. 


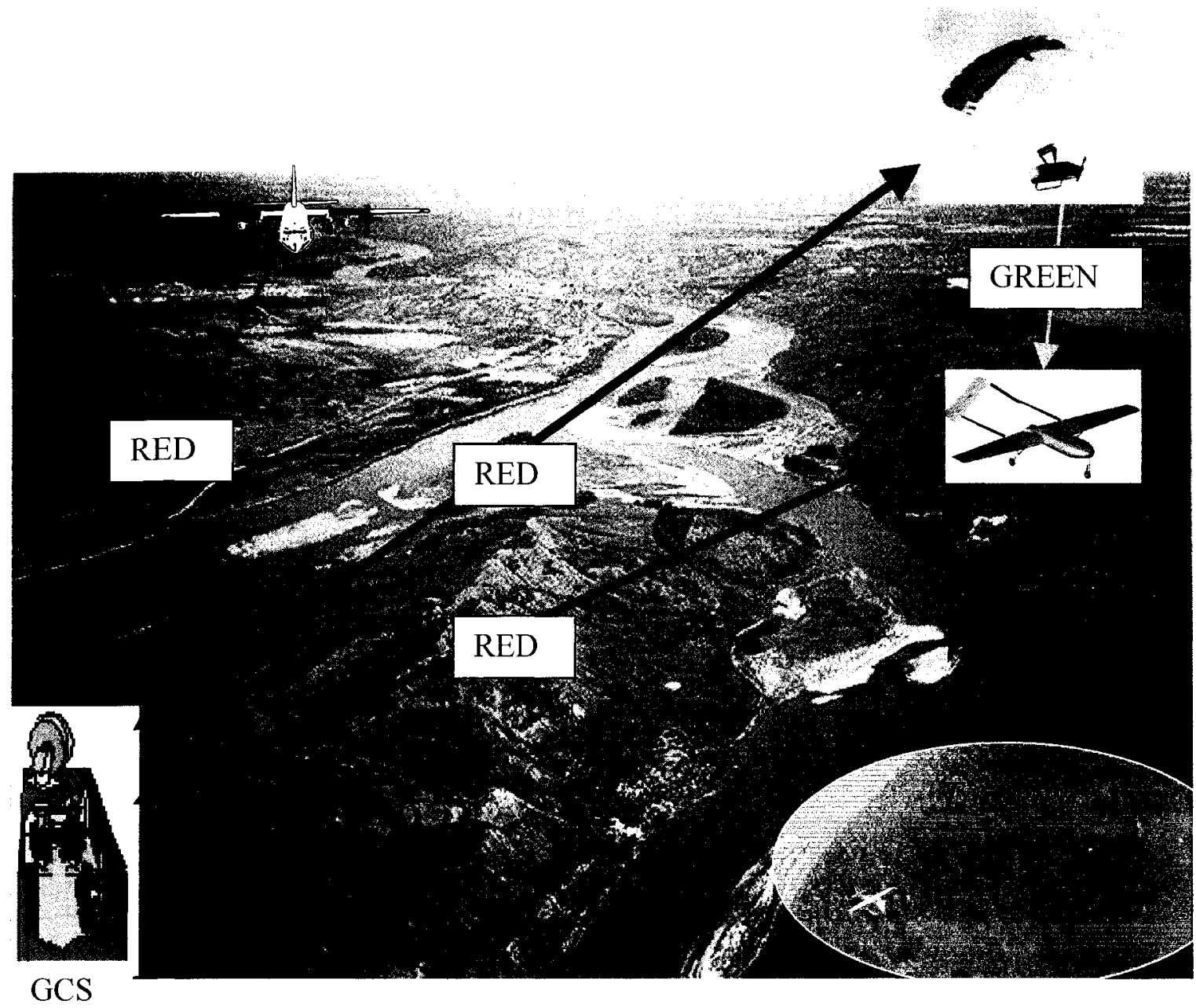

Figure 3-3: Mission coordination through GCS and cueing between UAV platforms 


\section{Chapter 4: JACK}

\section{Intelligent Agents}

\section{Chapter Summary}

Intelligent agents form the cornerstone for this thesis. Using a software development environment called JACK, an intelligent agent programme is created. Within this chapter, the individual components of the programme and the design methodologies of a multi-agent system are discussed. A brief description of communication methods between individual agents is also provided. 


\subsection{JACK Intelligent Agents}

This section provides an overview of the elements of the Design Tool menu of the JACK Development Environment. Within a JACK program there are six pre-configured elements: Agents, Capabilities, Events, Plans, Named Data, and Notes. In addition to these design elements, a programmer is also able to create custom elements by programming them in JAVA and importing the code into JACK. Information for this section was obtained through documents supplied by Agent Oriented Software. These included JACK Intelligent Agents User Guide, Development Environment Manual, and Design Tool Manual (AOS, 2004).

\subsection{Components of Multi-Agent Systems}

\subsubsection{Agents}

As primary decision makers in the system, agents require the ability to handle, process and post information. Details about the agent's goals and environment are obtained from beliefsets while events (both internal and external) can supplement this information. Each agent has a set of beliefs about the world and, depending on the application, these data sets may be quite limited. Due to the distributed nature of multi-agent programming, each agent can be a specialist in one area rather than having a holistic view of the world. Each agent only responds to a particular set of events (triggers) and uses a set of plans in order to achieve its goals. In doing so, the agent may be required to post events internally that are handled by other plans, or post events externally that are handled by other agents. 
In a way, an agent is similar to an operator with a procedures manual. Agents include the rational behaviour of an operator, which distinguishes it from just being an expert system. The goal-focused approach puts the emphasis on the final objective, rather than the process to achieve it. Agents work within the constraints of a real-time environment and keep track of various options, which may be either static or dynamic. Plans are executed only while the appropriate conditions are maintained. Once the conditions change, the situation is re-evaluated. Should a new goal or event arise, the agent is able to prioritize and multi-task as required. These characteristics illustrate the key advantages over realtime expert systems.

\subsubsection{Capabilities}

Similar to the way that subroutines can be grouped together in single blocks on a highlevel diagram, capabilities are a means of clustering the common reasoning elements of agents together. This is a technique which simplifies the agent system design and allows for the reuse of code. Capabilities also have similar functionalities to agents and are able to share the same plans. As the design evolves, it may be necessary to use capabilities to simplify code development and remove duplication. In this author's experience, it is easier to design a preliminary system and then augment the architecture with capabilities as required. 


\subsubsection{Events}

Within JACK, events are the action items that guide an agent. Events lie at the origin of all activity within an agent-oriented system. Essentially there are three different types of events: internal stimuli, external stimuli and motivations.

Internal Stimuli are events that an agent sends to itself as a result of executing reasoning methods in its plans. These internal events form an integral part of the ongoing execution within an agent and the reasoning that it undertakes.

External Stimuli could include messages from other agents, or percepts that an agent receives from its environment.

Motivations of an agent can include goals that the agent is committed to achieving.

Furthermore, there are two broad types of events, normal events and BDI (belief desire and intention) events. A normal event initiates a response from the recipient agent to take immediate action, whereas a BDI event moves away from plan-directed behaviour into goal-directed behaviour. This is a subtle but very important difference. BDI events force an agent to strive towards a desired long-term goal, rather than defining a particular plan of action. Furthermore, these events supplement information in the decision-making ability of the agent, and may influence the outcome of this process. A BDI event includes meta-level reasoning (or reasoning about reasoning) for; plan selection, a course of action in the event of a plan failure, and the ability to perform recalculations in the event of a plan failure - which omits the involvement of this failed plan. Comparing the two types of events it can be seen that normal events cause an agent to pick the first 
applicable plan once it checks that the chosen plan is relevant under the circumstances. BDI events on the other hand allow an agent to assemble a set of plans in order to achieve its goal. The latter is more powerful and complex because it is able to influence the behaviour of the agent, especially when a plan is unsuccessful.

\section{$\underline{\text { 4.1.4 Plans }}$}

Plans are the pages of the procedure manual, which are used by the agent once it receives an event. Within each plan is a basic set of conditions that the agent uses to determine the relevance of a particular plan to the given situation. Once selected, the various phases of the plan must either succeed or fail. If the plan should fail for any reason, or if the reasoning method, which can be used to call other plans, should fail, the agent is notified.

\subsubsection{Named Data}

Named data are the databases from which a plan may draw information. During the execution of the system, it may be necessary to update the database.

\subsection{Design Methodologies}

One of the challenges currently facing the agent programming community is the ad hoc fashion in which multi-agent programs are being developed. While there are many methodologies, none are followed rigorously. The European Network of Excellence recently prepared an agent roadmap for Agent-Based Computing (Luck et al., 2003). The report identifies the need for a method which can model the systems with sufficient detail without concern for implementation details. Such methods would provide the basis for 
verification, validation and testing of system properties in light of specified requirements, and within these specifications the system properties such as scalability, performance, reliability and robustness can be addressed. The Prometheus design methodology of Padgham and Winikoff (2004) addresses these issues, and it is this methodology on which JACK intelligent agents have been based. JACK provides the framework for developing agent-oriented programs, just as Visual Basic does for object-oriented programming. In June 2004, Wooldridge described the Prometheus design methodology as one of the most mature methodologies used today.

The Prometheus design methodology consists of three phases of processes for specifying, designing, and implementing agent-oriented software systems. The methodology adopted from Padgham \& Winikoff (2004) is shown in Figure 4-1. This same figure is referred to again in Chapter 6 where it is used to develop the agent program in this thesis.

There are three phases within this waterfall design methodology. The first phase focuses on identifying the goals and basic functionalities of the system, along with inputs (percepts) and outputs (actions). The second phase uses the outputs from the previous phase to determine which types of agents are required, and how the agents will interact. The final phase looks at the internal components of each agent and explores how the agent will work to accomplish its task within the overall system. The methodology does not address program testing, fault tolerance analyses or coverage profiling. 


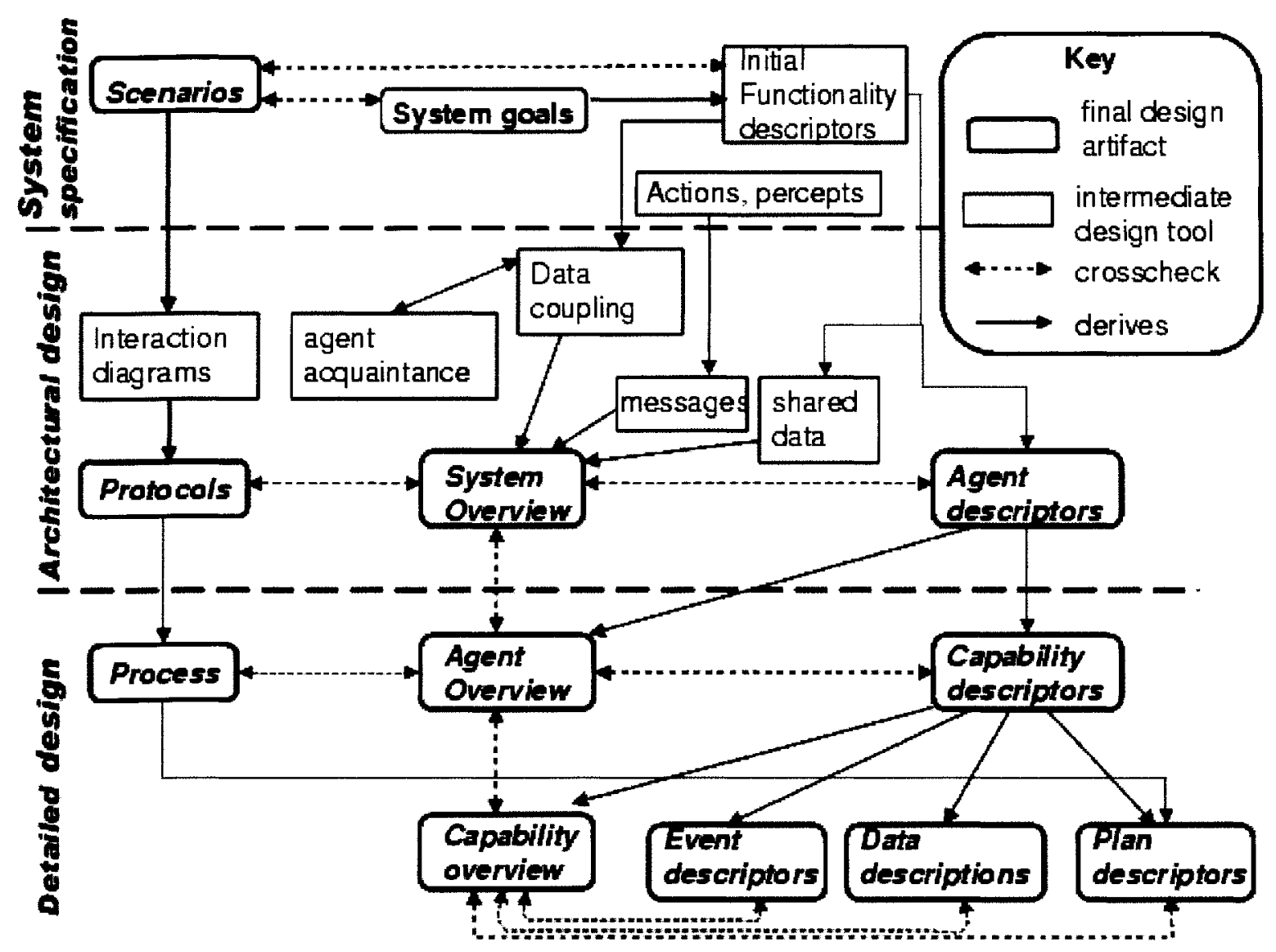

Figure 4-1: Prometheus methodology overview

In addition to designing the methodology, Padgham and Winikoff have also created a software version of the Prometheus design tool that guides the programmer through each step. While this software tool provides a useful experience for understanding how multiagent programs can be developed, the resulting skeleton code generated by the software Prometheus program is not currently transferable to the JACK environment. Future work may involve enabling the results to be imported into JACK, however a lack of funding and time has prevented this from happening to date (L. Padgham, personal communication, 30 May 2004). As such, the Prometheus design methodology is used in conjunction design tools found within the JACK development environment. 
The graphical design environment of JACK permits the programmer to develop a detailed picture of the program by pulling elements from the design palette, and placing them onto the design canvas. Links between the various elements illustrate the inter-relationships. As the graphical design is created, JACK automatically generates a skeleton code structure. Relationships between and among components are included in this skeleton code. It is worthy to note that teams can also be generated in JACK; however, for a single air vehicle, this feature is not used and so will not be elaborated here.

Other agent-oriented programming methodologies exist, and include Gaia (Wooldridge et al., 2000), Tropos (Bresciani et al., 2002; Giunchiglia et al., 2002), MaSE (DeLoach et al., 2001), and PASSI (Burrafato and Cossentino, 2002; Cossentino and Potts, 2002).

\subsection{Inter-Agent Communication}

\subsubsection{External Communications}

JACK uses the user datagram protocol (UDP) when communicating between agents which are not located within the same instance of the software. Through UDP, it is not possible to confirm that all the data within one packet were received; therefore, JACK wraps an extra layer or mediator to the message, which is read and understood by the recipient agent. While UDP does not contain built-in checks, both Transmission Control Protocol (TCP) and Space Communications Protocol Specification Transfer Protocol (SCPS-TP) include this feature. TCP enables two computers, each with unique network addresses to establish a connection and exchange streams of data. TCP guarantees delivery of data and also guarantees that packets will be delivered in the same order in 
which they were sent. Within the data protocol hierarchy, SCPS sits in the layer above TCP. It is typically used in spacecraft communications environments, characterized by potentially long delays, unbalanced forward- and return-link data rates, and potentially high error rates (SCPS, 1999). Given the environment of UAV operations, it is desirable to use the SCPS-TP between the GCS and autopilot system. Under the current test configuration, as described in Section 7.1, UDP packets of information can be passed over a regular Local Area Network such as that contained within the UAV, and with some modifications could be integrated with TCP or SCPS-TP, as required.

\subsubsection{Internal Communications}

When multiple agents must interact, mechanisms need to be established to handle the coordination of the communications. There are three primary methods to handle cooperation: (a) centralized allocation of tasks by trader, (b) distributed allocation of tasks, and (c) emergent allocation. Figure 4-2 adopted from Ferber (1999) shows the relationships among the various methods.

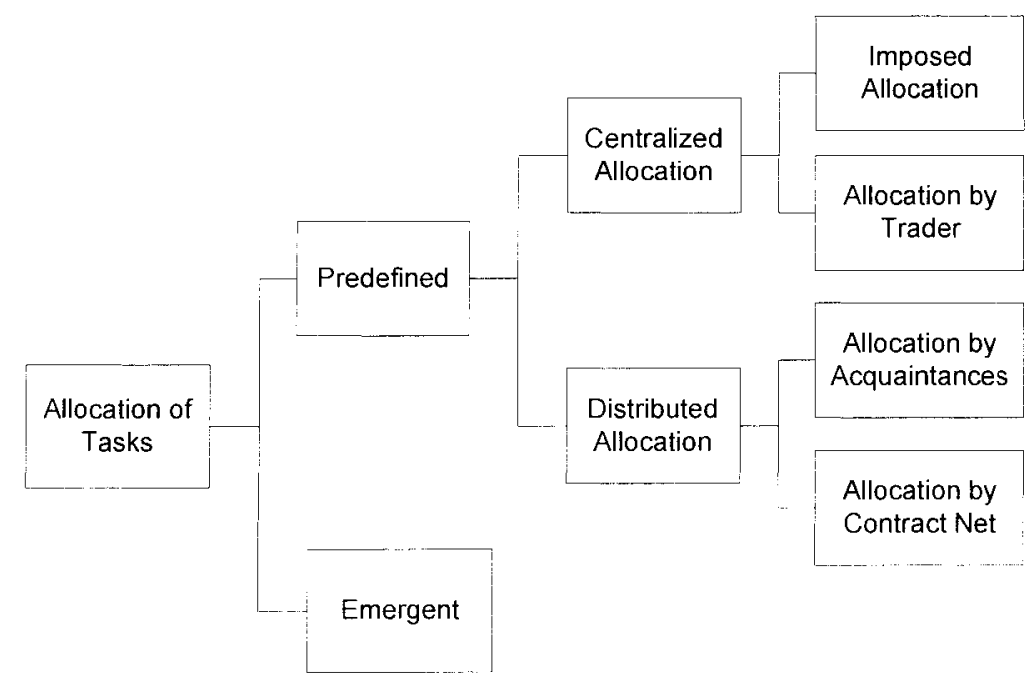

Figure 4-2: Principal tasks distribution modes 
a) Centralized Allocation: Under this regime, when an agent needs to carry out a task, but for some reason it is unable to do so, it requests help from a central trader. This trader sends out a request to the other "supplier" agents that are known to have the necessary capability. If a supplier responds to the trader's request, the client is informed in an acknowledgment that is sent to the supplier from the trader. This centralized approach has both advantages and disadvantages. As long as the trader agent is aware of the suppliers' capabilities, then this system is effective in determining an optimized solution. This setup ensures that the best agent is working on the solution. However there are two major drawback of this solution. The trader agent must handle a considerable number of messages at any given time. This effectively degrades the performance of the trader and is prone to creating a bottleneck in the system. As the number of agents within the system increases, the number of exchanges between agents and traders grows exponentially; Ferber (1999) notes that a system which includes 50 agents, requires the trader to process 500 messages, while a system with 100 agents increases the number of messages to 2000 . The second drawback to this system is the fragility of the trader. If for some reason the trader was to go down, or leaves the organization, then the entire system falls apart. While it is possible to have multiple traders, this raises additional coordination problems when they are all functioning properly. For a distributed system of agents within a UAV system, multiple points of failure already exist, and this centralized allocation method could crash the entire system. It is for this reason that this author does not advocate using the centralized allocation method. 
b) Distributed Allocation: This section provides an overview of the distributed allocation method, which is further highlighted through the description of allocation by the contractor net that was first proposed by R.G. Smith in 1979. Both allocation by acquaintances and allocation by contract net provide a market-like protocol for task allocations. Here a system includes a manager and a number of bidders, similar to a system that includes multiple agents. The protocol outlines the communication exchanges that occur during a request for bids phase of a project. With the contract net method, the first of the four steps has the manager draft a description of the problem and determine which agents within the system should manage the task. After reviewing the description, the bidders send proposals to the manager. The manager reviews the proposals and awards the contract to the best bidder, considering various criteria such as quality of proposal, credibility, capacity of bidder, cost, among others. The final stage requires the winning bidder to confirm that they are still in a position to carry out the contract. On the subject of contracting nets, Ferber (1999) notes that while contract nets offer great flexibility to the manner in which agreements between clients and suppliers are managed, it is not a model for the resolution of problems. The benefits of contract nets are that they are simple to create and have been designed to operate within a distributed architecture. Furthermore, contract nets can be very dynamic, allowing new agents to check-in or check-out of the process depending on their availability. While the general design of the contractor net is simple, the greatest drawback of this proposal is that it is extremely difficult to implement. It works well for high-level tasks, which involve only a few agents. In fact, the number of messages, $M$, for such a net is given by 
$M=N+2 \alpha N$

where $\alpha$ represents the ratio of the number of bidders interested in a request for bids, to the total number of agents, $\mathbf{N}$, in the system.

Negotiation mechanisms are required when multiple agents try to communicate. The contracting net is used when different agents may be bidding on the ability to complete a certain task, but depending on the nature of the problem, the final task may require the abilities of more than one agent. Therefore, a full description of the contract net is required at the outset.

Having now established a general understanding of intelligent agents and multi-agent systems, Chapter 5 describes the hardware and software systems found within a UAV with which the agent-based programme must interface. 


\section{Chapter 5: UAV System}

\section{Overview}

\section{CHAPTER SUMmary}

When designing a complete UAV system, the process involves understanding how the system-of-systems work together. This chapter provides an introduction to each of the individual components including; ground control station and aerial vehicle system (payload, autopilot, and target recognition algorithm). Understanding the interfacing needs of each system is crucial to making everything work together. 


\subsection{UAV System Overview}

Having established what is involved in multi-agent programming, this section examines the 'system of systems' aspect of UAVs. Although no specific system has been defined, a notional system that is suitable for the demonstration mission is described in this chapter. Concurrently, the basic requirements for the system components are also defined.

\subsection{UAV Subsystems}

A UAV system consists of two primary subsystems, the ground control station and the air vehicle. This section provides an overview of each subsystem.

\subsubsection{Ground Control Station}

All interactions between the human operator and the air vehicle must go through the Ground Control Station (GCS). A generic ground control station has seven primary functions as shown in Figure 5-1. Missions are planned using maps, terrain and weather information from external sources (3). As part of training (2c), the missions are often planned and simulated first before any information is transmitted (4) to the air vehicle. During the execution of a mission, all air vehicle information is displayed to a ground operator through a human computer interface (HCI) (1) and through a payload display (5). 


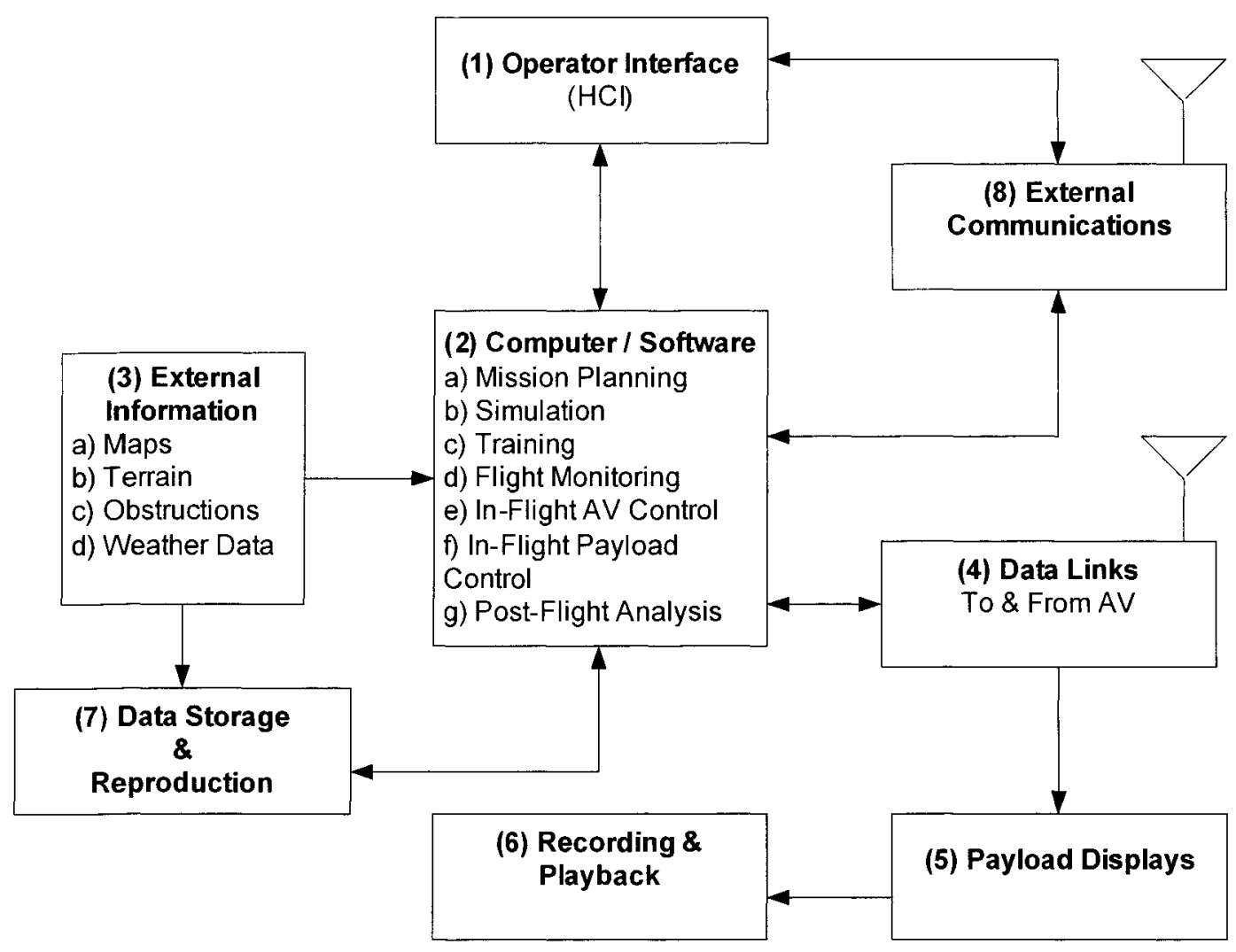

Figure 5-1: General ground control station overview (Carryer, 2004)

\subsubsection{GCS Operations}

Often within today's operations, a human operator takes an active role in controlling the UAV and its camera system. However, research groups within NATO have made it very clear that this approach to manually flying the UAV and controlling the sensor is less than ideal (personal communication, P. Farrell, CFEC, 16 March 2005). Ideally, an operator's only tasks involve defining the search area and transmitting a waypoint file to the UAV. Once the waypoint file has been uploaded to the UAV, the vehicle should execute the search pattern, identify possible targets, and transmit the images that are obtained. During a SAR mission it will be the responsibility of the GCS operator to examine the images of possible targets and to inform the local searchmaster if a target 
warrants sending a team of SAR technicians and a helicopter to the specific location to gather more detailed information.

\subsubsection{Mission Planning Aids and Operator Interfaces}

Information used during the mission-planning phase requires initial input from the operator through the human computer interface and includes external information from local databases. Once the initial search area has been identified and a series of flight lines have been drawn over the area, a series of waypoints are generated, where a single waypoint includes a latitude, longitude and altitude. Once the waypoint file has been generated, it is sent to the UAV, where the file is stored in a local database.

Ruff et al. (2002) found that for the control of a single vehicle, mission efficiency was highest where the operators were kept in the loop. Under their test environment, three levels of control were possible:

1. Manual (automation is dormant until initiated by an operator)

2. Management-by-consent (automation proposes action but cannot act without explicit operator consent)

3. Management-by-exception (automation acts without explicit operator consent), requiring specific commands from the operator to cancel automation.

As the complexity of Ruff's mission increased along with the number of vehicles being monitored by a single operator, the mission efficiency increased with management-by- 
exception; however, the operators noted that they had lost situational awareness because they were not aware of the status within or around the air vehicles. While the study performed by Ruff et al. was based on a military scenario, it reinforces one of the original airspace integration concerns, expressed by Capt. J. Bernier cited in Chapter 3. That is, when it comes to operating a UAV in the same airspace as a manned search and rescue aircraft, it is imperative that the GCS operator knows the situation and environment of the UAVs at all times.

\subsubsection{Decision aids}

The functions of decision aids are standardized, whether they are used within a manned aircraft to aid the pilot, or used within a UAV to aid a ground operator. The Defence Evaluation and Research Agency (DERA) of the UK stated that the use of intelligent decision aids should ensure that a pilot could focus his attention towards the relevant critical mission events, at the appropriate time, at the appropriate level. DERA's cognitive cockpit project was conducted over a three year period through The Technical Cooperation Program (TTCP) HUM TP-7. Considering that the GCS is simply the cockpit of the unmanned aerial vehicle on the ground, then it is reasonable to consider the work of Taylor et al. (2002), which suggests, that the requirements of intelligent aiding systems within the cognitive cockpit should include the cognitive versatility, the management of integration and complexity, while performing in real-time. Each of these concepts will be discussed in the following Section. 


\subsection{Cognitive Versatility}

Any intelligent aid system should be capable of performing concurrent reasoning, which is consistent with the distributed artificial intelligence structure of JACK agents. Furthermore, the system should include a number of different methods of reasoning, including incremental reasoning, in order to produce the most accurate assessment of the current situation. This suggests that there is a need to share information between individual agents before a decision is made. In all cases it is imperative that the system is able to describe all aspects of its behaviour to the operator.

\subsection{Management of Integration}

Not only must an intelligent aid system be able to investigate targets of interest, but it must also perform routine checks-and-balances over an extended period of time. By having a number of distributed agents within the air vehicle system it is possible to not only flag anomalous behaviour but also to perform on-board diagnostics.

\subsection{Management of Complexity}

With numerous agents exchanging information, it is possible to overwhelm the system of agents with data. Since some decisions must be made in real-time, selecting which data to process requires the system of agents to identify priorities. Furthermore, the system must be able to identify time-critical data while continuing to perform routine functions, all the while dealing with new information. Failure to do so may result in the air vehicle falling out of the sky. Limiting the scope of responsibility for any given agent is necessary to 
ensure that the system can achieve specific goals, while identifying and solving the most urgent problems first.

\subsection{Real-Time Performance}

Latency is a reality within any system; however, measures must be taken to ensure that the tasks are still performed within the necessary time frame. As the task levels increase, putting more pressure on system resources, the response latency of the system must decrease. Doing so may compromise precision and confidence but this may be acceptable if it is done in a graduated manner.

\section{$\underline{5.1 .2 \text { Aerial Vehicle Svstems }}$}

Without regard for the specific airframe, an air vehicle system contains propulsion, avionics, payload and data link elements. Seamless communication among all elements shown in Figure 5-2 is possible through a TCP/IP network located within the air vehicle.
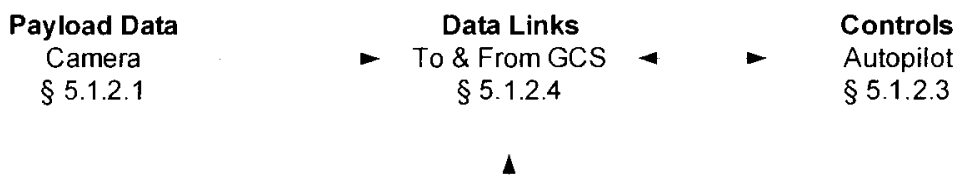

$$
\begin{gathered}
\text { AV Status } \\
\text { ATR } \\
\S 5.1 .2 .2
\end{gathered}
$$

Figure 5-2: Air Vehicle configuration (Carryer, 2004) 
In the studied SAR application, the sole payload sensor onboard the air vehicle is a fixed position high-resolution camera (Section 5.1.2.1). An automatic target recognition algorithm (ATR) processes the image (Section 5.1.2.2). Depending on the output from the ATR, the UAV is controlled through the autopilot system (Section 5.1.2.3). Data transmissions between the air vehicle and the ground control station (GCS) require the passage of information through the data links (Section 5.1.2.4).

\section{$\underline{\text { 5.1.2.1 Camera }}$}

There are three primary objectives of the image acquisition system:

1. To provide complete imagery coverage for the search area.

2. To acquire images that can be geo-referenced.

3. To provide sufficient "pixels on the target" to find and identify the missing aircraft in a variety of different terrain environments.

Various configurations of the camera installation were initially considered. These included positioning the camera at oblique angles on either the port or starboard side of the UAV, or forward looking. A second alternative considered a gimballed system, which would scan the terrain as the UAV flew overhead, similar to a manned SAR platform where technicians (spotters) are located on both sides of the aircraft, and use their eyes to progressively scan the landscape (Chief of Defence Staff, 1982). To reduce eye fatigue, spotters generally work in shifts, where a $\mathrm{CH}-146$ utilizes two spotters, a CH-149 carries four spotters, and a CC-130 carries six to eight spotters (Bernier, 2004). 
In order to ensure complete coverage by the camera, and to acquire images that could be easily geo-referenced, it was decided that the camera would have zero-degrees of freedom and would be positioned perpendicular to the flight line. This orientation makes system calibration much simpler than a pan-tilt or pan-roll, two degree of freedom camera. By knowing the position for the camera's position relative to the position for the GPS receiver, it is possible to know the position of the camera's footprint, as compared to the global reference frame, see Figure 5-3. Through differential GPS techniques, it is possible to provide sub-metre positional accuracies for the UAV depending on it's location in the world. However the positional accuracy of the geo-located of ground targets is not as accurate. 


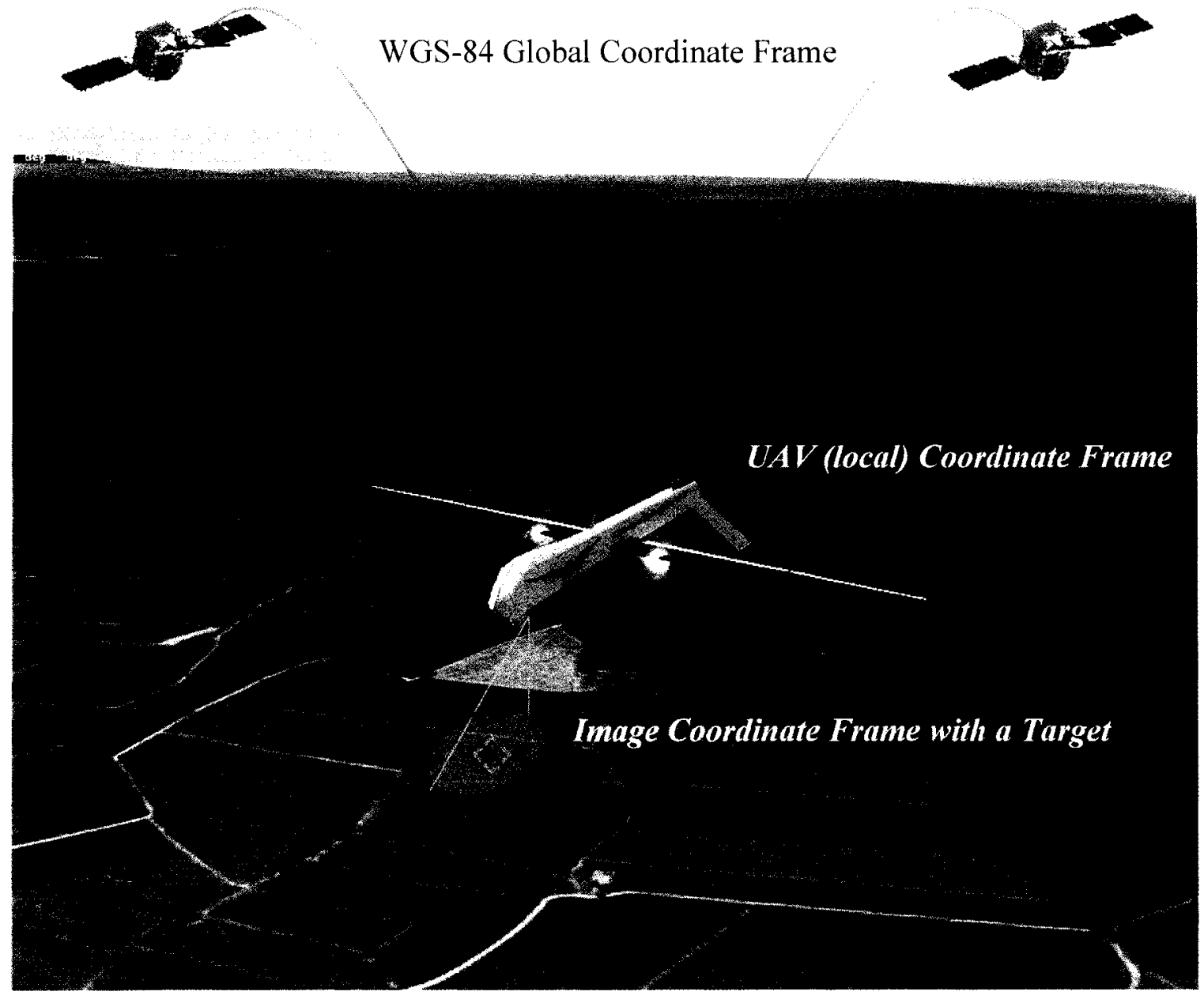

Figure 5-3: Target geo-positioning through coordinate frame translations

To gain a better appreciation for the appearance of wreckage spotted from altitude, the author consulted with SAR Technicians at JRCC Trenton. Based on personal communications with Sgt. J. Leclerc (18 Nov 2004), typical aircraft wreckage generally consists of large pieces. The fuselage is usually intact, however it is often "ripped open, crumpled, dented, and possibly charred from fire. But usually it remains in one piece". Sgt. Leclerc also mentioned that the tail section generally survives most accidents, and ends up pointing to the sky. Wings are usually visible if they are laying flat. However, if the search object crashes at a high angle of attack, as is often the case when an aircraft 
stalls before impact, there are usually only very small pieces left intact. Post-crash fires generally reduce the wreckage even further, and wings are often charred, though they do tend to remain intact.

Therefore, the imaging system must be capable of capturing whatever remains of the search object, permitting the automatic target recognition algorithm as described in Section 5.1.2.2 to identify the object. For the purposes of this work an appropriate camera may be a commercially available, 5 Mega-pixel Canon digital camera, with a standard field of view equal to one radian $\left(\sim 57^{\circ}\right)$. Therefore, the height flown above the ground will be equal to the swath width. At an altitude of 300 metres, the camera provides a resolution of $0.02 \mathrm{~m}^{2}$ per pixel. Assuming the $6.8 \mathrm{~m}$ long fuselage of the Piper Super Cub remains intact, this yields approximately 340 pixels on target. The importance of this number is discussed in the following section.

\subsubsection{Automatic Target Recognition (ATR)}

The ATR algorithm performs three functions;

1. The detection of possible targets,

2. The identification of potential targets, and assignment of a confidence value, and

3. The geo-location of potential targets.

The automatic target recognition algorithm is currently under development. Within the search and rescue mission, a number of photographs of actual incidents were obtained from JRCC Trenton to train the ATR. Because the targets are located in complex background clutter rather than in open fields, a number of training exemplars are 
required. The algorithm must be taught what should be included in the target class, and counter-trained to populate non-target class.

In 1951 the US military developed the concept of line pairs per millimetre, as a unit for measuring the resolution of image intensifiers. Using a series of different sized patterns, which were composed of three horizontal and three vertical lines, the "air force resolving power test target" was created. In the early work of Johnson (1958), identification of a target was based on the resolution of line pairs on a target's minimum dimension. For example, the minimum dimension of a Jeep is its height. A minimum of 1.2 line pairs was required to detect the Jeep, while 5.5 line pairs were required for identification. While today's night vision goggles still measure their resolution in line pairs per millimetre, the ATR algorithm uses a digital image as its source, and now works on the basis of clustered pixels on target.

Based on current work, the ATR has been trained to identify military vehicles from a UAV platform. Rather than specifying a minimum number of pixels along the principal axis of the vehicle, the minimum requirement is stated as the number of pixels on target. This distinction eliminates concerns about target orientation. At a minimum, 1 or 2 pixels clustered on target are required for detection, while identification requires a minimum of 200 pixels on target. Recall that while flying at $300 \mathrm{~m}$, the fuselage of a Piper Cub would be 340 pixels. 
As with any automated decision-making system, errors are likely to occur. For this reason, the ATR algorithm also includes a confidence number between 0 and 1 for each possible target. By using a ROC curve that represents the probability of detection vs. the probability of a false detection (POD/POF), the sensitivity of the ATR and sensor system can be varied, as shown in Figure 5-4. This will permit future tests to include different sensor models without affecting the rest of the overall UAV system.

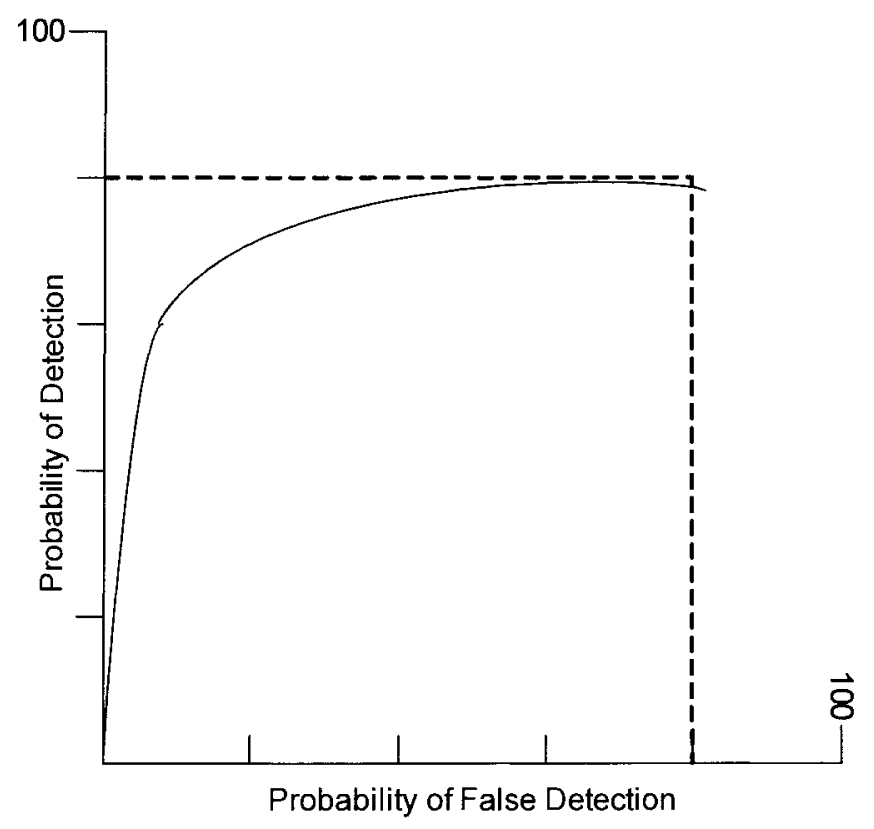

Figure 5-4: POD v. POF curve will be sensor based

One way to ensure that a target will be found is to also accept numerous false detections. As such, the algorithm will be sensitive to all anomalies. The ground operator may wish to accept a different threshold for the number of acceptable false positives depending on the characteristics of the region being searched. 


\subsubsection{Autopilot}

The autopilot system is capable of controlling the UAV by sending signals to the control surface actuators. Currently, autopilot systems are used in one of two ways; either the vehicle is provided with a set of waypoints and is instructed to reach these points, or a ground operator flies the vehicle manually. In either case, the onboard autopilot remains in communication with the ground control station. When used with intelligent agents, the autopilot must support the agents with information obtained by internal navigation aids such as accelerometres, and from airspeed information derived from the pitot static probe.

The MicroPilot MP2028 autopilot system was chosen as a reference for this project because this system is currently in use at Carleton University. Inputs to the MP2028 include waypoint information (either relative to the starting position in metres, or in latitude and longitude format), desired altitude, and desired airspeed. As with many UAV autopilot systems, a maximum bank, dive, and climb angle of 20 degrees is possible. Based on personal communications with P. Chambers of Micropilot (15 Nov 2004), the MP2028 autopilot reads one waypoint at a time. This information is relevant to future discussions in Chapter 6 about route planning by the agent software. As such, it does not matter whether an entire mission of waypoints is transmitted to the autopilot, or if the waypoints are sent individually. The autopilot is not aware of the next waypoint until it reaches the current target waypoint. 


\subsubsection{Data links}

A data link, whether it is through line of sight or over a satellite communication link, connects the air vehicle and the ground control station. During line-of-sight operations, it is possible to transmit video imagery and vehicle health information at approximately 80 kbps using a Link-11 or Link-16 radio system. However, once beyond line-of-sight the data stream is generally limited to $2-3 \mathrm{kbps}$ using commercially available satellite systems. While there are compression methods that can be used to increase the available bandwidth, these data rates become the limiting factor when it comes to passing large amounts of data (video, images, air vehicle states) between the air vehicle and ground station. 


\section{Chapter 6: Programme}

\section{Development}

\section{CHAPTER SUMMARY}

This chapter describes the development of the multi-agent programme. Using the initial building blocks given by Chapters 3-5 this chapter proposes a location for the JACK program within an air vehicle's internal architecture. Following the system engineering approach, the requirements and functional analyses are performed by following the Prometheus design methodology (Padgham \& Winikoff, 2004) for multi-agent systems. The methodology involves many steps where the results following each step are described in this chapter. Both a top-down approach of defining high-level goals and a bottom-up approach of defining each interface is used in the development of the software program. This chapter also provides the reader with a description of all design decisions, including the three primary algorithms which are invoked to control the UAV depending on target confidence values and positions that are provided by the automatic target recognition algorithm. 


\subsection{Programme Development}

Using the system design process, described by Figure 6-1 as adapted from the Systems Engineering Fundamentals Guide (Department of Defence, 2001), the first step involves understanding the process inputs. In previous chapters these inputs have been described:

- Chapter 3 described the SAR mission and environment,

- Chapter 4 provided details of the software technologies, and

- Chapter 5 gave a description of the basic hardware technologies

Process Inputs

- Customer Needs/ Objectives

Requirements

- Missions

- Measures of

effectiveness

- Environments

- Technology Base

- Program Decision

Requirements

- Requirements applied

through specifications

and standards
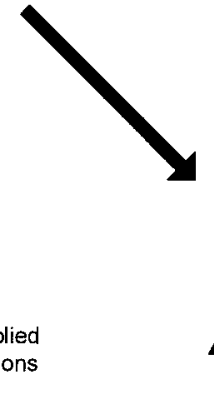

Requirements Analysis

- Analyze missions and

environments

- Identify functional requirements

- Define performance and design

constraint requirements

$\Delta$

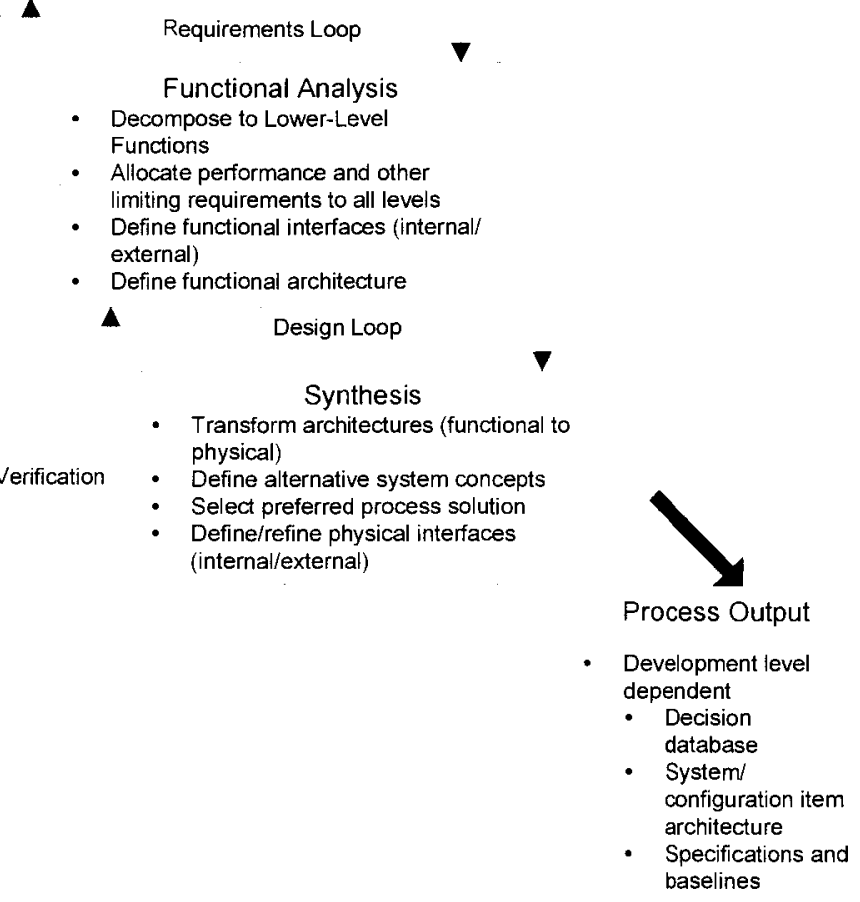

Figure 6-1: The systems engineering process 
By combining the information provided in Chapters 3-5, a notional air vehicle system is developed in Section 6.1, which also includes intelligent agents. Section 6.2 describes the multi-agent software design that was developed through an iterative process of requirements and functional analyses prior to synthesis using the Prometheus design model. Section 6.3 examines the implications of using intelligent agents to the design standards and interfaces.

\subsection{Intelligent Agents within the Air Vehicle \& GCS}

Central to the integration of JACK within an air vehicle system is Figure 6-2, which identifies the proposed flow of data, commands and perceptions from the environment and the major components onboard the aerial vehicle.

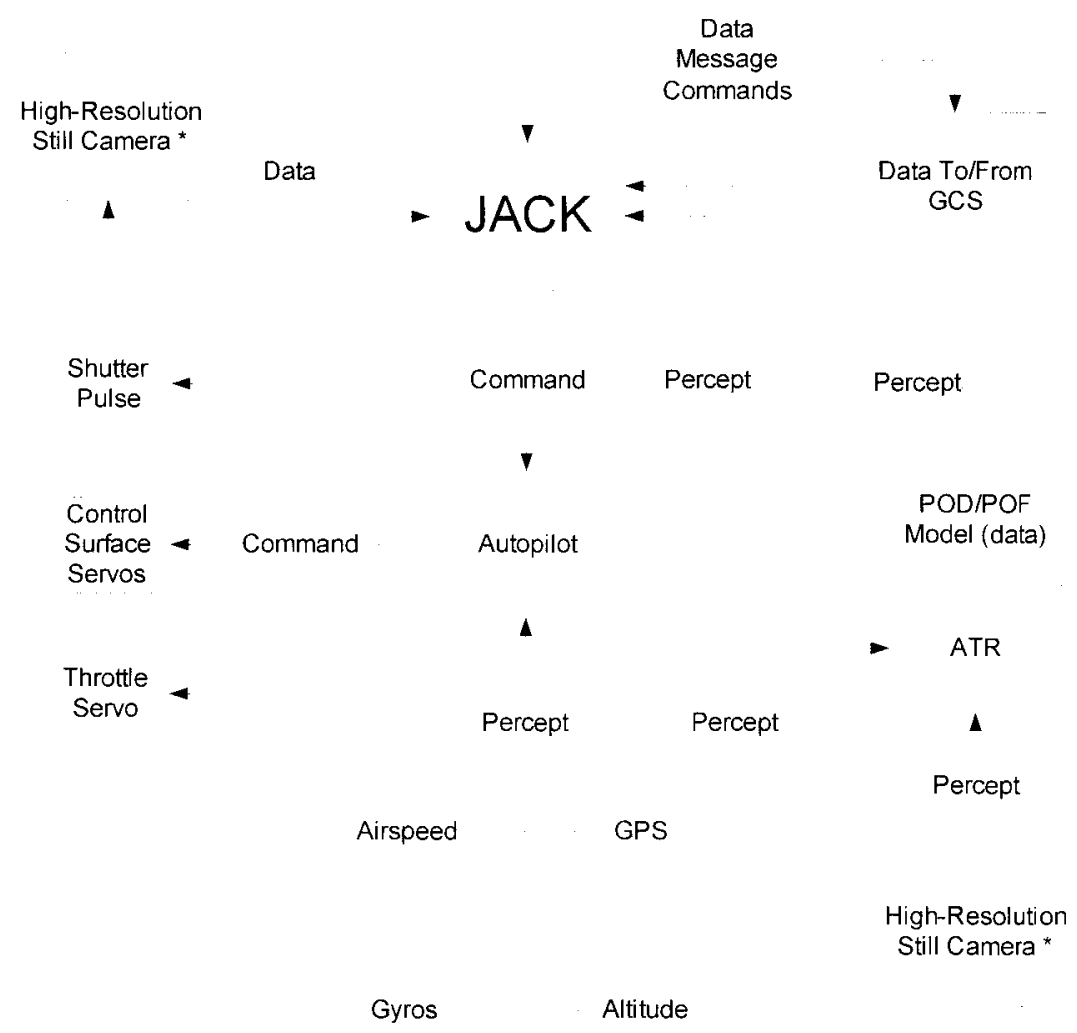

Figure 6-2: Percepts, commands and data flows within the UAV 
All external data gathered by the UAV's sub-systems are shown as a 'percepts'. As shown, the autopilot has four such percepts. Similarly, the ATR requires that an image is first acquired using the high-resolution still camera, before it can be processed. The output from the algorithm is a confidence value that is coupled with the latitude and longitude of each possible target. These values are then passed to the intelligent agent software (JACK). Depending on the confidence value, different courses of action may be taken. In order to control and monitor the progress of the vehicle, the intelligent agents communicate with the autopilot system, which in turn controls the air vehicle and reports on the current vehicle status (airspeed, altitude, position). High-resolution images are acquired when the air vehicle flies over the target of interest. Once the image is acquired and transmitted, final interpretation is the responsibility of a human operator or image analyst at the ground control station.

\subsection{Programme Development}

Continuing with the system engineering process the previously mentioned Prometheus model for project development is used. Through this process, this section describes requirements, examines the functions that can be performed by individual agents and describes the design and development of the system architecture. As shown in Figure 6-4 Section 6.2.1 addresses the system specification phase, Section 6.2.2 the architectural design phase and Section 6.2.3 the detailed design phase. To ensure traceability, the rationale for all design decisions is also included. 


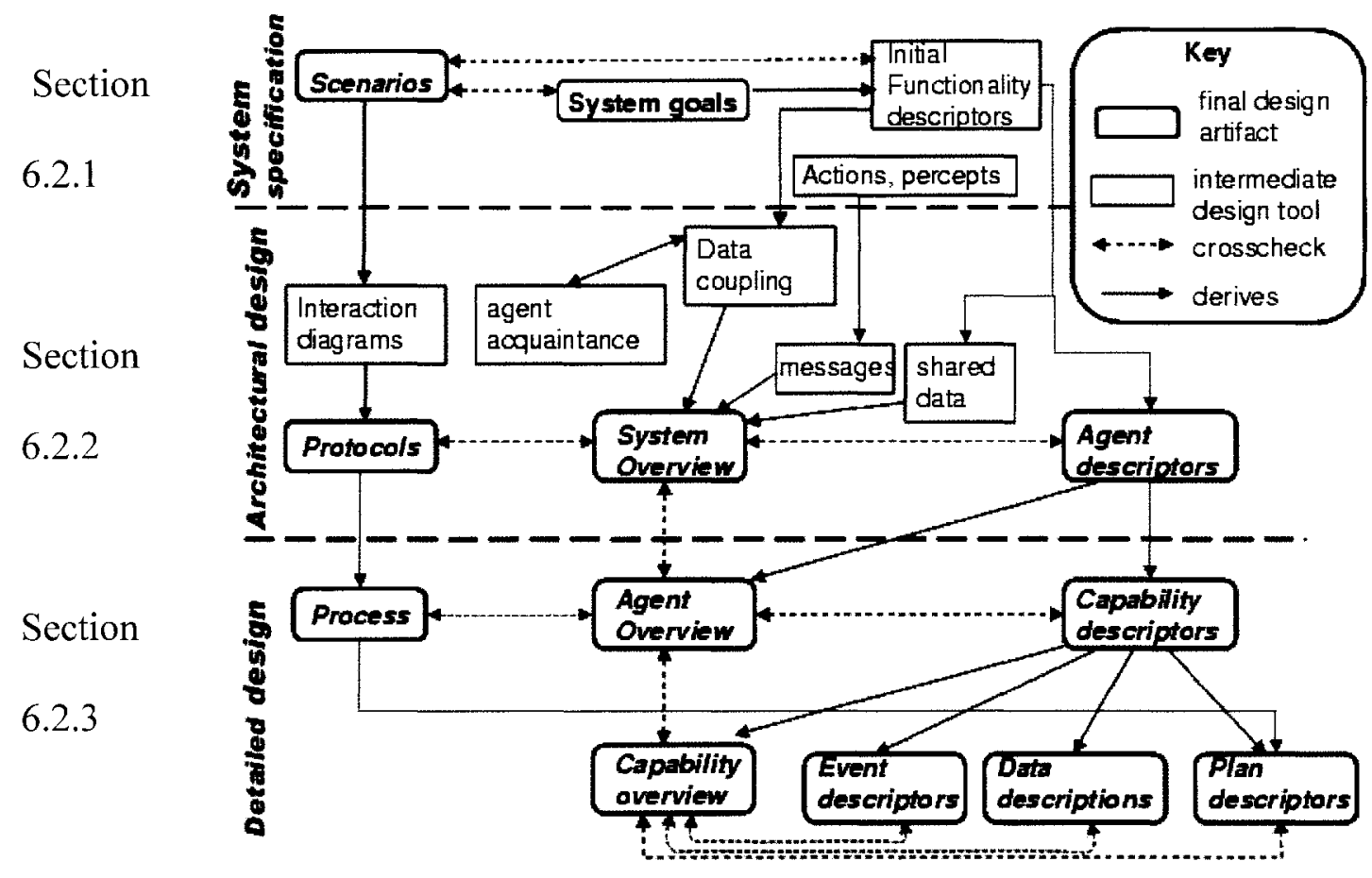

Figure 6-3: Prometheus model for multi-agent programme development

\subsubsection{System Specification}

Two methods are available for system specification. A top-down approach initially uses goal development, while a bottom-up design begins by identifying the agents and their responsibilities. While each method is valid if used individually, a mixture of top-down and bottom-up design is both common and advantageous in agent-oriented systems. In this thesis the top-down approach is described in Section 6.2.2.1, followed by the results of the bottom-up approach in Section 6.2.2.2.

\subsubsection{Goal Development and Refinement}

Independent of which method is chosen, goals are central to the development of all agentoriented programming. Setting high-level goals provides a starting point for building an 
initial set of system goals. These goals provide an overview of system expectations, and facilitate the development of requirements. After determining the high-level goals, refinements are necessary to identify sub-goals. These sub-goals are developed by asking, 'how can the goal be achieved?' Both high-level and derived sub-goals for this research are described in Figure 6-4.

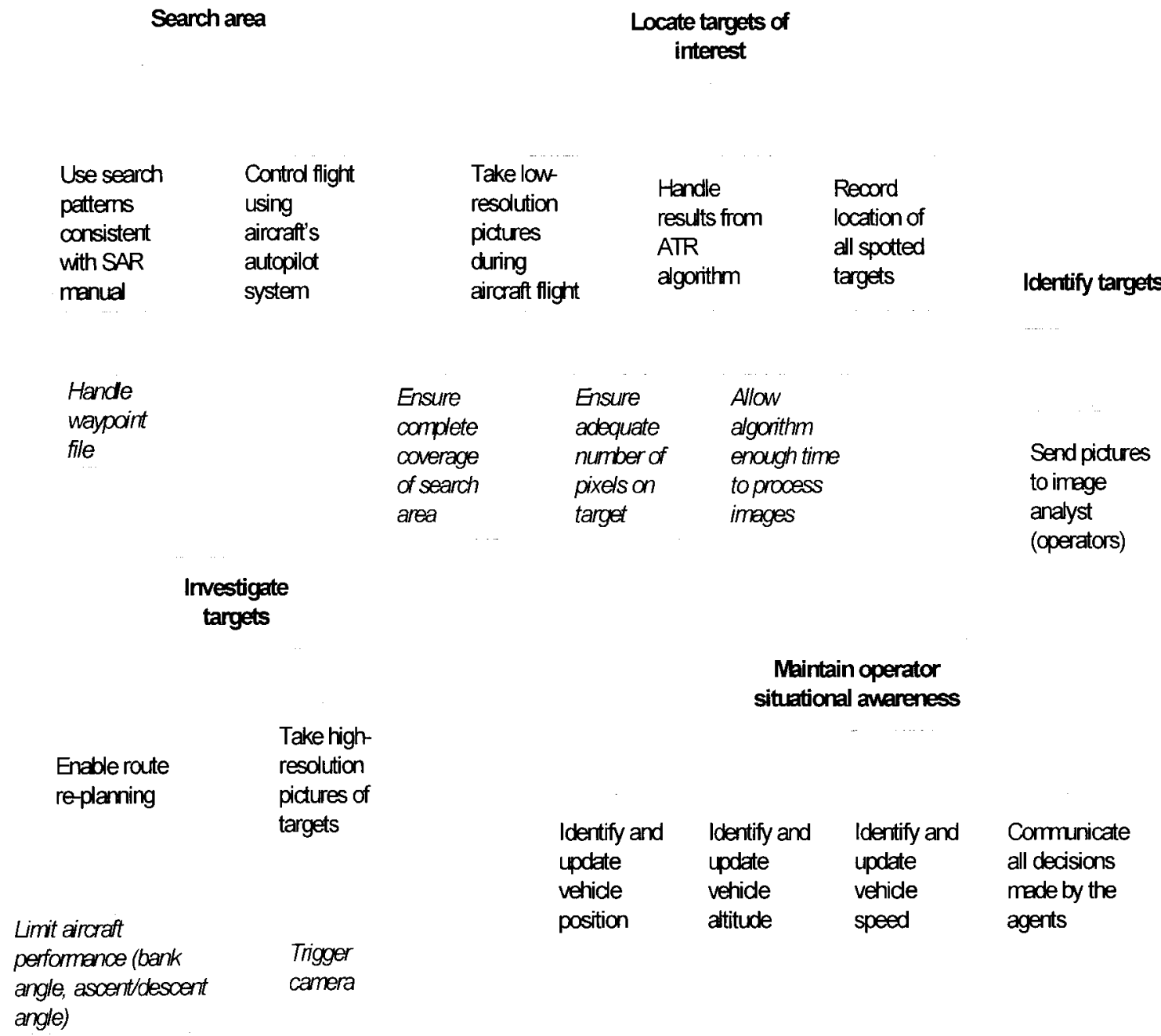

Figure 6-4: Goal development

One of the high-level goals is to perform a complete search of any given area. In order to accomplish this high-level goal, two sub-goals were developed. The first sub-goal says 
that in order to perform the complete search, the UAV must follow one of the search patterns defined in Appendix A. The second sub-goal states that in order to fly the UAV, commands must be sent by the autopilot system.

\subsubsection{Scenario Development}

Three different scenarios exist within the SAR-based search and identify mission. Each scenario is based on the aircraft performing a search, including flying a series of latitude and longitude points, at an altitude of $300 \mathrm{~m}$ above ground level (AGL). The different scenarios depend on the detection of targets by way of the ATR algorithm, which provides target locations and confidence data.

Closer Look: Upon determination of a high-probability target, (confidence value $>0.8$ ) the ATR is turned off. The vehicle circles and descends to $150 \mathrm{~m}$ AGL, lines up on the coordinates of the target and takes a minimum of one high-resolution image over the target. The image is then transmitted to the GCS while the UAV returns to the original flight altitude and track line. The ATR data is re-engaged once the UAV is at the same latitude, longitude, and altitude that it was turned off.

Optimized Pass: All medium-probability targets (confidence values between 0.4 and 0.8 ) are flagged while flying at $300 \mathrm{~m}$ AGL. At the time of spotting, the position, target confidence value and heading of the aircraft are recorded. After completing the entire search pattern, the UAV descends to 150 metres AGL and loiters while an operator 
selects the desired order to revisit the flagged targets. A high-resolution image is obtained for each target location.

General Overview: For any low-probability targets (confidence values $<0.4$ ) seen from 300 metres AGL, their position and associated target confidence value are recorded and stored in a local database. After completing the initial search, the search pattern is rotated $90^{\circ}$ and the search is performed again while still at 300 metres. Confidence factors of targets at previously identified locations are compared and ignored if any target has been previously photographed. As with the optimized pass, medium-probability targets should have their position flagged and high-probability targets must be investigated immediately.

\subsubsection{Architectural Design}

\subsubsection{Top-Down Approach}

During the top-down development process, the first step is to identify what functionalities must be performed by the multi-agent system. Following this analysis, a description is provided of the individual perceptions and actions that are required. Grouping similar functionalities is necessary before discussing the organization and number of agents required within for the final solution.

\subsection{Functionalities}

The description of system behaviour or functionalities is possible by grouping goals, subgoals, percepts, actions and data together. Following this combination, each functionality 
is briefly described. Because the agent system is located within a dynamic environment, an understanding of how the system interacts with the environment is also required. Therefore, any data used by the agent system are defined at this initial stage. There are two types of data, one that is external to the agent system, and one that is internal. A message which contains information about the local environment, as obtained by a sensor, is considered as external data. If the message contained information generated by another agent within the programme then this data is considered internal. Particular attention is paid to external data, as this requires some form of interface.

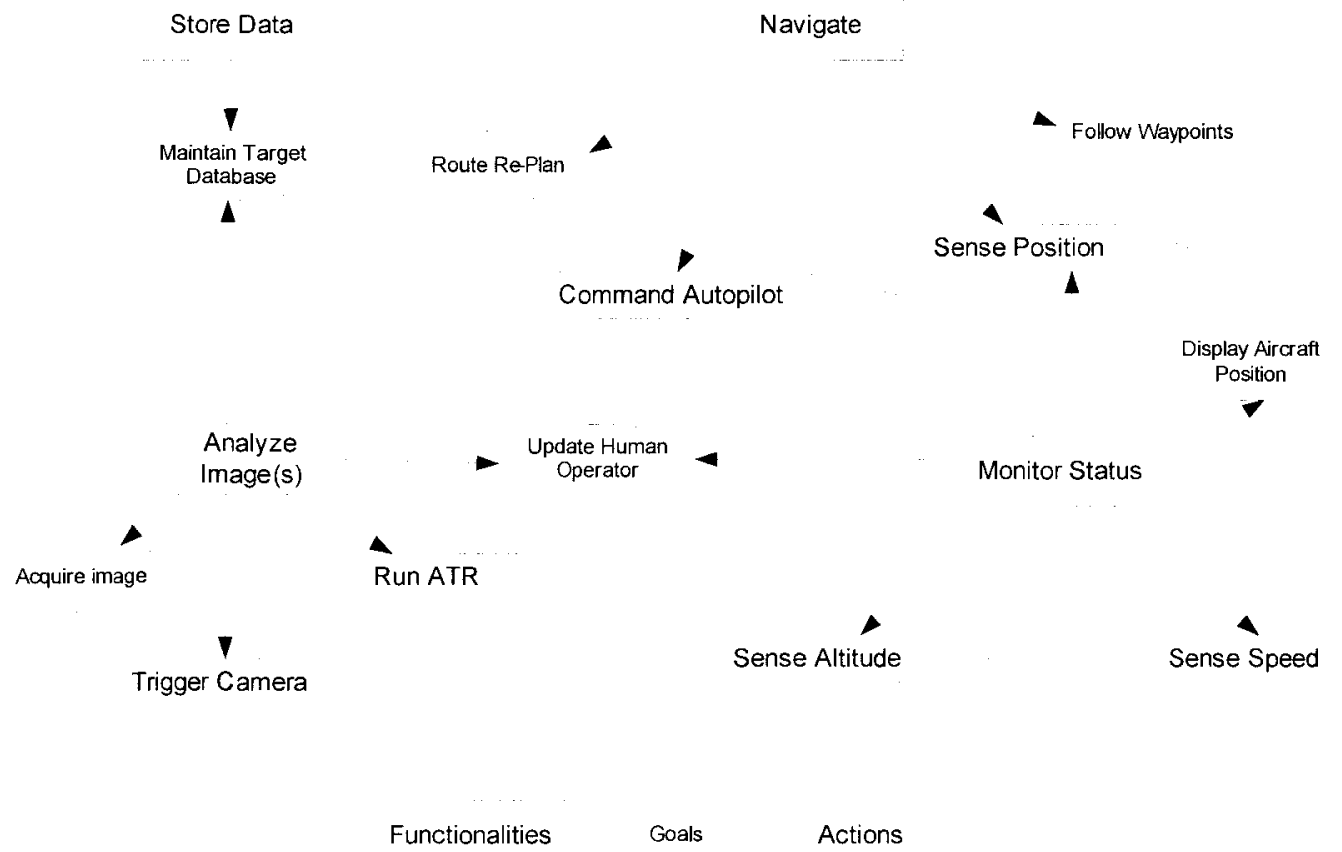

Figure 6-5: Coupled functionalities within the UAV System

Figure 6-5 shows there are four basic functionalities of the UAV system.

The monitor status functionality monitors aircraft states and transmits this information to the human operators. 
The navigate functionality maintains information about the current position of the vehicle and controls this position as required. It uses two goals for following existing waypoints and developing new points through route re-planning, while monitoring the aircraft position and controlling the autopilot system.

The store data functionality maintains a local database of target positions and the associated confidence values.

The goal of maintaining up to date information is augmented by the analyze images functionality, which uses the goal of acquiring images. This functionality triggers the camera, and updates the human operator with a combination of target and/or photograph information, once the ATR algorithm has been executed.

\subsection{Percepts}

As indicated in Figure 6-2, four percepts are directly related to the functionality of the autopilot system, where altitude, attitude, airspeed, and location measurements are required to ensure proper control over the aircraft at all times. Because these measurements are currently obtained by all UAV autopilot systems, it is assumed that any necessary signal conditioning to remove noise has been carried out. Furthermore, two of the percepts shown in Figure 6-2 apply to the acquisition and processing of target images.

Of these six percepts, only four are directly applicable to the intelligent software: air speed, vehicle position, altitude, and output from the ATR. 


\subsection{Actions}

Controlling the UAV remains a low-level function of the autopilot system; however, commanding altitudes and setting waypoints are the responsibilities of the agent-oriented system. Triggering the camera is another necessary action in order to acquire images. Throughout all phases of the mission, maintaining human operator awareness of all agent-decisions and vehicle actions is of paramount interest.

\subsection{Data Coupling}

There are multiple ways to group any set of functionalities into a single agent. Grouping is done when functionalities are related or require the same information. The only time that functionalities cannot be grouped together is when they exist on different hardware platforms, or when they are clearly not related.

Data coupling diagrams are used to visualize the interactions between functionalities and all of the necessary data. Arrows that are pointed towards a functionality indicate data that is used by the functionality, whereas arrows that point away, represent data produced by the functionality. Double-headed arrows represent a functionality that both produces and uses data. For example, the navigate function both creates and retrieves information from the waypoints database. 


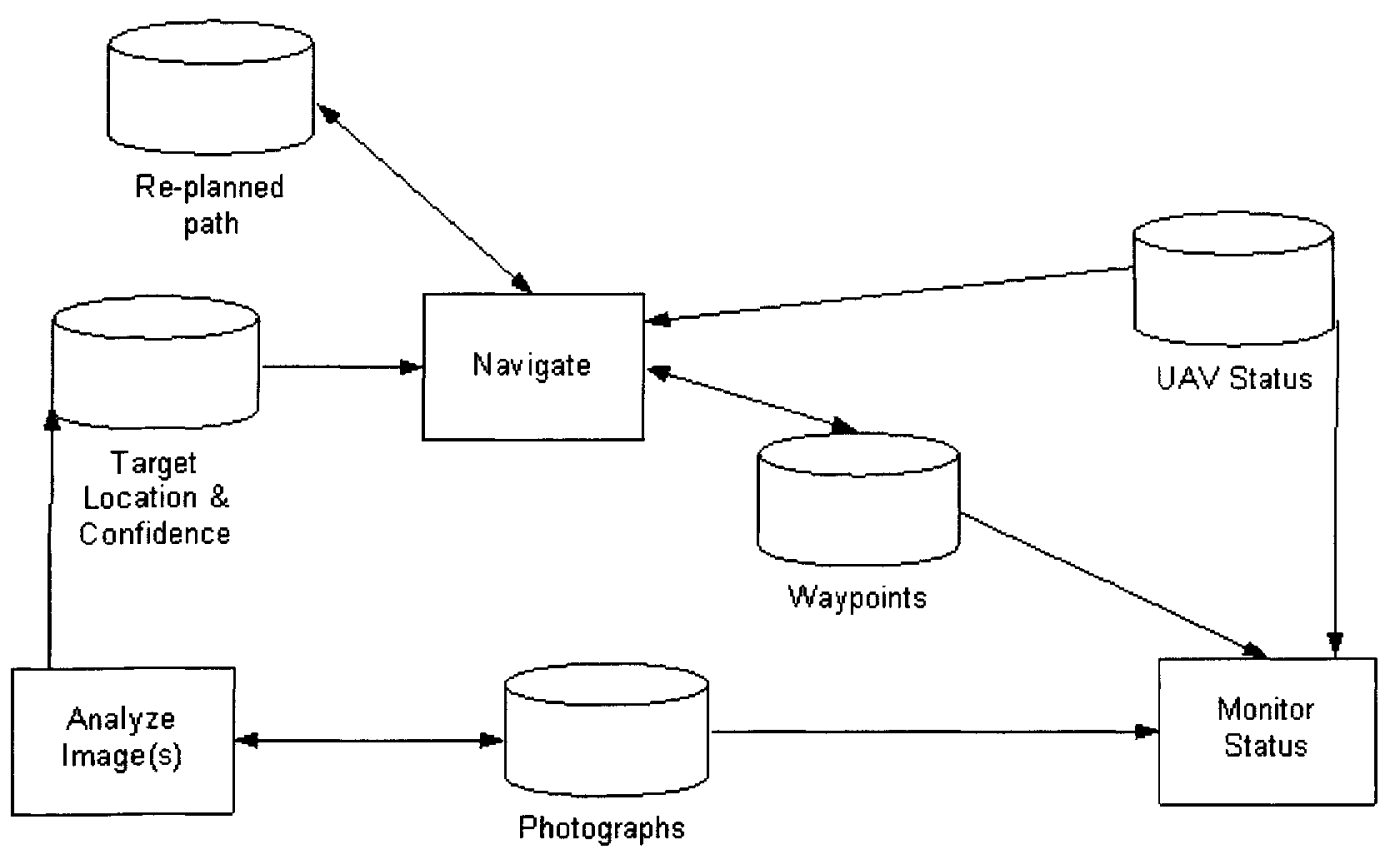

Figure 6-6: Functionality grouping with data sources

As defined by Padgham and Winikoff (2004), "coupling is a property of a group of components. The coupling of components is the extent to which they depend on one another. Low coupling yields a system in which changes are more likely to be localized. High coupling yields a system in which changing one component is likely to require changes to many other components." By these definitions, the navigate functionality shown in Figure 6-6, is highly coupled because the functionality impacts a number of other components. While this is not necessarily a problem, the functionality should be broken down into smaller components in order to better map agents to functions in later sections.

\subsection{Agent Descriptors}

It is desirable to link like-agents together where they can share and exchange information without jeopardizing the stability of the entire system. It is for this reason that the 
navigate functionality must be broken down into sub-functions. The sub-functions separate the decision-making, feedback monitoring, and command of the aircraft functions into three separate areas.

Using an agent acquaintance diagram it is possible to visualize the relationships among all agents. Within the system described by Figure 6-2, three interfaces with external systems are shown, including the ATR algorithm, ground control station (via the datalink), and the autopilot system. For each interface, an individual agent is proposed. Aside from managing external information, two additional agents are proposed to coordinate actions within the agent programme. As such, a five-agent solution was developed. While other approaches and agent configurations might also work, the arrangement described in Figure 6-6, was studied in this project.

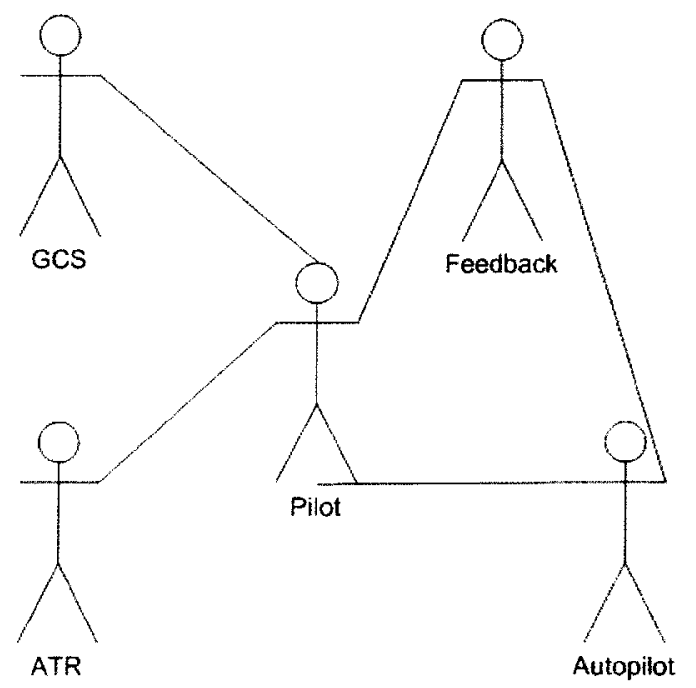

Figure 6-7: Agent acquaintance diagram

It is worthwhile to note that links within the acquaintance diagram are not directional. While the layout within Figure 6-7 is similar to the star pattern, it is also consistent with a distributed structure. When decisions are made, feedback is required to ensure that the 
intended action is being carried out, and appropriate results are obtained. Descriptions of the five agents are listed in Tables 5 - 9.

Table 5: GCS Agent Summary

\begin{tabular}{|l|l|}
\hline Agent Name & GCS \\
\hline Goals & $\begin{array}{l}\text { Maintain operator situational awareness, identify and update vehicle } \\
\text { position, altitude, vehicle speed and all decisions made by the agents }\end{array}$ \\
\hline Percepts & None \\
\hline Actions & Display information \\
\hline Data & UAV Status, Waypoint Information \\
\hline
\end{tabular}

Table 6: ATR Agent Summary

\begin{tabular}{|l|l|}
\hline Agent Name & ATR \\
\hline Goals & Handle results from ATR algorithm, record location of all spotted targets \\
\hline Percepts & ATR algorithm, image acquisition system \\
\hline Actions & Send messages to Pilot Agent \\
\hline Data & Record target information \\
\hline
\end{tabular}

Table 7: Pilot Agent Summary

\begin{tabular}{|l|l|}
\hline Agent Name & Pilot \\
\hline Goals & $\begin{array}{l}\text { Handle results from ATR algorithm, enable route re-planning, take } \\
\text { pictures of targets, and handle waypoint files that are consistent with } \\
\text { SAR manual search patterns. }\end{array}$ \\
\hline Percepts & Messages from ATR agent, messages from Feedback monitor \\
\hline Actions & Toggle ATR, send commands to Autopilot, relay updates to GCS \\
\hline Data & Route re-planning, waypoint information, target information \\
\hline
\end{tabular}

Table 8: Feedback Agent Summary

\begin{tabular}{|l|l|}
\hline Agent Name & Feedback \\
\hline Goals & Maintain intended flight pattern \\
\hline Percepts & $\begin{array}{l}\text { Vehicle position (GPS), vehicle speed, vehicle altitude, waypoint } \\
\text { information }\end{array}$ \\
\hline Actions & Messages to Autopilot and Pilot agents \\
\hline Data & Waypoint information, target information \\
\hline
\end{tabular}


Table 9: Autopilot Agent Summary

\begin{tabular}{|l|l|}
\hline Agent Name & Autopilot \\
\hline Goals & Control flight using aircraft's autopilot system \\
\hline Percepts & Messages from pilot and feedback agents \\
\hline Actions & Trigger camera, and send commands to autopilot system \\
\hline Data & Waypoint information, target information \\
\hline
\end{tabular}

\subsection{Interaction Diagrams}

The interaction diagram shows interactions among agents and is similar to diagrams used in object-oriented programming. Percepts and actions are also shown on the interaction diagrams to put the entire system in context. The interactions are entirely scenario dependent; therefore the following section is divided in three parts, with one diagram for each scenario (Figures 6-8 thru 6-10). Notation within each interaction diagram is standard, where time increases as one reads down the diagram, and each agent, percept, and action has a lifeline across the diagram. These diagrams help the designer prioritize the timing of the interactions between the various agents, and also provide a clear description of the inter-agent communications that take place during the mission.

Upon examination of Figure 6-8, the reader will notice that the mission begins when the GCS agent initially sends the search pattern to the Pilot agent. The Pilot agent stores this information and then instructs the autopilot agent to the next waypoint. As the vehicle travels from one point to the next, positional information is fed back to the Pilot agent via the Feedback agent. At set intervals (depending on vehicle speed), the Pilot agent instructs the Autopilot agent to trigger the camera. Once a snapshot has been obtained, the image is processed by the ATR algorithm and results are provided to the ATR agent. 
In the event that the processed image contains a target which has a high confidence value, the Pilot agent requests an update on the position of the vehicle from the Feedback Agent. Using the coordinates (lat/lon) of the target that were sent from the ATR agent to the Pilot Agent along with the vehicle's current position, the 'closer look' set of waypoints are generated by the Pilot agent and sent to the Autopilot agent. Once the new set of waypoints has been generated, the Pilot agent instructs the ATR agent to turn off the algorithm and updates the GCS agent of the current situation. At this point in time the 'closer look' plan is executed (see Section 6.2.3.5.1 for a detailed description). Following the completion of the plan, the original search pattern mission is continued. 


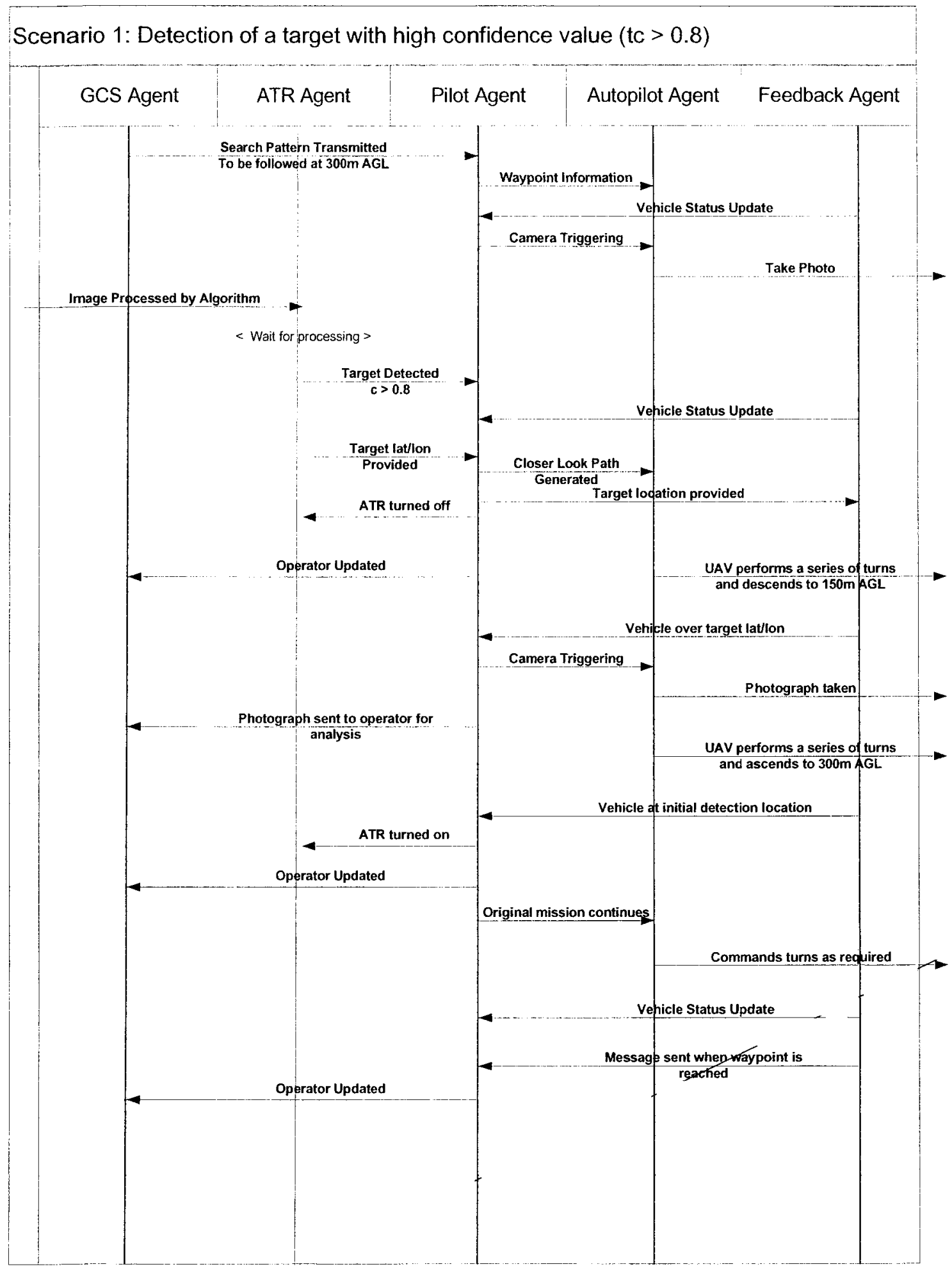

Figure 6-8: Scenario 1 interaction diagram 
Scenario 2: Detection of a target with medium confidence value $(0.4<\mathrm{tc}<0.8)$

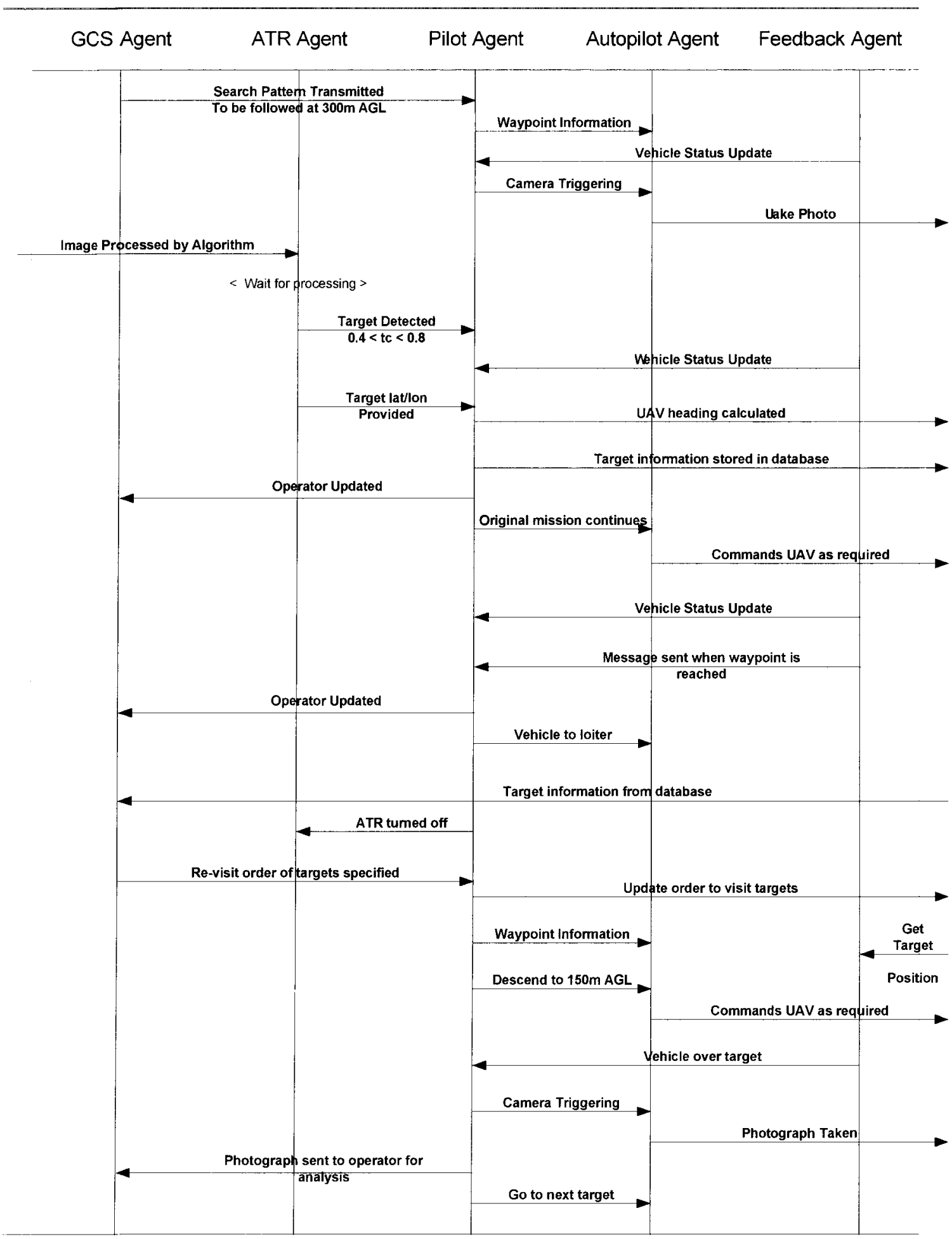

Figure 6-9: Scenario 2 interaction diagram 
Scenario 3: Detection of a target with low confidence value $(\mathrm{tc}<0.4)$

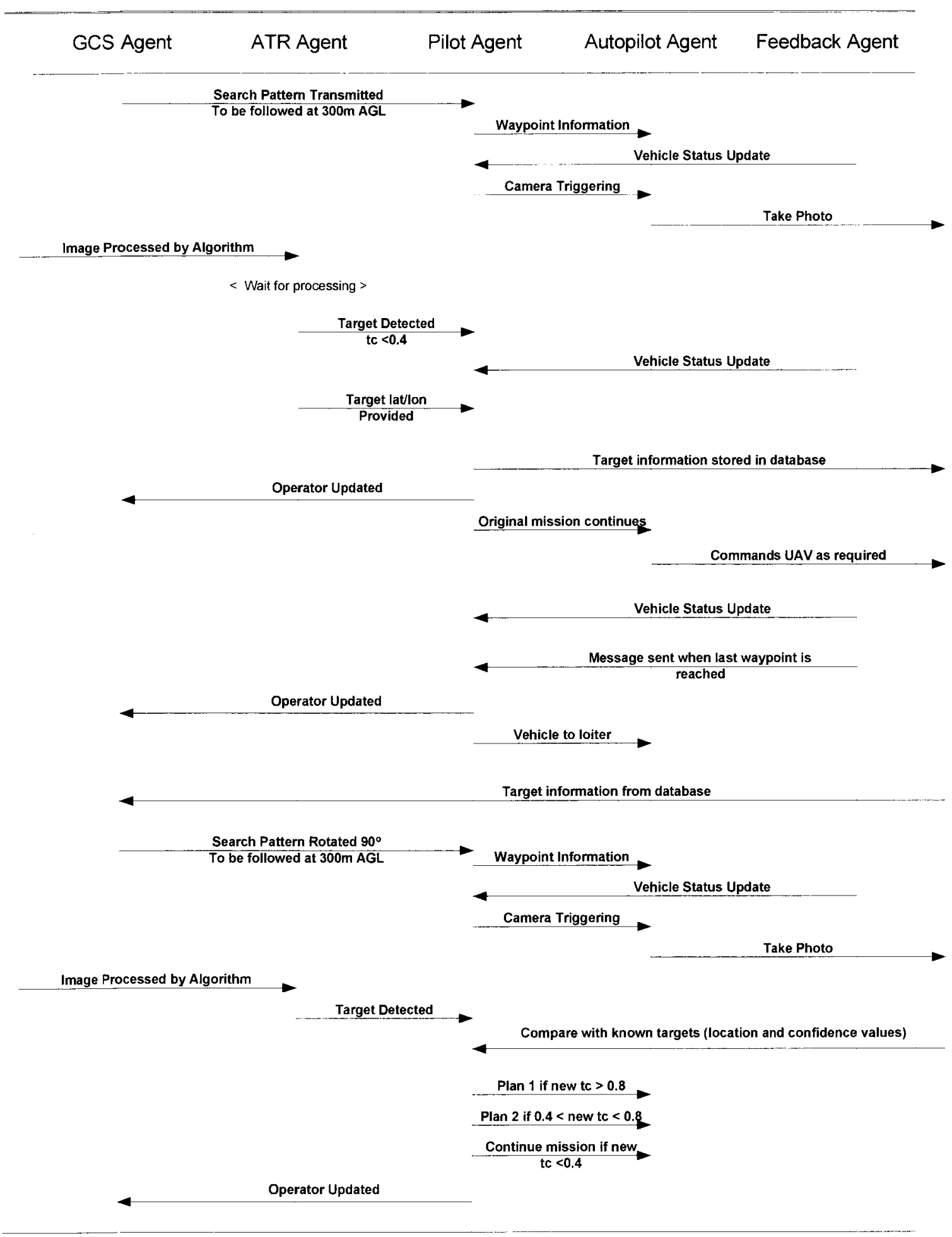

Figure 6-10: Scenario 3 interaction diagram 


\subsection{Agent System Overview}

Because the agent software is designed for use in a larger system of software and hardware within an actual air vehicle, system boundaries exist. The purpose of this section is to establish how the agent software interacts with other systems, and to provide details for the percepts and actions used by the individual agents.

\subsection{Boundaries of the Agent System}

The JACK programme interfaces with both hardware and software components which are identified by their IP addresses and a particular port number within the UAV network. Comparing the data link between the air vehicle and the ground station, to a long-range wireless network connection, it is reasonable to consider the GCS and proxy GCS agent as part of the UAV network. Any interactions beyond those that occur across the TCP/IP network, shown in Figure 6-11, are outside the boundaries of the agent system.

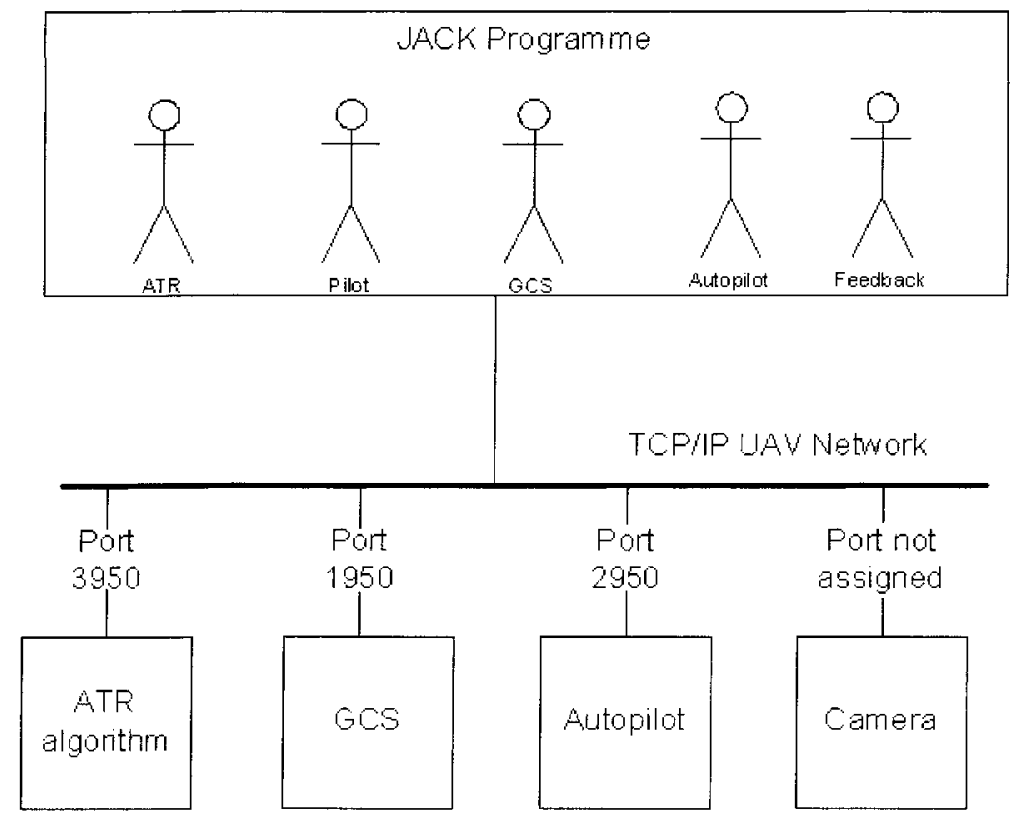

Figure 6-11: Software boundaries with external systems 


\subsection{Individual Percept Development}

This section includes details about the four percepts which are applicable to the JACK programme, and describes the relationship between percept and agent.

\section{Air Speed}

Vehicle air speed is monitored by the feedback agent, and commanded by the pilot agent. This measurement, when taken by the Micropilot MP2028 autopilot system, uses an air data probe to measure the velocity of the airflow. The measurement is then converted to a digital signal. The airspeed is provided in units of either feet/second or knots when using the default Imperial units or in kilometres per hour when using metric units. The measurements are updated five times per second throughout the duration of the mission. A GPS system could also be used to calculate airspeed through the differentiation of position.

\section{Vehicle Position}

The MP2028 autopilot system accepts both GPS and differential GPS data. As previously mentioned, GPS information can be used to establish vehicle airspeed. This is only possible by knowing the vehicle's position at any given point in time. There are two formats for measuring vehicle position. The first method uses positions relative to the point of system initialization. When the MP2028 is initialized prior to take-off it sets this point as $(0,0)$. Measurements relative to this position are measured as floating-points, in units of feet when set up in Imperial units or in metres when set up in metric units. It is also possible to make measurements in decimal degrees of latitude and longitude with 
precision to the fifth decimal place. During the course of a mission the feedback agent monitors vehicle position information, and the autopilot agent transmits this information when setting new waypoints.

\begin{abstract}
Altitude
While ultrasonic measurements are possible when flying close to the ground, the MP2028 may use GPS information to calculate vehicle altitude. Vehicle altitude is measured in units above sea level, and without using the differential GPS corrections, reading accuracy may vary in excess of 100 metres. As with vehicle speed and location, this information is used by the autopilot and feedback agents to control and monitor the progress of the air vehicle.
\end{abstract}

\begin{abstract}
ATR
The ATR agent receives information about target positions as calculated by third-party software for a given image. Depending on the probability of detection versus probabilityof-failure model that is chosen, targets are generated every eleven seconds for the simulation environment. Target information from the algorithm is contained within a 4byte string with the following format: $\langle\mathbf{T} *$ lat $|$ lon $|\mathbf{t c}\rangle$, where the pipe " $\mid$ "symbol is used to separate the values. Within the string, "T" identifies the string as containing target information, lat/lon identifies target position, and "tc" identifies the confidence value.
\end{abstract}




\subsection{Individual Action Development}

The agent software interacts with its environment through two primary actions. The first is by sending information to control the aircraft via the autopilot system, while the second action involves triggering the camera system to capture an image of the area beneath the aircraft.

\section{Control by the Autopilot System}

The ability to control the autopilot system in real-time is crucial to the success of using intelligent agents within an unmanned air vehicle. Failure to receive waypoint information, altitude information or airspeed information by the autopilot system will result in mission failure, and possibly, the loss of the vehicle. When waypoint information is sent to the autopilot system it is formatted similar to the target information, in a 4-byte string with the following format: $<\mathbf{W} *$ lat $\mid$ lon $\mid$ alt $>$ where "W" identifies the string as containing waypoint information. The latitude and longitude information can be sent as either distances, relative to the point of origin, or in decimal degrees of latitude and longitude. The final placeholder is for vehicle altitude at a particular waypoint. When a waypoint is sent to the autopilot system it is also sent to the feedback agent. While it is ultimately the responsibility of the autopilot hardware and built-in software to execute the command, the feedback agent monitors all situations.

\section{Triggering the Camera}

Real-time execution is required for triggering the camera to ensure that complete area coverage is achieved. Controlled by trigger pulses sent through a FireWire port from the 
onboard computer, images are acquired for further processing by the ATR algorithm. Failure to obtain the images will result in a mission's failure; however this failure does not have any impact on safety.

\subsection{Individual Data Development}

There are three independent databases currently used within the programme code, namely the database of known targets, locations of waypoints, and order of targets to revisit, which is referred to as the optimized path. The optimized path database records vehicle heading information, target location, and confidence values when targets of medium or greater -confidence values are found. This private database is updated throughout the mission, and interacts with numerous plans linked to the pilot, ATR, and autopilot agents. Initially the database does not contain any information. Once the initial search pattern has been flown and the last waypoint is reached, the locations within the database are shown to the GCS operator using the GCS agent. At this point in time, the operator chooses the order to revisit the locations. The rationale for this decision is provided in Section 6.2.4 under the current limitations of the code.

The target database contains similar information to the optimized path database except that the target database is public and is used and read directly by all the agents. The target database contains information (location and associated confidence values) that is used by all three algorithms described in Section 6.2.3.5. When the mission is first initialized, the target database is empty. 
The waypoint database is the final database used within the programme under development. It contains waypoint data provided initially from the search pattern file, and later includes waypoints necessary for the closer-look plan. Using the waypoint database, the feedback agent knows where the vehicle is headed, and the autopilot agent is able to retrieve the current waypoint.

\subsubsection{Bottom-Up Approach}

This requirements section introduces the bottom-up approach that was used in parallel to the top-down methods previously described. From the top-down analysis, a five-agent model was developed. As such, the requirements are sub-divided to reflect this configuration.

Without the time to perform a full system safety assessment as per RTCA Do-178B, it is reasonable to assume that because the intelligent agents are located between the core GCS software and the air vehicle autopilot system, a failure condition involving certain agents would likely result in the loss of the air vehicle. Some agents are not involved in the operation of the air vehicle and, while their failure could result in mission failure, this is not a safety issue. In the event of a safety-related failure, not all agent functions impact on the safety of the vehicle to the same degree, therefore only the most demanding levels of safety (A and B) are identified in the requirements listed below.

For each agent, individual requirements, rationale and an assignment of safety level has been provided in Tables 10-15. For the ease of notation, requirements are noted with the 


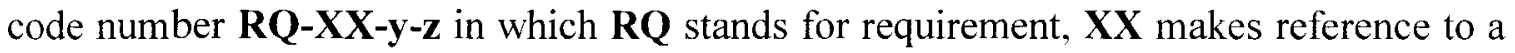
particular agent, $\mathbf{y}$ represents the requirement number, and $\mathbf{z}$ indicates a derived requirement.

$\mathrm{XX} \rightarrow \mathbf{P L}$ for Pilot related functional requirements

$\mathrm{XX} \rightarrow \mathbf{F M}$ for Feedback monitor related functional requirements

$\mathrm{XX} \rightarrow$ ATR for Automatic Target Recognition related functional requirements

$\mathrm{XX} \rightarrow \mathbf{A P}$ for Autopilot related functional requirements

$\mathrm{XX} \rightarrow \mathbf{G C S}$ for Ground control station related functional requirements

Table 10: Pilot Agent Requirements

\begin{tabular}{|c|c|c|c|}
\hline ID & Description & Rationale & Safety Level \\
\hline $\begin{array}{l}\text { RQ-PL- } \\
01\end{array}$ & $\begin{array}{l}\text { The pilot agent shall ensure that } \\
\text { the air vehicle flies at } 300 \mathrm{~m} \\
(1000 \mathrm{ft}) \text { above ground level } \\
\text { during the initial search over } \\
\text { the area of interest. }\end{array}$ & $\begin{array}{l}\text { Current SAR doctrine } \\
\text { requires that searches be } \\
\text { performed at three } \\
\text { altitudes: } 450 \mathrm{~m}(1500 \mathrm{ft}) \text {, } \\
300 \mathrm{~m}(1000 \mathrm{ft}) \text { and } 150 \mathrm{~m} \\
(500 \mathrm{ft})\end{array}$ & N/A \\
\hline $\begin{array}{l}\text { RQ-PL- } \\
02\end{array}$ & $\begin{array}{l}\text { The pilot agent shall ensure that } \\
\text { the air vehicle performs the } \\
\text { search at a constant velocity }\end{array}$ & $\begin{array}{l}\text { ATR processing times } \\
\text { require a minimum of } \\
11 \mathrm{sec} \text { per } 5 \mathrm{M} \text { pixel image. }\end{array}$ & $\mathrm{N} / \mathrm{A}$ \\
\hline $\begin{array}{l}\text { RQ-PL- } \\
03\end{array}$ & $\begin{array}{l}\text { The air vehicle shall be able to } \\
\text { fly any search pattern defined } \\
\text { in the National SAR Manual }\end{array}$ & $\begin{array}{l}\text { See Appendix A for search } \\
\text { pattern details }\end{array}$ & N/A \\
\hline $\begin{array}{l}\text { RQ-PL- } \\
03-1\end{array}$ & $\begin{array}{l}\text { The pilot agent must be able to } \\
\text { accept a series of waypoints as } \\
\text { defined by a human operator }\end{array}$ & $\begin{array}{l}\text { Search patterns are defined } \\
\text { by lat/lon points for the } \\
\text { start and end of search } \\
\text { lines }\end{array}$ & Level B \\
\hline $\begin{array}{l}\text { RQ-PL- } \\
03-2\end{array}$ & $\begin{array}{l}\text { The waypoint file shall be } \\
\text { readable in relative metres or in } \\
\text { latitude / longitude }\end{array}$ & Interface requirement & $\mathrm{N} / \mathrm{A}$ \\
\hline $\begin{array}{l}\text { RQ-PL- } \\
04\end{array}$ & $\begin{array}{l}\text { The pilot agent shall be able to } \\
\text { communicate with all other } \\
\text { agents }\end{array}$ & Inter-agent communication & Level A \\
\hline $\begin{array}{l}\text { RQ-PL- } \\
05\end{array}$ & $\begin{array}{l}\text { The pilot agent shall command } \\
\text { the air vehicle to fly at } 150 \mathrm{~m} \\
\text { ( } 500 \mathrm{ft} \text { ) above ground level to } \\
\text { investigate all high probability }\end{array}$ & $\begin{array}{l}\text { High-resolution images are } \\
\text { required for all high } \\
\text { probability targets }\end{array}$ & $\mathrm{N} / \mathrm{A}$ \\
\hline
\end{tabular}




\begin{tabular}{|c|c|c|c|}
\hline & targets identified by the ATR & & \\
\hline $\begin{array}{l}\text { RQ-PL- } \\
05-1\end{array}$ & $\begin{array}{l}\text { The pilot agent shall trigger the } \\
\text { camera once over a possible } \\
\text { target }\end{array}$ & Interface requirement & N/A \\
\hline $\begin{array}{l}\text { RQ-PL- } \\
05-2\end{array}$ & $\begin{array}{l}\text { The pilot agent shall transmit } \\
\text { images of the target to the GCS } \\
\text { operators }\end{array}$ & $\begin{array}{l}\text { Closer Look algorithm (see } \\
\text { Section } 6.2 .3 .5 .1 \text { ) }\end{array}$ & N/A \\
\hline $\begin{array}{l}\text { RQ-PL- } \\
06\end{array}$ & $\begin{array}{l}\text { A message shall be displayed } \\
\text { when each waypoint is reached }\end{array}$ & Situational awareness & Level B \\
\hline $\begin{array}{l}\text { RQ-PL- } \\
06-1\end{array}$ & $\begin{array}{l}\text { Once an entire search areas has } \\
\text { been covered, the pilot agent } \\
\text { shall inform the GCS operators }\end{array}$ & Situational awareness & N/A \\
\hline $\begin{array}{l}\text { RQ-PL- } \\
07\end{array}$ & $\begin{array}{l}\text { After reaching the last } \\
\text { waypoint, the pilot agent shall } \\
\text { instruct the autopilot agent to } \\
\text { maintain a } 5^{\circ} \text { bank angle and } \\
\text { loiter about the last waypoint. } \\
\text { The ATR agent shall be } \\
\text { instructed to turn off the } \\
\text { algorithm while loitering. }\end{array}$ & & N/A \\
\hline $\begin{array}{l}\text { RQ-PL- } \\
08\end{array}$ & $\begin{array}{l}\text { The pilot agent shall have a } \\
\text { number of different plans of } \\
\text { action depending on the } \\
\text { confidence value generated by } \\
\text { the ATR }\end{array}$ & & N/A \\
\hline $\begin{array}{l}\text { RQ-PL- } \\
08-1\end{array}$ & $\begin{array}{l}\text { The closer look plan shall be } \\
\text { used when target confidence } \\
\text { values (tc) }>0.8 \text { on a scale } \\
\text { from } 0 \text { to } 1\end{array}$ & $\begin{array}{l}\text { Closer Look Plan } \\
\text { (see Section 6.2.3.5.1) }\end{array}$ & $\mathrm{N} / \mathrm{A}$ \\
\hline $\begin{array}{l}\text { RP-PL- } \\
08-2\end{array}$ & $\begin{array}{l}\text { The optimized path plan shall } \\
\text { be used for targets with } \\
\text { confidence values } 0.4<(\mathrm{tc})<0.8\end{array}$ & $\begin{array}{l}\text { Optimized Path Plan } \\
\text { (see Section 6.2.3.5.2) }\end{array}$ & $\mathrm{N} / \mathrm{A}$ \\
\hline $\begin{array}{l}\text { RP-PL- } \\
08-3\end{array}$ & $\begin{array}{l}\text { A general overview pattern } \\
\text { plan shall be used for targets } \\
\text { with confidence values (tc) }<0.4\end{array}$ & $\begin{array}{l}\text { General Overview Plan } \\
\text { (see Section 6.2.3.5.3) }\end{array}$ & $N / A$ \\
\hline $\begin{array}{l}\text { RP-PL- } \\
09\end{array}$ & $\begin{array}{l}\text { If the ATR confidence factor is } \\
\text { (tc) }>0.8 \text { the closer look plan } \\
\text { shall be used immediately, } \\
\text { during the execution of any } \\
\text { plan }\end{array}$ & $\begin{array}{l}\text { Immediate investigation of } \\
\text { probable targets is } \\
\text { consistent with current } \\
\text { SAR practices }\end{array}$ & $\mathrm{N} / \mathrm{A}$ \\
\hline $\begin{array}{l}\text { RP-PL- } \\
10\end{array}$ & $\begin{array}{l}\text { As the air vehicle loiters having } \\
\text { completed the mission, the pilot } \\
\text { agent shall send a message to } \\
\text { the GCS indicating the location } \\
\text { of targets } 0.4<(\mathrm{tc})<0.8 \text {, and } \\
\text { request that the GCS operator }\end{array}$ & & N/A \\
\hline
\end{tabular}




\begin{tabular}{|l|l|l|l|}
\hline $\begin{array}{l}\text { RP-PL- } \\
10-1\end{array}$ & $\begin{array}{l}\text { The pilot agent shall command } \\
\text { survey these targets } \\
\text { the air vehicle to descend to } \\
150 \mathrm{~m} \text { AGL before flying to the } \\
\text { target locations }\end{array}$ & $\begin{array}{l}\text { Flying at half-the original } \\
\text { altitude will increase pixels } \\
\text { on target, and prevent } \\
\text { unnecessary fuel burn for } \\
\text { ascents and descents }\end{array}$ & Level B \\
\hline $\begin{array}{l}\text { RP-PL- } \\
10-2\end{array}$ & $\begin{array}{l}\text { Upon completion of this search, } \\
\text { the air vehicle shall return to } \\
\text { the start location and loiter until } \\
\text { receiving further instruction }\end{array}$ & & Level B \\
\hline
\end{tabular}

Table 11: Feedback Monitoring Agent Requirements

\begin{tabular}{|c|c|c|c|}
\hline ID & Description & Rationale & Safety Level \\
\hline $\begin{array}{l}\text { RQ-FM- } \\
01\end{array}$ & $\begin{array}{l}\text { The feedback monitoring agent } \\
\text { shall keep in contact with the } \\
\text { autopilot and pilot agent }\end{array}$ & Inter-agent communication & Level A \\
\hline $\begin{array}{l}\text { RQ-FM- } \\
01-1\end{array}$ & $\begin{array}{l}\text { The feedback agent shall } \\
\text { communicate vehicle position } \\
\text { with the pilot agent }\end{array}$ & Inter-agent communication & Level B \\
\hline $\begin{array}{l}\text { RQ-FM- } \\
02\end{array}$ & $\begin{array}{l}\text { The feedback agent shall } \\
\text { ensure that a constant vehicle } \\
\text { speed is maintained }\end{array}$ & $\begin{array}{l}\text { Camera timing correlated } \\
\text { with vehicle speed to } \\
\text { ensure complete coverage }\end{array}$ & Level B \\
\hline $\begin{array}{l}\text { RQ-FM- } \\
03\end{array}$ & $\begin{array}{l}\text { The feedback agent shall } \\
\text { ensure that a constant altitude } \\
\text { is maintained }\end{array}$ & & Level A \\
\hline $\begin{array}{l}\text { RQ-FM- } \\
04\end{array}$ & $\begin{array}{l}\text { The feedback agent shall } \\
\text { ensure that intended waypoints } \\
\text { are reached }\end{array}$ & & Level A \\
\hline
\end{tabular}


Table 12: Autopilot Agent Requirements

\begin{tabular}{|l|l|l|l|}
\hline ID & Description & Rationale & Safety Level \\
\hline RQ-AP-01 & $\begin{array}{l}\text { The autopilot agent shall } \\
\text { communicate with the pilot } \\
\text { and feedback agents }\end{array}$ & Inter-agent communication & Level A \\
\hline RQ-AP-02 & $\begin{array}{l}\text { The autopilot agent shall } \\
\text { communicate with the } \\
\text { autopilot hardware to control } \\
\text { the air vehicle }\end{array}$ & Interface Requirement & Level A \\
\hline $\begin{array}{l}\text { RQ-AP-02- } \\
1\end{array}$ & $\begin{array}{l}\text { The autopilot agent shall be } \\
\text { able to adjust vehicle velocity }\end{array}$ & $\begin{array}{l}\text { Desired velocity is a } \\
\text { typical input for UAV } \\
\text { autopilots }\end{array}$ & Level B \\
\hline $\begin{array}{l}\text { RQ-AP-02- } \\
2\end{array}$ & $\begin{array}{l}\text { The autopilot agent shall be } \\
\text { able to adjust vehicle altitude } \\
\text { by transmitting desired value } \\
\text { to the hardware }\end{array}$ & $\begin{array}{l}\text { Desired altitude is a typical } \\
\text { input for UAV autopilots }\end{array}$ & Level A \\
\hline $\begin{array}{l}\text { RQ-AP-02- } \\
\text { The autopilot agent shall be } \\
\text { able to adjust vehicle position } \\
\text { by transmitting waypoint } \\
\text { information (lat/lon) to the } \\
\text { hardware }\end{array}$ & $\begin{array}{l}\text { Waypoint navigation is } \\
\text { typical for UAV autopilots }\end{array}$ & Level A \\
\hline
\end{tabular}

Table 13: Automatic Target Recognition Agent Requirements

\begin{tabular}{|c|c|c|c|}
\hline ID & Description & Rationale & Safety Level \\
\hline $\begin{array}{l}\text { RQ-ATR- } \\
01\end{array}$ & $\begin{array}{l}\text { The ATR agent shall be able } \\
\text { to communicate with the } \\
\text { Pilot agent }\end{array}$ & Inter-agent communication & N/A \\
\hline $\begin{array}{l}\text { RQ-ATR- } \\
01-1\end{array}$ & $\begin{array}{l}\text { A message shall be displayed } \\
\text { when a target has been } \\
\text { detected }\end{array}$ & Situational awareness & N/A \\
\hline $\begin{array}{l}\text { RQ-ATR- } \\
02\end{array}$ & $\begin{array}{l}\text { The ATR agent shall be able } \\
\text { to communicate with the } \\
\text { ATR algorithm }\end{array}$ & Interface Requirement & N/A \\
\hline $\begin{array}{l}\text { RQ-ATR- } \\
03\end{array}$ & $\begin{array}{l}\text { The position of all possible } \\
\text { targets shall be recorded } \\
\text { onboard the air vehicle }\end{array}$ & & $\mathrm{N} / \mathrm{A}$ \\
\hline $\begin{array}{l}\text { RQ-ATR- } \\
03-1\end{array}$ & $\begin{array}{l}\text { For each target the } \\
\text { confidence value shall also } \\
\text { be recorded }\end{array}$ & & $\mathrm{N} / \mathrm{A}$ \\
\hline $\begin{array}{l}\text { RQ-ATR- } \\
04\end{array}$ & $\begin{array}{l}\text { The ATR algorithm will } \\
\text { produce false positives. The } \\
\text { agent must act on all possible } \\
\text { targets detected by the }\end{array}$ & $\begin{array}{l}\text { False alarm rate likely to } \\
\text { be } 1 \text { false alarm per image } \\
\left.(2 \% \text { per degree })^{2}\right)\end{array}$ & $\mathrm{N} / \mathrm{A}$ \\
\hline
\end{tabular}




\begin{tabular}{|l|l|l|l|}
\hline & algorithm & & \\
\hline RQ-ATR- & $\begin{array}{l}\text { The ATR agent shall record } \\
\text { the position of all targets } \\
\text { identified by the algorithm }\end{array}$ & $\begin{array}{l}\text { Positional accuracy from } \\
\text { ATR } \pm 20 \mathrm{~m} \text { to } \pm 50 \mathrm{~m}\end{array}$ & \\
\hline $\begin{array}{l}\text { RQ-ATR- } \\
06\end{array}$ & $\begin{array}{l}\text { The ATR agent shall } \\
\text { communicate only when } \\
\text { details from the algorithm } \\
\text { are available }\end{array}$ & $\begin{array}{l}\text { Algorithm requires 11 } \\
\text { seconds to process a 5 } \\
\text { Mega-Pixel image }\end{array}$ & N/A \\
\hline
\end{tabular}

Table 14: Ground Control Station Agent Requirements

\begin{tabular}{|l|l|l|l|}
\hline ID & Description & Rationale & Safety Level \\
\hline $\begin{array}{l}\text { RQ-GCS- } \\
01\end{array}$ & $\begin{array}{l}\text { GCS agent shall communicate } \\
\text { with the Pilot agent }\end{array}$ & $\begin{array}{l}\text { Inter-agent } \\
\text { communication }\end{array}$ & Level A \\
\hline $\begin{array}{l}\text { RQ-GCS- } \\
02\end{array}$ & $\begin{array}{l}\text { GCS agent shall communicate } \\
\text { with the core GCS software }\end{array}$ & Interface Requirement \\
\hline $\begin{array}{l}\text { RQ-GCS- } \\
03\end{array}$ & $\begin{array}{l}\text { GCS agent shall be able to } \\
\text { graphically communicate data } \\
\text { from the air pilot agent to the } \\
\text { human operator }\end{array}$ & Level A \\
\hline $\begin{array}{l}\text { RQ-GCS- } \\
04\end{array}$ & $\begin{array}{l}\text { GCS agent shall be able to } \\
\text { receive air vehicle data and } \\
\text { images, simultaneously }\end{array}$ & $\begin{array}{l}\text { Network bandwidth will } \\
\text { permit a 3MB image to be } \\
\text { transmitted in 30s }\end{array}$ & Level A \\
\hline
\end{tabular}

Having developed the top-level scenarios, goals, and high-level functionalities, along with the bottom-up requirements and safety considerations, the initial system specification phase is complete. The next phase of the Prometheus design model involves using the outcome from this phase for the detailed design of the multi-agent system.

\subsubsection{Detailed Design}

Within the JACK Development Environment (JDE), there are two methods for developing a programme. The first method requires an understanding of how individual agents, plans, databases and capabilities relate to each other. Using the tools provided in 
the JDE, it is possible to graphically lay out the structure of the programme. During this process, JACK automatically generates a skeleton code which ensures that naming conventions are maintained. Editing a variable name on the main screen automatically updates every instance within the rest of the programme. This method was used in the development of the programme because it provided the most straightforward transition from architecture layout to actual coding using the bottom-up approach.

Alternatively, the second method for developing a programme uses plan graphs. This method is typically used for following reasoning models, and does not take into account the relationships among elements of the programme. This method is used to work through the reasoning processes. An example of a process specification plan graph is described in Section 6.2.3.2 where the decision reasoning process is described for a target spotted after being photographed and analyzed by the ATR algorithm.

\subsubsection{Agent Overview Diagrams}

During the first phase of development, multiple individual plans were used in the programme rather than clustering these together into capabilities. Initially, the agents used plans and databases to achieve the mission objectives. However as the programme became more complex with overlap among various plans, the role for capabilities emerged. The following section illustrates the bottom-up design process that was used to connect agents to databases, plans and capabilities. 


\subsection{Pilot Agent}

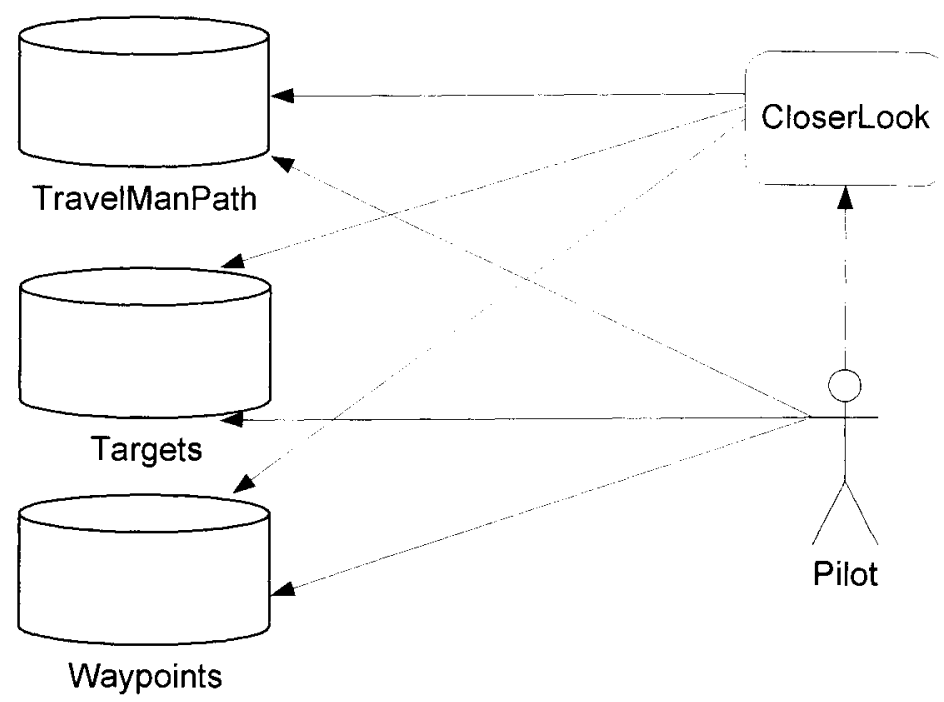

Figure 6-12: Pilot agent overview

Rather than having a direct coupling among agents, as shown in Figure 6-7, the pilot agent interacts with other agents through databases, as shown in Figure 6-12. Information, which is gathered by the other agents, is used to update the databases throughout the course of the mission. 


\subsection{Feedback Agent}

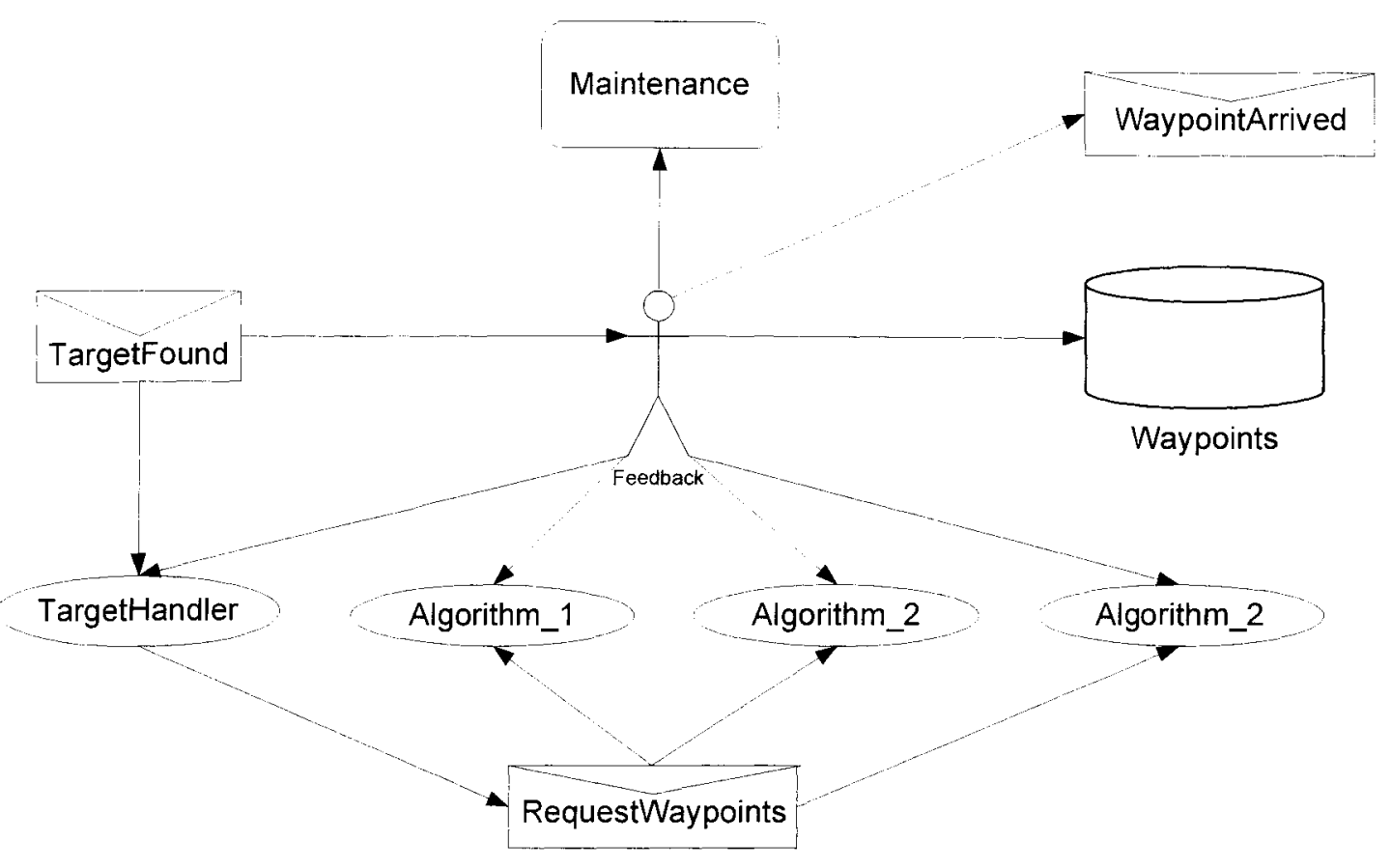

Figure 6-13: Feedback monitoring agent overview

The feedback agent shown in Figure 6-13 uses waypoint information and algorithms to determine where the aircraft should be headed based on the active plan and waypoint information. Also, it compares the calculated position with the actual vehicle position. Once the desired waypoint has been reached, it sends a message to the pilot agent. Upon receipt of the message, the pilot agent uses the waypoint database and sends a new waypoint to the autopilot agent. 


\subsection{A utopilot Agent}

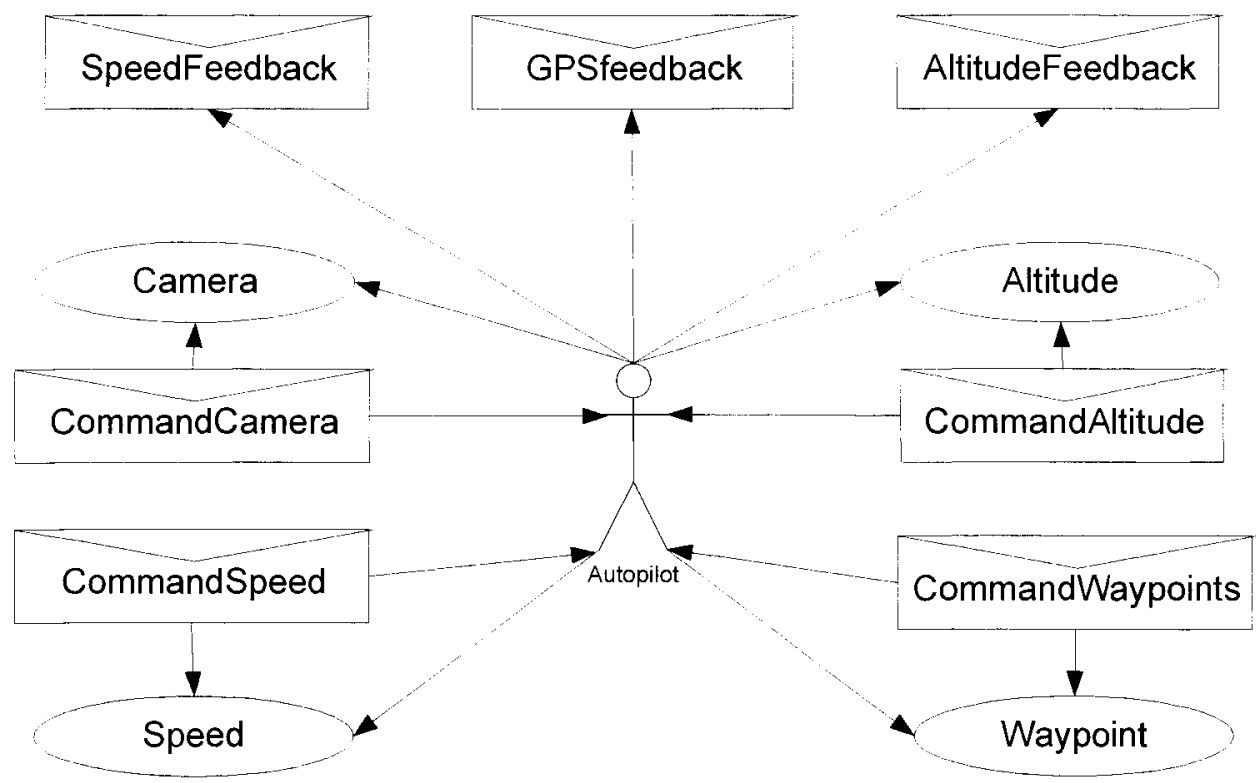

Figure 6-14: Autopilot agent overview

The autopilot agent in Figure 6-14 sends waypoint commands and altitude commands to the autopilot system, and also handles feedback from these systems. Feedback information is transmitted as messages from the autopilot agent to the feedback agent.

\subsection{ATR Agent}

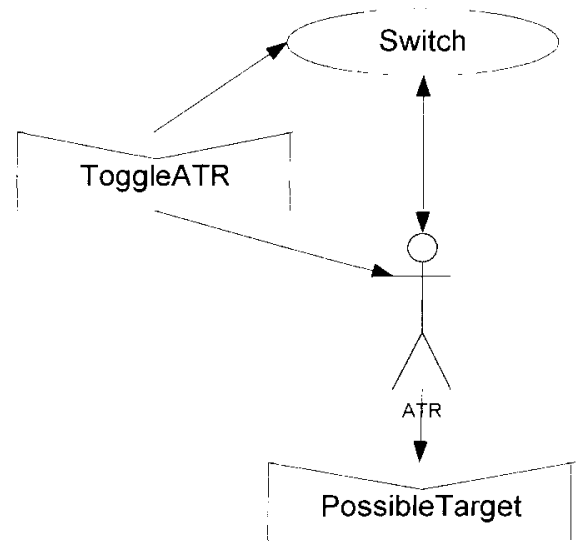

Figure 6-15: ATR agent overview 
The ATR agent shown in Figure 6-15 can be toggled on or off using the plan called "switch". Otherwise, the agent simply relays information from the ATR algorithm in a format that the other agents within the programme can read.

\subsection{GCS Agent}

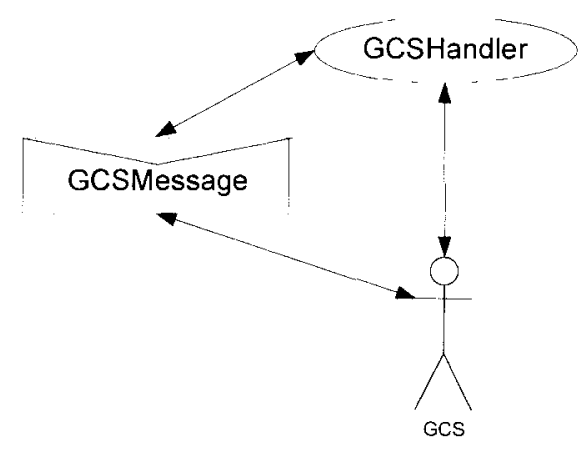

Figure 6-16: GCS agent overview

As with the ATR agent, the GCS agent's interactions with the larger system are quite simple. The GCS agent shown in Figure 6-16 communicates with the agents onboard the air vehicle through a series of messages.

This design method requires an incremental approach, followed by multiple revisions and iterations. One key advantage of developing the program using this tool is that the graphical description of the software reinforces how the various components interact.

As the various databases, agents, capabilities and plans are connected using this method, skeleton code is also generated. By drawing arrows between components, the necessary calls are added to the software routines. These automated features of JACK ease troubleshooting the code when it is compiled. 


\subsubsection{Process Specification}

The alternative to graphical layout is a method of developing software architectures through plan graphs. These diagrams are similar to the one shown in Figure 6-17 for deciding what to do if a target is found. The diagram works through a logical reasoning process. This method is ideally suited to instances where the objective is to model human behaviour or follow prescribed methods and doctrines. When the programme designed with these process maps is executed, it is possible to follow through the agent's reasoning process. This is particularly beneficial when debugging the programme, or when the agent's decision is contrary to what a human operator would have chosen.

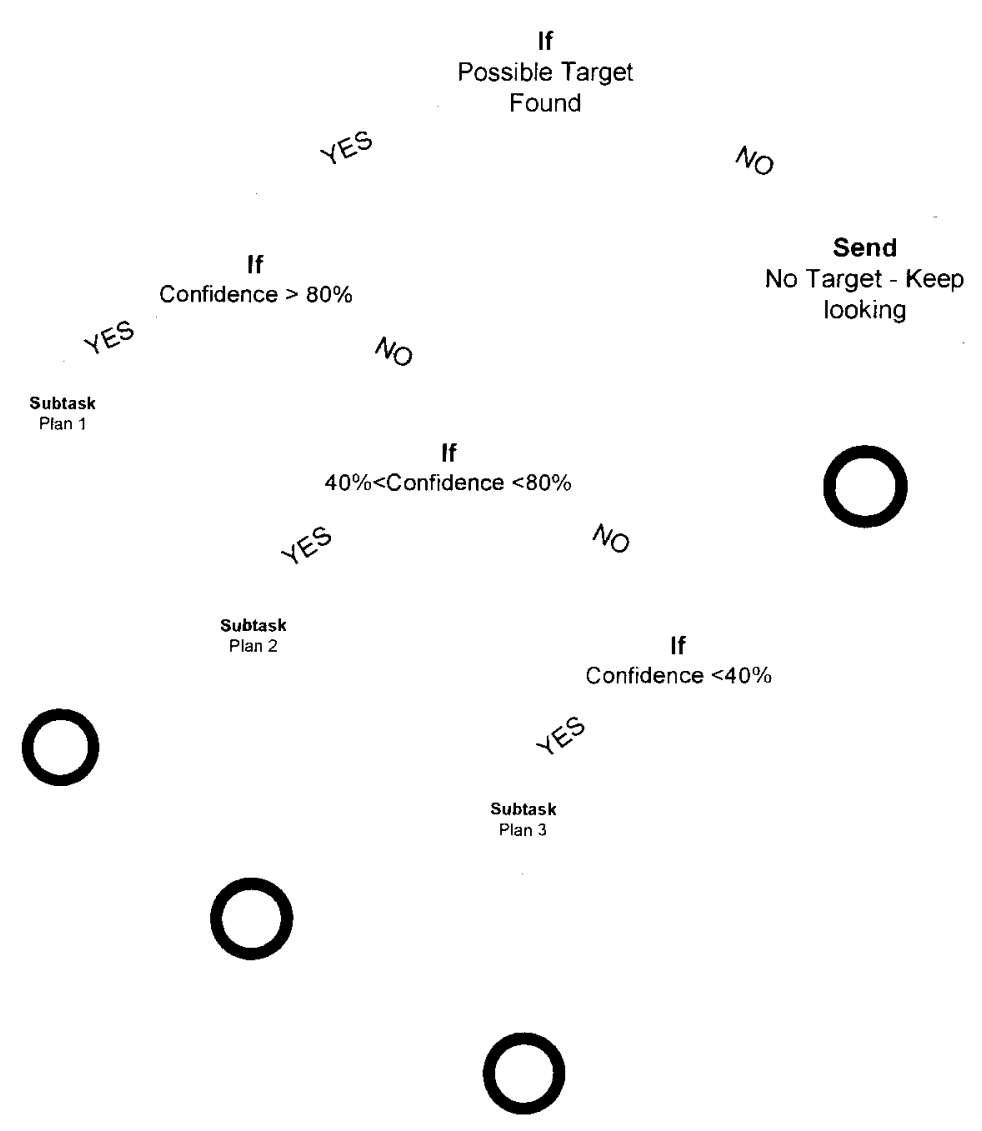

Figure 6-17: Plan mapping with start and end states 


\subsubsection{Capability Overview Diagram}

As previously mentioned, capabilities are ideally suited for repetitive tasks. Each capability is able to send and receive messages, use data and execute plans. In the described programme, two capabilities were created. One capability is responsible for the maintenance of flight states, and is associated with the feedback agent. The second capability is associated with the closer-look scenario for identifying high-probability targets.

\subsection{Maintenance Capability}

The maintenance capability provides the means to maintain the desired track, speed and altitude for the vehicle. The capability does not use any internal goals. Integrated into the early stages of the programme, this capability establishes the necessary foundation for future developments of the software. Health monitoring is but one example where this maintenance capability could be used in future developments. Currently, the capability handles three incoming messages, SpeedFeedback, AltitudeFeedback and GPSFeedback, and is able to command changes through the CommandSpeed and CommandAltitude messages. These messages are used in combination with three plans, MaintainSpeed, MaintainAltitude and GPShandler. These plans monitor the speed, altitude and position of the vehicle, and command changes if the desired values are not consistent with the present vehicle state. Two outgoing messages are generated by the maintenance capability, and include: the ability to set the vehicle velocity and altitude through CommandSpeed and CommandAltitude. 


\subsection{Closer-Look Capability}

The closer-look capability involves the ability to send and receive numerous messages and interact with all three algorithms. It is enacted whenever a high probability target is found by the ATR. Not only does the capability include external messages to other agents, but it also includes two internal messages.

The capability is first initialized by the PossibleTarget message that identifies the position of a high probability target. During the execution of the closer look algorithm, described as Algorithm_1 in Section 6.2.3.5.1, numerous waypoints are generated. Other incoming messages include: GCSMessage which handles control messages between the GCS and the Pilot agent, WaypointArrived which is sent from the Feedback agent to the Pilot agent when a waypoint has been reached, RequestWaypoint which identifies the next waypoint, Loitering which is sent to the Autopilot system once the last waypoint is reached, and the ResumeAndEnd message which is sent when the resume waypoint has been reached indicating that the closer look search is complete, or alternatively indicated that the mission is complete. Both the ResumeAndEnd and Loitering messages are internal messages, meaning that they trigger activities within the capability rather than among other agents in the programme.

Outgoing messages from this capability include ToggleATR that turns off the ATR once the closer look search is in progress and then turns the algorithm back on once the search is complete. The CommandCamera and TargetFound messages are both related to acquiring the target, just as CommandWaypoints is used to generate the closer look 
search pattern. When the closer look capability is used, the GCS is updated using the GCSMessage. The closer look capability is also able to issue RequestWaypoint messages. Furthermore, the capability uses all three databases, Targets, Waypoints, and TravelManPath for the travelling salesman or optimized path series of waypoints.

Finally, the capability is able to enact a series of plans including FlyCloserLookWaypoints, ResumeMission, Algorithm_1 (see Section 6.2.3.5.1), GCSconnection, FlySearchWaypoints, Algorithm_2 (see Section 6.2.3.5.2), RequestPath, InitialPath, Algorithm_3 (see Section 6.2.3.5.3), and InitialOverview. Details of each plan are provided in the indicated section along with their mathematical development.

\subsubsection{Plan Descriptors}

Twenty plans are used within the programme; each plan is triggered by data from a percept or from a database. Often the plans involve receiving information and then acting on this information. It is through plans that actions are possible. Table 15 summarizes the twenty plans.

\section{Table 15: Plan Descriptions}

\begin{tabular}{|l|l|}
\hline Plan ID & Plan-01 Algorithm_1 \\
\hline Description & Used to calculate the closer look flying pattern \\
\hline Trigger & RequestWaypoints (message / event) \\
\hline Plan ID & Plan-02 FlyCloserLookWaypoints \\
\hline Description & Plan to fly closer look pattern \\
\hline Trigger & PossibleTarget (message / event) \\
\hline Actions & $\begin{array}{l}\text { Sends event: ToggleATR } \\
\text { Sends event: TargetFound }\end{array}$ \\
\hline Uses Data & Targets \\
\hline
\end{tabular}




\begin{tabular}{|c|c|}
\hline Produces Data & Targets \\
\hline Plan ID & Plan-03-FlySearchWaypoints \\
\hline Description & $\begin{array}{l}\text { This is the capability to command the air vehicle to follow specific } \\
\text { waypoints }\end{array}$ \\
\hline Trigger & WaypointArrived \\
\hline Actions & $\begin{array}{l}\text { Posts event: ResumeAndEnd } \\
\text { Sends event: CommandCamera } \\
\text { Sends event: RequestWaypoints } \\
\text { Posts event: RequestWaypoints } \\
\text { Sends event: GCSMessage }\end{array}$ \\
\hline Uses Data & Waypoints \\
\hline Plan ID & Plan-04-GCSconnection \\
\hline Description & This plan handles messages from the GCS \\
\hline Trigger & GCSMessage \\
\hline Actions & $\begin{array}{l}\text { Posts event: ResumeAndEnd } \\
\text { Sends event: CommandCamera } \\
\text { Sends event: RequestWaypoints } \\
\text { Posts event: RequestWaypoints } \\
\text { Sends event: GCSMessage }\end{array}$ \\
\hline Uses Data & TravelManPath \\
\hline Produces Data & TravelManPath (updates database) \\
\hline Plan ID & Plan-05-Maintain Altitude \\
\hline Description & Command autopilot to adjust altitude \\
\hline Trigger & AltitudeFeedback \\
\hline Actions & Sends event: CommandAltitude \\
\hline Plan ID & Plan-06-MaintainSpeed \\
\hline Description & Command autopilot to adjust speed \\
\hline Trigger & SpeedFeedback \\
\hline Actions & Sends event: CommandSpeed \\
\hline Plan ID & Plan-07-Switch \\
\hline Description & $\begin{array}{l}\text { Turns the ATR on and off corresponding to the message received } \\
\text { from the pilot }\end{array}$ \\
\hline Trigger & ToggleATR \\
\hline Actions & Toggle the workings of the ATR (enable/disable) \\
\hline Plan ID & Plan-08-ResumeMission \\
\hline Description & $\begin{array}{l}\text { This plan is used to resume the mission after investigating a target } \\
\text { or to end the mission }\end{array}$ \\
\hline Trigger & ResumeAndEnd \\
\hline Actions & Sends event: ToggleATR \\
\hline Uses Data & Targets \\
\hline Plan ID & Plan-09-Camera \\
\hline Description & $\begin{array}{l}\text { Handles commands to the camera for taking high-resolution } \\
\text { pictures }\end{array}$ \\
\hline Trigger & CommandCamera \\
\hline Uses Data & Uses the interface to the autopilot or Firewire system \\
\hline
\end{tabular}




\begin{tabular}{|l|l|}
\hline Plan ID & Plan-10-WayPoint \\
\hline Description & Sends the next waypoint to the autopilot \\
\hline Trigger & CommandWaypoints \\
\hline Actions & Uses the interface to the autopilot \\
\hline Plan ID & Plan-11-Speed \\
\hline Description & $\begin{array}{l}\text { This plan sends a speed adjustment command to the autopilot to } \\
\text { either increase or decrease the speed depending on the target versus } \\
\text { actual speed of the aircraft }\end{array}$ \\
\hline Trigger & CommandSpeed \\
\hline Actions & $\begin{array}{l}\text { Uses the interface through the autopilot for the throttle control } \\
\text { which will be used to change the speed of the aircraft }\end{array}$ \\
\hline Plan ID & Plan-12-Altitude \\
\hline Description & $\begin{array}{l}\text { This plan sends altitude adjustments commands to the autopilot as } \\
\text { part of the maintenance system }\end{array}$ \\
\hline Trigger & CommandAltitude \\
\hline Actions & $\begin{array}{l}\text { Uses the interface through the autopilot for elevator deflections, } \\
\text { where the elevator is used for altitude changes }\end{array}$ \\
\hline Plan ID & Plan-13-GPShandler \\
\hline Description & This is used to handle GPS feedback \\
\hline Trigger & GPSFeedback \\
\hline Actions & Uses interface within the FeedBackMonitor agent \\
\hline Plan ID & Plan-14-TargetHandler \\
\hline Description & Handles the Target information \\
\hline Trigger & TargetFound \\
\hline Actions & $\begin{array}{l}\text { Sends event: RequestWaypoints } \\
\text { Posts event: RequestWaypoints } \\
\text { Uses the interface within the FeedBackMonitor agent }\end{array}$ \\
\hline Plan-15-Algorithm_2 \\
\hline Plan ID & $\begin{array}{l}\text { This calculates the new order of waypoints while the UAV is } \\
\text { circling around the end point. }\end{array}$ \\
\hline Description & RequestWaypoints \\
\hline Trigger & Uses the agent implementing the InternalAgent class \\
\hline Uses Data & Plan-16-RequestPath \\
\hline Plan ID & $\begin{array}{l}\text { lssues commands to the autopilot from the pilot at the loitering } \\
\text { point and requests the optimized search path from the GCS }\end{array}$ \\
\hline Description & $\begin{array}{l}\text { Targets } \\
\text { Sonds event: RequestWaypoints } \\
\text { Posts event: RequestWaypoints }\end{array}$ \\
\hline Trigger & $\begin{array}{l}\text { Sosts event: Loitering } \\
\text { Actions }\end{array}$ \\
\hline Uses Data & argets \\
\hline
\end{tabular}




\begin{tabular}{|c|c|}
\hline Plan ID & Plan-17-GCSHandler \\
\hline Description & $\begin{array}{l}\text { Handles GCSmessages to transfer the order of the search path data } \\
\text { between the GCS and the pilot }\end{array}$ \\
\hline Trigger & GCSMessage \\
\hline Actions & Sends event: GCSMessage \\
\hline Uses Data & Uses the interface to the GCS \\
\hline Plan ID & Plan-18-InitialPath \\
\hline Description & $\begin{array}{l}\text { This plan processes and requests input to the optimized or traveling } \\
\text { salesman path before the path is executed. }\end{array}$ \\
\hline Trigger & Loitering \\
\hline Actions & $\begin{array}{l}\text { Posts event: Loitering } \\
\text { Sends event: RequestWaypoints } \\
\text { Posts event: RequestWaypoints } \\
\text { Sends event: ToggleATR }\end{array}$ \\
\hline Uses Data & TravelManPath \\
\hline Plan ID & Plan-19-Algorithm_3 \\
\hline Description & $\begin{array}{l}\text { This plan lets the agents search using the existing search pattern } \\
\text { once it has been rotated } 90 \text { degrees }\end{array}$ \\
\hline Trigger & RequestWaypoints \\
\hline Uses Data & Uses an agent implementing InternalAgent \\
\hline Plan ID & Plan-20-InitialOverview \\
\hline Description & $\begin{array}{l}\text { This plan initializes the agent to begin the overview search for the } \\
\text { waypoint pattern that has been rotated } 90 \text { degrees }\end{array}$ \\
\hline Trigger & Loitering \\
\hline Actions & $\begin{array}{l}\text { Sends event: ToggleATR } \\
\text { Sends event: CommandWaypoints }\end{array}$ \\
\hline
\end{tabular}

\subsubsection{Underlying Algorithms}

As identified by the various plans described in Table 15, vehicle behaviour is ultimately dependent on the outcome from the ATR algorithm (target location and confidence values) and the search pattern that is being followed. Flight conditions are considered to be ideal, with no wind effects or other navigational difficulties. Once a waypoint file for the search pattern has been uploaded to the vehicle, following the points is a relatively straightforward process. This section describes the details of all three algorithms which correspond to different target confidence values, as shown in Table 16. 
Table 16: Algorithms for Various Target Confidence Values

\begin{tabular}{|c|c|}
\hline $\begin{array}{c}\text { Target } \\
\text { Confidence }\end{array}$ & $\begin{array}{c}\text { Algorithm } \\
\text { Used }\end{array}$ \\
\hline$>0.8$ & 1 \\
\hline $0.4 \leq 0.8$ & 2 \\
\hline$<0.4$ & 3 \\
\hline
\end{tabular}

\subsection{Closer Look (Algorithm_I)}

Upon identification and location of a possible target with a corresponding confidence factor that is greater than 0.8 , the $A T R$ agent sends a message to the pilot agent. This message triggers the pilot agent to choose one of the closer look plans that allows the vehicle to obtain a high-resolution image of the target.

It is assumed that the identification process and messaging are completed in near realtime. As with any computation there is some degree of lag within the system. Until this lag is fully characterized, a five second buffer has been added at the beginning of the Closer Look algorithm, as described by the six steps below and illustrated by Figures 618 and 6-19. The steps assume the autopilot system is able to hold the vehicle on a straight path between waypoints irrespective of wind effects.

As the trigger message is sent to the pilot agent, the vehicle will continue to travel along the original flight path. During this time the ATR agent is disabled. The Closer-Look plan includes $100 \mathrm{~m}$ of forward travel before a right turn is required. Assuming the vehicle is travelling at $20 \mathrm{~m} / \mathrm{s}$, this provides the initial five second buffer. After traveling $100 \mathrm{~m}$, a $180^{\circ}$ turn is performed by the vehicle, while maintaining a constant altitude. The 
turn assumes a maximum bank angle of $20^{\circ}$ (per Section 5.1.2.3) for a forward velocity of $20 \mathrm{~m} / \mathrm{s}$. Following the turn, the vehicle descends from $300 \mathrm{~m}$ (1000 feet) to $150 \mathrm{~m}$ (500 feet). During this maneuver, the vehicle descends with a maximum nose-down attitude of $20^{\circ}$ (per Section 5.1.2.3).

A second $180^{\circ}$ turn is completed and the vehicle then flies straight and level at 150 metres, en route to the target. The approach path aligns the vehicle, such that the target is centered directly along the centreline of the fuselage. With a vertically mounted highresolution camera, the target is also centred within the field of view of the camera.

As the vehicle passes over the coordinates of the target, the camera takes a number of photos in rapid succession, to compensate for possible errors in target geo-location and rotation.

After over-flying the target, the reverse procedure is followed while the vehicle returns to an altitude of 305 metres.

The vehicle is then realigned to fly along the original search path, before reaching the initiation point of the Closer-Look algorithm.

At this point, the ATR agent is reactivated, and the vehicle continues to follow the original search pattern. 


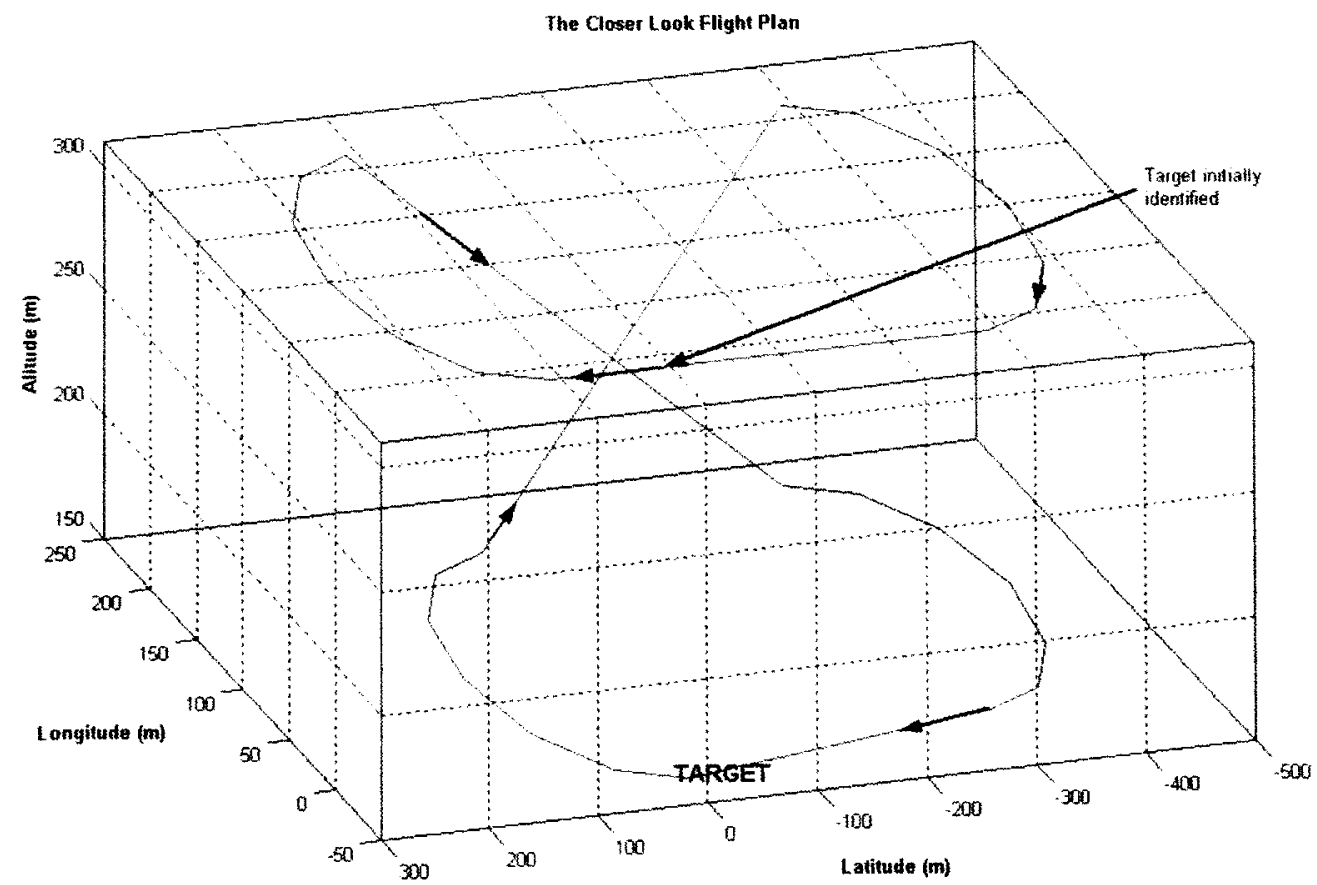

Figure 6-18: UAV flight profile following detection of a possible target.

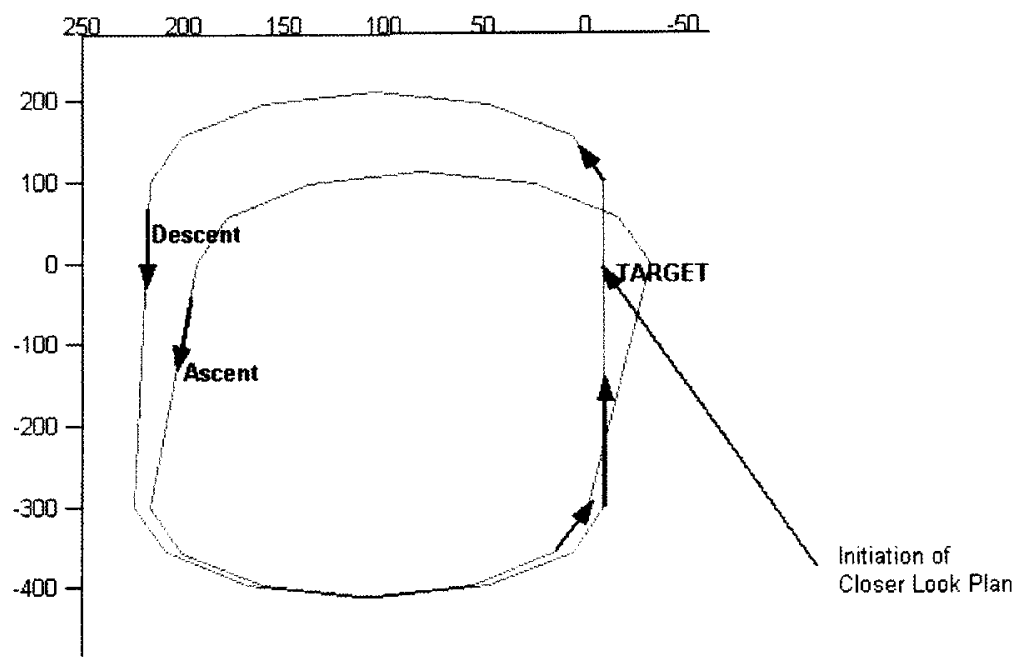

Figure 6-19: The closer-look flight plan, as seen from above. 


\subsection{Algorithm_1 mathematics}

A turn radius of $112 \mathrm{~m}$ assumes a maximum bank angle of $20^{\circ}$, for a vehicle moving at $20 \mathrm{~m} / \mathrm{s}$. The calculation is as follows, where:

$\frac{V}{R}=\frac{g}{V} \tan \phi$

For a velocity of $\mathrm{V}=20 \mathrm{~m} / \mathrm{s}$, and a maximum bank angle of $\phi=20^{\circ}$ (as prescribed by the autopilot system), the minimum radius of turn $\mathrm{R}_{\min }$ can be found after solving the rate of the turn.

$$
\begin{aligned}
& \omega=\frac{9.81 \mathrm{~m} / \mathrm{s}^{2}}{20 \mathrm{~m} / \mathrm{s}} \tan 20=0.1785 / \mathrm{s} \\
& R_{\min }=\frac{V}{\omega}=\frac{20 \mathrm{~m} / \mathrm{s}}{0.1785 / \mathrm{s}}=112 \mathrm{~m}
\end{aligned}
$$

\subsection{Optimized Path (Algorithm_2)}

When a possible target is located, with a confidence factor between 0.4 and 0.8 , it is important that the heading information be included with the position information that is generated by the ATR algorithm. The following algorithm calculates this heading information by using the positions of the previous and next waypoints. Corrections for wind direction are not required because the camera is pointing straight down and so the camera should be following the straight-line track between the two waypoints. However, image rotation (vehicle yaw) has not been accounted for. Furthermore, if the camera were fixed on the nose of the air vehicle, then accounting for wind direction would be required. 
Determining Heading

Given by Figure 6-20:

$\mathrm{L}_{1}=$ starting latitude

$\mathrm{L}_{2}=$ ending latitude

$\lambda_{1}=$ starting longitude

$\lambda_{2}=$ ending longitude

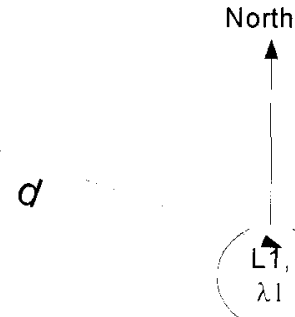

Figure 6-20: Heading between points

\section{Distance}

The distance between two points (d) can be calculated assuming the great circle effect of flying on a sphere, which is more accurate than Pythagorean theory. These calculations are based on equations provided in AFR 51-40: Air Navigation (Department of Defense, 1989).

d is calculated in nautical miles $(\mathrm{nm})$, where $1 \mathrm{~nm}=1.85200 \mathrm{~km}$

$$
\begin{aligned}
& d=60 \cos ^{-1}\left[\sin L_{1} \cdot \sin L_{2}+\cos L_{1} \cdot \cos L_{2} \cos \left(\lambda_{2}-\lambda_{1}\right)\right] \\
& D=d(\mathrm{~nm}) \times 1.852\left(\frac{\mathrm{km}}{\mathrm{nm}}\right)
\end{aligned}
$$

\section{Heading}

$$
\begin{aligned}
& H_{i j}=\cos ^{-1}\left[\frac{\sin L_{2}-\sin L_{1} \cdot \cos \left(\frac{d}{60}\right)}{\sin \left(\frac{d}{60}\right) \cdot \cos L_{1}}\right] \\
& \Theta_{i j}= \begin{cases}H_{i j}, & \sin \left(\lambda_{2}-\lambda_{1}\right)<0 \\
360^{\circ}-H_{i j}, & \sin \left(\lambda_{2}-\lambda_{1}\right) \geq 0\end{cases}
\end{aligned}
$$

Where the heading $\Theta_{\mathrm{ij}}$ is measured in degrees, clockwise from true north. 


\subsection{General Overview (Algorithm_3)}

Once all the low probability targets have been flagged, and the entire search pattern has been flown $300 \mathrm{~m} \mathrm{AGL}$, rotate the search pattern $90^{\circ}$ and perform the search again while still at $300 \mathrm{~m}$. As before if a target comes up with a confidence value $>0.8$ check it out immediately. If the confidence value is $<0.8$ compare the target location to those previously recorded in the database. If the confidence value has increased over the value in the database, use Algorithm_2 to obtain an image of the location. If the target has already been photographed, ignore the location and continue with the mission.

\subsubsection{Future Capabilities}

The optimized path currently relies on operator input to establish the optimum route to revisit the points. Initially it was thought that the problem could be treated as a "travelling salesman" problem; however, the optimal solution includes many constraints, making the problem more complex than initially thought. The problem is complicated because the route that minimizes the distance between target locations, as calculated by solving a travelling salesman problem, does not take into consideration approach direction, minimum turn radius, or sun angles. In the event that the target is spotted with a confidence value of 0.78 , the best direction to approach the target is likely to be the same direction, or $180^{\circ}$ to the path being travelled when it was first spotted. Second, if two targets are located close to each other, it may not be possible to maneuver the vehicle quickly enough to align the camera system over the second target. Finally, depending on the sun angle, it may be undesirable to approach a target from one direction over another. 
At the present time, the targets are only visited in the order that the operator decides, and the system does not take into consideration any of the aforementioned limitations. In order to solve this problem, a rule-based optimization algorithm is required, along with appropriate sensors for determining sun angles to avoid shadows.

\subsubsection{Future Implications to $\mathrm{C}^{3}$ Issues}

Any information that is required to initialize the agent's beliefset, such as vehicle performance values, vehicle systems, sub-systems and sensors, must be initially input by the operator into databases that are stored within both the GCS and the air vehicle instances of JACK. Thereby, all agents within the system will start with the same basic understanding of the world around them. Because the intelligent agents are making decisions independently of the human operators, it is imperative that the human operators remain alert and maintain a sufficient level of situational awareness of the UAV's activities. If a decision has been made by the agents onboard the air vehicle, then information regarding the triggers and the final decision should be presented to the ground operator on a secondary screen. Depending on the nature of the decision, a box requesting input from the operator may be required. In the event that there is a lack of convergence or agreement about the decision, the operator must be able to reject the agent's decision and take control of the air vehicle. 


\subsection{Implications to Standards}

For intelligent agents to be integrated within a Canadian Forces ground control station, as would be found in a search and rescue environment, an understanding of operational requirements is necessary. This section details the implications of an autonomous mission management programme to STANAG 4586.

In order for the JACK intelligent agents to work within the air vehicle, a second instance of the JACK software must also exist within the ground control station. Inter-agent communication can then occur between agents that are located at the GCS and those that are passed from the agents on board the air vehicle. The agents within the GCS are responsible for receiving information from the air vehicle and interacting with the hardware and software components within the GCS to ensure that the information is passed to the human operators.

Information exchange between the UAV and GCS takes place through the data link interface. This interface provides the communication pathway for messages between the ground instance and the air instance of the intelligent agents. This concept is important, because the intelligent agents are effectively removing the need for a vehicle specific module (VSM), which is found with the architecture of STANAG 4586. Figure 6-21 shows this modification as the addition of two JACK intelligent agent bars on either side of the data link interface from the original figure shown in Chapter 2. 

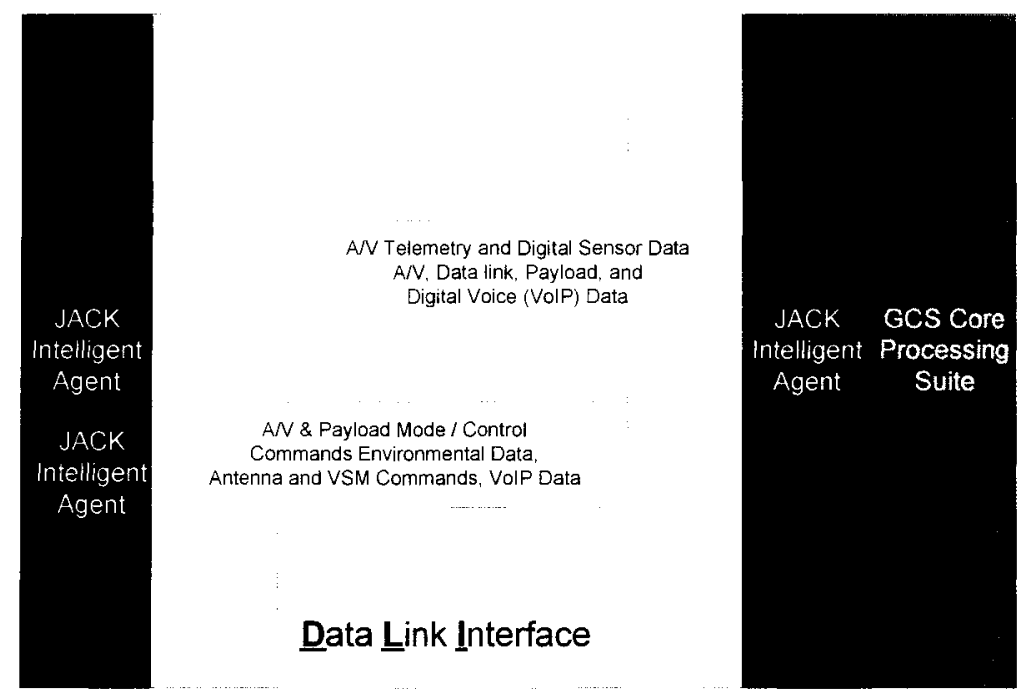

Figure 6-21: High-Level depiction of DLI content (adapted from STANAG 4586)

Under the current setup of STANAG 4586 the VSM is responsible for

- Managing the real-time control interactions with the air vehicle,

- Translating data that were sent between the air vehicle and the GCS, and

- Acting as a database or server for vehicle specific data, such as performance or configuration data.

By using JACK intelligent agents, vehicle control is managed in real time by the agentsoftware. The sensors onboard the air vehicle and the human / computer interface on the GCS are inputs to the intelligent agent software. Therefore, it is only through the JACK program that information is exchanged between the vehicle's systems and the operators. Using the databases within the JACK software it is possible to store specific platform data. For example, an agent's beliefset may contain air vehicle performance data if the agent is required to control the air vehicle. Similarly, any sensor-based monitoring 
activities must be defined in the JACK code. In the event that the data link is lost, the agent-oriented software must determine the most appropriate course of action. Therefore, to someone who is familiar with the use of VSMs, JACK intelligent agents not only replaces much of this function, but also augments the required capabilities! In order to fully replace the VSM through the use of intelligent agents, a substantially more involved agent programme is required; one that monitors and reports on the health of vehicle systems, and permits an operator to select different modes of operations. Provided that all sensor data was available in digital rather than analogue format, imagery databases could be setup through the agent program. Section 1.4 of STANAG 4586 already considers the possibility for VSMs to become an airborne component. In this event the Ground Data Terminal, which is analogous to the GCS Agent, would have the following functionality:

- A hardware interface necessary to support the network connection the GCS over which DLI messages are exchanged.

- This is similar to the GCS agent communicating with the agents onboard the air vehicle.

- Sufficient processing capability to format outgoing messages and to parse incoming messages to/from the DLI formats.

- Inter-agent messages are already easily created and understood by other agents.

- The capability to distinguish data link commands and status messages from those that are intended for the airborne VSM, and the capability of interpreting data link 
commands and status without the assistance of the GCS. This could be achieved using more than one agent.

Future implementation of JACK within a STANAG 4586 environment is not only a logical next step, but also one that may be easily accommodated with a small investment of time and a few modifications to the standard.

For consistency, all information passed from the air vehicle to the GCS within the JACK environment should follow the message handling approach outlined in STANAG 4586. Under this guise, all positional references are expressed in the latitude-longitude system of the World Geodetic System (WGS-84) of degrees, using double precision floatingpoint numbers. The global geocentric reference frame (WGS-84) provides a single, common, accessible 3-dimensional coordinate system for global geospatial data. Through a series of improvements in 1994 and 1996, significant distortions and biases have been removed. For mapping, charting and en-route navigation applications these changes are negligible (less than $30 \mathrm{~cm}$ ) and therefore the WGS-84 standard should be considered unchanged (NIMA, 1997). STANAG 4586 also defines the format of messages or data streams. When information is passed from JACK to the simulator environment or to the GCS, it is important that the contents be consistent with this format as well. 


\subsection{Summary}

The programme developed in this chapter avoids the common pitfalls of agent-oriented programming, through careful planning, from requirements to system specification. Considerations for aircraft performance, hardware and software systems have been made in this design along with any limitations imposed by the SAR mission objectives.

The programme described in this chapter compiles and executes as intended when executed within the JACK environment; however, a method to visualize the programme controlling an air vehicle is highly desirable. This issue is addressed in Chapter 7, which describes the simulation environments that have been developed to accommodate testing of this software.

During the development of any software, and particularly when using intelligent agents, it is imperative that full documentation be created to record the decisions that were made during the development process. This is particularly important for agents since they will have the ability to make their own decisions. Traceability for the agent's decisions is crucial. The code developed for this thesis is fully commented, and further described in a separate document by Dai and Carryer (2005). 


\section{Chapter 7: Simulation}

\section{Environment}

\section{CHAPTER SUMMARY}

This chapter describes three simulation environments that were investigated to provide a visualization environment for the JACK program. Following an overview of each method's setup, advantages and limitations, a detailed description is provided of the necessary modifications to the core agent program. Simulation results are also provided where appropriate. Building on the lessons learned from each method, a way-forward is suggested. 


\subsection{Simulation Environment Development}

Testing within a synthetic environment is required to ensure that the decisions made by the intelligent agents are reasonable under the circumstances, and consistent with those that a human operator would make. Simulation not only allows validation that the software is functioning properly, but it also helps to identify areas for future improvements. Rather than just using the text output from the JACK software, the simulation environment provides additional visual cues that the program is functioning properly. Using models of the UAV platforms, along with geographical terrain data it is possible to better appreciate the implications of the decisions that are being made.

Many of the requirements presented in Chapter 6 resulted directly from interfacing needs between the agent programme and existing hardware and software found within an air vehicle system. Therefore, prior to integrating JACK into an air vehicle system, each interface must be tested in a simulation environment. This approach is consistent with RTCA Do- $178 \mathrm{~b}$ and with industry practices, as it is much safer and less costly than testing a program in-flight. For reasons of safety, minimizing development time, optimizing cost benefits, and increasing software functionality, it is essential to have a working simulation environment to test a UAV system with JACK mission control. This chapter describes three simulation environments that were explored to meet this need.

Currently, there are no specific UAV platforms or systems available to Carleton University to test an autonomous search and rescue mission; however, computer models of generic autopilots and aircraft are available. Furthermore, by using X-Plane, a flight 
simulation program, it is possible to model the external environment. X-Plane is a comprehensive flight simulator software programme produced by Laminar Research. One key feature of X-Plane is that it provides a UDP interface for sending commands to the programme and receiving data back from the programme. Using standard Ethernet communication, it is possible to either send commands to X-Plane's flight model or to completely override the flight model and directly set the aircraft states. For this reason, X-Plane was selected for this research project.

The first environment described in Section 7.1, is entirely JAVA based and involves interfacing a series of JAVA windows to represent actual software and hardware components that exist within a UAV system. The completed JAVA-based simulation environment demonstrates the full functionality of the decision-making ability of the JACK software; however, it does not include a model of an aircraft flying. The second environment described in Section 7.2 is based on a series of $\mathrm{C}++$ translator programs that interface JACK with X-Plane, while the third method described in Section 7.3 explores how an intermediary operating environment called HLA could be used.

Building an effective simulation environment for the JACK program requires a number of individually developed software programs to work together. As each of the three environments were studied, changes, additions and adaptations to the core-agent programme were required. Ultimately, the simulation environment and its interfaces should be as close to reality as possible, such that no modifications to the code are necessary prior to implementation in a UAV system. This chapter describes each 
environment, outlines the development steps that were followed, and provides details on the tests that were performed along with their results. Based on the lessons learned during this work, a way forward for further development is also presented. Central to the latter two simulation environments is an interface with X-Plane.

\subsection{JAVA-Based Simulation Environment}

The decision was made to develop a simulation environment that ran entirely in a common language, rather than trying to initially interface the JACK program with $\mathrm{X}$ Plane. Two options were available. The first involved using another product from Agent Oriented Software, called JACK Sim, while the second involved using custom developed JAVA windows. Instead of learning a new piece of software (JACK Sim), the decision was made to take advantage of JACK's ability to call external JAVA routines.

While the JACK software documentation claims that UDP message formats are used for sending information between agents and with other JAVA programmes, the software actually wraps a series of characters at the beginning and end of each message. Before the external JAVA routines can handle the UDP messages from JACK it is necessary to remove the extra layer of wrapped characters.

The first step in the JAVA-based environment is the setup of a client/server interface between the JACK agents that either require inputs or post outputs. The client is also used to remove the extra characters from the UDP message. UDP messages are sent from JACK to the external devices such as the ATR algorithm, autopilot or GCS, after being 
routed through the JAVA client. Similarly, UDP trigger messages required by the feedback, pilot and ATR agents are routed through the JAVA server.

Three JAVA routines presently run on the same computer as the JACK software, and they communicate through ports 1950 (GCS), 2950 (autopilot), and 3950 (ATR). Before the core-JACK programme runs, it must first establish connections with these routines. Once the JACKCLIENT (Figure 7-1) confirms that all three connections have been made, the JACK programme is executed.

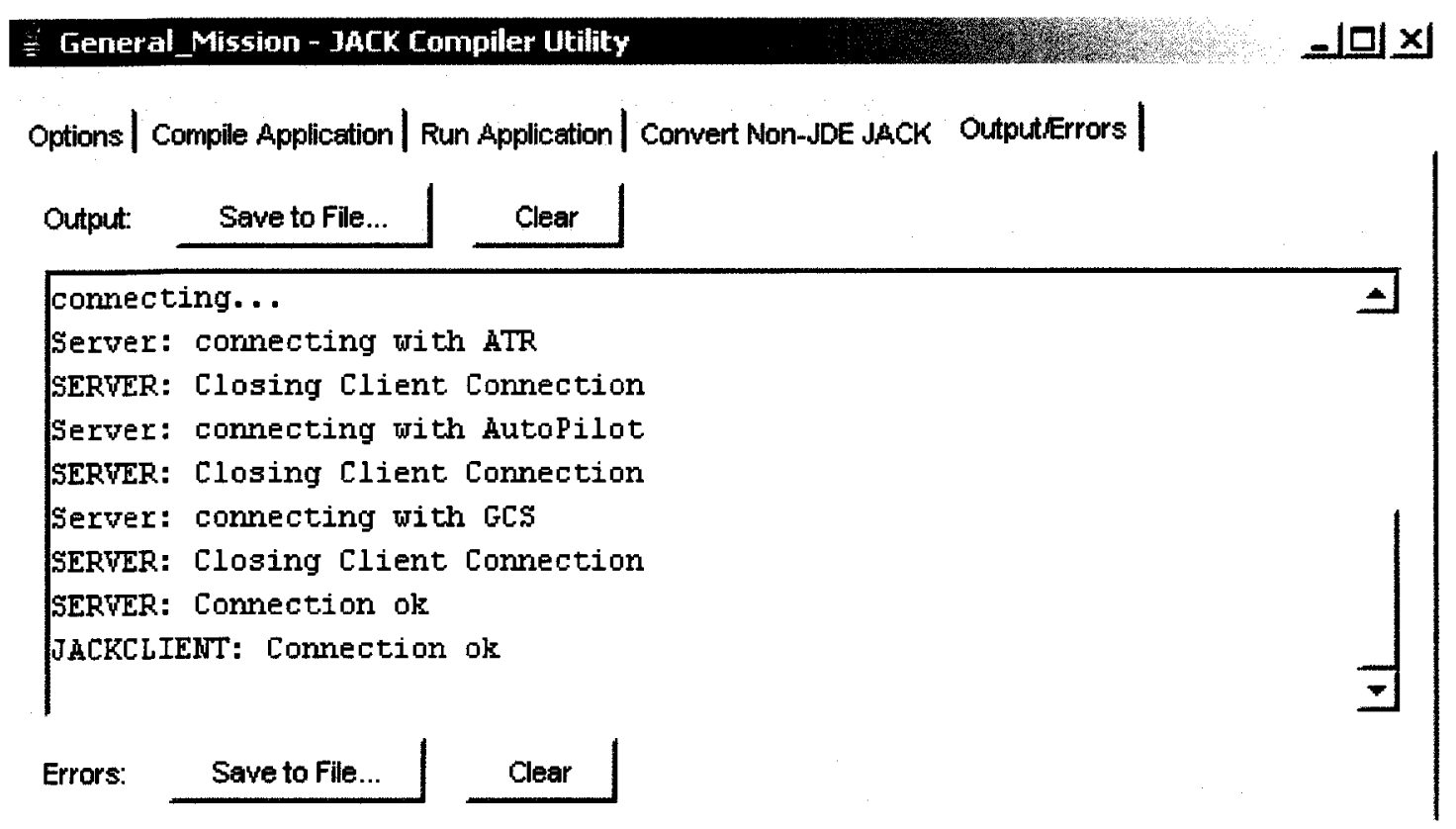

Figure 7-1: Server connection confirmation

\subsubsection{Autopilot Model}

The autopilot model begins by reading and storing the waypoints that are provided in a text file, prior to executing the JACK program. Without having constant feedback for the 
aircraft position and proximity to the next waypoints, the waypoints must be toggled through a JAVA-window representing the autopilot system. A human operator must toggle through each waypoint manually in order to prevent JACK from reading the waypoint file and executing the entire mission in a matter of seconds. Toggling is done by inputting three integer values that are separated by commas. The autopilot routine then sends messages to the autopilot and feedback agents in the following format $<\mathrm{G}^{*} 1|1| 1>$. The values are simply placeholders which are acting to inform the agents that a waypoint has been reached. It is important to note that waypoints which are displayed on the autopilot window are always one step ahead of those shown on the GCS window (described in Section 7.1.2). The points shown on the autopilot window indicate the current destination for the UAV.

\subsubsection{GCS Model}

The GCS window provides visual cues to an operator, showing where the vehicle has already travelled. As the mission is completed, the path of the vehicle and the location of targets are shown in the GCS Simulation screen. It is also the rudimentary user interface for the operator to select the order in which to re-investigate the medium probability targets. When an operator selects the order, the data format takes the form $\left\langle\mathrm{P}^{*} \mathrm{X} \mid \mathrm{Y}\right\rangle$. When multiple targets are located within the search area, the string has the following format $<\mathrm{P}^{*} \mathrm{X}_{1}\left|\mathrm{Y}_{1}\right| \mathrm{X}_{2} \mid \mathrm{Y}_{2}>$. Figure 7-2 shows a completed mission following the investigation of one high probability and one medium probability target. Starting from the bottom left the parallel track search pattern is used. When the high probability target was located, the closer look algorithm created a racetrack pattern of waypoints relative to 
the position of the vehicle when a target was spotted. After completing the closer look pattern, the vehicle continued with the original search pattern and later detected on medium probability target. Once the search pattern had been completed, the GCS operator selected the location of the medium probability target, and the UAV proceeded to investigate this location before returning to the origin.

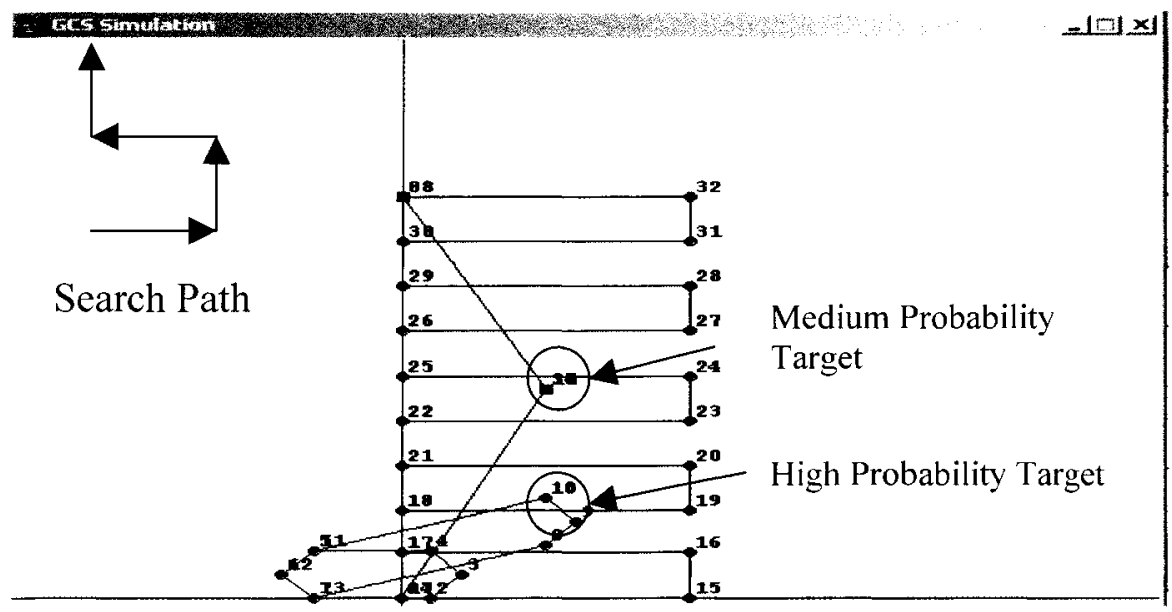

Figure 7-2: GCS simulation window

\subsubsection{ATR Model}

Rather than using the actual ATR algorithm which requires a complex simulation environment (see Section 7.3), a substitute model was generated in JAVA. The JAVA model is simply a graphical user interface that permits a human operator to input the coordinates of a target, and an associated confidence value, as shown in Figure 7-3. The information collected through this window is formatted in the same manner as if it were from the actual ATR algorithm before it is presented to the JACK programme. 


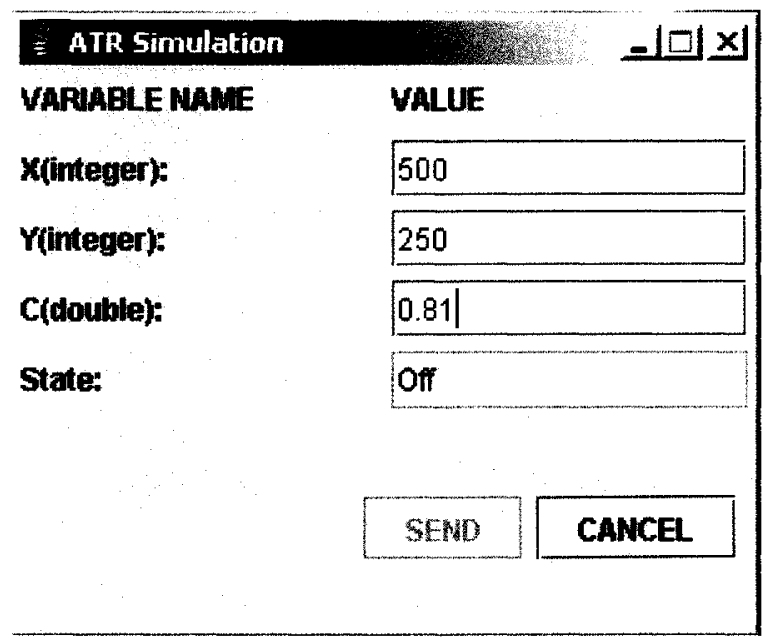

Figure 7-3: JAVA ATR window

\subsubsection{Environment Summary}

During the initial development of the JACK programme, an $x-y-z$ grid system, measured in metres, is used, where waypoint locations are measured relative to the origin point $(0,0,0)$. As such, the inputs to the ATR include target locations measured in metres in the horizontal and vertical directions, while waypoints sent to the autopilot use the same format and also include altitude in metres above ground level.

This fully functional simulation environment is shown in Figure 7-4, where the central block represents the intelligent agent software found both within the air vehicle $(\mathrm{A} / \mathrm{V}$ JACK) and within the ground control station (GCS JACK). It is important to recall that the work described in this section does not include information that is sent to, or received from X-Plane, but rather deals only with the JAVA related components which are shown in the shaded blocks. 


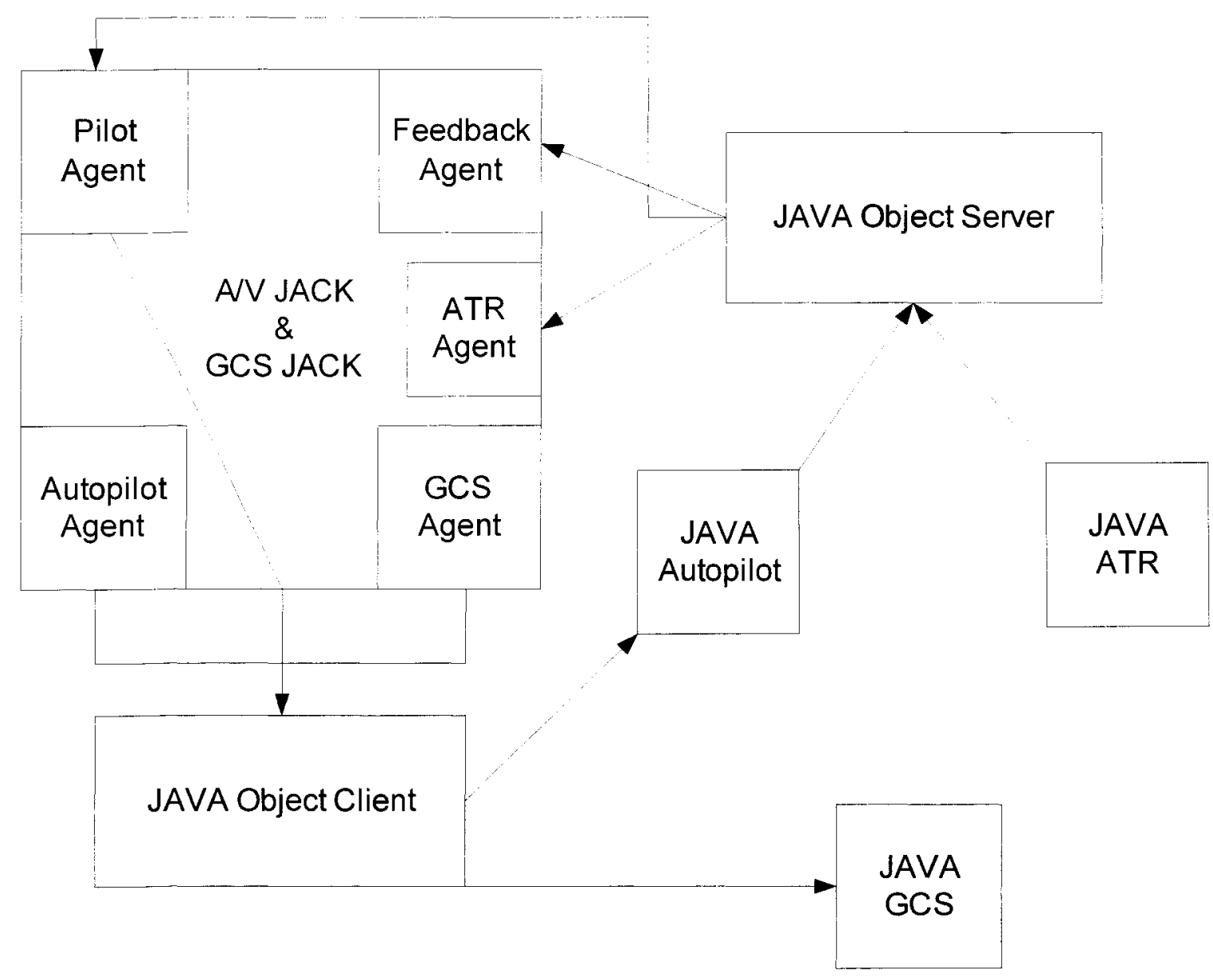

Figure 7-4: JAVA client/server interface

\subsubsection{Simulation Results}

Test results show two major limitations of this environment. Because there is no positional feedback to the agents between reaching waypoints, it appears that the feedback and autopilot agents substitute the origin for the vehicle's current position at any point in time. This problem was discovered when a high probability target was located in the centre of the search area, and the path on the GCS showed the vehicle first returning to the origin. The closer look pattern uses a series of points that are located relative to each other. When the closer look program executed as it was intended, rather than taking its current position between two waypoints, it reverted to $(0,0,0)$ before 
executing the turns and descent. By the time the vehicle was lined up over the position of the target, the aircraft had descended to $-150 \mathrm{~m}$ AGL according to the output displayed by the JACK runtime window. Despite this minor error caused by the simulator environment, the JACK program functioned properly. Due to the way the program was developed it was determined that leaving the altitude obtained by the feedback agent as an undefined value, thus returning a value of zero when called, was a better solution over hard-coding a value for the purposes of this simulation.

A second problem was discovered during testing that is related to the processing of targets. Because an operator has to input the target information through the JAVA ATR window, it is possible to place targets which would not be seen during the current swath of the sensor. Rather than throwing an exception error or ignoring this information, the JACK program immediately investigated the high-probability target. While this practice is consistent with manned search and rescue procedures, it is impossible to spot a distant target when the UAV's only sensor is fixed and pointed downwards. This issue can only be resolved when the ATR is connected to the aircraft autopilot system, to ensure it only reports targets that are within the sensor's current footprint.

\subsubsection{Lessons Learned and Wav Forward}

During the testing of this simulation environment it became clear that the core JACK program does work properly. Having identified some inherent positioning limitations associated with the JAVA setup, it was possible to validate that the JACK program does take appropriate action depending on the target positions and confidence value from the 
ATR. Furthermore, this setup demonstrates that the JACK development environment is able to call and communicate with other JAVA programs and that the JAVA-based client/server program is an effective way to pass information to and from the JACK program. This setup proved to be the key element in the development of the second simulation environment.

\subsection{C++ Based Test Environment}

Following the lessons learned from the developments of the JAVA-based simulation environment, an independent method was developed to provide a means of visualizing the results made by the JACK software. This second simulation environment aimed at connecting the JACK software to the X-Plane flight simulator programme. The same JAVA client/server described in Section 7.1 passes data between JACK and two intermediary translator programs described in Sections 7.2.1 and 7.2.2, which were developed in $\mathrm{C}++$ to interact with X-Plane. A high-level overview of the simulation environment is provided in Figure 7-5.

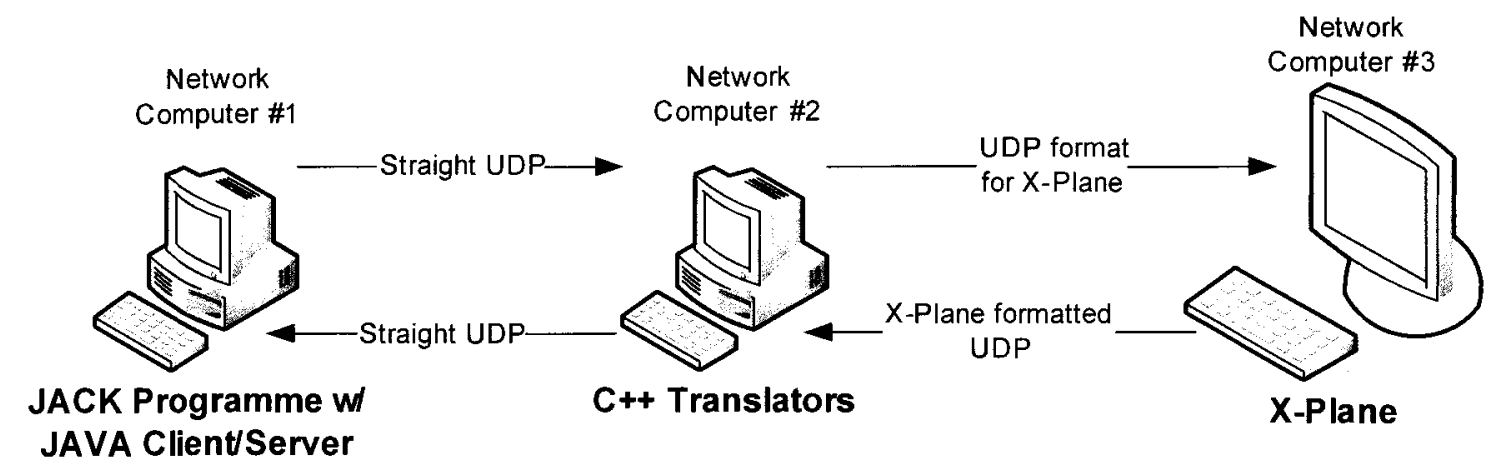

Figure 7-5: Simulation environment hardware configuration 
Here the translators either publish or subscribe on a specific port and computer IP address. This setup is consistent with an actual air vehicle where an internal local area network exists. While UAV autopilots take latitude and longitude positions for waypoint information, X-Plane's autopilot system typically flies by headings, beacons, or markers. However, the ability to fly by GPS points also exists within X-Plane's flight management system. Using a software plug-in, described in Section 7.2.1, an interface to the flight management system is possible. With this addition, X-Plane's autopilot is controllable in a manner that is similar to the autopilot found in UAVs.

\subsubsection{X-Plane Translator Programs}

The X-plane program is used both as a visualization tool and for modelling flight and sensor dynamics. As a visualization tool, it can be programmed to fly over the actual terrain found in the area around Lesser Slave Lake, and therefore provide appropriate feedback for observing the UAV's behaviour. Another use of X-Plane is as a flight and sensor simulator. In this mode it is possible to design a custom UAV that will include different handling properties and avionics or to simulate the autopilot system onboard a UAV. X-Plane can also generate failures to the UAV system such as system failures, atmospheric effects, or diminished aircraft performance.

The X-Plane autopilot is capable of receiving commands and the program can output details about the air vehicle's flight behaviour to other computers. X-Plane is a resourceintensive application and as such, is installed on a dedicated Pentium 4 computer with a 3.0GHz clock, 2GB of RAM, plus $256 \mathrm{MB}$ of video RAM. One major challenge of 
working with X-Plane is the lack of formal documentation regarding the format of data communications. Furthermore, between releases of the software the data field changes have occurred. As others have found (McManus, 2003) the undocumented and changing input/output fields make it difficult to design a generic interface. All work described in this chapter uses X-Plane version 7.63. Table 17 outlines location of key data fields.

Table 17: X-plane Data Communications

\begin{tabular}{|l|l|l|l|}
\hline \multicolumn{2}{|c|}{ Input to X-Plane } & \multicolumn{2}{c|}{ Output from X-Plane } \\
\hline Waypoints (lat/lon) & $\begin{array}{l}\text { Through } \\
\text { FMS }\end{array}$ & $\begin{array}{l}\text { Current Position } \\
\text { (lat/lon) }\end{array}$ & Index 18, col. 1 \& 2 \\
\hline Altitude & $\begin{array}{l}\text { Index 102, } \\
\text { col. 4 }\end{array}$ & $\begin{array}{l}\text { Current Altitude (ASL } \\
\text { or AGL) }\end{array}$ & Index 18, col. 3 or 4 \\
\hline Speed & $\begin{array}{l}\text { Index 102, } \\
\text { col. 1 }\end{array}$ & Current Velocity & Index 02, col. 1 \\
\hline
\end{tabular}

As the aircraft moves in X-Plane, vehicle information is updated 15 times per second and is organized in a number of indexes within the program. Each translator block is set up to subscribe ('listen') or publish ('send') information to these indexes. After establishing the format of each index within the $\mathrm{C}++$ header files of the 'send' and 'listen' translators, individual column values are then read. As an example, the format of 'Index 18: aircraft positions' is shown in Table 12. There are eight columns for each index; however, some columns such as V4 and V5 do not contain any information, resulting in their values being set to -999 (see Table 18).

In most cases the information required by JACK for the feedback agent can be obtained through the indexes of data, similar to that shown in Table 18. Unfortunately, while current vehicle position information is available from Index 18 , it is not possible for the 
autopilot agent to send waypoint information to the autopilot system through the same index. Instead, a separate program is required to interact with the flight management system (FMS), which internally communicates with the autopilot system.

Table 18: X-Plane Index Formats

\begin{tabular}{|c|c|l|l|}
\hline Content & $\begin{array}{c}\text { \# of } \\
\text { Bytes }\end{array}$ & \multicolumn{1}{|c|}{ Example } & \multicolumn{1}{|c|}{ Comments } \\
\hline "DATA" & 4 & DATA & $\begin{array}{l}\text { Identifies that the packet contains data } \\
\text { information }\end{array}$ \\
\hline 0 & 1 & 0 & Byte zero \\
\hline $\begin{array}{c}\text { '0' if PC or } \\
\text { '1' if Mac }\end{array}$ & 0 & 0 & This '0' indicates that a PC is used \\
\hline Index & 4 & Index18 & Index 18 identifies the aircraft position \\
\hline V0 & 4 & float RefLat & Plane latitude \\
\hline V1 & 4 & float RefLon & Plane longitude \\
\hline V2 & 4 & float AltFmsl & Altitude (feet) relative to mean sea level \\
\hline V3 & 4 & float AltFagl & Altitude (feet) above ground level \\
\hline V4 & 4 & float empty & No data in this field (set to -999) \\
\hline V5 & 4 & float empty2 & No data in this field (set to -999) \\
\hline V6 & 4 & float LatSouth & Southern latitudes are negative \\
\hline V7 & 4 & float LonWest & Western longitudes are negative \\
\hline $\begin{array}{c}\text { '0' if PC or } \\
\text { '1' if Mac }\end{array}$ & 0 & 0 & Last byte \\
\hline
\end{tabular}

\subsubsection{Flight Management Systems Plug-in}

In X-Plane, the Flight Management System (FMS) is separate from the autopilot which can be controlled through UDP messages. Instead, the FMS uses an on-screen interface, where a human pilot sets radio beacon locations or GPS coordinates based on the desired route. Within a UAV, waypoint (lat/lon) information is used to control the aircraft position. A custom plug-in for X-Plane was developed by S. Barbour following personal communications with the author (21 Jan 2005). Barbour is one of the software programmers who developed the X-Plane software developer's kit that enables users to 
create custom plug-ins. While the FMS uses code which is internal to X-Plane, the open source plug-in permits the same functionality. Though the plug-in was developed to provide a graphical user interface to the FMS, it is presently hard-coded with GPS coordinates to act as start and end-points for flight line segments.

\subsubsection{Environment Summary}

With X-Plane able to handle GPS waypoint information, and having a translator for inputting and extracting information from the programme, the setup works well when the data is hard-coded into the program. However, a real-time solution is required. This is where the client/server interface from the JAVA simulation environment is used. By modifying the translators and the FMS plug-in to include both a loop and defined socket value, it is possible to link the client/server with the translators. Doing so allows the JACK software to update the values within each translator, instead of hard-coding each number. Presently, the translator program responsible for sending altitude and velocity information to X-Plane, functions properly. Similarly, the translator program responsible for listening to vehicle position (lat/lon), altitude and speed has also been tested and functions properly, as does the FMS plug-in when data is hard-coded by a user.

Future work will require creating the real-time interface between the JAVA client / server, the two translator blocks and the FMS plug-in using an interface with the sockets. Figure 7-6 shows this simulation environment in its tested state. The heavyweight lines show connections that work, while the dotted lines indicate areas that require further development. 


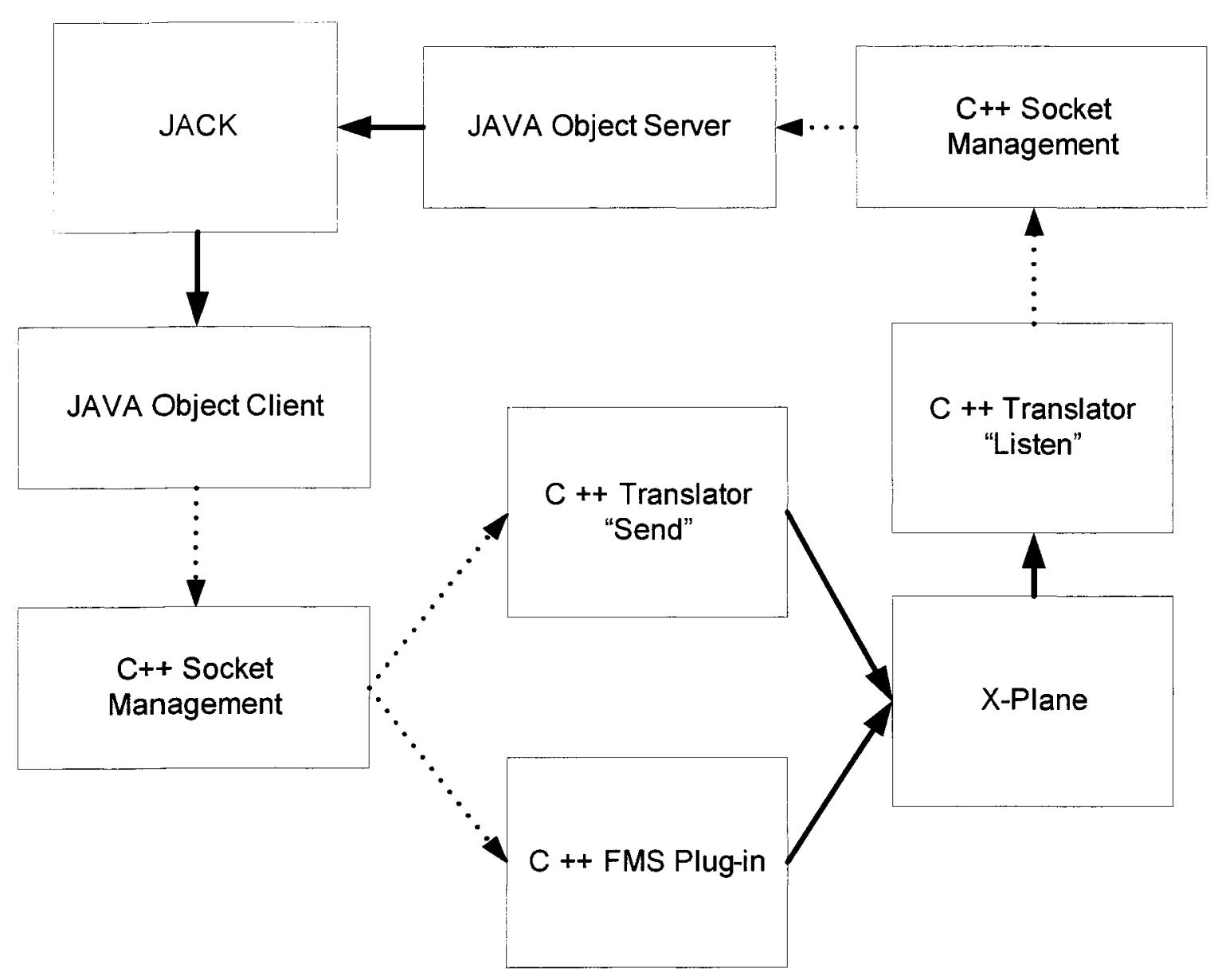

Figure 7-6: $\mathrm{C}++$ Based simulation environment

\subsubsection{Required Modifications to JACK}

As mentioned in Section 7.1.4, during the early development of the JACK program, waypoint information was measured in metres rather than degrees of latitude and longitude. However, the use of metres will not work when connected with X-Plane, which requires waypoint information in degrees of latitude and longitude. The changes that are required pose additional challenges to the development of the agent's closer look algorithm, because the conversion from metres to degrees of latitude and longitude does not follow a linear relationship. Appendix $\mathrm{C}$ describes and highlights the importance of 
this difference. By selecting a reference meridian and a parallel for flight operations, it is possible to calculate the appropriate conversion factor of metres per degree of latitude and longitude. For demonstration purposes the approximate location of Slave Lake is $55.2^{\circ} \mathrm{N}, 114.4^{\circ} \mathrm{W}$. Alternative mapping systems such as the Universal Transverse Mercator (UTM) grid provide a flat mapping, thereby eliminating this problem.

\subsubsection{Simulation Results}

Testing of the translator enabled simulation environment had two objectives; to better understand how X-Plane works, and to properly connect X-Plane with JACK. In order to meet the first objective, numerous flights were flown either manually or under control of the X-Plane autopilot system. By reading the post-flight data logs for selected indexes, column values were determined and recorded. Following this exercise, the internal structure of the translator blocks changed.

Experimental flights demonstrated that X-Plane has difficulty following closely spaced tracks. The autopilot system does not create spline curves between waypoints, and instead reads one point at a time. It appears that the aircraft has problems making small radius $(R=112 \mathrm{~m})$ turns between the end of one survey line and the beginning of another while flying at $70 \mathrm{kts}$. By experimenting with different sets of waypoint files it was found that the best results are obtained when a maximum bank angle was provided along with the end waypoint for each search path. 
Using initial plans that provided start and end waypoint information, the aircraft made very wide turns between the end of one path and the beginning of another. Incidentally, X-Plane uses the same mathematics as described by Equations 5-9 of Section 6.2.3.5.1.1, to calculate turning radius. When only end points were used, and a maximum bank angle of $20^{\circ}$ was provided, the aircraft executed the intended pattern more precisely.

\subsubsection{Lessons Learned and Wav Forward}

Results of the flight path for closely spaced waypoints are discouraging because they show that the aircraft model in X-Plane is more likely to fly the intended path when only a select number of waypoints are defined. Figure 7-7a shows a series of waypoints defined by the original search pattern, while Figure $7-7 \mathrm{~b}$ shows the pattern used within X-Plane to obtain the same desired results.

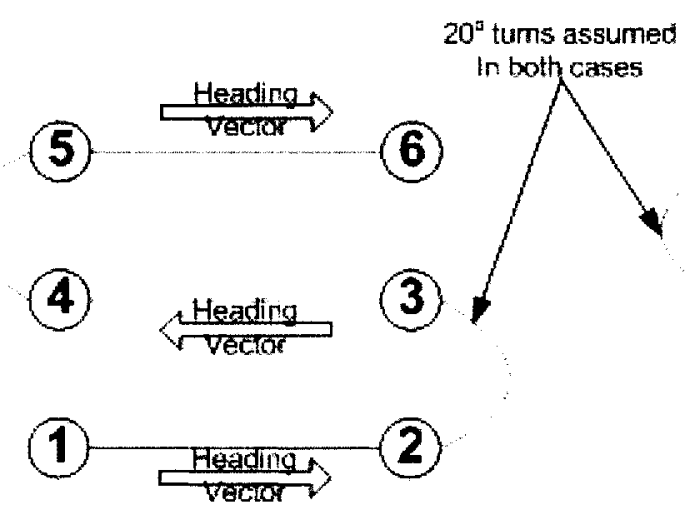

(a)

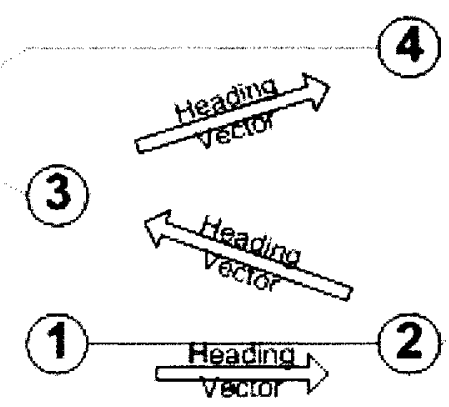

(b)

Figure 7-7: Closely spaced waypoints and X-Plane

In the case shown in Figure 7-7b, it difficult for the JACK program to calculate its current heading while passing over a given target, because the vector between waypoints 
is not the same as the followed path. This result has more to do with X-Plane's autopilot system than it does with one that might be found within an air vehicle.

Complete testing of an X-Plane aircraft under the control of JACK still requires further development. With the initial successes demonstrated by the individual translator blocks, along with a more comprehensive understanding of how X-Plane handles waypoint information, future development of the simulation environment must overcome these shortcomings.

Additional modifications to the core-JACK program are also required to enable a user to select any location in the world by latitude and longitude. Using a look-up table as an example, a user could then perform the appropriate conversion between metres and degrees for the closer look plan which uses waypoints that are measured relative to each other.

\subsection{HLA-Based Simulation Environment}

A third alternative simulation environment was also explored. In this simulation environment the high level architecture (HLA) is used to directly connect JACK to XPlane and eventually the actual ATR algorithm. The high level architecture is middleware software that was developed by the US military for the construction of simulation architectures, where the HLA provides a mechanism for operating multiple simulators together. Provided each component (JACK, X-Plane, ATR) has been developed to work 
in an HLA environment, it is possible to add or remove a component without affecting the stability of the simulation.

\subsubsection{HLA Basic Elements}

Each simulation application running in the HLA is called a "federate", while a set of federates that are running together with a common purpose or goal is called a "federation". A federate can connect to and instruct the run time infrastructure (RTI), which then can create a federation. While the federation is running, ownership of data along with the data publication or subscription policies are defined by the Federation Object Model (FOM). The FOM itself is set up according to the simulation designer's own decisions about the structure of the intended architecture. Upon completion of the common goal, each federate resigns from the federation, and when the last federate resigns the RTI destroys the federation.

At least three different RTIs are known to this author. All three are completely independent and do not work together due to their internal designs. Therefore any simulation environment must be constructed using only one RTI implementation. The original RTI was developed by the Defence Modeling and Simulation Office of the United States (DMSO). Following the recommendations of other HLA users at Carleton University and within DRDC-Valcartier this project uses the well-documented DMSO RTI 1.3NGv6. Recently the Institute of Electrical and Electronics Engineers (IEEE) adopted and modified the DMSO RTI and created their own standard, known as RTI 1516. While HLA $1.3 \mathrm{NG}$ is typically a single-threaded environment, HLA 1516 is fully 
multi-threaded, similar to JACK. Following discussions between the author and D. Thibault of DRDC-Valcartier (personal communications, 11 Feb 2005) JACK's internal thread handling is capable of working with either RTI $1.3 \mathrm{NG}$ or RTI 1516. The third type of RTI was developed by MäK, an American company, and uses yet another set of libraries and functions. For each type of RTI, there is also a software interface layer. Because JACK is an extension of JAVA, the JAVA-bindings layer is required. With this basic understanding of the main elements involved in an HLA environment it is possible to explore if the JACK program can be modified to operate in this environment.

\subsubsection{JACK to HLA}

The ability to send messages between JACK agents and other software entities through the HLA was recently developed at DRDC-Valcartier where a JACK capability can be added to existing agents. Two capabilities were created to facilitate operation within either RTI 1.3NG or RTI 1516. In order for the complete simulation environment to work, the HLA capability must be added to the ATR, Autopilot, and Feedback agents. This would enable the JACK software to communicate with other entities within the HLA environment. However, each JACK plan that publishes or subscribes to external data must be re-written, and this will depend on the Federation Object Model policies that are enforced by the RTI. 


\subsubsection{Setting up the Environment}

While an HLA-based simulation environment would provide an elegant solution to the simulation needs, it also introduces a plethora of potential problems. This section proposes eight steps for setting up an HLA-based simulation environment.

1. Install the DMSO RTI $1.3 \mathrm{NG}$ v6 for Windows 2000 (there is also a version for NT). It is important to follow the installation manual precisely. Environmental variables must be set properly, as any deviation from the installation manual will result in the program failing to install properly.

2. Install the JAVA bindings for RTI $1.3 \mathrm{NG} v 6$ in the same directory as the files that were installed for step 1.

3. Before proceeding, compile and execute the HelloWorld code for both the $\mathrm{C}++$ and JAVA version to verify that the RTI and the JAVA bindings installed properly.

4. Within JACK, compile and execute the capability that communicates with the RTI. For this to work properly, the RTI must be running. By executing this as a stand-alone application, it is possible to verify that everything works properly. 
5. Import the capability into the core-JACK program and add it to the ATR, Feedback and Autopilot agents. Rather than removing the JAVA-based windows that were previously developed, augment the system by adding new plans.

6. Add an HLA-based window (dummy federate) that simply displays the passage of data that is being published by the JACK program. A similar application should be used to provide dummy data to JACK, to ensure that the subscription plans are functioning properly. This interim step is necessary and will confirm that the JACK to HLA portion is working properly, before introducing X-Plane to the system.

7. Connect X-Plane to the HLA environment. While an HLA plug-in has been developed by DRDC-Ottawa's FFSE, it was written for the MÄK RTI, which is incompatible with the DMSO RTI. The current plug-in calls libraries that are internal to the MäK implementation. Therefore, there are two options: either identify the functionality of the elements that are called with the libraries and find the similar version in the DMSO libraries, or re-write the plug-in for the DMSO RTI. The latter is likely the better choice.

8. At this point all the individual components have been tested independently. It is now an appropriate time to connect both JACK and X-Plane to the RTI while keeping the intermediate dummy federates part of the system as monitors. 


\subsubsection{Lessons Learned and Way Forward}

While steps 1 and 2 were followed, numerous difficulties arose at step 3, and all steps beyond this point have not been attempted. RTI $1.3 \mathrm{NG}$ v6 was originally compiled using $\mathrm{C}++$ version 6 , while the research computer accessed $\mathrm{C}++$ version 7 . It is believed that this is part of the reason that the HelloWorld applications of step 3 does not run. The JAVA bindings were also installed, however for one reason or another the HelloWorld JAVA would not execute. As outlined in the eight-step approach, an incremental development process is required. Only upon successful completion of each step can development proceed, with significant efforts required in steps 5-7. While HLA may provide an elegant solution to the development of a simulation environment, it also requires substantial changes to program plans developed in JACK.

Future efforts should focus on the simulation environment discussed in Section 7.2. The additional code needed to connect the JAVA server/client with the $\mathrm{C}++$ blocks is most likely very modest compared with the numerous lines of code necessary to the setup and test a HLA-based simulation environment. Furthermore, modifications to the coreprogram are also required, as each of the plans used to communicate with the external environment must be re-written for compatibility with the HLA environment. 


\title{
Chapter 8: Conclusions \&
}

\section{Recommendations}

\author{
CHAPTER SUMMARY
}

This chapter discusses five aspects that have been developed in this thesis, including:

- One concept of operation for using UAVs within a Search and Rescue mission,

- The applicability of intelligent agents for the mission-level control of a UAV,

- The implications of using intelligent agents within an air vehicle and ground control station to STANAG 4586,

- The utility of the proposed five-agent arrangement for interfacing with external systems, and

- The exploration of various simulation environments to enable testing.

Recommendations for future work are based on two overall directions and include the expansion of the current multi-agent software programme and further development of the simulation environment. 


\subsection{Conclusions}

This thesis considered the design and development issues of integrating intelligent agent software into an air vehicle system. Through this thesis a concept of operation for using UAVS within a SAR mission has been developed along with the design of an agent-based program. Three simulation environments were also developed to different degrees of completeness in order to test the agent-based program. Intelligent agents also have implications to STANAG 4586 where their use was not considered in the original development of the standard. Contributions to each of these areas are discussed below.

\section{How UAVs might be used within a Search and Rescue mission.}

Through modifications to the 1994 force planning scenarios, one concept of operation for UAVs within a search and rescue mission is developed in this thesis. From the scenario presented in Chapter 3, a notional UAV system including a fixed-position camera, automatic target recognition (ATR) algorithm and autopilot system is coupled with an intelligent agent programme.

By uploading a waypoint file to the multi-agent programme designed in Chapter 6 , the agent program demonstrated that it is capable of executing one of the search patterns defined in the National Search and Rescue Manual. While flying at $300 \mathrm{~m}$ above ground level, the UAV's camera system acquires pictures of the terrain directly beneath the vehicle. As each image is acquired it is processed by the ATR, possible target locations are identified and recorded in databases within the agent programme. Depending on the confidence value provided for each target's position, the intelligent 
agent programme determines what appropriate action should be taken following a set of prescribed plans.

At the completion of the mission, all possible targets identified by the ATR algorithm have been geo-located and photographed from $150 \mathrm{~m}$ above ground level. By only transmitting the low-altitude (high-resolution) images to the ground control station operator, bandwidth requirements are reduced. Therefore, by capitalizing on the UAV's ability to perform long endurance missions in daylight or night conditions, SAR operations could benefit from the addition of autonomous UAVs. Future testing of this concept of operation is necessary to evaluate what impact it will have on the workload of the operators.

\section{The suitability of the proposed five-agent model within a UAV system.}

For an intelligent agent programme to be successfully integrated within a UAV system, it must interface with the existing software and hardware. Using one agent at each interface, between the agent software and the ground control station, ATR algorithm, and autopilot system, proved to be a viable solution within the simulation environment. Using UDP formatted messages over a TCP/IP network, the three interface agents were able to communicate with the other components. This communication feature of the JACK programme is critical for its future integration within a UAV system. With two additional internal agents used to monitor the system and to make decisions, the five agent model proposed in this thesis was able to 
process target information, re-plan waypoints, and control the UAV platform while maintaining operator awareness.

\section{How can various simulation environments be coupled with the JACK} software.

Three simulation environments evolved during this thesis. Decisions made by the intelligent agent programme were successfully visualized within a fully-functional two-dimensional JAVA-based simulation environment. Using JAVA windows which interfaced with the JACK program through unique port numbers and UDP formatted messages, it was possible to both post and pull information from the JACK program. Following the demonstration of a JAVA-based simulation environment, a subsequent $\mathrm{C}++$ and X-Plane based environment was started. With the increased complexity of this environment, many of the individual program elements have been tested and proven to work properly when hard-coded. Future work in this area is still required to enable real-time testing. Similarly, an HLA-based environment was also explored. Development of an HLA environment required both an understanding of the language's unique architecture and the complete re-write of all external JACK events. As a result of these issues and complexities faced while trying to establish the runtime environment, an HLA simulation environment was not created as part of this thesis.

Testing did occur through the JAVA-based simulation environment, and consisted of entering high, medium and low probability targets through the JAVA-based ATR window. These tests showed that the agent program properly handled all values of 
target information. However, without completing any line profiling it is impossible to know whether all lines of code within the agent program were executed during testing. Similarly, concepts of fault tolerance were not considered during either the design or development of the agent-based system. As a result, the solution developed in this thesis requires substantial testing and evaluation before it can be integrated into the high fidelity simulator at DRDC-Ottawa, let alone within an actual air vehicle. Depending on the chosen test environment, several changes to the core agent-program are required.

\section{How intelligent agents impact STANAG 4586}

When intelligent agents are used onboard an air vehicle, it is also necessary to include similar software within the ground control station to receive inter-agent messages. By using two instances of the JACK software, the role of the vehicle specific module as defined in STANAG 4586 is diminished, and the role of the inter-agent messages increased. For digital readings acquired onboard the UAV and recorded by the agents within the UAV, inter-agent messages between the air and the ground station augment the current capabilities of the VSM, and therefore the use of intelligent agents within an air vehicle will require changes to the STANAG.

From this work, it follows that there are several new areas of opportunity exist for further development. These areas are identified and discussed in the following section. 


\subsection{Recommendations for Further Work}

There are two different approaches to advancing the level of autonomy towards the higher levels defined by the autonomous control level chart (OSD, 2002; Clough, 2002) shown in Figure 8-1. To fully demonstrate Level 4 autonomy, the chosen UAV platform must first demonstrate its ability to "pass" the requirements of Level 3: Response to realtime failures. Pursuant to the integration of intelligent agent software developed in this thesis into such a vehicle, the next step is to validate all simulation results through flight testing. Using multiple UAVs, where each individual vehicle demonstrates the capability of onboard route re-planning, one could then introduce another level to the agent program called JACK teams. This additional layer assigns group goals to enable a teaming approach. Demonstration of a system of UAVs working together during a simulation and subsequent flight test would represent a significant step forward.

The second alternative to advancing the level of autonomy is to increase the number of agents and their capabilities within a single vehicle. Real-time power monitoring during different phases of flight, terrain following and obstacle avoidance (path planning) all pose technically challenging problems but have the potential to enable more complex missions. Pursuing the development of agent systems from either the single or multiple vehicle approach poses a unique set of challenges, where future work in these areas could lead to demonstrations of autonomy Levels $5-10$. An incremental approach is advised. 


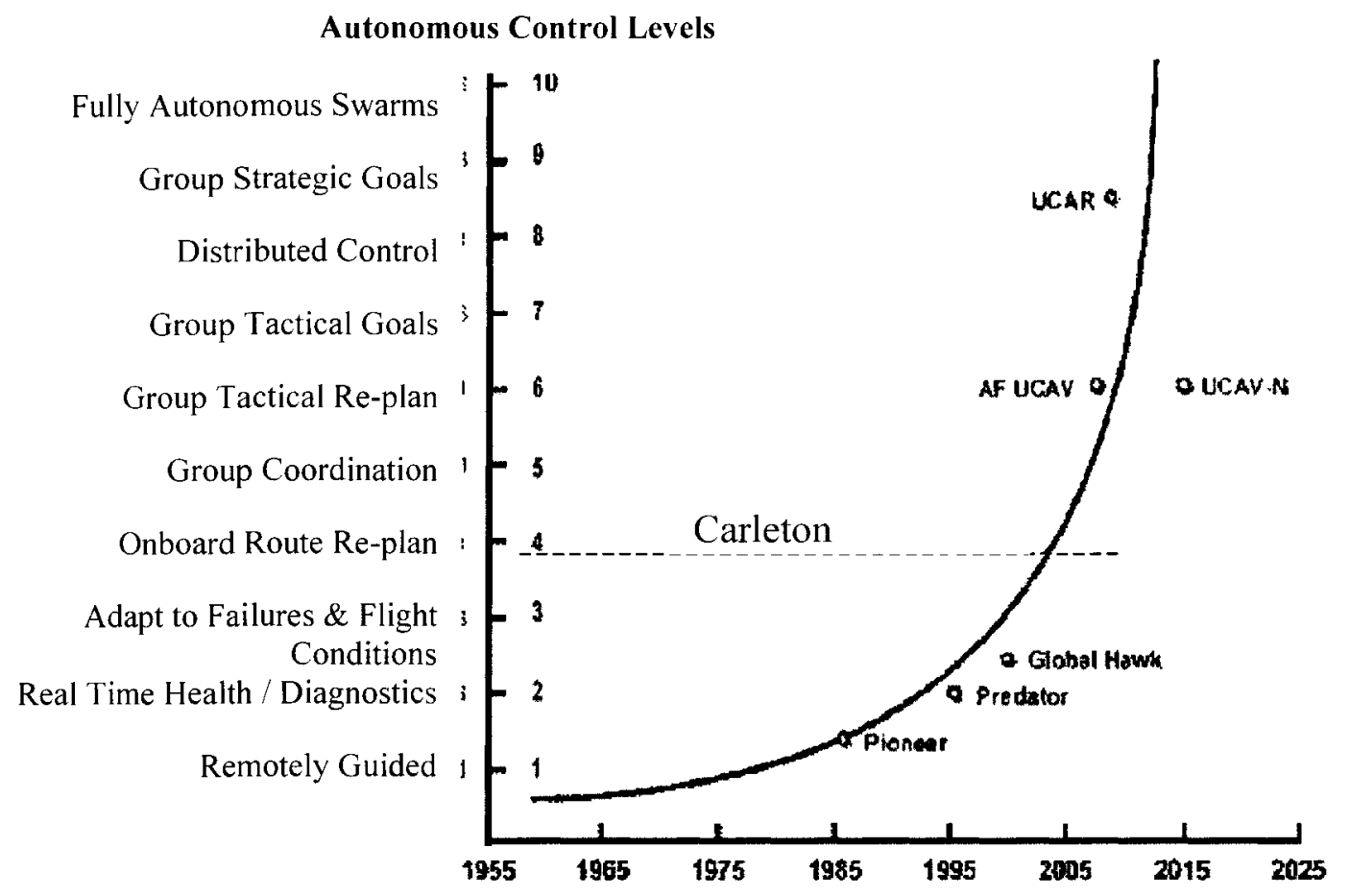

Figure 8-1: Level of autonomy development at Carleton University

Further work in human factors engineering is also warranted to evaluate the impact of intelligent agents on the cognitive workload of UAV operators and image analysts. Other areas of future work should include:

- Develop the HLA simulation environment which would enable JACK to be tested with various sensors, hardware, or software components.

- Further develop the agent software to make better use of the feedback agent and test it under a variety of flight conditions.

- Consider using a different simulation environment rather than X-Plane. One such option is a program called Flight Gear, which is an open source code program with extensive documentation readily available. 
- Once a fully operational simulation is running, it could be used to help assess the impact that intelligent agents have on the cognitive workload of UAV operators and image analysts.

- Install the JACK software on to a PC-104 or similar small target computer, and integrate the system into an air vehicle to conduct follow-on flight testing. A hardware-in-the-loop enabled simulation environment that is coupled with JACK would be a tremendous step forward, prior to airborne tests. Such an environment should include the MicroPilot's MP2028 autopilot system and MicroPilot's Xtender software developer's kit (SDK) for the ground control station software, which includes a six-degree of freedom aircraft model. By using the Xtender SDK coupled with the Horizon GCS software, X-Plane will no longer be required.

With numerous possibilities for future developments, it is expected that multi-agent systems will play a key role in the future of autonomous unmanned aerial vehicle systems especially when more complex agent-architectures are developed. 


\section{References}

Abi-Zeid, I., \& Lamontagne, L. (2003). A functional decomposition model of case prosecution in Joint Rescue Coordination Centers and related projects: Technical Report TR 2000-150. Valcartier, Canada: Defence R\&D Canada.

Abi-Zeid, I., \& Doyon, B. (2003). Using a geographic decision support system to plan search and rescue operations. International Journal of Emergency Management, Vol. l, No. 4, pp. 346-362.

Abi-Zeid, I., \& Frost, J. (2005). SARPlan: A decision support system for Canadian Search and Rescue Operations. European Journal of Operational Research, Vol. 162, No. 3, pp.630-653.

Aerosonde Pty. (2004). DSTO ABSI Trials, Retrieved December 18, 2004, from http://www.aerosonde.com/drawarticle/91

AeroVations Associates, (2003). Priorities for UAV Research and Technology Development in Canada: under contract No. 514325 to the National Research Council Canada, Report No. AVA 04-02. Ottawa, Canada: AeroVations Associates.

Amiran, D.H.K., Schick, A.P. (eds.) (1961). Geographical Conversion Tables, Aschmann and Scheller, Zurich, Switzerland, pp.216.

AOS, (2004). JACK Intelligent Agents User Guide; Development Environment Manual; Design Tool Manual. Melbourne, Australia: Agent Oriented Software Pty Ltd.

Bandura, A. (1990). Conclusion: Reflections on nonability determinants of competence. In R.J. Stenberg \& J. Kolligian Jr. (Eds.), Competence considered (pp. 315-362). New Haven, CT: Yale University Press.

Barber, K., \& Lam, D. (2001). Architecting agents using core competencies: Technical Report TR2001-UTLIPS-AGENTS-04. Austin, TX: The University of Texas at Austin.

Barber, K., Goel, A., Han, D., Kim, J., Lam, D., Liu, T., MacMahon, M., Marin, C., McKay, R. (2003). Infrastructure for Design, Deployment and Experimentation of Distributed Agent-based Systems: The requirements, the technologies, and an example. Autonomous Agents and Multi-Agent Systems, Vol. 7,pp. 49-69.

Bernier, Capt. J. (2004). C-130 Navigator and Search Master - Interview with the author, September 1, 2004, JRCC Trenton, ON, Canada. 
Bond, A.H., \& Glasser, L. (Eds.) (1988). Readings in Distributed Artificial Intelligence. San Mateo, CA: Morgan Kaufmann Publishers Inc.

Bratman, M. (1987). Intention, Plans and Practical Reason. Cambridge, MA: Harvard University Press.

Bresciani, P., Giorgini, P., Giunchiglia, F., Mylopoulos, J., Perini, A., (2002). Tropos: An agent-oriented software development methodology: Technical Report DIT-02-0015. Trento, Italy: University of Trento, Department of Information and Communication Technology.

Brule, J.F. (1985). Fuzzy Systems - A tutorial, Retrieved Feb 26, 2005, from

http://itla.edu.do/mv/sources/tutoriales/fuzzy/tutor.htm

Burrafato, P., \& Cossentino, M. (2002). Designing a multi-agent solution for a bookstore with the PASSI methodology. In proceeding of the Fourth International BiConference Workshop on Agent-Oriented Information Systems (AOIS-2002). Toronto, Canada.

Butterworth-Hayes, P., \& van Blyenburgh P., (Eds.), (2004). UAVs: A Global Perspective. London: UVS International.

CASA (2002). Attack of the drones, Flight Safety Australia, Nov/Dec 2002, pp.56-58.

Carryer, A. (2004). Carleton University Avionics Test Bed - Ground Control Station Evaluation, Prepared for DND under contract W7714-030749/001/SV: UAV Systems and Autonomy, 30 January 2004.

Carver, C.S., \& Scheier, M.F. (1998). On the self-regulation of behavior. Cambridge, United Kingdom: Cambridge University Press.

Champagne, L., Carl, G., Hill, R. (2003). Search Theory, Agent-Based Simulation, And U-Boats in the Bay of Biscay, In proceedings 2003 Winter Simulation Conference: New Orleans, LA, Vol. 1, pp 991-998.

Chief of the Defence Staff (1982). Spotters Guide: A-GA-050-016/AG-000, Ottawa, Canada, Department of National Defence.

Chief of Defence Staff, (1994). 1994 White Paper on Defence - Search and Rescue in Canada Scenario. Ottawa, Canada: Department of National Defence Policy Group, Defence Planning and Management.

Clough, B.T. (2002). Metrics, Schmetrics! How The Heck Do You Determine A UAV's Autonomy Anyway? In proceedings of the Performance Metrics for Intelligent Systems Workshop, Gaithersburg, MD. 
Cossentino, M., Potts, C., (2002). A CASE tool supported methodology for the design of multi-agent systems. In Proceedings of the International Conference on Software Engineering Research and Practice (SERP'02), Las Vegas, $N V$.

Dai, L, Carryer, A. (2005). Intelligent Agent Control of an Unmanned Aerial Vehicle: Internal Document, Dept. Mechanical and Aerospace Engineering, Carleton University.

DeLoach, S.A., Wood, M.F., \& Sparkman C.H. (2001). Multiagent systems engineering, International Journal of Software Engineering and Knowledge Engineering, Vol.11, No. 3, pp. 231-258.

Department of Defense (1989). AF Manual 51-40 (C2) Air Navigation; Section 23-4, Departments of the Air Force and Navy, Washington, USA.

Department of Defense (2001). Systems Engineering Fundamentals, Defense Acquisition University Press, Fort Belvoir, VA: US Department of Defense, Systems Management College.

Directorate of Flight Safety, (2004). Aircraft Occurrence Summary: TUAV CU161005, 19 January 2004, Kabul Afghanistan, Ottawa, Canada, Department of National Defence.

Doran, J.E., Franklin, S., Jennings, N.R., \& Norman, T.J. (1997). On Cooperation in Multi-Agent Systems: Report from panel discussion at the $1^{\text {st }}$ UK Workshop on Foundations of Multi-Agent Systems, The Knowledge Engineering Review, Vol. 12, Issue 3.

Durfee, E., Kenny, P., \& Kluge, K. (1998). Integrated Pre-mission Planning and Execution for Unmanned Ground Vehicles. Autonomous Robots, Vol. 5, pp. 97-110.

DRDC Valcartier (2002). Instructions for using SARPlan v2. Valcartier, Canada: Defence Research and Development Canada.

European Association of Geoscientists and Engineers, (Feb 2005). A little goes a long way with Fugro's latest unmanned aeromagnetic survey flying machine, First Break, Vol. 23, pp. 9-11.

Fahstrom, P., \& Gleason, T. (2002). Introduction to UAV Systems. Columbia, MD: UAV Systems Inc.

Ferber, J. (1999). Multi-Agent Systems: An introduction to distributed artificial intelligence. New York: Addison Wesley Longman Inc.

Frost \& Sullivan, (2001). Corporate Market Report \# 7884-16: World Unmanned Aerial Vehicle (UAV) System Markets. 
Georgeff, M., Pell, B., Pollack, M., Tambe, M., \& Wooldridge, M. (Eds.) (1999). The Belief-Desire-Intention Model of Agency. Intelligent Agents $V$, Springer-Verlag Lecture Notes in AI, Vol. 1365.

Hypher, R.P. (1979). Search and Rescue in Canada. In proceedings of a NATO Advanced Research Institute on Search Theory and Applications, Praia Da Rocha, Portugal, pp.73-85.

Jennings, N. (1999). On agent-based software engineering. Artificial Intelligence, Vol. 117, pp. 277-296.

Johnsen, F.A. \& Brown, A. (2003). Detect-See-and Avoid. The X-Press, Vol. 45, No. 3, Edwards, CA: NASA Dryden Flight Research Center.

Johnson, J. (1958). Analysis of image Systems. In proceedings of Image Intensifier Symposium: US Army Engineering Research and Development Laboratory, pp. 100110.

Koopman, B. (1980). Search and Screening. New York: Pergamon Press Inc.

Locke, E.A. (1968). Toward a theory of task motivation and incentives. Organization Behavior and Human Performance, Vol. 3, pp. 157-189.

Locke, E. A., Shaw, K.N., Saari, L.M., \& Latham, G.P. (1981). Goal setting and task performance: 1969-1980. Psychological Bulletin, Vol. 90, pp. 125-152.

Lucas, A., \& Goss, S. (1999). The Potential for Intelligent Software Agents in Defence Simulation. In Proceedings Information Decision and Control '99, Adelaide, Australia.

Lucas, A., Rönnquist, R., Ljungber, M., Howden, N., Corke, P., \& Sikka, P. (2003). Teamed UAVs - A new Approach with Intelligent Agents. In Proceedings AIAA conference Unmanned Unlimited, San Diego, CA.

McManus, I.A., Greer, G.G., Walker, R.A. (2003). UAV Avionics 'Hardware in the Loop' Simulator. In Proceedings $14^{\text {th }}$ Australian International Aerospace Congress, Brisbane, Australia.

Mattson, R.J. (1979). Overland Search for Missing Aircraft and Persons. In Proceedings of NATO Advanced Research Institute on Search Theory and Applications, Praia Da Rocha, Portugal, pp. 87-97.

Miller, G.A., Galanter, E.H., \& Pribram, K.H. (1960). Plans and the structure of behavior. New York: Holt, Rinehart \& Winston.

Minsky, \& Papert (1969). Perceptrons, An introduction to computational geometry. Boston, MA: The MIT press. 
National Search and Rescue Manual (NSM) (1998), Document B-GA-209-001 / FP-001, DFO05449. Ottawa: Canada: Canadian Department of National Defence.

National Search and Rescue Secretariat (NSS) (2004). Search and Rescue Literature, Retrieved June 3, 2004, from http://www.nss.gc.ca

NATO, (1979). Search and Rescue Working Group Report. In proceedings of a NATO Advanced Research Institute on Search Theory and Applications, Praia Da Rocha, Portugal, pp.45-71.

NATO, (1999). RTO-EN-009: Development and Operation of UAVs for Military and Civil Applications. NATO Research and Technology Organization.

NATO, (2002). RTO-ENP-025: Applications, Concepts and Technologies for Future Tactical UAVs. NATO Research and Technology Organization.

NIMA, (1997). Department of Defense World Geodetic System 1984, Its Definition and Relationships with Local Geodetic Systems, National Imagery and Mapping Agency Technical Report 8350.2, Third Edition, UNCLASSIFIED.

Norling, E. (2004). Folk Psychology for Human Modeling: Extending the BDI Paradigm in Proceedings of AAMAS'04, New York.

Office of the Secretary of Defense, (2002). Unmanned Aerial Vehicles Roadmap 20022027, Acquisition, Technology and Logistics, Air Warfare. Washington: US Department of Defense.

Padgham, L., \& Winikoff, M. (2004). Developing Intelligent Agent Systems: A Practical Guide. Chichester, Great Britain: John Wiley \& Sons.

Park, A., \& Williams, W. (2004). Press release: First Flight - True UAV Autonomy At Last. Melbourne, Australia: Agent Oriented Software \& Codarra Advanced Systems.

Reeve, J. (2005). Understanding motivation and emotion. New York: John Wiley \& Sons.

Rescue Coordination Centre Network (2004). Review of Sweep Width, Retrieved June 3, 2004, from http://www.rcc-net.org/rcc/rddocs

Roche, J.G. (2003). Applying UAV Lessons to Transform the Battlefield, Remarks by the Secretary of the US Air Force at Association of Unmanned Vehicles Systems International, Baltimore, MD, 15 July 2003.

Rosenblatt, F. (1962). Principles of neurodynamics: Perceptrons and the theory of brain mechanisms. Washington, DC: Spartan Books. 
RTCA (1992). DO-178B: Software Considerations in Airborne Systems and Equipment Certification. Washington, DC: RTCA Inc.

Ruff, H.A., Narayanan, S., Draper, M.H. (2002). Human Interaction with Levels of Automation and Decision-Aid Fidelity in Supervisory Control of Multiple Simulated Unmanned Air Vehicles, Presence: Vol. 11, No. 4.

Sander Geophysics Ltd. (2004). Presentation given to Carleton University, Ottawa.

SCPS (1999). Space Communications Protocol Specification - Transport Protocol CCSDS 714.0-B-1: Blue Book. Washington, DC: Consultative Committee for Space Data Systems.

Soza \& Company Ltd. (2004). A brief History of Search Theory and Practice - Appendix C. Retrieved June 3, 2004, from http://www.rcc-net.org/rcc/rddocs

Stergiou, C., \& Siganos, D. (1996). Neural Networks, SURPRISE 96, Vol. 4. London, Great Britain: Imperial College of Science Technology and Medicine.

Stocker, H. (2003). Autonomous Intelligent Systems: Opportunities and Needs for the CF/DND: TM-2003-004, Ottawa, Canada: Defence Research \& Development Canada.

Stockermans, Capt. R. (2004), Search Master - Interview and email correspondences with the author, July-September 2004. JRCC Trenton, Trenton, Canada.

Taylor, R.M, Bonner, M., Dickson, B., Howells, H., Miller, C., Milton, N., et al. (2002). Cognitive Cockpit Engineering: Coupling Functional State Assessment, Task Knowledge Management, and Decision Support for Context-Sensitive Aiding, Chapter 8, Cognitive Systems Engineering in Military Aviation Environments: Avoiding Cogminutia Framentosa, Human Systems Information Analysis Center.

Tesfatsion, L. (2004). Agent-Oriented Programming: Introduction Lecture. Ames, IA: Iowa State University.

Weiss, G. (Ed), (2000). Multiagent Systems: A Modern Approach to Distributed Artificial Intelligence. Boston, MA: The MIT Press.

Winikoff, M., Padgham, L., \& Harland, J. (2001). Simplifying the Development of Intelligent Agents: Technical Document TR-01-03. Melbourne, Australia: RMIT University.

Wong, K.C. (2001). Survey of Regional Developments: Civil Applications, presented at UAV Australia Conference, Melbourne, Australia. 
Wooldridge, M., \& Jennings, N. (1995). Intelligent agents: theory and practice, The Knowledge Engineering Review, Vol. 10, No. 2, pp. 115-152.

Wooldridge, M., \& Jennings, N. (1998). Pitfalls of Agent-Oriented Development. In Proceedings of the $2^{\text {nd }}$ International Conference on Autonomous Agents (Agents '98), Minneapolis, MN.

Wooldridge, M., Jennings, N., \& Kinny, D. (2000). The Gaia methodology for agentoriented analysis and design. Journal of Autonomous Agents and Multi-Agents Systems, Vol. 3, No. 3, pp. 285-312.

Wooldridge, M. (2002). An Introduction to Multiagent Systems. Chichester, Great Britain: John Wiley \& Sons.

Zadeh, L. (1965). Fuzzy Sets. Information and Control, Vol. 8, No. 3, pp.338-353. 


\section{Appendix A: Search and Rescue Practices}

\section{A.1 History of SAR Operations in Canada}

This section provides a brief history of search and rescue and its organization in Canada. Since 1974, the Canadian Forces have been the primary responders for air search and rescue. As the federal agency responsible for the search of an overdue or missing aircraft, the Department of National Defence (DND) Canadian Forces (CF) carry out the missions, often with the assistance of the Civil Air Search and Rescue Association (CASARA). As a national association that was formed in 1986, CASARA is composed of local volunteer groups. CASARA members supply their own light aircraft, time and skills to provide trained crews of pilots, navigators, and spotters. With additional aircraft and local knowledge, CASARA crews supplement the CF. With SAR squadrons based across the country the CF also works closely with the Canada Coast Guard (CCG), providing air assets for all marine incidents in waters under federal jurisdiction, while the Canada Coast Guard and Canadian Coast Guard Auxiliary provide primary marine resources. Together the CF and CCG run the three Joint Rescue Coordination Centres (JRCCs) in Halifax, Nova Scotia, Trenton, Ontario, and Comox, British Columbia, that coordinate and control maritime and aeronautical searches (National Search and Rescue Secretariat-NSS, 2004).

Other members of the Canadian SAR community include Parks Canada which provides search and rescue services within national parks, national marine conservation areas and reserves, national historic sites and historic canals. The Royal Canadian Mounted Police responds to SAR incidents outside of national parks and in various municipalities, the 
territories, and all provinces except Ontario and Quebec. Meteorological Service of Canada provides information on climate, weather, ice and other environmental data of particular interest to the SAR community (NSS, 2004).

For any SAR operation there is a search coordinator who is responsible for the overall command, control and communication. The coordinator's search planning sequence involves five steps:

1. Estimate the datum or position of the emergency, taking into account wind speeds and directions and/or current drift.

2. Determine the size of the search area, allowing for errors in position and navigation by the search units.

3. Select the search pattern by considering the terrain and size of the area.

4. Determine the area coverage and identify the probability of detection based on the terrain, track spacing and number of sweeps.

5. Develop an optimum search plan considering the search aircraft's capabilities, weather, terrain and any other limiting factors.

\section{A.2 Estimating the Datum}

In the case of a missing aircraft, the position of the emergency is the region centred at the last known position (LKP) of the aircraft. The region extends to the limit of the vehicle's endurance in all possible directions. Based on the empirical data collected from actual SAR incidents during the period of 1981-1986, it was found that the most probable area 
for many missing aircraft exists along the intended track between the LKP and the destination.

\section{A.3 Determining Search Areas}

The Canadian Search Area Definition (CSAD) was developed for plotting inland searches in Canada when the LKP is available. Figure A-1 is found in Chapter 6 of the NSM (1998) showing the initial search area contained within 10 nautical miles of the intended route, which is later expanded to 15 nautical miles forward of the LKP if nothing is found following the CSAD 1, pass. When the pilot's intended route is not known with any certainty, then all likely routes must be covered.
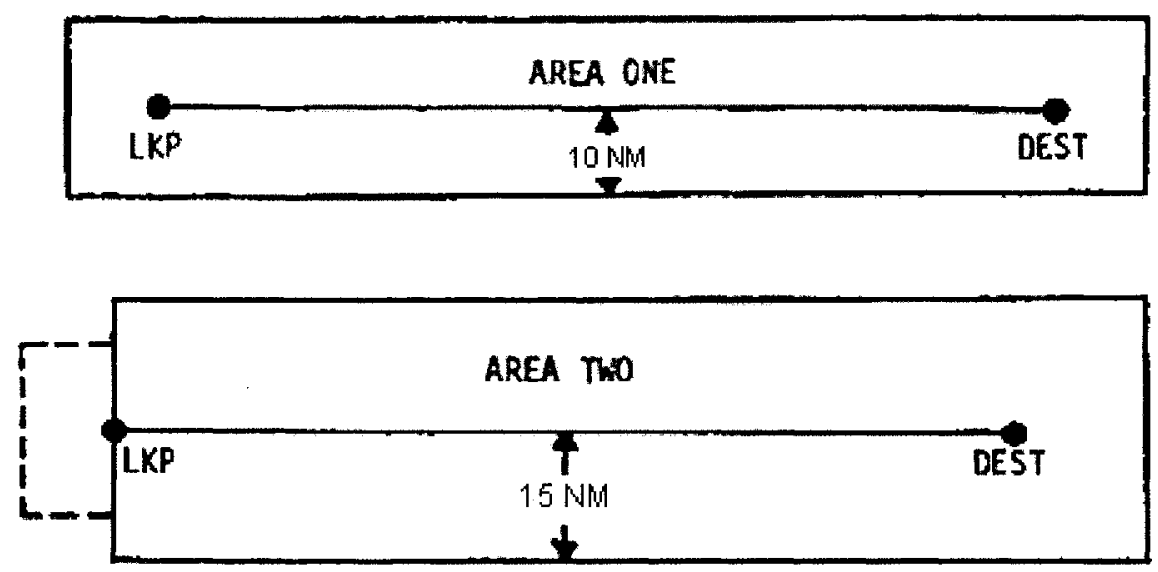

Figure A-1: CSAD $1 \&$ CSAD 2 area definitions (NSM, 1998)

\section{A.4 Selecting the Search Patterns}

There are six search patterns used for SAR operations. Each pattern is described, along with a description of its application. The patterns begin at a common starting point (CSP) 
and use a track spacing of $(\mathrm{S})$. All figures in this section were obtained from Chapter 5 of the NSM (1998).

The track crawl, shown in Figure A-2, is used when it is assumed that the search object will be close to its intended track. The pattern is used on both electronic and visual searches.

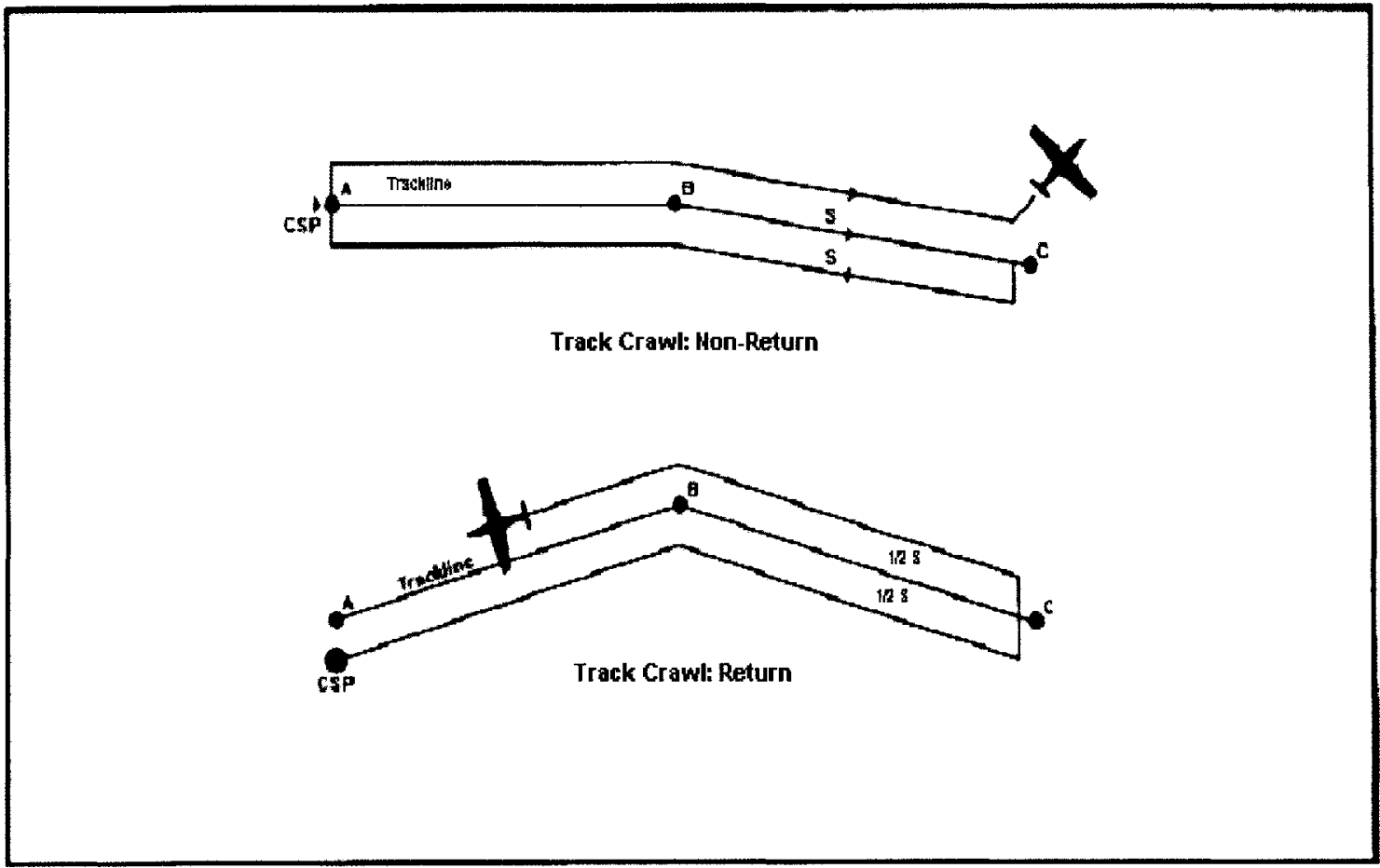

Figure A-2: Track crawl patterns (NSM, 1998)

The creeping line (Figure A-3) is used when only an approximate position of the target is known. This search pattern provides uniform coverage and enables the search vehicle to rapidly advance along the given track line. With the creeping line search, the lines are parallel to the shortest side of the search area. 


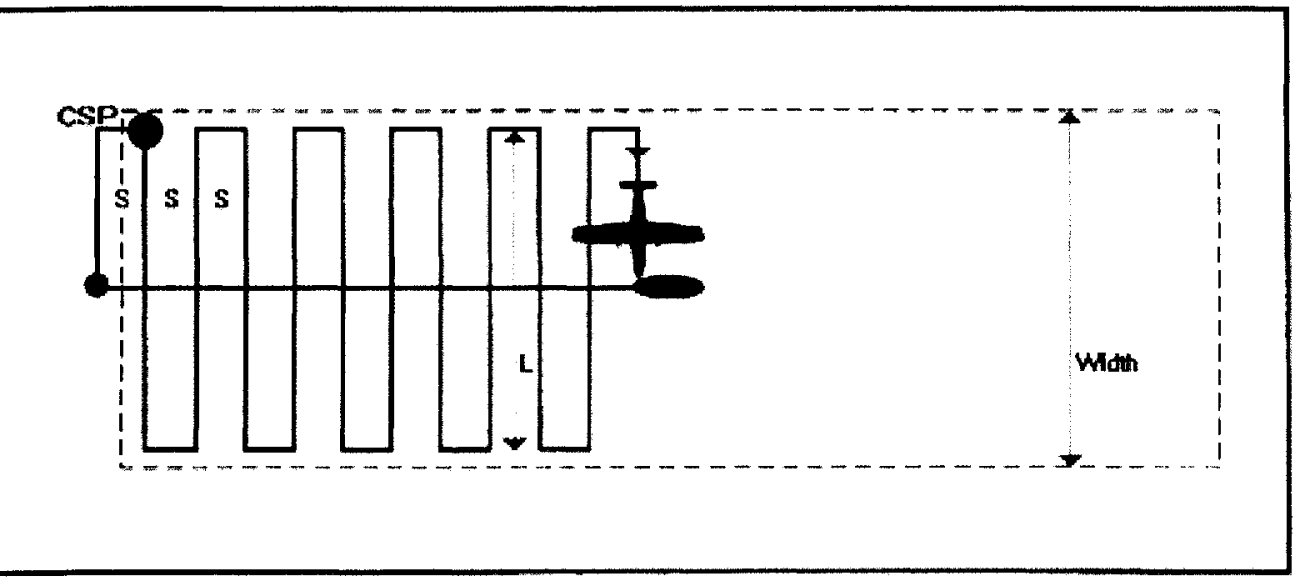

Figure A-3: Creeping line pattern (NSM, 1998)

The parallel track shown in Figure A-4 is similar to creeping line except that the lines are parallel to the longest side of the search pattern. Again a sweeping pattern is followed. While not illustrated in the figures, SAR pilots indicate that it is common practice to perform the turns beyond the border of the search width, and re-enter the search region perpendicular to the boundary (J. Bernier, personal communication, 1 Sept 2004).

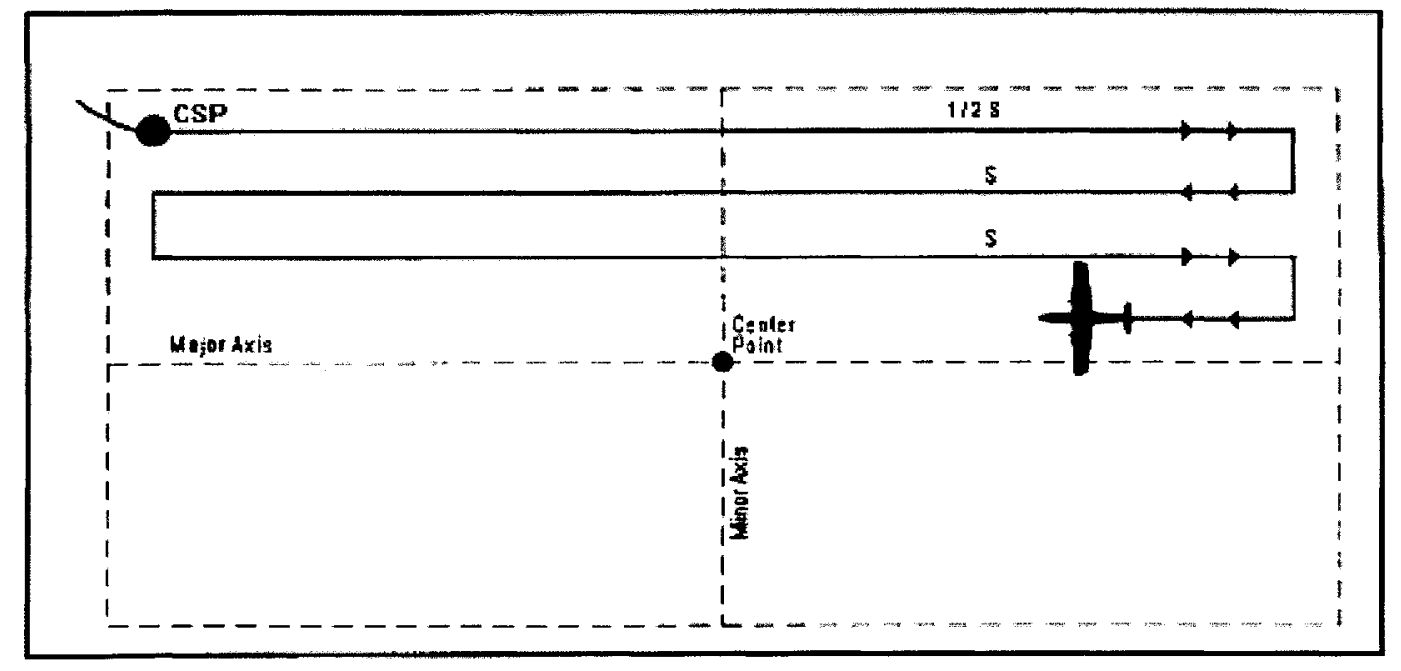

Figure A-4: Single unit parallel track (NSM, 1998)

The expanding square (Figure A-5) is used when the location of the target is known within 100 square miles. In order for this pattern to be successful, the pattern requires 
precise navigation to avoid gaps. If a second pass is required, it is oriented $45^{\circ}$ from the initial pass.

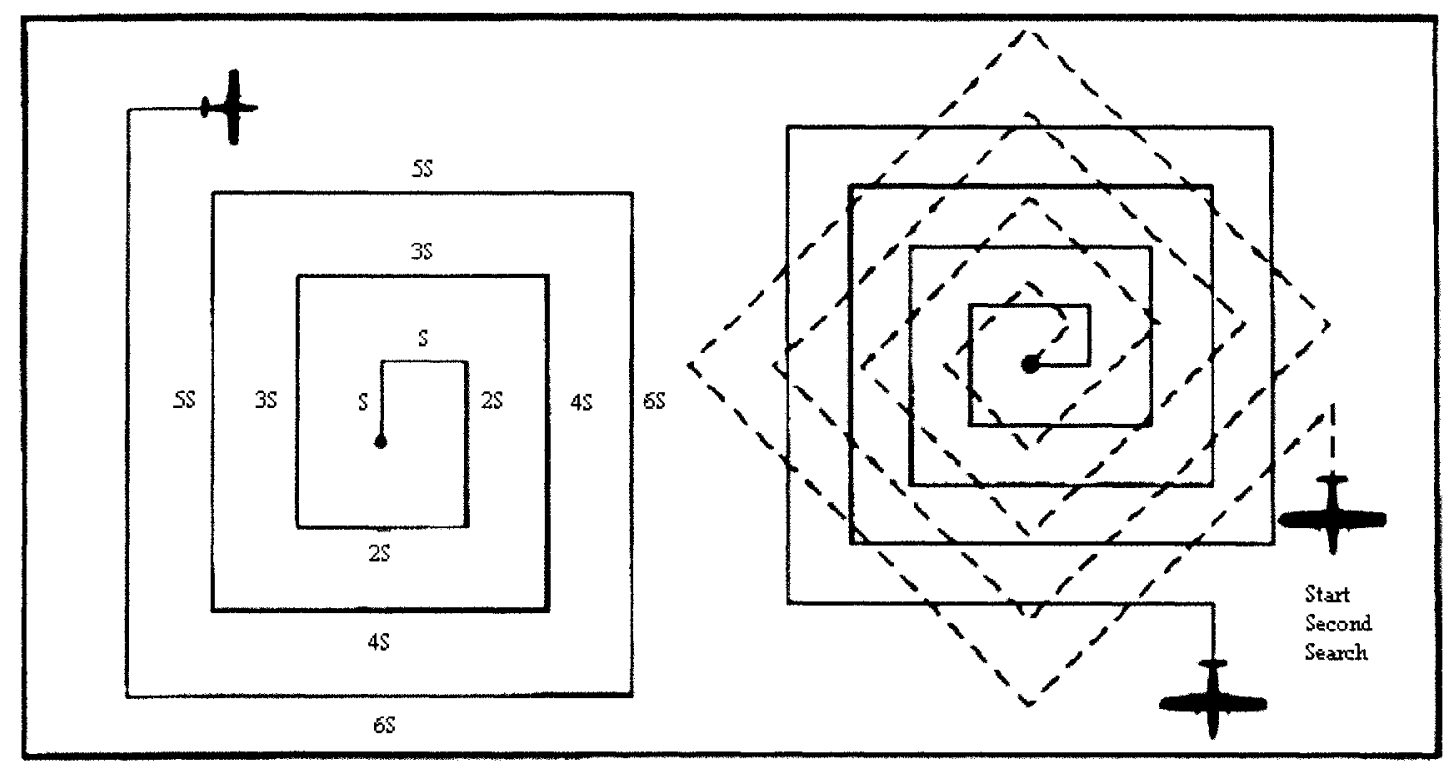

Figure A-5: Expanding square (NSM, 1998)

Sector patterns, shown in Figure A-6, are used when the datum is established with a high degree of confidence, the search area is not extensive, and the target is difficult to detect. This pattern results in very small track spacing at the centre and therefore a higher probability of detection. Usually a six-sector pattern is used, with each sector having a radius less than 10 nautical miles. In this pattern, each turn is $120^{\circ}$ to the right. If a second search is required, then it is offset by $30^{\circ}$ from the first. 


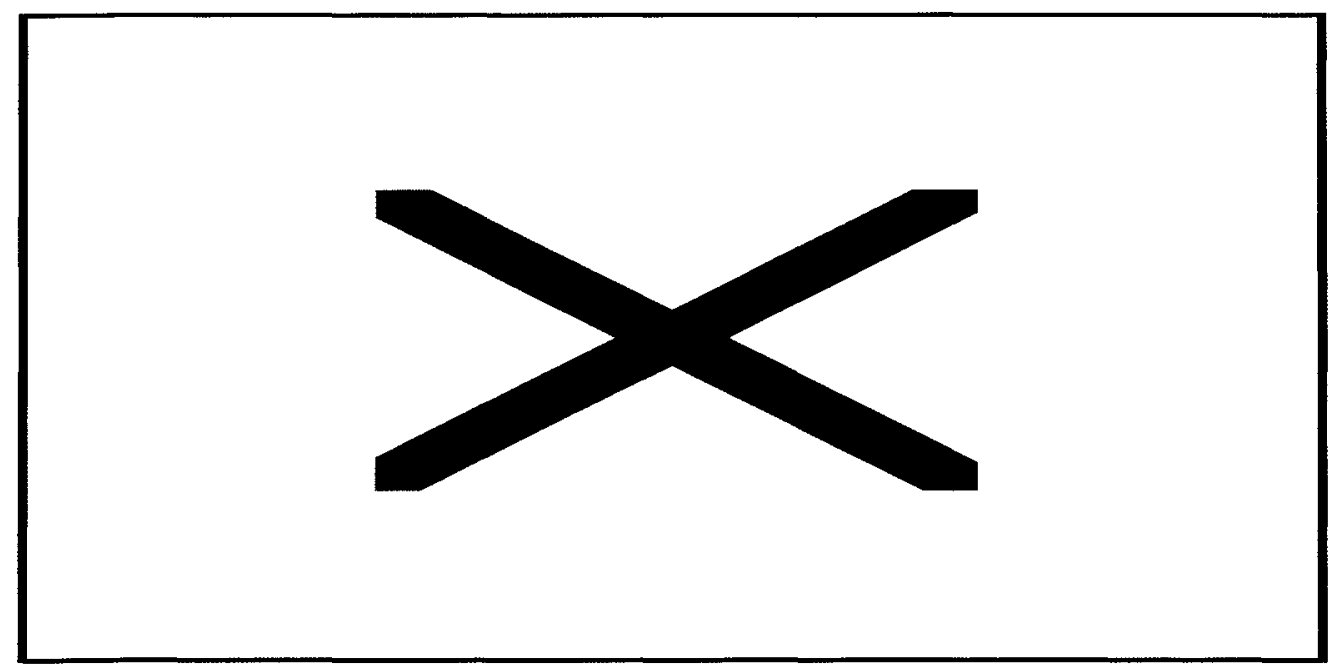

Figure A-6: Sector search (NSM, 1998)

A contour search (Figure A-7) is a hazardous search procedure and is only used when the search aircraft are highly maneuverable, with small turning radius and adequate reserve power. The search crews must be well briefed about flying in mountainous terrain.

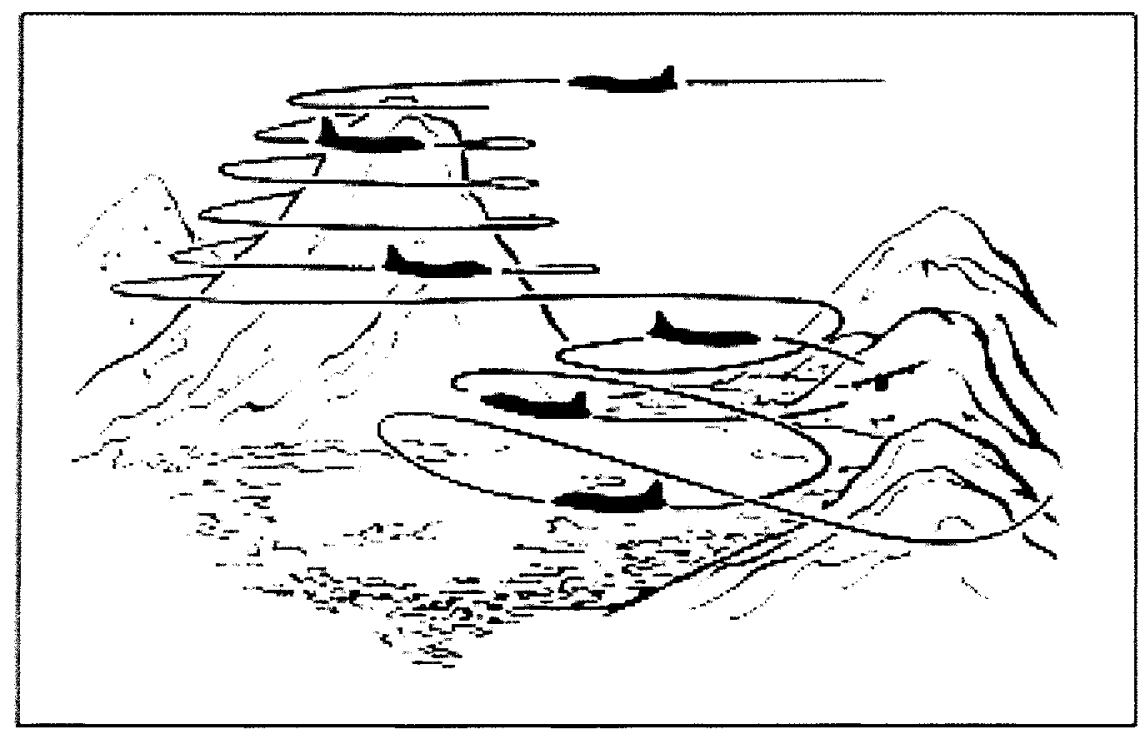

Figure A-7: Contour search (NSM, 1998) 
Until UAV systems are able to closely follow terrain and avoid obstacles, the contour search is beyond the scope of their capabilities. However, due to the dangers involved in this kind of search, it is an ideal candidate for future UAV systems.

\section{A.5 Determining Area Coverage}

When the incident is known to have occurred in the vicinity of a position or datum, the probability of containment (POC) is defined as follows:

$$
P O C=1-\exp \left(\frac{-R^{2}}{2}\right)
$$

Often the POC is estimated and then the value of the circle's radius (R) is determined. The probability of detection (POD) is a sensor's ability to detect a particular type of search object under a given set of operational and environmental conditions. The POD is a function of the type of the terrain, the effort, the area covered and the lateral range curve. It is an average measure of detection over an area. Multiple factors influence detection capability; however, they can be reduced to four expressions:

\section{Track Spacing (S)}

This is the distance between adjacent search tracks. With smaller spacing (S) there is a higher likelihood of detecting the target, however this also increases the time it takes to search an area. The major constraints in any search are time and resources available. 
Probability of Detection (POD)

Expressed as a percentage these are the odds of seeing the target. This is highly dependant on the sensor being used. It is easy for the human eye to see targets that are close to the patrol aircraft but it is impossible at great distances. Koopman (1980) established basic search theory during World War II with an aim of providing an early detection of enemy forces and in particular naval targets. Only later after the war was this work applied to search and rescue.

Sweep Width (W) and Coverage Factor (C)

Track spacing (S) and sweep width (W) control the coverage factor(C) are illustrated in Figure A-8. Where the coverage factor is a measure of effectiveness, sweep width is always less than the maximum coverage range, and depends on whether a visual or electronic search is being conducted. 


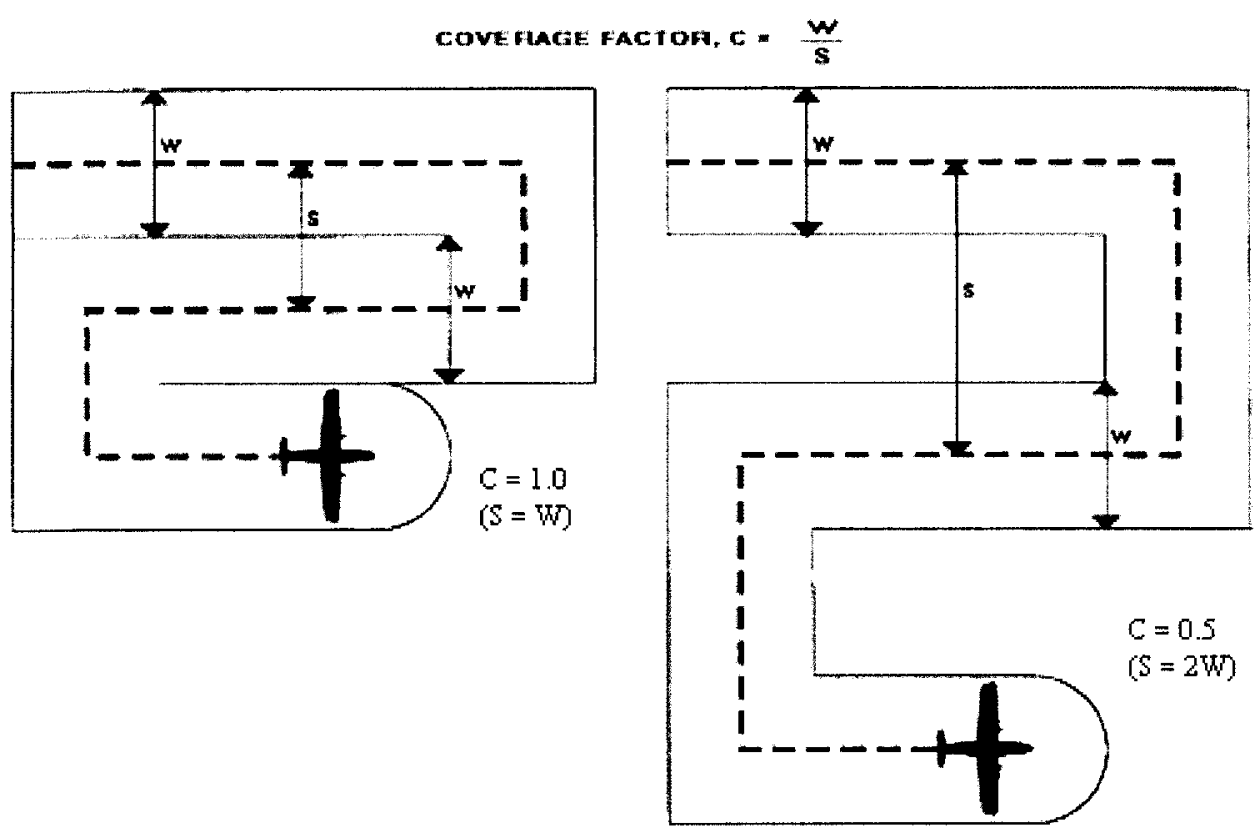

Figure A-8: Coverage factor (NSM, 1998)

According to Soza \& Company (2004), Koopman made two key contributions to today's Search and Rescue methods: the inverse cube law for visual detection, and the development of the lateral range curve (LRC). Both contributions are used today in calculating the probability of detection.

Koopman's inverse cube model makes the following assumptions about the target

- The target is located randomly within the search area and remains in the area for the entire search.

- The area is covered by equally-spaced parallel search tracks.

- The environmental conditions remain constant throughout the search.

- A constant search speed is maintained. 
- A number of passes are made on both sides of the target by the searcher.

- The instantaneous probability of detecting the target is dependent on the surface area and the intrinsic contrast of the target.

- The altitude of the search is small compared with the range of the target.

The LRC describes the cumulative probability of detecting a target during one complete transit of an infinitely long straight track as a function of the perpendicular distance from the track to the target. The inverse cube law produces a highly bell-shaped LRC where the POD is greater than $80 \%$ for targets along the track line or at very short distances either side, and then falls rapidly toward zero. These curves change under different weather conditions as shown in Figure A-9, adopted from Champagne, Carl and Hill (2003).

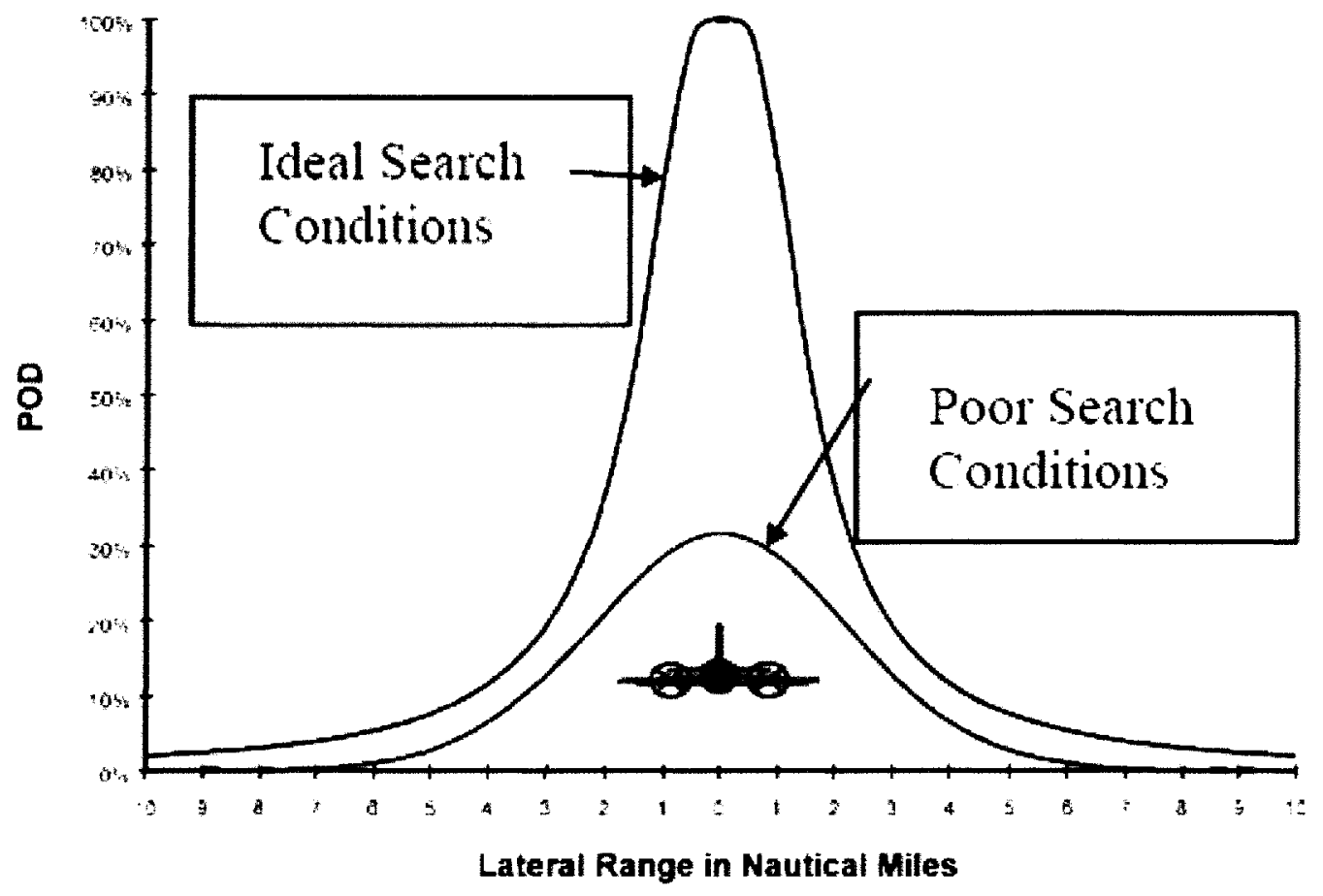

Figure A-9: POD of the visual system under different search conditions 
The probability of detection is a sensor's ability to detect a particular type of search object under a given set of operational and environmental conditions. The POD is a function of the type of search pattern used, the effort, the area covered and the lateral range curve. It is an average measure of detection over an area. For parallel path searches for an area of size (A) using track length (z) and perfect tracking with spacing, the POD for the inverse cube model is given by Abi-Zeid and Lamontagne (2003)

$\operatorname{POD}(z)=\operatorname{erf}\left(\sqrt{\pi} \frac{W z}{2 A}\right)$ for $z \geq 0$

The detection model will depend on the lateral range function of the sensor $[\mathrm{p}(\mathrm{x})]$, the sweep width (W), the total effort available $(\mathrm{z})$, the probability of detection $[\mathrm{POD}(\mathrm{x})]$ and the coverage.

For a visual model the range function in a line search is as follows.

$$
p(x)=1-\exp \left(\frac{-2 k h}{v x^{2}}\right) \text { for } x>0
$$

Where: $\mathbf{x}=$ lateral range to the target (perpendicular distance from sensor track line); $\mathbf{h}=$ altitude; $\mathbf{v}=$ speed; $\mathbf{k}=$ a constant that depends on the area of the target, the visibility, and other fixed search factors. 
The probability of success in finding a target will depend on the environment and search pattern used, the vehicle's speed, coverage factor and the capabilities of the onboard sensors. 


\section{Appendix B: Decision Making}

\section{B.1 Artificial Neural Networks}

By design, artificial neural networks process information in a similar fashion to the human brain. The network contains a large number of interconnected processing nodes (neurons), which are organized into different layers. The network operates by creating connections between many different processing nodes, each analogous to a single neuron in a human brain. Each node takes many input signals, then, based on an internal weighting system, produces a single output signal that is sent to another node. One of the basic blocks in artificial neural networks is the McCulloch-Pitts model for a neuron, shown in Figure B-1. The model receives one or more inputs and produces one or more identical outputs, each of which is a simple non-linear function of the sum of the inputs to the neuron. The influence that each input has on the decision making is dependent on the weight of the particular input. The weight of an input is a number which, when multiplied with the input, gives the weighted input. Weighted inputs are then added together and if they exceed a pre-set threshold value, the neuron produces an output. If the threshold is not met, the neuron does not produce an output.

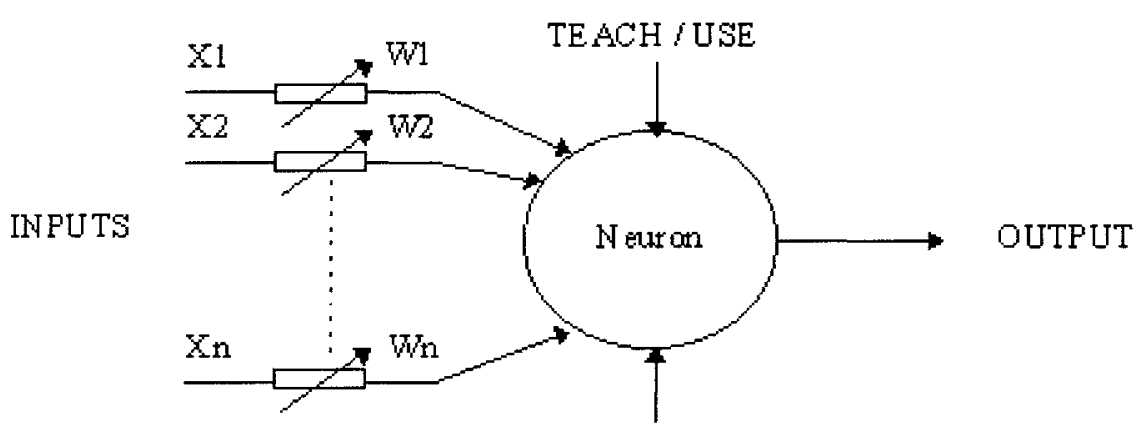

TEACHING INPITT

Figure B-1: McCulloch-Pitts neuron model 
Neural networks learn by example, and cannot be pre-programmed to perform a specific task. There are two ways to teach a neural network, either through supervised or unsupervised learning.

Supervised learning uses an external teacher so that each output unit is told what its desired response to input signals ought to be. In the case of controlling a plant, the neural network would be setup in parallel to the existing controller, as shown in Figure B-2.

Supervised learning includes error-correction learning, reinforcement learning and stochastic learning. An important issue concerning supervised learning is the problem of error convergence, that is, the minimization of error between the desired and computed unit values. The aim is to determine a set of weights $\left(W_{1} \ldots W_{n}\right)$ in order to minimize this error.

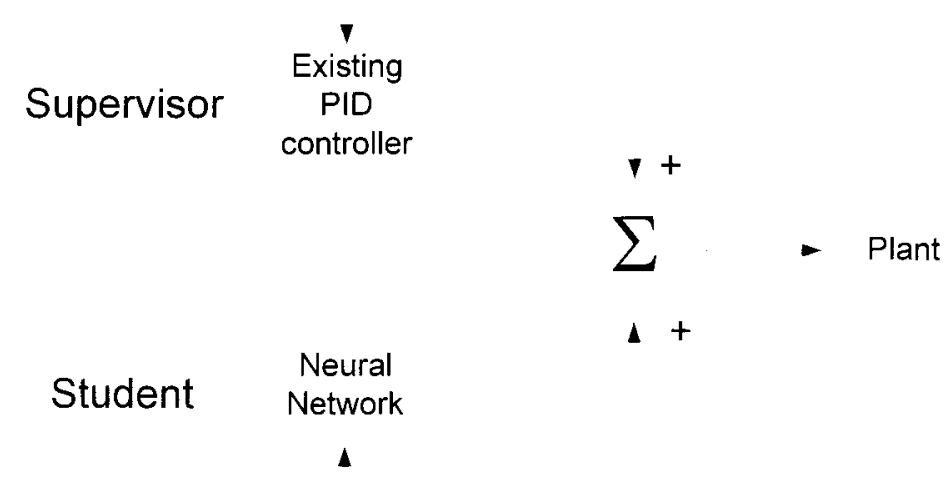

Figure B-2: Online, parallel process training 
Unsupervised learning uses no external teacher and is based upon only local information. It is also referred to as self-organization, in the sense that it self-organizes data presented to a network and detects their collective properties (Sterigou \& Siganos, 1996).

\section{B.2 Fuzzy Logic}

Fuzzy logic and Boolean logic are both based on facts; however, fuzzy logic extends Boolean logic to handle vague concepts. To express vagueness in a quantitative fashion, fuzzy logic introduces a membership function that maps elements to real values between zero and one (inclusive). The value indicates the "degree" to which an element belongs to a set. A membership value of zero indicates that the element is entirely outside the set, whereas a membership value of one indicates that the element lies entirely inside a given set. Any value between the two extremes indicates a degree of partial membership to the set. Although fuzzy logic has the ability to deal with imprecise notions, it is still a purely objective form of logic; membership in a fuzzy set is determined strictly on the basis of raw data about a given entity. In other words, fuzzy logic does not include the notions of "belief", "plausibility", "doubt", or "conflicts of evidence" (Brule, 1985).

To better grasp the concepts of a fuzzy system, a classical example considers a system that is designed to mimic the diagnostic powers of a physician. One of the major tasks is to codify the physician's decision-making process. The designer soon learns that the physician's view of the world, despite a dependence upon precise scientific tests and measurements, incorporates evaluations of symptoms, and the relationships between them, in a "fuzzy," intuitive manner before any decision is made regarding what or how 
much of a particular medication to administer. A decision will have had as much to do with the physician's sense of the relative "strength" of the patient's symptoms as it will to do their height/weight ratio. While some of the decisions and calculations could be done using traditional logic, fuzzy logic systems afford a broader, richer field of data and the manipulation of that data.

\section{B.3 Human Decision Making}

While understanding how humans make decisions is a study unto itself in social and cognitive psychology, the decision making engine within an intelligent agent subscribes to a similar decision making process that humans follow.

The early works of Miller, Galanter and Pribram (1960) investigated how plans motivate behaviour. According to these pioneers, people have mental representations of the ideal states of their behaviour, environmental objects, and events. People are also aware of the present state of their behaviour, environment, and events. Any mismatch between one's actual and ideal state is perceived as an incongruity as shown by the Test-Operate-TestExit model shown in Figure B-3. 


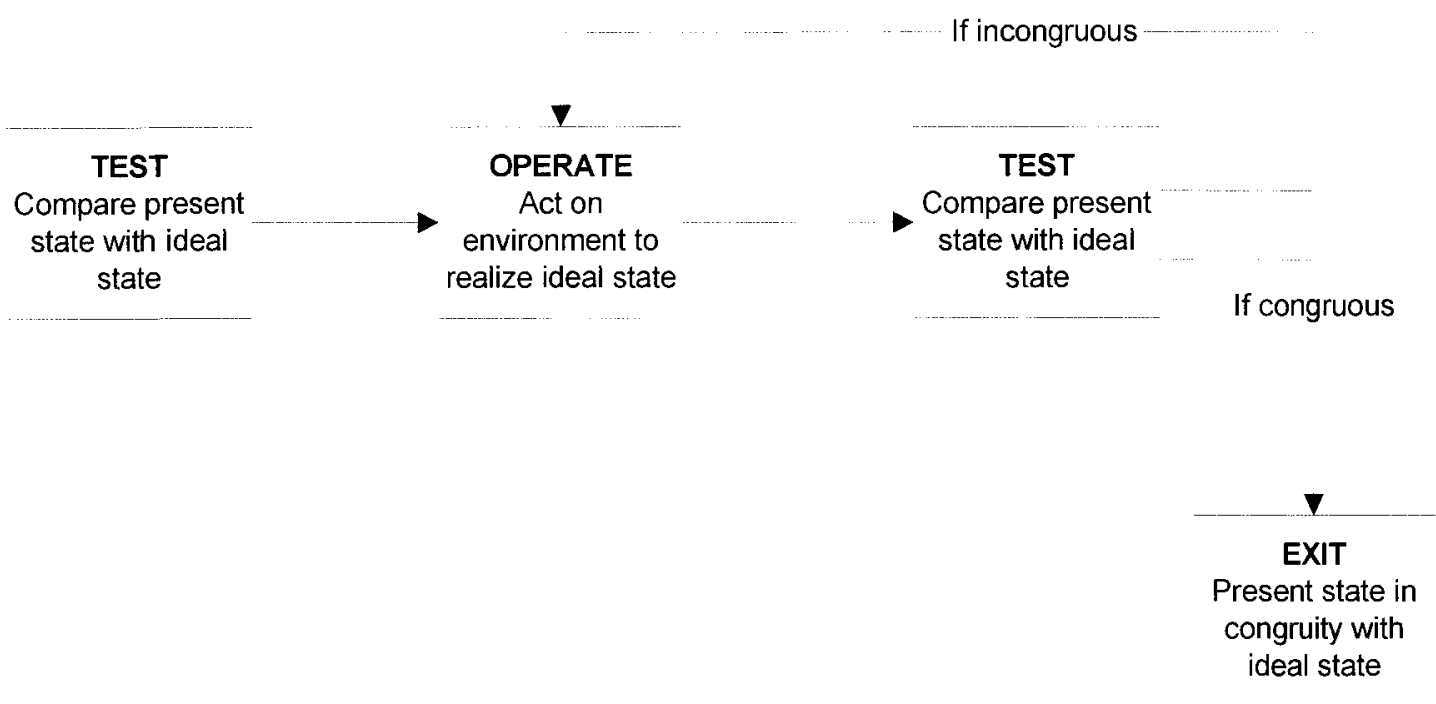

Figure B-3: Schematic of the test-operate-text-exit (TOTE) model

An incongruity acts as the motivation for action, instigating plans in order to reach an ideal state. The plan for this corrective motivation portrays individuals as (1) detecting present-ideal inconsistencies, (2) generating a plan to eliminate the incongruity, (3) instigating plan-regulated behaviour, and (4) monitoring feedback as to the extent of any remaining present-ideal incongruity (Reeve, 2005). Plans are modifiable because human beings are active decision makers and choose which process to follow. If the original plan is ineffective, it is changed or revised, in order to achieve the ideal state.

Two types of discrepancy processes exist: discrepancy reduction and discrepancy creation (Bandura, 1990; Carver \& Scheier, 1998). The first is consistent with a feedback loop of the TOTE model of human decision making, where the error between ideal and actual is reduced, while the latter is consistent with a feed-forward loop. With discrepancy creation, higher goals are proactively set, and this loop does not require feedback. Still, goals do not always enhance performance; only those goals that are 
difficult and specific do so (Locke et al. 1981, Locke 1968). Difficult goals energize human subjects to focus their attention and efforts. With the use of timely feedback subjects are able to keep track progress towards meeting these goals. However, feedback only provides information and a standard of performance by which to measure it is still required. As such, the feedback itself requires a goal. When goals are attained from this process the result is an emotion of either satisfaction or dissatisfaction.

Intentions are another mechanism used in the pursuit of goal attainment. This closeminded approach allows us to avoid distractions, and focus on the tasks at hand. Coupling goals with intentions is an important step that ensures that tasks are actually carried out. 


\section{Appendix C: Map Systems}

Due to the curvature of the earth, the length of one degree changes depending on where you are in the world. The Lambert azimuthal Equal-Area Projection was developed by Lambert in 1772 and is typically used for mapping large regions like continents and hemispheres. An azimuthal, equal area projection, exhibits zero distortion at the center of the projection, and the distortion increases radially away from this central point. As shown in Figure C-1, for each degree of latitude, there is a noticeable difference in distance along each parallel, while the distances along the meridians remain relatively constant.
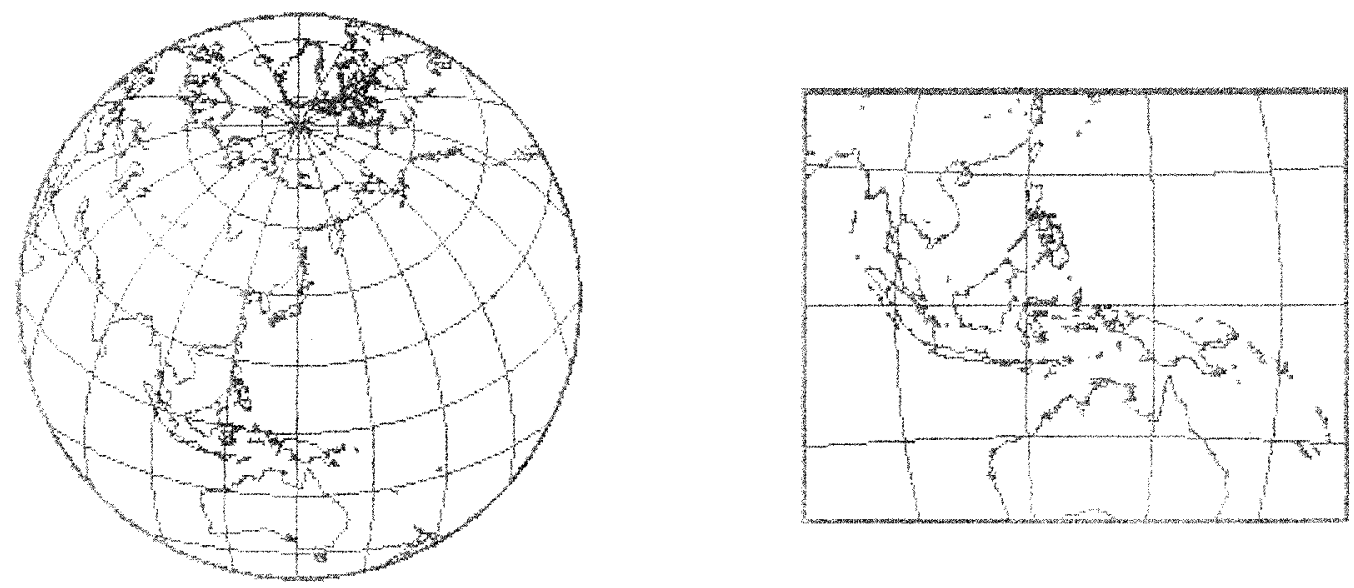

Figure C-1: Lambert azimuthal projection on the Earth

For this reason, it is not possible to simply convert any particular latitude and longitude to metres. However, using geodetic information obtained from Amiran \& Schick (1961), it is possible to plot the changes in length for one degree of latitude for both the meridians (longitudes - Figure C-2) and parallels (latitudes - Figure C-3). Using $3^{\text {rd }}$ order curves to approximate the data, all data points lie on the curves and are accounted for by these equations, as stated on their respective plots. 
Length of One Degree on Meridian in Meters Centred on Every Degree of Latitude

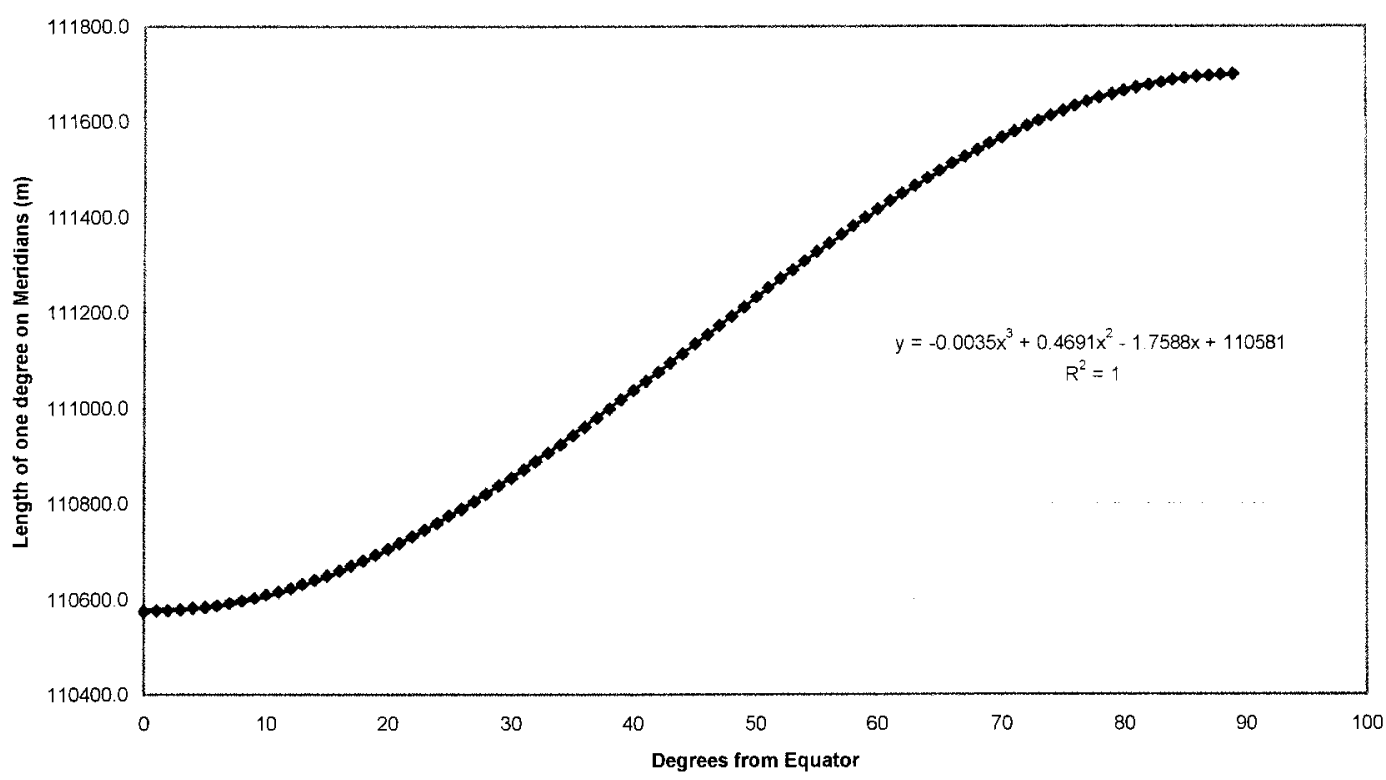

Figure C-2: Length of degree on meridians

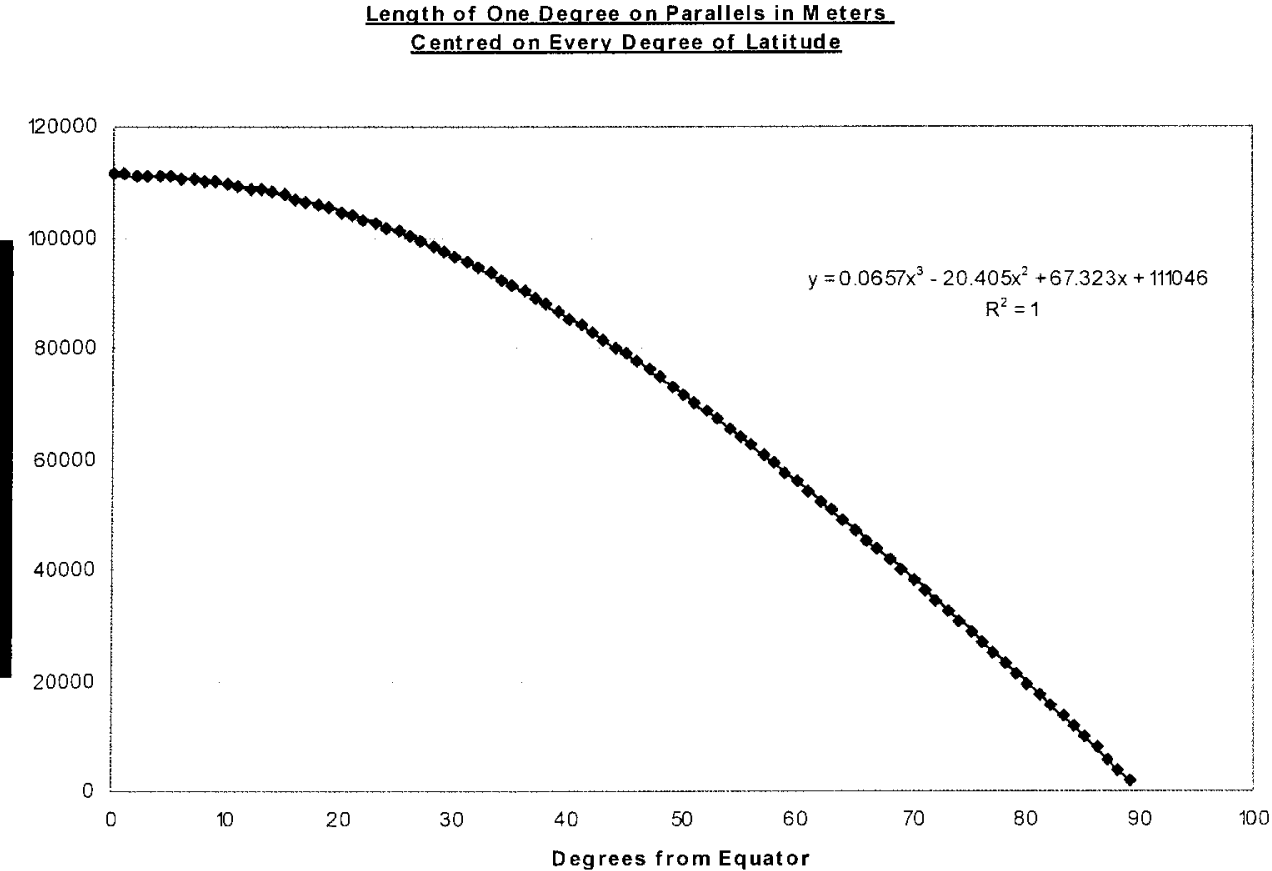

Figure C-3: Length of degree on parallels 
Thus, there is a need for look-up tables during the mission-planning phase to establish what values should be used for conversion from degrees to metres. As shown by the tables, the length of the meridians remains almost constant; however, the length along the parallels differs significantly. To add complexity to this issue, the precision within the MicroPilot 2028 autopilot hardware has a maximum resolution of $\mathbf{0 . 0 0 0 1}$ degrees.

Table C-1: Conversions Tables from Degrees to Metres

(a)

(b)

At 40 degrees from the Equator

\begin{tabular}{|l|l|l|}
\hline Length of: & Meridian (m) & Parallel $(\mathrm{m})$ \\
1 & 111037.208 & 85295.72 \\
0.1 & 11103.7208 & 8529.572 \\
0.01 & 1110.37208 & 852.9572 \\
0.001 & 111.037208 & 85.29572 \\
$\mathbf{0 . 0 0 0 1}$ & $\mathbf{1 1 . 1 0 3 7 2 0 8}$ & $\mathbf{8 . 5 2 9 5 7 2}$ \\
0.00001 & $\mathbf{1 . 1 1 0 3 7 2 0 8}$ & 0.8529572 \\
\hline
\end{tabular}

At 75 degrees from the Equator

\begin{tabular}{|l|l|l|}
\hline Length of: & Meridian (m) & Parallel (m) \\
1 & 111611.215 & 29034.2875 \\
0.1 & 11161.1215 & 2903.42875 \\
0.01 & 1116.11215 & 290.342875 \\
0.001 & 111.611215 & 29.0342875 \\
$\mathbf{0 . 0 0 0 1}$ & $\mathbf{1 1 . 1 6 1 1 2 1 5}$ & $\mathbf{2 . 9 0 3 4 2 8 7 5}$ \\
0.00001 & 1.11611215 & 0.290342875 \\
\hline
\end{tabular}

Because the closer look algorithm uses waypoints relative to the location where the target was spotted $\left(\mathrm{O}_{x}, \mathrm{O}_{y}, \mathrm{O}_{z}\right)$, the implications of global positioning is clear. For example, to complete the $180^{\circ}$ turn, the vehicle is commanded to $\left(\mathrm{O}_{\mathrm{x}}+100 \mathrm{~m}, \mathrm{O}_{\mathrm{y}}+224 \mathrm{~m}, \mathrm{O}_{z}\right)$.

While flying at 40 degrees from the Equator and using lat/lon in degrees:

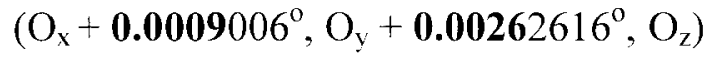

While flying at 75 degrees from the Equator and using lat/lon in degrees: 


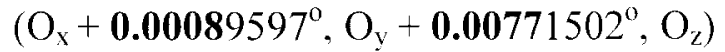

Therefore, prior to any UAV flights, an appropriate database must be pre-selected, as the values will influence the selection of an appropriate conversion factor. 